\title{
Search For Gluino Pair Production Using Like-Sign Top Dilepton Events
}

\author{
Chadd Smith \\ A dissertation submitted to the Johns Hopkins University in conformity with the \\ requirements for the degree of Doctor of Philosophy. \\ Baltimore, Maryland \\ 2002
}

Copyright (c) 2002 by Chadd Smith, All rights reserved. 


\begin{abstract}
We present the results of a search for supersymmetry via gluino pair production in $p \bar{p}$ collisions at $\sqrt{s}=1.8 \mathrm{TeV}$. The data sample represents the full $106.1 \mathrm{pb}^{-1}$ of integrated luminosity collected by the Collider Detector at Fermilab (CDF) during Run I of the Tevatron. The gluino, a supersymmetric partner of the gluon, is expected to be strongly produced at the Tevatron. The scalar top, superpartner of the top quark, is believed to be the lightest squark and the only squark less massive than the gluino. In the region of parameter spaced defined by $\left(m_{t}+m_{\tilde{t}}\right)<m_{\tilde{g}}<\left(m_{q}+m_{\tilde{q}}\right)$, $(\tilde{g} \longrightarrow t \tilde{t})$ is the preferred decay channel. The Majorana nature of the gluino gives rise to like-sign top quarks from $\tilde{g} \tilde{g}$ events. We use the top dilepton analysis to search for this unique signature in the like-sign dilepton channel, and we set upper limits on the cross section for gluino-gluino production. Despite a low expected background contribution of $0.67{ }_{-0.24}^{+1.54}$ events, we observe two like-sign $e \mu$ events and one trilepton event. This experimental result, and the difficulty of probing gluino masses close to the top mass due to the systematics surrounding a light stop, prevents the establishment of a mass limit on the gluino. Whether the observed excess in the data is simply a statistical fluctuation or a hint of "new physics" will be a subject of interest at the Tevatron during Run II.
\end{abstract}




\section{Acknowledgements}

Just as today's modern particle physics experiments require the efforts of hundreds (and now thousands!) of collaborators, this thesis would not have been possible without the countless people who have influenced and encouraged me throughout the years. I have been truly fortunate to have shared my life and work with so many wonderful friends and family. To single out each and every one of you would require more pages than all the numbers and figures that follow, so please don't be alarmed if I'm unable to mention you by name. I hope you know that each of you holds a special place in my heart.

Thanks to Bill Hooper and Van Daniel at Clinch Valley College and Chris Allgyer and Bill Harris at Mountain Empire Community College. Your commitment to bringing science to local high school students is highly admirable, and you built wonderful programs for the two colleges in southwestern Virginia. I wish I could have been a part of the local community of science educators with you. Dr. Hooper and Dr. Daniel, I hope you know how much my parents thought of you when you taught them and how much you influenced me at a young age. Dr. Allgyer and Dr. Harris, thank you for giving me an opportunity to work as a teaching assistant and pass along my love of science to the younger students who came along years after me.

During my years at Virginia Tech, I was lucky enough to part of two families. In the Mathematics department, Wayne Patty, J.U. Kim, and John Shaw were all terribly kind to me. I wish I had spent more time on your side of campus. Sorry for not becoming a mathematician, but I could not have come this far as a physicist without all the skills you taught me.

On the Physics side, Sherri Turner and C.D. Williams offered plenty of support and guidance to me early on. John Simonetti and Brian Dennison in the astronomy group allowed me to study a subject I have loved ever since second grade, and I hold

many fond memories of my involvement in the Astronomy Club. But it was Leo Piilonen and $\mathrm{Al}$ Abashian who nurtured my interest in particle physics and provided my first opportunity to work in the field. Those two summers working on the MEGA experiment at the Meson Physics Facility in Los Alamos were easily the best of my 
life. Thanks to Martin Cooper and Richard Mischke at LAMPF; Leo and Dan Haim from Virginia Tech; Don Koetke, Bob Manweiler, and Shirvel Stanislaus at Valparaiso University; and everyone else on the project. I'm so happy to see that all your years of hard work eventually paid off with the final published result. I would also like to thank Sam McKenzie and Ed Arakawa for opening up their lab to me for a summer at Oak Ridge National Laboratory.

At Johns Hopkins, I've had the privilege of working and studying under many of the finest professors around, and I greatly miss being part of the family there. Special thanks go out to Barry Blumenfeld, David Gerdes, C.Y. Chien, Morris Swartz, and everyone on the 6th floor. Leon Madansky and Arthur Davidson, you are greatly missed.

Above all, I owe the greatest debt of gratitude to Bruce Barnett. Looking back, there are many aspects of this analysis and my graduate school experience that I wish I could go back and change, but the one thing I would keep constant is the choice of you as my advisor. Your wisdom and infinite patience really made this possible. I hope you are proud of me.

Thanks to Regina Demina for convincing me to pursue this analysis and for aiding me early on. Thanks to Mark Kruse for sharing his experience and for taking the time to revisit his analysis to help guide me along. Thanks to Steve Worm, Stephan Lammel, Ray Culbertson, and Max Chertok in the Exotics group for all of their help and interest in the analysis. Finally, thanks to Henry Frisch at the University of Chicago for so graciously giving me a home away from Fermilab and for all of his guidance.

Last, but certainly not least, I would like to thank my family and my wonderful wife, Pai. 


\section{Contents}

List of Figures $\quad$ vi

List of Tables $\quad$ vii

1 Introduction $\quad 1$

2 Theoretical motivation 4

2.1 What is everything made of? . . . . . . . . . . . 4

2.1.1 Atoms and their constituents ............ . 4

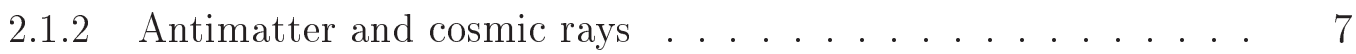

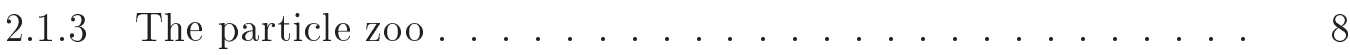

2.1.4 The quark model . . . . . . . . . . . . . . 10

2.1.5 The final pieces of the puzzle ............ 11

2.2 The Standard Model . . . . . . . . . . . . . . . . . . . . . . 13

2.2.1 Particles and spin .................. 13

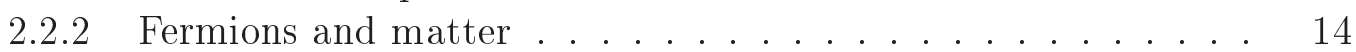

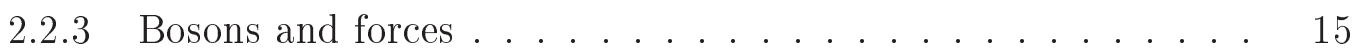

2.3 What's wrong with the SM . . . . . . . . . . . . . 17

2.4 The Higgs particle . . . . . . . . . . . . . . . . . . . 19

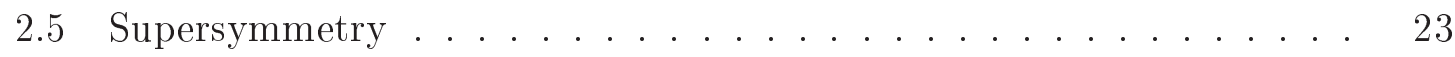

2.6 Gluino production and decay . . . . . . . . . . . . . . . 27

2.7 Top decay . . . . . . . . . . . . . . . . . . . . . . . . 29

2.8 Search signature for SUSY . . . . . . . . . . . . . 31

3 The CDF Experiment $\quad 34$

3.1 The Tevatron ........................ 34

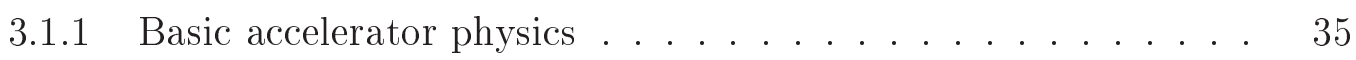

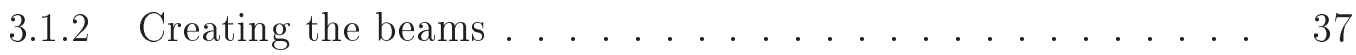

3.2 Collider Detector at Fermilab (CDF) . . . . . . . . . . 39

3.2.1 Some definitions . . . . . . . . . . . . . . . . . 39

3.2.2 Detector overview . . . . . . . . . . . . . . . . . . . . 42

3.3 Silicon Vertex Detector $(\mathrm{SVX}) \ldots \ldots . \ldots . \ldots . \ldots 43$ 
3.4 Vertex Time Projection Chamber (VTX) . . . . . . . . . . 45

3.5 Central Tracking Chamber (CTC) . . . . . . . . . . . 46

3.6 The CDF Calorimeters . . . . . . . . . . . . . . . . 49

3.6.1 Central electromagnetic calorimeter $(\mathrm{CEM}) \ldots . . . . .50$

3.6.2 Central hadronic calorimeters (CHA,WHA) . . . . . . . . 53

3.7 Muon detectors . . . . . . . . . . . . . . . . 54

3.8 Event triggers . . . . . . . . . . . . . . 56

4 Event Selection $\quad 61$

4.1 Electron identification .................... 62

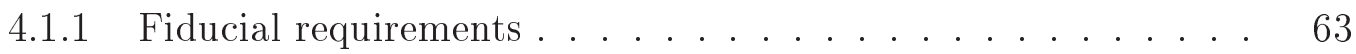

4.1.2 Tracking and calorimeter ID cuts ............ 64

4.1.3 Conversion removal . . . . . . . . . . . . . 69

4.2 Muon identification .......................... 71

4.2.1 Fiducial requirements . . . . . . . . . . . . . . . . 72

4.2.2 Track, calorimeter, and muon stub ID cuts . . . . . . . 76

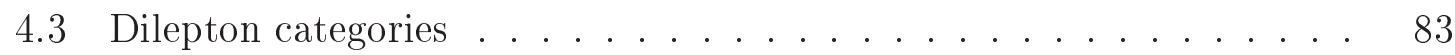

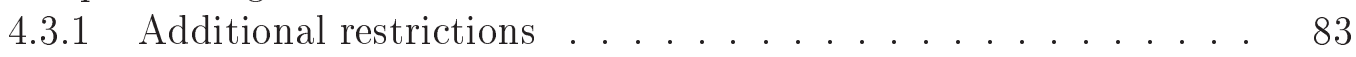

4.4 Cosmic ray removal . . . . . . . . . . . . . . . . . 85

4.5 Like-sign/opposite-sign event separation . . . . . . . . . . . . 87

4.6 Isolation . . . . . . . . . . . . . . . . . . . . . . . . . . . . . . . . . .

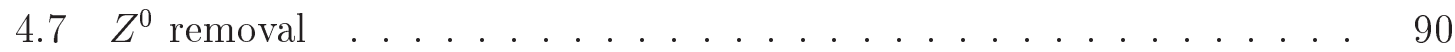

4.8 Missing energy . . . . . . . . . . . . . . . . . . . . . 96

4.9 Jet identification . . . . . . . . . . . . . . . . . . . . . . 97

4.10 Summary . . . . . . . . . . . . . . . . . . . 98

5 Data Results $\quad 101$

5.1 Datasets ............................. 101

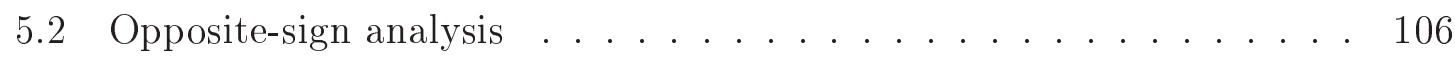

5.3 Like-sign analysis . . . . . . . . . . . . . . . . 110

5.4 Summary .............................. 116

6 Monte Carlo Acceptance $\quad 117$

6.1 Minimal Supersymmetric Standard Model . . . . . . . . . . . . 118

6.2 Cross section for $\tilde{g} \tilde{g}$ production . . . . . . . . . . . . . . 123

6.3 Monte Carlo samples . . . . . . . . . . . . . . . . . 125

6.4 Lepton ID efficiencies . . . . . . . . . . . . . . . . . . . . . . . . . . . . . . . 134

6.5 Same-sign dilepton acceptance summary . . . . . . . . . . . . 139

7 Systematic Uncertainty 143

7.1 Parton distribution function . . . . . . . . . . . . . . . . . 144

7.2 Initial and final state gluon radiation . . . . . . . . . . . . . 144 
$7.3 \quad Q^{2}$ scale dependence $\ldots \ldots \ldots \ldots \ldots \ldots \ldots$

7.4 Jet energy scale dependence . . . . . . . . . . . . . . . . . 147

7.5 Luminosity . . . . . . . . . . . . . . . . . . . . . . . 149

7.6 Remaining sources of error . . . . . . . . . . . . . 150

7.6.1 Photon conversion removal . . . . . . . . . . . . . . . 150

7.6 .2 Lepton ID efficiency . . . . . . . . . . . . . . . . . 151

7.6 .3 Lepton isolation . . . . . . . . . . . . . . . . . 151

7.6 .4 Tracking efficiency . . . . . . . . . . . . . . 152

7.6.5 Trigger efficiency . . . . . . . . . . . . . . . 152

7.7 Total systematic uncertainty . . . . . . . . . . . . . . . 153

8 Background Estimate $\quad 155$

8.1 Background samples . . . . . . . . . . . . . . . . . 157

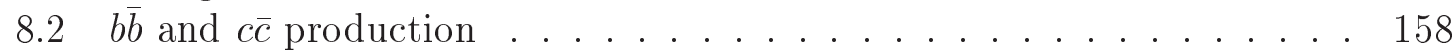

8.3 Drell-Yan . . . . . . . . . . . . . . . . . . . . 168

8.4 Dibosons $(W W, W Z, Z Z) \ldots \ldots \ldots \ldots \ldots \ldots \ldots$

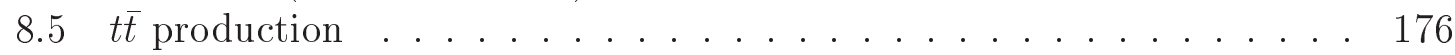

8.6 Fake leptons . . . . . . . . . . . . . . . . . . . . 177

8.7 Background contribution summary . . . . . . . . . . . . . 182

9 Results and Conclusions 184

9.1 Statistical analysis . . . . . . . . . . . . . . . . . . . 184

9.2 Prospects for Run II . . . . . . . . . . . . . . . . . . . . . . 191

9.3 Conclusions . . . . . . . . . . . . . . . . . . . . . . . 199

$\begin{array}{ll}\text { Bibliography } & 201\end{array}$ 


\section{List of Figures}

2.1 Extrapolation of the inverse gauge couplings, $\alpha_{i}^{-1}$ from the electroweak energy scale up to the Planck scale. The dashed lines represent the Standard Model inverse couplings, which come close to unification at the very high energy but do not share a common intersection point. The solid lines show the evolution when additional physics enters the picture just above the current energy reach of present accelerators. At the unique intersection around $10^{16} \mathrm{GeV}$, the three forces are revealed to be simply low-energy versions of a single unified force. . . . . . . .

2.2 Corrections to the Higgs mass for any fermions (f) or scalars $(\mathrm{S})$ in the theory. Taken from Ref. [3]. . . . . . . . . . . . . .

2.3 Second-order correction diagrams for a heavy fermion (F) which couples indirectly to the Higgs. Taken from Ref. [3]. . . . . . . . . . .

2.4 At lowest order, gluino pair production follows from an excited gluon state produced by gluon fusion or quark-antiquark annihilation. Higher order Feynman diagrams also contribute significantly to the cross section. 28

2.5 Feynman diagram showing the gluino decay to top and scalar top and the subsequent decay of the stop to a charm quark and neutralino. . . 29

2.6 Feynman diagram for the leptonic decay of the top quark. . . . . . 30

3.1 Layout showing the Fermilab accelerator stages leading up to injection of the beams into the Tevatron. . . . . . . . . . . . . 38

3.2 Single quadrant schematic of the CDF detector. . . . . . . . .

3.3 Schematic for one of the two identical SVX barrels. Three silicon microstrip detectors are joined to create a ladder, and twelve ladders arranged in $\phi$ at equal radius form a layer. There are four layers, with radii from 2.86 to $7.87 \mathrm{~cm}$. The bulkhead provides structural support as well as channels for cooling the system. Readout of the system is performed at the outer ends. . . . . . . . . . . . . 
3.4 Layout of an individual SVX ladder. Three silicon detectors are mounted onto a lightweight Rohacell support and wirebonded to form a single electrical unit, with effective strip lengths of $25 \mathrm{~cm}$. Radiation-hard readout electronics are attached at the far end of the ladder. . . . . .

3.5 An individual cell of the Central Tracker Chamber. As a track passes through and ionizes the gas, the drift electrons are collected by the sense wires, and the charge distribution measures the track position. Potential wires aid in shaping the electric field. . . . . . . . . .

3.6 End view of the Central Tracking Chamber, showing the 9 superlayers (0-8) and the $45^{\circ}$ tilt of the drift cells with respect to the radial direction. The larger axial cells occupy the even layers, while the smaller stereo cells comprise the odd layers. . . . . . . . . . . .

3.7 Two images showing the calorimeter structure. Along the direction of the beam, as shown on the left, we see a $15^{\circ}$ wedge in $\phi$, with the CHA mounted directly outside the CEM. In the transverse view to the right, the $\eta$ coverage is shown. The detector is segmented in units of $\Delta \eta=0.11$, and the resulting towers (0-9) point back to the interaction region. The Central Muon chambers are also shown. . . . . . . . .

3.8 Diagram of a central muon tower. In the CMU, this subtends an angle of $4.2^{\circ}$ and consists of 16 drift cells which are $2.26 \mathrm{~m}$ long. A muon track passes through, ionizing the gas, and timing information on the hits allows reconstruction of the track. Any ambiguity in the track position is resolved by offsetting each pair of layers by $2 \mathrm{~mm}$. . . . .

$3.9 \eta-\phi$ coverage of the CDF central muon detector systems. The $\phi$ gaps in the CMP correspond to gaps in the return yoke, while the large $\phi$ gaps in the CMX occur where the CMX intersects with the floor (large gap) and the Tevatron (small gap). . . . . . . . . . . .

4.1 Distributions of central electron (TCE and LCE) identification variables, where the dashed line shows where the cut is placed. . . . . .

4.2 Conversion electrons are identified by searching for two electron tracks which are closely spaced in $r-\phi(|\delta(r-\phi)<0.3 \mathrm{~cm}|)$ and in $\theta(\mid \delta \cot \theta<$ $0.06 \mid)$. Also shown is the two-track invariant mass. After application of the angular cuts, the conversion radius distribution nicely illustrates the location of the beampipe, the SVX and VTX cabling and supports, and the CTC inner radius. Taken from Ref. [26]. . . . . . . . . . .

Distributions of CTC track pseudorapidity $(\eta)$ and azimuthal angle $(\phi)$ for all inclusive muons (TCM, CMX, and CMI) identified by the Lepton ID requirements. The CMI distribution is enlarged by a factor of 10 to enhance its visibility. . . . . . . . . . . . 
4.4 Distributions of CTC track $\eta$ and $\phi$ for all TCM muons, separated according to the type of muon stub associated with the track: matching CMU and CMP stubs, CMU only, and CMP only. Gaps in the CMP coverage appear as clusters of CMU only muons, and vice versa. . . .

4.5 Distributions of central muon (CMU, CMP, and CMUP) identification variables, where the dashed line shows where the cut is placed. . . . .

4.6 Distributions of central muon extension (CMX) identification variables, where the dashed line shows where the cut is placed. . . . . . . .

4.7 Distributions of central minimum ionizing particle (CMI) identification variables, where the dashed line shows where the cut is placed. . . . .

4.8 Distributions of the calorimeter isolation for electrons originating from three different sources: $W \rightarrow e \nu_{e}, b \rightarrow c e \nu_{e}$, and $\tau \rightarrow e \nu_{e} \nu_{\tau}$. These were produced from $t \bar{t}$ Monte Carlo and only require that the electrons be within the fiducial region of the detector and have $E_{T}>20 \mathrm{GeV}$. .

4.9 Distributions of the calorimeter isolation for muons originating from three different sources: $W \rightarrow \mu \nu_{\mu}, b \rightarrow c \mu \nu_{\mu}$, and $\tau \rightarrow \mu \nu_{\mu} \nu_{\tau}$. These were produced from $t \bar{t}$ Monte Carlo and only require that the muons be within the fiducial region of the detector and have $p_{T}>20 \mathrm{GeV}$. .

4.10 Distributions of the invariant mass for dielectron events in Run I data. These are shown for opposite-sign events which pass the lepton ID, cosmic ray, and isolation cuts. The dashed lines show the range of masses removed by the $Z^{0}$ mass cut. . . . . . . . . . . . .

4.11 Distributions of the invariant mass for dimuon events in Run I data. These are shown for opposite-sign events which pass the lepton ID, cosmic ray, and isolation cuts. The dashed lines show the range of masses removed by the $Z^{0}$ mass cut. . . . . . . . . . . . .

4.12 Distributions of the photon + dilepton invariant mass for $e e$ and $\mu \mu$ events in Run I data. These are shown for opposite-sign events which pass the lepton ID, cosmic ray, isolation, and invariant mass cuts. The dashed lines show the range of masses removed by the radiative $Z^{0}$ mass cut. . . . . . . . . . . . . . . .

4.13 Comparison of the raw and corrected transverse energy for the highest $E_{T}^{\text {raw }}$ and second highest $E_{T}^{\text {raw }}$ jets, for opposite-sign dilepton events which pass the lepton ID and isolation cuts. . . . . . . . . .

5.1 Event display for the same-sign dilepton event 61074/103772. The top diagram presents a transverse view of the tracks in the CTC. The red arrow indicates the direction of the $\mathbb{E}_{T}$. The lego display in the lower diagram illustrates the distribution of EM (purple) and hadronic (cyan) calorimeter energy. . . . . . . . . . . . . . . . 114 
5.2 Event display for the same-sign dilepton event 57621/45230. The top diagram presents a transverse view of the tracks in the CTC. The red arrow indicates the direction of the $\mathbb{E}_{T}$. The lego display in the lower diagram illustrates the distribution of EM (purple) and hadronic (cyan) calorimeter energy. . . . . . . . . . . . . . .

6.1 In the absence of $R$-parity conservation, proton decay is mediated via an intermediate squark. . . . . . . . . . . . . .

6.2 Gluino pair production mechanisms: a) Born-level $g g$ diagrams; b) Born-level $q \bar{q}$ diagrams; c) Vertex corrections from QCD and SUSY; d) Bremsstrahlung diagrams. Taken from Ref. [42]. . . . . . . . . . .

6.3 (a) Dependence of the total cross section for $\tilde{g} \tilde{g}$ production at the Tevatron $(\sqrt{s}=1.8 \mathrm{TeV})$ upon the choice of $Q^{2}$ scale. The sensitivity to $Q / m_{\tilde{g}}$ is shown for different choices of PDF and mass parameters $m_{\tilde{g}}=250 \mathrm{GeV}$ and $m_{\tilde{q}}=200 \mathrm{GeV}$. (b) $K=\sigma_{N L O} / \sigma_{L O}$ is shown for $Q / m_{\tilde{g}}=2,1,1 / 2$ (corresponding to the upper, middle, and lower curves) using the GRV parton density function. Taken from Ref. [42].

6.4 Calculation of $\tilde{g} \tilde{g}$ cross section (in pb) from PROSPINO. Values were calculated using the CTEQ 4M PDF and default $Q^{2}$ scale for a range of squark masses from 200 to $1000 \mathrm{GeV}$. The scale at right represents the total number of expected $\tilde{g} \tilde{g}$ events in Run I, regardless of decay mode or detection efficiency. . . . . . . . . . . . . . . .

6.5 Calculation of $\tilde{g} \tilde{g}$ cross section (in pb) from PROSPINO. Values were calculated using the CTEQ 4M PDF and squark mass of $1 \mathrm{TeV}$. The central curve is for the default QCD renormalization scale, while the upper and lower come from increasing and decreasing, respectively, $Q^{2}$ by a factor of 4 . On the scale at right is the total number of $\tilde{g} \tilde{g}$ events expected for the Run I integrated luminosity. . . . . . . . . . . .

6.6 Combinations of gluino and stop mass explored in $\tilde{g} \rightarrow t \overline{\tilde{t}}$ Monte Carlo. The solid diagonal line indicates the kinematic limit on available stop masses due to the $175 \mathrm{GeV}$ top quark mass. The neutralino mass was generally related to the stop mass via $m_{\tilde{\chi}_{1}^{0}}=m_{\tilde{t}}-20 \mathrm{GeV}$, except where $m_{\tilde{t}} \leq 40 \mathrm{GeV}$. . . . . . . . . . . . . . . . . . .

6.7 Lepton ID efficiencies for the lepton classes TCE, LCE, TCM, and CMX for three ranges of calorimeter isolation. The open points are $Z^{0} \rightarrow l l$ Monte Carlo and the closed points are data. The dashed lines show the 3 isolation bins, and the error bars are statistical only. . . .

6.8 Cumulative efficiency at each stage of the same-sign dilepton analysis for $\tilde{g} \rightarrow t \overline{\tilde{t}}$ events in Monte Carlo. These results cover only the samples where $200 \leq m_{\tilde{g}} \leq 320, m_{\tilde{t}}=m_{\tilde{g}}-180 \mathrm{GeV}$ and $m_{\tilde{\chi}_{1}^{0}}=m_{\tilde{t}}-20 \mathrm{GeV}$

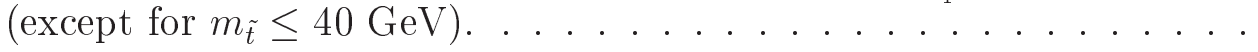


8.1 Detection efficiency for TCE category electrons produced by the decay of $W$ 's and $b$ quarks in $t \bar{t}$ Monte Carlo events, as a function of lepton isolation. . . . . . . . . . . . . . . .

8.2 Leading order Feynman diagram for Drell-Yan production of lepton pairs in a hadronic collider event. Next-to-leading order forms include initial-state gluons which provide sufficient jet activity to pass the 2-jet cut. Here we consider only direct production of electrons and muons (although $\tau^{+} \tau^{-}$final production also contributes. . . . . . . . 169

8.3 Feynman diagram for $W W$ diboson production. Analogous diagrams exist for $W Z$, while $Z Z$ production proceeds via a diagram analogous to the left one involving boson-fermion couplings. . . . . . . . . .

8.4 Feynman diagram for $W$ boson production associated with 3 or more

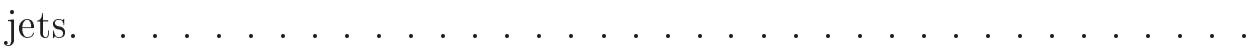

$9.195 \%$ Confidence Level limits on $\tilde{g} \tilde{g} \rightarrow(t \tilde{t})(t \tilde{t})$ compared to the nextto-leading order (NLO) theoretical calculation of $\sigma_{\tilde{g} \tilde{g}}$ from PROSPINO. 189

9.2 The upper limit curve is the Run I limit, based on the 3 observed events in the data. The lower limit curve is based on the presumption of only 1 observed event in $106.1 \mathrm{pb}^{-1}$. In the second case, the extrapolated limit on $m_{\tilde{g}}$ would have been $\approx 196 \mathrm{GeV} \ldots \ldots$. . . . . . . .

9.3 Comparison of the Run I and Run II NLO cross sections for gluino pair production $\left(\sigma_{\tilde{g} \tilde{g}}\right)$ at the Tevatron. The increase in center of mass energy from $1.8 \mathrm{TeV}$ to $1.96 \mathrm{TeV}$ boosts $\sigma_{N L O}$ by $35-70 \%$ over the range of gluino masses shown. The scale at right shows the corresponding number of $p \bar{p} \rightarrow \tilde{g} \tilde{g}+X$ events expected to be produced in $2 f b^{-1}$ of luminosity during the first stage of Run II. . . . . . . . . . . . .

9.4 Comparison of the cross section for gluino pair production at Run I energy $\left(E_{c m}=1.8 \mathrm{TeV}\right)$ and Run II energy $\left(E_{c m}=1.96 \mathrm{TeV}\right) . \ldots$

9.5 The missing $E_{T}$ significance is plotted versus $E_{T}$ for dielectrons from $t \bar{t}$ in PYTHIA Monte Carlo (top) and from $Z^{0}+2$ jet background in VECBOS (bottom). In both cases, the lepton ID selection and standard missing $E_{T}$ cuts have been applied. The diagonal line and line at $\phi=3$ are for reference only. (Taken from Ref. [76].) . . . . . .

9.6 Monte Carlo distributions of $H_{T}$ for dilepton events from $t \bar{t}$ and background processes which pass the Run I analysis criteria. The Run I data are also shown. (Taken from Ref. [75].) . . . . . . . . . . . .

9.7 Rough estimate of the potential for the same-sign dilepton analysis for setting a limit on $m_{\tilde{g}}$ in Run II $\left(2 f b^{-1}\right)$. This assumes the same conditions (observed signal and estimated background) as those used to calculate the Run I limit. 


\section{List of Tables}

2.1 Experimental limits on the quark and lepton masses. The lepton masses benefit from precision experiments, while quark measurements suffer from indirect methods and model dependent factors. . . . . . .

2.2 Comparison of the intrinsic spins of the Standard Model particles and their supersymmetric partners. All other properties (charge, color, mass) are shared by the partners in a multiplet. . . . . . . . . . .

2.3 All available decay modes from top pair production and their branching ratios. The modes involving tau are considered separately in top analyses, so the branching fractions for channels involving only electrons and muons are also shown. . . . . . . . . . . . .

3.1 Summary of pseudorapidity coverage and angular segmentation for all Central, Plug, and Forward electromagnetic and hadronic calorimeters. 50

3.2 Summary of the event rates and deadtimes for the CDF trigger stages. 58

3.3 Single tower energy thresholds for the Level 1 calorimeter triggers in Run Ia and Ib for all electromagnetic and hadronic calorimeter systems. 59

4.1 Electron identification requirements, with distinctions between tight (TCE) and loose (LCE) category electrons specified. . . . . . . . . . 67

4.2 TCM and CMX muon identification requirements . . . . . . . . 77

4.3 Minimum ionizing (CMI) particle identification requirements . . . . 79

4.4 There are 12 distinct categories which arise from the pairings of the five classes of leptons (TCE, LCE, TCM, CMX, and CMI), and the requirement that at least one lepton be tight. . . . . . . . . 84

4.5 Photon identification requirements for the radiative $Z^{0}$ cut. . . . . . 94

4.6 Summary of all selection requirements which will be applied to Run I data and Monte Carlo events in the same-sign dilepton analysis. . . 100

5.1 Number of events in the Run Ia and Run Ib inclusive electron and muon datasets. Also shown are the number of events which pass each of the requirements comprising the Lepton ID stage of the analysis. . 104 
5.2 Multiple trigger paths at Level 3 for high- $p_{T}$ electrons. An electron is included in the Stream A inclusive electron sample if selected by any of the above triggers. . . . . . . . . . . . . 105

5.3 Results from the opposite-sign dilepton analysis of Run I data. . . . 107

5.4 Comparison between this analysis and the original top dilepton analysis for events which have passed all cuts except the 2-jet cut. In the top dilepton note [28], the trilepton event has been assigned to the TCMTCM category. Here, we have moved it to the separate trilepton category.108

5.5 Discrepancies in the beam constrained momentum measured by the top dilepton analysis and our opposite-sign analysis arise due to changes in the beam spot definition. This causes the newly calculated $\mathbb{E}_{T}$ to pass the missing energy cut. . . . . . . . . . . . . 109

5.6 Results from the like-sign dilepton analysis of Run I data. . . . . . . 111

5.7 Kinematic properties of the two same-sign dilepton events and one trilepton event. For event 57621/45230, the second set of lepton variables is for the $\mu^{-}$. The second muon $\left(\mu^{+}\right)$has $p_{T}=21 \mathrm{GeV}$ and is located within the primary jet $\left(j_{1}\right) \ldots \ldots \ldots \ldots$

6.1 Chiral supermultiplets in the Minimal Supersymmetric Standard Model.119

6.2 Gauge supermultiplets in the Minimal Supersymmetric Standard Model.119

6.3 Results from like-sign analysis of Monte Carlo samples for $\tilde{g} \tilde{g} \rightarrow t t+$ $X \rightarrow l l+X$ for a range of gluino, stop, and neutralino masses. . . . . 132

6.4 Results from opposite-sign analysis of Monte Carlo samples for $\tilde{g} \tilde{g} \rightarrow$ $t t+X \rightarrow l l+X$ for a range of gluino, stop, and neutralino masses. .

6.5 Comparison of the selection efficiencies in Monte Carlo for our $\tilde{g} \tilde{g}$ samesign dilepton analysis versus an opposite-sign analysis of PYTHIA $t \bar{t}$ events. Each efficiency is based on the number of events which have passed all of the preceding cuts. The difference in overall acceptance is due mainly to the branching ratio of $\tilde{g} \tilde{g}$ to like-sign top. . . . . . .

6.6 Comparison of lepton ID efficiencies for $Z^{0} \rightarrow l l$ Run Ib data and ISAJET+QFL Monte Carlo for the five lepton classes. The errors are statistical only. . . . . . . . . . . . . . .

6.7 Comparison of lepton ID efficiencies in Monte Carlo versus data as a function of calorimeter isolation for the TCE, LCE, TCM, and CMX lepton classes. For minimum ionizing muons (CMI), all allowed isolation values are grouped together. . . . . . . . . . . .

6.8 Scale factors for correcting the same-sign dilepton event acceptance to account for differences in lepton ID efficiencies between Run I data and Monte Carlo. For minimum ionizing muons (CMI), all allowed isolation values are grouped together, while all other lepton classes are separated into 3 isolation bins. . . . . . . . . . . . . . 
6.9 Results of the same-sign analysis of all $\tilde{g} \tilde{g}$ Monte Carlo samples after application of the event-by-event scale factors to correct for the differences in lepton ID efficiency between MC and data. The overall acceptance rate, including the scale factors, is calculated in the column

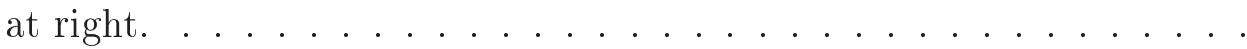

6.10 Summary of the same-sign dilepton acceptance rates calculated from Monte Carlo simulation of $\tilde{g} \rightarrow t \overline{\tilde{t}}$. . . . . . . . . . . . 142

7.1 Dependence of acceptance on choice of PDF . . . . . . . . . . 144

7.2 Dependence of acceptance on IS and FS gluon radiation . . . . . . . . 145

7.3 Dependence of acceptance on the choice of the QCD momentum transfer scale. . . . . . . . . . . . . . . 147

7.4 Dependence of acceptance on jet energy scale . . . . . . . . . . 148

7.5 Summary of all sources of systematic error. The overall systematic uncertainty on the dilepton acceptance is obtained by adding all errors in quadrature. . . . . . . . . . . . . . . . . .

8.1 Like-sign analysis of quark pair production $(b \bar{b}, c \bar{c})$ samples which include only direct production. . . . . . . . . . . . . . .

8.2 Like-sign analysis of quark pair production $(b \bar{b}, c \bar{c})$ samples which involve $q \bar{q}$ creation from initial state gluon radiation. . . . . . . . . .

8.3 Like-sign analysis of quark pair production $(b \bar{b}, c \bar{c})$ samples which involve $q \bar{q}$ creation from final state gluon radiation. . . . . . . . . . .

8.4 Opposite-sign analysis of quark pair production $(b \bar{b}, c \bar{c})$ samples which include only direct production. . . . . . . . . . . . . . . .

8.5 Opposite-sign analysis of quark pair production $(b \bar{b}, c \bar{c})$ samples which involve $q \bar{q}$ creation from initial state gluon radiation. . . . . . . . .

8.6 Opposite-sign analysis of quark pair production $(b \bar{b}, c \bar{c})$ samples which involve $q \bar{q}$ creation from final state gluon radiation. . . . . . . . . . . 167

8.7 Like-sign analysis of all Drell Yan $\gamma$ and $Z^{0}$ samples. . . . . . . . . . 171

8.8 Opposite-sign analysis of all Drell Yan $\gamma$ and $Z^{0}$ samples. . . . . . . . 172

8.9 Summary of the expected Drell-Yan contribution to the same-sign dilepton signal. The errors are statistical only, based on the assumption one event would have passed the analysis despite the fact that no events passed the $\mathbb{E}_{T}$ cuts. . . . . . . . . . . . 173

8.10 Like-sign analysis of diboson $W W, W Z$ and $Z Z$ samples. . . . . . . 175

8.11 Opposite-sign analysis of diboson $W W, W Z$ and $Z Z$ samples. . . . . 175

8.12 Summary of the expected diboson contribution to the same-sign dilepton signal. The errors are statistical only. For the $W W$ and $Z Z$ backgrounds, the errors are based on the assumption that for similar sized samples, one event would have passed the analysis. . . . . . . 176 
8.13 Like-sign analysis of $t \bar{t}$ samples, with and without gluon radiation incorporated into the ISAJET simulations. . . . . . . . . . . . . 178

8.14 Opposite-sign analysis of $t \bar{t}$ samples, with and without gluon radiation incorporated into the ISAJET simulations. . . . . . . . . . . 178

8.15 Estimated contribution from fake lepton events for any opposite-sign or like-sign top dilepton analysis in Run I, using the "fakeable jet" probability and $W+$ multijet sample. The number of events from fakes is shown after all dilepton selection cuts except for the 2 - jet cut and after all cuts. . . . . . . . . . . . . . .

8.16 Estimated contribution to the same-sign top dilepton channel from all Standard Model background sources. The statistical errors are added in quadrature. . . . . . . . . . . . . . . . . . .

9.1 Upper limit on $\sigma_{\tilde{g} \tilde{g}}$ calculated from the acceptances for the various Monte Carlo samples. . . . . . . . . . . . . . . . 188

9.2 Comparison of the mechanical specifications for the SVX' (Run I) and SVX II (Run II) detectors. . . . . . . . . . . . . . . . . . . 195 
Science is magic that works.

Kurt Vonnegut, Cat's Cradle 


\section{Chapter 1}

\section{Introduction}

The purpose of particle physics is to discover the basic building blocks of the universe and understand the ways in which those pieces interact with each other. This process is constantly evolving, as the physical and mathematical tools involved become more sophisticated.

Much like how the Newtonian law of gravitation was shown by Einstein to be an approximation of the more fundamental theory of relativity, the current theory of particle physics, known as the Standard Model, is believed to be a low-energy version of a more general and wider-reaching theory. Despite the wonderful agreement between the Standard Model and the body of results from particle accelerators around the world, physicists are constantly seeking out discrepancies, ways in which the theory is broken, or hints of something that lies beyond our present understanding. These make up the "New Physics" which we are continually searching for, but any refinement to the Standard Model must solve its troubling aspects without adding needless complexity.

The most promising candidate for such an extension is the theory of Supersymmetry, or SUSY. One of the most nagging questions we have in particle physics is: Why do particles have mass and what determines the masses that they have? Supersymmetry answers this by introducing a fundamental symmetry between the particles which constitute matter and the particles which carry the forces between them.

In the Standard Model, all particles obtain their masses by interacting with a 
proposed particle, known as the Higgs, which remains as the final piece of the Standard Model to be discovered. The field associated with Higgs permeates all of spacetime, and in popular descriptions it has been likened to a molasses through which everything must travel. How strongly a particle interacts with the Higgs determines whether it is massive or it is light. Theoretical calculations involving properties of the Higgs, however, result in undesirable infinities which cannot be successfully "swept under the rug" in the traditional ways that led to viable descriptions of electromagnetism and strong interactions by QED and QCD, respectively.

Supersymmetry solves these problems by introducing a partner for every known Standard Model particle, where the only distinction between the particle and its superpartner is a difference in intrinsic spin. This effectively doubles the number of fundamental particles, yet none of these new superpartners have been observed thus far. This fact indicates that Supersymmetry is somehow broken at the energy scales we are familiar with, while remaining perfectly valid at higher energies. Without understanding the mechanism behind this, we are left with an elegant theory that explains several issues in the Standard Model in a relatively simple manner, yet predicts a plethora of new particles and provides little direction as to where to look for them. We can only speculate about their masses and decay modes, although the amount of available parameter space to explore provides plenty of options for experimental searches and topics for young graduate students to build a dissertation around.

In this analysis, we search for direct pair production of the gluino $(\tilde{g})$, supersymmetric partner to the gluon, in proton-antiproton collisions. We assume one possible scenario for SUSY where the superpartner of the top quark, known as scalar top or stop $(\tilde{t})$, is the lightest scalar quark. In this case, the only decay mode available to the gluino involves the top quark, which was first observed at Fermilab in 1994. The CDF experiment, of which Johns Hopkins is a member institution, has gained much expertise in detecting the various top quark event signatures, and a search for SUSY which incorporates top is well-suited to take advantage of this.

Chapter 2 describes the theoretical motivation behind the search: what we know from the Standard Model, the reasons why Supersymmetry is so popular, and the 
event topology we expect to observe. In Chapter 3, we describe the Tevatron accelerator and the CDF experiment at Fermilab, with special attention given to those detector components crucial to the analysis.

The event selection process is presented in finer detail in Chapter 4, and the results from analyzing the data from Run I (1992-1996) at CDF follow in Chapter 5. Next, we focus on the Monte Carlo simulation of the $\tilde{g} \rightarrow t \overline{\tilde{t}}$ process and calculation of the event acceptance rate (Chapter 6), followed by an estimate of the systematic uncertainty in this measurement (Chapter 7). Finally, we estimate the contribution from background processes to the observed signal in Chapter 8. A discussion of the results and some concluding remarks are presented in Chapter 9. 


\section{Chapter 2}

\section{Theoretical motivation}

\subsection{What is everything made of?}

At the beginning of the new millennium, the field of particle physics is in relatively good shape. Compared to even the start of the 20th century, we have come quite a long way in understanding the basic composition of matter and the nature of the universe. Our efforts are all in pursuit of answering the fundamental question: what is everything made of and how does the universe work?

Ancient Greek philosophers were among the first to describe the world in terms of atoms - the smallest, indivisible pieces of which all matter, and indeed all reality, is composed. This picture changed relatively little in the following centuries. Even by the late 1800 's, although many of the chemical elements had been discovered, the concept of atoms was just that - a theoretical construct mainly useful as a bookkeeping device for describing chemical reactions. No one knew if atoms truly existed or, if so, what they actually consisted of.

\subsubsection{Atoms and their constituents}

The discovery of the electron by J.J. Thompson in 1897 (barely 100 years ago now) was the first hint that objects smaller than the hydrogen atom existed. Although Thompson could only measure its charge to mass ratio, he was able to infer that its 
mass was small. (The ratio of the electron mass to the hydrogen mass is $1 / 1800$.) The oil drop experiment by R. Millikan in the early 1910's conclusively demonstrated that electric charge is quantized and appears in multiples of an elementary charge $e$, the charge of the electron.

Ordinary matter appeared to be electrically neutral, although with enough energy atoms could be given a net charge through ionization. If atoms did indeed contain electrons, an equal number of oppositely charged particles would also have to inhabit the atom. Thompson proposed that the electrons floated around in a cloud of positive charge, "like raisins in a pudding."

Experiments by Rutherford, Geiger, and Marsden in 1909 found that when they bombarded a gold foil with alpha particles (understood by Rutherford to be electrically charged helium atoms and later discovered to be the helium nucleus, ${ }_{2}^{4} \mathrm{He}$ ) emitted from radioactive material, an unexpected number were scattered at large angles. Such behavior ruled out a uniform charge density and indicated that atoms instead consist of mostly empty space with an extremely dense center. The atom appeared to be not unlike a miniature solar system, with a nucleus of positively-charged protons at the core and electrons circling in well-defined orbits.

We now know that atoms have sizes on the order of $10^{-10} \mathrm{~m}$ (1 Angstrom, or $\dot{\mathrm{A}}$ ), while the nucleus is $1 / 100000$ th smaller $\left(10^{-15} \mathrm{~m}=1\right.$ femtometer, or fm). If an atom were expanded to the size of a football field, the nucleus would be on the order of a millimeter across. Because the size of the atom is a thousand times smaller than the wavelength of visible light (4000-7000 $\dot{\mathrm{A}})$, our experience is far removed from the "graininess" of matter. In order to probe these tiny distance scales, machines of higher and higher energy must be constructed so that the effective wavelength of our "microscope" is small enough to "see" the structure we are interested in.

Electromagnetism, however, raised some troublesome questions about the proposed atomic structure. First, an unknown mechanism held the nucleus together against the repulsive Coulomb forces between the like-charged protons packed tightly within the nucleus. Also, Maxwell's equations predicted that accelerating a charged particle causes it to radiate light. As an electron circles the nucleus, radiation should be continuously emitted, leading the electron to lose energy and spiral down into the 
nucleus. The apparent stability of the atom contradicted this scenario, but it was not clear what prevented its predicted collapse.

By the mid to late 1920's, several properties of the proton and electron had been measured. The young field of quantum mechanics had introduced the concept of the wave-particle duality of nature, and the electron energy levels and their stability could be explained in terms of orbits which allowed for standing electron waves. Quantized energy levels accounted for the observed atomic transmission and absorption spectral lines.

Meanwhile, experiments by Curie and Joliot using radioactive materials to bombard a beryllium target had revealed a new, deeply penetrating form of radiation. Chadwick showed that this new particle, the neutron, appeared similar in mass to the proton but would necessarily be electrically neutral to be capable of passing through several inches of lead. A second constituent of the nucleus had been discovered. Although the "strong" forces involved were not quite understood, it was believed that neutrons were the glue that held the nucleus together against the repulsive force between the protons.

Returning to radioactivity, nuclear beta decay - the decay of a neutron to a proton and electron, and source of the idea that the nucleus contained electrons exhibited behavior fundamentally different from other types of radiation. The process of alpha decay clearly conserved energy and momentum by radiating a photon if the alpha particle received less than the maximum possible energy. Beta decay, on the other hand, exhibited a spectrum of electron energy without the accompaniment of a gamma ray. Pauli suggested that the missing energy was carried away by an invisible, light, neutral particle, which Fermi dubbed the neutrino $(\nu)$. Fermi suggested the presence of a "weak" force which mediated the decay of the neutron. It would be nearly two decades before conclusive evidence of the neutrino was discovered by Reines and Cowan.

The then-current structure of particle physics could be summarized by:

$$
\left(\begin{array}{c}
p^{+} \\
n
\end{array}\right)\left(\begin{array}{c}
\nu_{e} \\
e^{-}
\end{array}\right)
$$


plus the photon, $\gamma$, which acts as the carrier (quantum) of the electromagnetic force. The origins of the strong and weak forces remained a mystery. During the next few decades, this simple picture changed significantly as hints of new physics began to be seen.

\subsubsection{Antimatter and cosmic rays}

In the late 1920's, Dirac extended the classical quantum mechanical description of the electron to include relativity. In addition to components corresponding to the spin orientation of the electron (discussed in the next section), his relativistic wave equation included a troublesome "negative energy" solution that was difficult to interpret. Rather than discard this result, Dirac explored the implications of such a solution and proposed that a "sea" of negative energy states existed. A uniform density of such states throughout space would be undetectable, although it appeared to be possible to create an observable positive energy state by adding an energy of at least $2 m_{e} c^{2}$. This would leave a "hole" in the sea which would behave like a positively charged electron.

In 1911, physicists using the earliest particle detectors discovered cosmic rays, high-energy particles which continuously bombard the Earth's atmosphere. Three years after Dirac's discovery, work by Anderson revealed an object identical in mass to the electron except having a positive charge. The positron, as it came to be known, was the first of many antimatter particles to be discovered.

The positron perfectly filled the role of the Dirac negative energy state, and the previous electron-hole scenario was reinterpreted as the creation of an electronpositron pair from a photon with energy equal to the mass energy of the pair $\left(2 m_{e} c^{2}\right)$. The converse process is also possible. An electron and positron can scatter off each other through the electromagnetic force, but if they become too close, they will annihilate and create a burst of energy. This principle is true of all matter particles and their antimatter counterparts and is the basis of many accelerator designs, which typically involve the collision of $e^{+}$and $e^{-}$, or $p^{+}$and $p^{-}$, beams.

The photon had been identified as the quantum of the electromagnetic force, and 
the early success of Quantum Electrodynamics (QED) had motivated theorists to develop a quantum theory of the nuclear force. The massless photon corresponded to an infinite range force, so the short distance scales of the nuclear force implied the exchange of a massive particle, according to the Yukawa theory. Rough calculations predicted a mass on the order of $150 \mathrm{MeV}$. With an intermediate mass between those of the electron and proton, such a particle was dubbed a meson.

Subsequent studies of cosmic rays in 1936 by Anderson revealed a new object with mass of around $100 \mathrm{MeV}$. This was quickly dubbed the "mu meson," or muon, in anticipation of it being the Yukawa particle. Years later, in 1947, a second meson was identified and named the pion. The pion was closer to the prescribed Yukawa mass at $140 \mathrm{MeV}$ and decayed to produce a muon. The muon was recognized as having all the characteristics of the electron, only it is 200 times heavier. The existence of the muon, neither a fundamental constituent of matter nor a carrier of force, seemed completely unnecessary. This prompted I.I. Rabi to ask the question contemplated by many physicists: "Who ordered that?"

\subsubsection{The particle zoo}

During the 1950's, a plethora of new particles was discovered. The pion was only the first of dozens (and eventually hundreds) of strongly interacting baryons and mesons to appear. All of these were unstable, challenging the very notion of what it meant to be a "fundamental" particle.

In 1947, the kaon (or $V$ particle) was first observed in cosmic rays using a cloud chamber. Within a few years, a sufficient number of events had been observed to characterize the particle. The production mechanism appeared to involve the strong force, but the long lifetime $\left(10^{-10}\right)$ was more indicative of the weak decay of the muon and pion. The high preponderance of events containing two decay patterns could only be explained by pair production, which strongly hints of an underlying conservation principle. Gell-Mann proposed a new quantum number, termed strangeness $(S)$. Conservation of strangeness was imperfect, unlike any other conservation law, in that it appeared to conserved for strong interactions but violated in the weak decay. In 
addition to $S$, another useful concept is the hypercharge, defined as $Y=S$ for mesons and $Y=S+1$ for baryons. Hypercharge has no meaning for leptons.

The key to understanding the complex array of new particles was to organize them according to their hypercharge and isospin. The proton and neutron, for instance, form an isospin doublet. They are nearly identical in all respects except for charge, and as such can be thought of as two states of the same particle, similar to the two different spin states of an electron: spin up $\left(I_{z}=+1 / 2\right)$ for the proton, spin down $\left(I_{z}=-1 / 2\right)$ for the neutron. What Gell-Mann, Nakano, and Nishijima discovered was a relationship between the charge, isospin, and hypercharge:

$$
Q=I_{z}+Y / 2
$$

Also, the mesons and baryons naturally arranged themselves into sets of eight, or octets:

$$
\begin{aligned}
& n^{0} \quad p^{+} \quad Y=1 ; \quad I_{z}=-1 / 2,+1 / 2 \\
& \Sigma^{-} \quad \Sigma^{0}, \Lambda^{0} \quad \Sigma^{+} \quad Y=0 ; \quad I_{z}=-1,0,+1 \\
& \Xi^{-} \quad \Xi^{0} \quad Y=-1 ; \quad I_{z}=-1 / 2,+1 / 2 \\
& K^{0} \quad K^{+} \quad Y=1 ; \quad I_{z}=-1 / 2,+1 / 2 \\
& \pi^{-} \quad \pi^{0}, \eta^{0} \quad \pi^{+} \quad Y=0 ; \quad I_{z}=-1,0,+1 \\
& \bar{K}^{-} \quad \bar{K}^{0} \quad Y=-1 ; \quad I_{z}=-1 / 2,+1 / 2
\end{aligned}
$$

The significance of the octet structure was not immediately obvious, and some of the above particles had not yet been discovered when these ideas were first proposed. The GNN theory slowly gained acceptance as, one by one, the missing pieces of the structure were confirmed. 


\subsubsection{The quark model}

Early particle accelerators include the Cockroft-Walton and van de Graaff, as well as the cyclotrons designed by Lawrence. With the advent of these new machines, experimenters reached the point where a proton beam could be collided with a heavy nucleus target to yield pions, which would then be directed onto a secondary target. Studies of this nature, led by Fermi at the University of Chicago cyclotron, revealed the existence of resonances, short-lived excited states of the nucleon. This is characteristic of a system with internal structure and degrees of freedom, similar to the electronic energy levels in the atom. Indeed, the regular octet patterns in the GNN model are reminiscent of the organization of the periodic table.

In 1964, Gell-Mann introduced the quark model, which fundamentally altered our understanding of particle physics. Previous approaches, such as the Fermi-Yang and Sakata theories, had described the recently discovered baryons and mesons as combinations of the stable baryons $p, n$, and $\Lambda$. Gell-Mann carried the idea a step further, suggesting that all strongly acting particles, including protons and neutrons, are composed of smaller, more fundamental objects: the up $(u)$, down $(d)$, and strange (s) quarks.

The most controversial aspect of the theory was that quarks carry charges of $+2 / 3,-1 / 3$, and $-1 / 3$. Grouping these into pairs and triplets produced particles with integer charge, and the spin orientation of the quarks (spin-1/2) allowed for the creation of half-integer spin baryons and integer spin mesons. The complete absence of any fractionally charged particles in Nature, despite intensive efforts to locate them, convinced many in the field that quarks were nothing but a convenient mathematical construct with little physical basis.

At the Stanford Linear Accelerator Center (SLAC) in 1967, a group led by Friedman, Kendall, and Taylor employed the high-energy electron beam to explore deep inside the proton structure. As an analogue to the Rutherford probe of the atom, these scattering experiments uncovered pointlike objects within the proton that matched the characteristics of the proposed up and down quarks. Yet, the validity of fractional charges remained to be proven. 
By late 1972, results emerged from the Gargamelle experiment at CERN, the particle physics laboratory on the French-Swiss border near Geneva. An enormous 5-m long, 12,000-L bubble chamber with an 800-ton magnet was used to study nucleonneutrino interactions. On the order of a billion neutrinos passed through the detector per second, yet the event yield was only 1 per minute. Out of half a million events photographed, 2000 involved an inelastic collision with a proton or neutron. This proved to be enough. The pointlike structure observed by SLAC was confirmed, as were the fractional charges. The number of "free" quarks inside the nucleon was calculated to be three, as expected, and the quark theory finally gained broad acceptance.

\subsubsection{The final pieces of the puzzle}

Shortly thereafter, the simultaneous discovery of the $J / \psi$ resonance in 1974 at Brookhaven and SLAC was an unexpected turn of events. Subsequently, several additional mass resonances $\left(\psi^{\prime}\right.$, et al.) were found at regularly spaced intervals, as was a new set of mesons $(\chi)$. The $J / \psi$, with a mass of $3.1 \mathrm{GeV}$, could not be explained by the three quark model. Earlier discovered resonances were understood in terms of linear combinations of quark-antiquark pairs - u $\bar{u}, d \bar{d}, s \bar{s}-$ and the existing possibilities had been exhausted.

Years earlier, theorists studying the weak force had theorized the existence of a fourth quark, named "charm". The known particles, plus the proposed $c$ quark, could be paired into doublets:

$$
\left(\begin{array}{l}
u \\
d
\end{array}\right) \quad\left(\begin{array}{l}
c \\
s
\end{array}\right) \quad\left(\begin{array}{l}
e^{-} \\
\nu_{e}
\end{array}\right) \quad\left(\begin{array}{l}
\mu^{-} \\
\nu_{\mu}
\end{array}\right)
$$

The weak force mediates changes between the doublets through interactions such as $\mu^{-} \rightarrow e^{-}+\bar{\nu}_{e}+\nu_{\mu}$ and $u \rightarrow d+e^{-}+\bar{\nu}_{e}$. At the time, the only particle without a partner was the $s$ quark, but the introduction of charm completed the picture. Although qualitative in nature, the symmetry argument was quite compelling, and the newly found $J / \psi$ was a strong candidate for $c \bar{c}$.

The theoretical suspicions were corroborated in 1976 by the discovery of the $D$ and $F$ (or as currently identified, $D_{s}$ ) mesons. With masses in the 1.8 to $2.0 \mathrm{GeV}$ range, 
approximately half that of the $J / \psi$, these particles were identified as the pairings of the $c$ quark with the three lighter quarks. A rough calculation yielded a charm mass of $\sim 1.5 \mathrm{GeV}$, while the masses of the $u$ and $d$ are very small and the mass of the $s$ quark is a few hundred $\mathrm{MeV}$.

During the same year, the SPEAR electron-positron machine at SLAC produced a third lepton, the tau. At $1.8 \mathrm{GeV}$, the $\tau$ mass is 17 times heavier than the muon and 3500 times larger than the electron. Although there was no question about the existence of a tau neutrino, experimental confirmation of $\nu_{\tau}$ (by the DONUT collaboration at Fermilab in 2001) lagged 25 years behind the $\tau$ observation.

The discovery of $\tau$ by Perl and associates at SLAC opened the door for further quark searches, and Lederman's group at the $400 \mathrm{GeV}$ proton-proton machine at Fermilab almost immediately found the upsilon resonance, $\Upsilon$. This $9.5 \mathrm{GeV}$ meson was instantly recognized as the $q \bar{q}$ state of a fifth quark, the $b$ ("bottom" or "beauty") quark, with a mass around 4-5 GeV.

A sixth quark, $t$ ("top" or "truth"), was essential to completion of the third generation quark doublet. The trend in quark and meson masses convinced some that a $15 \mathrm{GeV}$ top or $30 \mathrm{GeV} t \bar{t}$ resonance was within reach. In fact, 15 years of experimental searches ensued, culminating with the announcement in 1994 by Fermilab experiments CDF and D0 of evidence for a $175 \mathrm{GeV}$ top in the $1.8 \mathrm{TeV}$ proton-antiproton collisions at the Tevatron.

The final pieces of the puzzle had fallen into place, and the Standard Model of particle physics appeared complete. One may wonder if this process continues unabated. Perhaps additional undetected quarks and leptons remain, as there are no strong theoretic constraints on the number of families. Searches for a fourth generation quark at present accelerators have thus far turned up nothing [1], but the required energy is conceivably just beyond our reach. Another alternative is that quarks and leptons are composite objects and the array of "elementary" particles arises from the various combinations of more fundamental components. Then again, three generations of quarks and leptons may be the only option for a fully viable universe, with the requirement of an additional sector of particles to explain the properties of the observed particle spectrum. The possibilities surrounding the last 
option are the focus of this analysis.

\subsection{The Standard Model}

\subsubsection{Particles and spin}

The Standard Model encompasses our current understanding of the fundamental building blocks of matter and the rules which govern how those pieces interact. The current description of the universe is populated by twelve matter particles and their antiparticles, known collectively as fermions, plus four carriers of force, or bosons. (Note that this count ignores the gravitational force and color factors for quarks and gluons.) The major distinguishing characteristic between fermions and bosons is the fundamental quantity known as spin. Spin is an intrinsic property of a particle, on par with charge or mass, and is interpreted as an intrinsic angular momentum, similar to the motion of a tiny rotating top.

Just as charge is quantized in units of the electron charge, angular momentum and spin are measured in half units of the Planck constant $\hbar$. The component of a particle spin along the direction of its momentum may take any of $2 j+1$ values, between $-j$ and $j$, in steps of 1 . For example, an electron has spin $\frac{1}{2}$ and has orientations of spin up $\left(+\frac{1}{2}\right)$ and spin down $\left(-\frac{1}{2}\right)$. The direction of particle spin compared to its momentum determines the helicity, or "handedness". A right-handed particle has spin parallel to the momentum, while for a left-handed one, the two are antiparallel. Most quarks and leptons appear in both helicity states. Assuming that they are massless, neutrinos possess the unique characteristic of existing in only one state: all neutrinos are left-handed and all antineutrinos are right-handed.

Particles with half-integer spin $\left(\frac{1}{2}, \frac{3}{2}, \ldots\right)$ obey Fermi-Dirac statistics. No two identical fermions may exist in the same quantum state of position, momentum, spin, etc., and the state of a system must be antisymmetric under the exchange of any pair of fermions. This idea was originally useful in explaining the electron shell structure of the atom and its absorption and emission spectra. All of the classically identified constituents of matter (electrons, protons, neutrons, and their constituent quarks) 
are fermions.

Particles with integer spin $(0,1, \ldots)$ obey Bose-Einstein statistics, where a system is symmetric under the exchange of any two bosons. This implies that any number of Bose particles may exist in the same quantum state at a given time. The best example of this phenomenon is the laser, where a vast number of photons in the same state are concentrated in a single pulse. Another is the recently observed Bose-Einstein condensate state of matter in helium at near-absolute zero temperatures. In addition to the photon of electromagnetism, the quanta of all the other classical fields are bosons.

\subsubsection{Fermions and matter}

Fermions are subdivided into 6 leptons and 6 quarks. The leptons include the electron and its neutrino $\left(\nu_{e}\right)$, plus the muon and tau and their associated neutrinos $\left(\nu_{\mu}, \nu_{\tau}\right)$. The $\mu$ and $\tau$ are similar to the electron in most respects, only heavier and unstable. The leptons are categorized into three families:

$$
\left(\begin{array}{c}
e^{-} \\
\nu_{e}
\end{array}\right) \quad\left(\begin{array}{c}
\mu^{-} \\
\nu_{\mu}
\end{array}\right) \quad\left(\begin{array}{c}
\tau^{-} \\
\nu_{\tau}
\end{array}\right), \quad Q=\left(\begin{array}{c}
-1 \\
0
\end{array}\right)
$$

To be completely pedantic, we note that the doublets above should carry an $L$ subscript to indicate that the constituents are the left-handed helicity states. In the absence of a right-handed neutrino, the corresponding right-handed doublet is replaced by singlet states for the electron, muon, and tau: $e_{R}, \mu_{R}, \tau_{R}$.

Similarly, the quarks are separated into three families. The up and down quarks, which combine to form the proton and neutron, make up the first family while their more massive and short-lived cousins fill the other two:

$$
\left(\begin{array}{l}
u \\
d
\end{array}\right) \quad\left(\begin{array}{l}
c \\
s
\end{array}\right)\left(\begin{array}{l}
t \\
b
\end{array}\right), \quad Q=\left(\begin{array}{c}
+\frac{2}{3} \\
-\frac{1}{3}
\end{array}\right)
$$

Here, both helicity states are allowed, so the doublets may be left-handed or righthanded. 


\subsubsection{Bosons and forces}

There exist four fundamental interactions between these particles. Although gravity was the first to be discovered and studied, its origin is ironically the least understood. It is the weakest force, by many orders of magnitude, and has a negligible effect between individual particles. Yet gravity is the force closest to our own experience. This is due to the fact that it has infinite range and its effect is purely additive. There is only one type of gravitational "charge," and the force is always attractive.

The electromagnetic force first described by Maxwell is now known to be transmitted by the photon $(\gamma)$ and affects all particles that carry an electric charge. This gives rise to the several phenomena of charged particles that are useful in particle physics: ionization of matter as a charge passes through, arching trajectories in a magnetic field, and the radiation of light when accelerated. The EM force is much stronger than gravity, and the range is also infinite. However, the two types of charge, positive and negative, exist in roughly equal proportions so that bulk matter is electrically neutral. Therefore its overall influence is muted to a large extent, although there are many everyday situations where one encounters the effects of electricity and magnetism.

Unlike gravity and electromagnetism, the strong force has a severely limited range. Over distance scales on the order of that of the nucleon, it becomes the strongest force, hence the name. It is the mechanism which binds quarks together to form protons, neutrons, and all other hadrons. Residual effects hold the nucleus together against the repulsive forces between protons. Carried by the massless gluon, the strong force influences only quarks, while leptons are unaffected.

Quarks each contain one of three color charges (labeled "red", "green", and "blue", for instance), while antiquarks have anticolor. The gluon carries two charges (one color and one anticolor) and allows a quark to transition from one color state to another. The three colors combine to form a "colorless" state, thus the analogy with the primary colors. The other possible colorless state is the combination of a colored quark with an antiquark of the corresponding anticolor.

The nature of the strong force requires that observable objects have no net color. 
This explains the grouping, or confinement, of quarks into baryons $\left(q_{1} q_{2} q_{3}\right.$; protons, neutrons, etc.) and mesons $\left(q_{1} \bar{q}_{2} ; \pi^{ \pm}, \pi^{0}\right.$, etc.). Single-quark states may exist only over very short time scales and immediately draw out quark-antiquark pairs from the energy of the vacuum, a process known as hadronization or fragmentation. In fact, any attempt to extract a quark from a bound state results in the conversion of energy into new $q \bar{q}$ pairs. In high energy particle collisions, this process is readily apparent; the production of a quark often leads to a shower of hadronic particles, known as a jet. In collider events containing a jet, it is a difficult process to conclusively identify the original quark by measuring the properties of the jet contents, although useful information can be extracted.

Finally, the weak interaction was originally identified with several different processes, including beta decay in radioactive elements and the decay of the neutron, pion, muon, and other particles. The weak force is also the mechanism by which the neutrino interacts with ordinary matter and is associated with small distance scales and interaction cross sections (in other words, a very small likelihood of interaction). These interactions may involve leptons, quarks, or both, and are characterized by long lifetimes $\left(\sim 10^{-10} \mathrm{sec}\right)$.

Original attempts to describe the weak force as a point-like interaction proved bothersome. The cross section for scattering of an neutrino and electron, for instance, was calculated to be proportional to the square of the energy of the interaction: $\propto E^{2}$. The difficulty here is that such theories are not renormalizable, meaning that troubling infinities arise due to a dependence upon the large momentum cutoff in the theory.

A solution was found in the introduction of heavy intermediary bosons which transmitted the force. Even though the original objects are not energetic enough to directly produce these massive bosons, such particles can be virtually created for an extremely short period of time and not violate conservation of energy. This is because the Heisenberg Uncertainty Principle limits the precision to which one can simultaneously measure the energy and time of a reaction:

$$
\Delta E \Delta t \geq \hbar
$$

As long as the amount by which energy is not conserved, $\Delta E$, occurs over a time 
scale $\Delta t$ which satisfies $\Delta E \Delta t \leq \hbar$, the virtual particle may be created.

The carriers of the weak force, the $W^{ \pm}$and $Z^{0}$ turned out to more massive than expected (80.4 and $91.2 \mathrm{GeV}$, respectively) and were not experimentally verified until 1983 at CERN. The production of a heavy intermediate particle demands that $\Delta t$ be relatively small, and this resolves the seemingly point-like quality of the interaction with the desire for renormalizability. Furthermore, the probability of creating a virtual boson is inversely related to the mass, and this explains why the lifetimes of particles which decay via the weak force are orders of magnitude longer than those which decay via electromagnetism or the strong force.

\subsection{What's wrong with the SM}

Despite all the successes of the Standard Model, many fundamental questions still present themselves. What mechanism gives rise to the three family structure of quarks and leptons, and are there more, yet undiscovered families? Do neutrinos have mass, and can they change flavors - between an electron type and a muon type, for instance - thereby violating the separate conservation of electron, muon, and tau lepton number? Why are matter and antimatter not equally balanced in the universe? What are the mechanisms behind the breaking of this and other symmetries?

One of the most pressing concerns in particle physics is the origin of mass. In the Standard Model, the quark and lepton masses are free parameters and must be measured by experiment. More desirable would be a theory which predicts the masses and describes the observed hierarchy. With the discovery of the top quark, even more questions were raised. Specifically, why is the top quark so much more massive than the other quarks, or any other particle for that matter? The range of quark masses spans 5 orders of magnitude, and the electron is another factor of 10 smaller than the lightest quarks.

Table 2.1 shows the most recent estimates of the quark and lepton masses. Note that quark confinement prevents direct observation of the mass, so the accuracy of these measurements is restricted by the indirect methods used. The masses of the lightest quarks actually have the largest percentage uncertainty. The lepton masses, 


\begin{tabular}{|cc|cc|}
\hline \hline Quark & Mass (MeV) & Lepton & Mass (MeV) \\
\hline$d$ & $3-9$ & $e$ & 0.511 \\
$u$ & $1-5$ & $\mu$ & 105.7 \\
$s$ & $75-170$ & $\mu$ & \\
$c$ & $1150-1350$ & & 1777 \\
$b$ & $4000-4400$ & $\tau$ & \\
$t$ & $174300 \pm 5100$ & & \\
\hline \hline
\end{tabular}

Table 2.1: Experimental limits on the quark and lepton masses. The lepton masses benefit from precision experiments, while quark measurements suffer from indirect methods and model dependent factors.

on the other hand, are precisely known, with the measurement error less than the least significant digit shown.

Another weakness of the Standard Model is gravity. The successful quantum mechanical description of the electromagnetic and weak forces (QED) and the strong force (QCD) stands in stark contrast to the fact that a satisfactory quantum treatment of gravity has yet to be found. A proposed spin-2 boson, known as the graviton, is associated with the gravitational field.

String theory has been the focus of theoretical efforts for the past twenty years and holds much promise of combining general relativity and quantum mechanics. The general idea is that matter is composed not of point particles but extremely tiny vibrating strings which form the fabric of spacetime. One advantage that string theory has is that Supersymmetry, the idea which forms the basis of this search (described below), appears to be a natural consequence of the theory. However, extrapolation of the theory down to the energy scales accessible at present accelerators has proven to be quite difficult. This has so far precluded any predictive power, which is essential to the ultimate adoption of any new theory no matter how mathematically beautiful. 
Attempts to unify the other three forces have been somewhat more successful. Weinberg and Salam showed in the late 1960's that the electromagnetic and weak forces are actually low-energy versions of a combined electroweak interaction at energies achieved by current accelerators. One would hope to combine these with the strong force in a Grand Unified Theory (GUT), where all three forces observed at the electroweak scale are in fact manifestations of a single unified force at higher energy.

In fact, the strengths of the EM, strong, and weak forces are dependent upon the energy of the interaction. The coupling constant $\alpha_{i}$ is a gauge of the relative strength of a particular interaction, and measurements of $\alpha_{i}$ for the three forces have been performed at energies up to the electroweak scale. It is possible, though, to extrapolate these relative strengths up to higher energy scales, as shown in Figure 2.1. (Actually, the inverses of the gauge couplings are shown.) In the Standard Model case, the coupling constants come tantalizingly close in the $10^{13}-10^{16} \mathrm{GeV}$ range. With the insertion of additional physics processes at an intermediate energy, a common intersection can be achieved around $10^{16} \mathrm{GeV}$, which would imply a unified force around that energy. One must be careful to realize that this prediction is made for energies 14 orders of magnitude higher than currently observable. On the other hand, the beauty of this possibility is rather seductive.

\subsection{The Higgs particle}

The question then becomes: what manner of new physics enters the picture, and at what energy scale? In the Weinberg-Salam electroweak model, the gauge bosons $\left(W^{ \pm}\right.$and $\left.Z^{0}\right)$ gain mass through "spontaneous symmetry breaking," which comes about by introducing a scalar field known as the Higgs. The Standard Model Higgs potential takes the form

$$
V(H)=m_{H}^{2}|H|^{2}+\frac{\lambda}{2}|H|^{4}
$$

Precision measurements at LEP and the Tevatron indirectly place limits on the Higgs mass. Current experimental data suggests that the Higgs mass is less than $235 \mathrm{GeV}$ at the $95 \%$ confidence level [2]. 


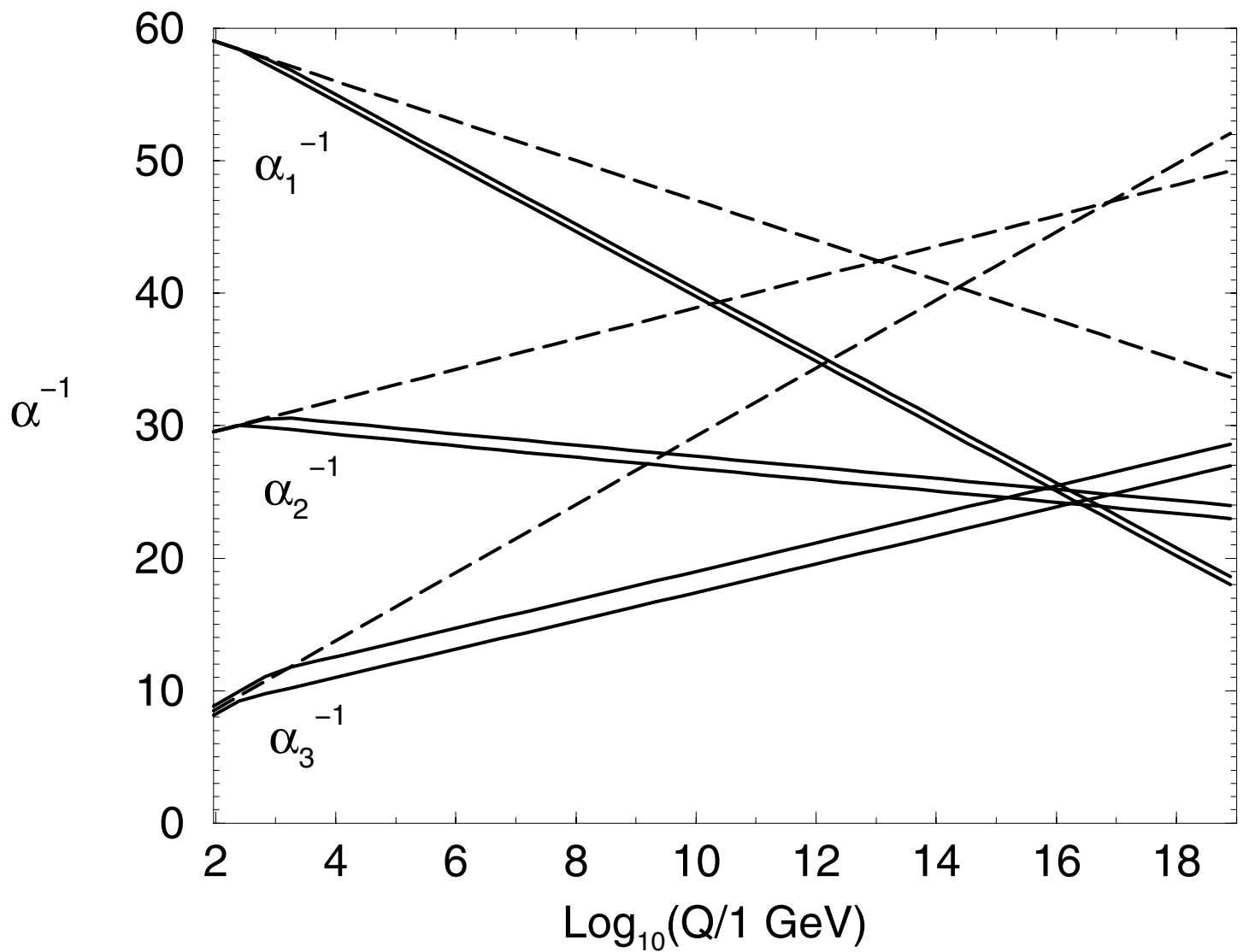

Figure 2.1: Extrapolation of the inverse gauge couplings, $\alpha_{i}^{-1}$ from the electroweak energy scale up to the Planck scale. The dashed lines represent the Standard Model inverse couplings, which come close to unification at the very high energy but do not share a common intersection point. The solid lines show the evolution when additional physics enters the picture just above the current energy reach of present accelerators. At the unique intersection around $10^{16} \mathrm{GeV}$, the three forces are revealed to be simply low-energy versions of a single unified force. 


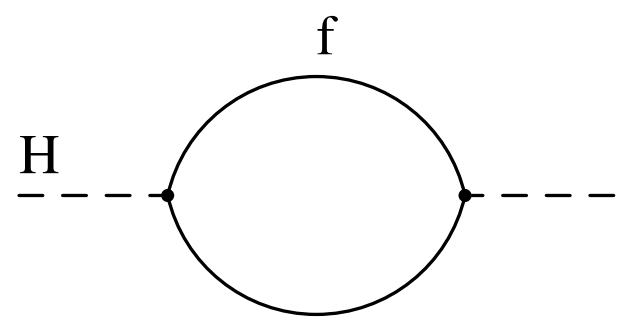

(a)

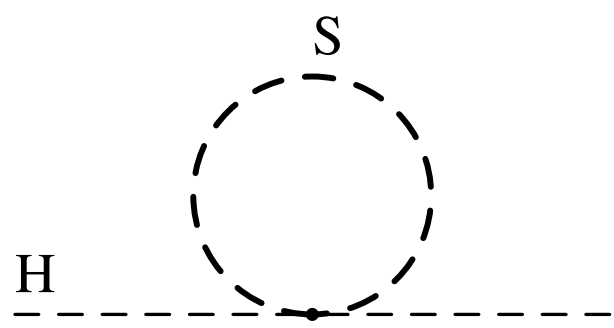

(b)

Figure 2.2: Corrections to the Higgs mass for any fermions (f) or scalars $(\mathrm{S})$ in the theory. Taken from Ref. [3].

However, a problem arises when quantum loop corrections are made to the Higgs mass calculation. The self-energy of the Higgs contains effects from the fact that quantum fluctuations allow the Higgs to occasionally produce other particles on a very brief time scale before a return to its original state. Such processes do not violate the Uncertainty Principle, mentioned in Section 2.2.3, but do alter the observed properties of the Higgs. For any massive fermions or scalar particles (bosons) that couple directly to the Higgs, Feynman diagrams such as those shown in Figure 2.2 contribute corrections to the Higgs mass of the form:

$$
\begin{aligned}
\Delta m_{H}^{2} & =\frac{\left|\lambda_{f}^{2}\right|}{16 \pi^{2}}\left[-2 \Lambda_{\mathrm{UV}}^{2}+6 m_{f}^{2} \ln \left(\Lambda_{\mathrm{UV}} / m_{f}\right)+\cdots\right] \\
\Delta m_{H}^{2} & =\frac{\lambda_{S}}{16 \pi^{2}}\left[\Lambda_{\mathrm{UV}}^{2}-2 m_{S}^{2} \ln \left(\Lambda_{\mathrm{UV}} / m_{S}\right)+\cdots\right]
\end{aligned}
$$

where $\Lambda_{\mathrm{UV}}$ is the large momentum cutoff for the integral used in the computation. This upper bound may be interpreted as the energy at which new physics enters the picture and changes the nature of the interaction, rendering meaningless any contribution from above that energy scale.

One likely scenario is that $\Lambda_{\mathrm{UV}}$ corresponds to the Planck scale, the energy regime at which the quantum effects of gravity become comparable to those of the other forces. The Planck mass (energy) may be derived from Newton's gravitational constant:

$$
m_{\text {Planck }}=1 / \sqrt{8 \pi G_{\text {Newton }}}=2.4 \times 10^{18} \mathrm{GeV}
$$




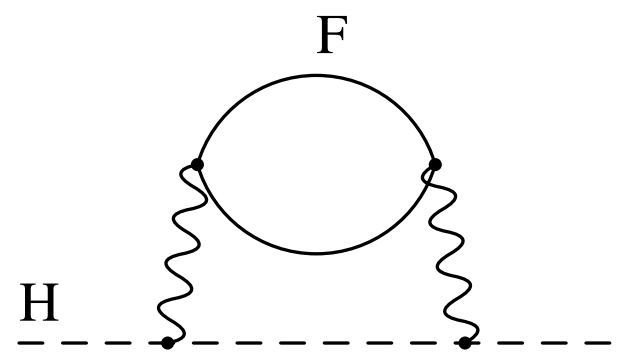

(a)

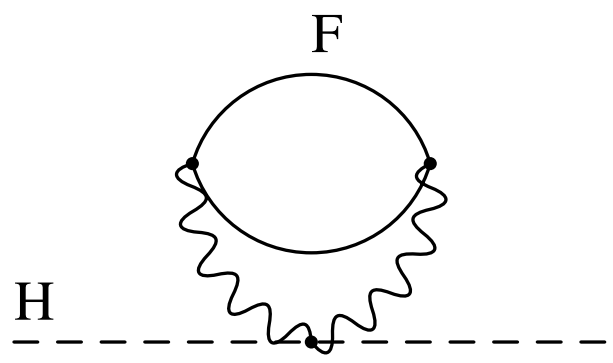

(b)

Figure 2.3: Second-order correction diagrams for a heavy fermion (F) which couples indirectly to the Higgs. Taken from Ref. [3].

If $\Lambda_{\mathrm{UV}}$ is truly on par with the Planck scale, the corrections to the Higgs mass will be enormous, possibly 16 orders of magnitude greater than the experimental value. This discrepancy (and the huge difference between the electroweak and Planck scales in general) has been termed the gauge hierarchy problem. A modified approach to performing the integration makes it possible to drop the $\Lambda_{\mathrm{UV}}^{2}$ terms, but the problem does not completely disappear. One must believe in the complete absence of new particles to be discovered in the energy region between our current reach of $\mathcal{O}(100 \mathrm{GeV})$ and the Planck scale. There is no theoretical motivation for such a scenario, and the idea runs contrary to the past 50 years of experience.

Even if one postulates that heavy particles exist which do not couple directly to the Higgs, problems with the theory still emerge. Second-order diagrams, shown in Figure 2.3, may be constructed for a heavy fermion $\mathrm{F}$ that couples indirectly to the Higgs:

$$
\Delta m_{H}^{2}=x\left(\frac{g^{2}}{16 \pi^{2}}\right)^{2}\left[c_{0} \Lambda_{\mathrm{UV}}^{2}+48 m_{F}^{2} \ln \left(\Lambda_{\mathrm{UV}} / m_{F}\right)+\cdots\right]
$$

Again, a similar problem occurs with the dependence on $\Lambda_{\mathrm{UV}}$ and any potentially heavy mass $m_{F}$. The contradiction between these apparently large corrections and an expected (and desirable) TeV scale Higgs is cause for alarm.

Several possibilities have been proposed for avoiding the hierarchy problem. Some theories, such as technicolor, suggest that the Higgs particle is actually composite. 
The self-energy scale issues are avoided, and the idea has experimental implications that may be pursued by the next generation of accelerators. Others, like topcolor, argue that the Higgs does not exist at all. One intriguing solution is that spacetime consists of more than the standard $(3+1)$ dimensions, and the comparative weakness of gravity is due to the propagation of its lines of force into these extra hidden dimensions. The high-energy cutoff would lie just above the current TeV scale, rather than at the Planck scale, and there would be no more particles to discover. These theories are beyond the reach of this paper, but are interesting proposals nonetheless.

\subsection{Supersymmetry}

Given the fact that the family structure remains to be understood and that there is no theoretical argument against more particles lying just beyond our reach, it is unlikely that no further physics awaits in the next 16 orders of magnitude in energy scale. One of the most promising theories for extending our understanding of particle physics beyond the Standard Model is known as Supersymmetry [4].

If the Higgs truly exists, some mechanism is essential to rescue the theory from runaway corrections to the Higgs mass. In examining Eq. 2.3 and Eq. 2.4, note the relative sign difference between the quadratic terms $\left(\propto \Lambda_{\mathrm{UV}}^{2}\right)$ and between the logarithmic terms $\left(\propto \ln \left(\Lambda_{\mathrm{UV}} / m\right)\right)$. As mentioned before, the quadratic parts can be eliminated, using a technique known as dimensional renormalization. The problem reduces to one involving only the logarithmic terms: one proportional to $\left|\lambda_{f}^{2}\right| m_{f}^{2} \ln \left(\Lambda_{\mathrm{UV}} / m_{f}\right)$ and the other proportional to $\lambda_{S} m_{S}^{2} \ln \left(\Lambda_{\mathrm{UV}} / m_{S}\right)$.

Due to the relative negative sign, alternating contributions from fermion and scalar loops counterbalance each other, and under the right circumstances could be designed to completely offset. In order for this to occur, two assertions have to be made. First, for every massive scalar there must exist a fermion with degenerate mass (and vice versa). Furthermore, for each partnership the constants describing the coupling of the Higgs to the scalar and to the fermion must satisfy $\lambda_{S}=\left|\lambda_{f}^{2}\right|$. Under these circumstances, calculation of $\Delta m$ for any scalar loop would be exactly counteracted by that for a corresponding fermionic loop. Witten and others showed that if two 
scalars with $\lambda_{S}=\left|\lambda_{f}^{2}\right|$ are assigned to each lepton and quark in the Standard Model, the first-order loops of Figure 2.2 balance out [5].

Similar contributions dependent upon $\Lambda_{\mathrm{UV}}$ stem from higher-order Feynman diagrams, and the real difficulty lies in finding a way for the bosonic and fermionic adjustments to the Higgs mass to systematically cancel at all orders. Arbitrarily imposing conditions and fine tuning a theory should be avoided at all cost. A neat and tidy cancellation of this magnitude, though, can be a consequence of an underlying symmetry. Before elaborating further, we should revisit the symmetries of the Standard Model.

In electromagnetism, the matter-antimatter symmetry relates particles, such as the electron and positron, which are identical aside from their electrical charge. (Actually, the crucial quantity is the hypercharge $Y$, but charge is a more accessible concept. For leptons, $Y$ is meaningless anyway.) Charge conjugation is a discrete operation which converts from one charge state to the other, and the underlying mathematical symmetry group of EM is known as $U(1)_{Y}$.

The symmetry of the electroweak extension is $S U(2)_{L} \times U(1)_{Y}$. Here the weak interaction couples the fundamental particles into the left-handed doublets shown earlier and allows a rotation from one component to the other. $t \rightarrow b W$, for instance, is allowed since the top mass is large enough to create a real $W$. Virtual $W$ 's are involved in other processes, but these are accompanied by suppression factors. Due to mixing effects between the down-type quarks $(d, s$, and $b)$, the weak force facilitates transitions between families.

Finally, the strong force is governed by the mathematics of $S U(3)_{c}$. This is the color symmetry of the quarks. Each quark type is replaced by a triplet composed of the three flavor states. The gluon, actually an octet of color-anticolor operator combinations $\left(C_{1} \bar{C}_{2}\right)$, performs the transformations among the distinct color states for a given quark.

The theory of Supersymmetry, or "SUSY" for short, resolves the issues surrounding the Higgs mass by adopting a fundamental symmetry between fermions and bosons. Supersymmetry postulates the existence of a superpartner for every particle in the Standard Model, and each particle and its superpartner form a supermulti- 


\begin{tabular}{lr|rr}
\multicolumn{2}{c|}{ Standard Model } & \multicolumn{2}{c}{ SUSY } \\
\hline e $, \mu, \tau, \nu$ & $\tilde{e}, \tilde{\mu}, \tilde{\tau}, \tilde{\nu}$ & $S=0$ \\
$q$ & $S=1 / 2$ & $\tilde{q}$ & $S=0$ \\
$g$ & $S=1$ & $\tilde{g}$ & $S=1 / 2$ \\
$\gamma, W, Z$ & $S=1$ & $\tilde{\gamma}, \tilde{W}, \tilde{Z}$ & $S=1 / 2$ \\
$H^{0}, h^{0}, A^{0}, H^{ \pm}$ & $S=0$ & $\tilde{H} \tilde{h}^{0}, \tilde{A}^{0}, \tilde{H}^{ \pm}$ & $S=1 / 2$
\end{tabular}

Table 2.2: Comparison of the intrinsic spins of the Standard Model particles and their supersymmetric partners. All other properties (charge, color, mass) are shared by the partners in a multiplet.

plet, the analogue of the weak doublet. In the language of quantum mechanics, the transformation, or rotation, from one particle type to the other is performed by an operator $Q$ such that:

$$
\begin{aligned}
& Q \mid \text { boson }\rangle=\mid \text { fermion }\rangle \\
& Q \mid \text { fermion }\rangle=\mid \text { boson }\rangle
\end{aligned}
$$

Each member of a supermultiplet shares all the properties of its counterpart except for a difference in spin of $1 / 2$ unit. The spin-1/2 quarks and leptons receive bosonic partners with integral spin 0; the gauge particles - photons, gluons, $W$ and $Z$ bosons, and the Higgs - acquire spin-1/2 fermion superpartners. The full particle spectrum is summarized in Table 2.2. Some parameterizations of supersymmetry require additional Higgs particles $\left(h^{0}, A^{0}, H^{ \pm}\right)$and their corresponding superpartners.

A supersymmetric particle is designated with a tilde, so the partners of the gluon and top quark are denoted by $\tilde{g}$ and $\tilde{t}$. Because the left- and right-handed states of quarks and leptons transform differently in the Standard Model, each is paired separately with a superpartner. For instance, the top quark helicity states $t_{L}$ and $t_{R}$ have partners indicated by $\tilde{t}_{L}$ and $\tilde{t}_{R}$. Since the $\tilde{t}$ has spin 0 , only one helicity state exists, so the $L$ and $R$ subscripts refer to the helicity of the top quark partner and not to that of the scalar top. 
The nomenclature of SUSY continues the tradition of assigning somewhat whimsical names to new particles that began with the quarks: "strange," "charm," etc. The newly predicted superpartners of the fermions are technically known as "scalar fermions," or sfermions. In general, the prefix $s$ - is added to the Standard Model name, leading to terms such as squark and slepton, selectron and stop. The partners of the gauge bosons are dubbed gauginos, and the individual names are formed by attaching the suffix -ino to the SM name. This gives rise to the gluino, photino, wino, zino, and Higgsino.

In the $S U(2)_{L} \times U(1)_{Y}$ electroweak theory, the spin-1 gauge particles are $W^{+}, W^{-}$, $W^{0}$, and $B^{0}$. Below the energy scale at which electroweak symmetry is broken, the neutral states $W^{0}$ and $B^{0}$ mix to form the observed photon and $Z^{0}$ mass eigenstates. In unbroken supersymmetry, a corresponding mixing occurs between the wino $\left(\tilde{W}^{0}\right)$ and bino $\left(\tilde{B}^{0}\right)$ to create a massless photino $(\tilde{\gamma})$ and a zino $\left(\tilde{Z}^{0}\right)$ with mass equal to $m_{Z^{0}}$.

The situation becomes slightly more complicated because SUSY is a broken symmetry for energies below the TeV scale, for reasons explained below. The $\tilde{W}^{0}, \tilde{B}^{0}$, and neutral Higgsinos mix to form a series of neutral mass states referred to as neutralinos $\left(\chi_{1}^{0}, \chi_{2}^{0}, \chi_{3}^{0}, \chi_{4}^{0}\right)$. The $\tilde{W}^{ \pm}$and charged Higgsino states also mix to create the charged mass eigenstates known as charginos $\left(\chi_{1}^{ \pm}, \chi_{2}^{ \pm}\right)$. (Theorists often prefer the alternate notation of $N_{i}^{0}$ and $C_{i}^{ \pm}$.) The physical masses of these particles, and how strongly they couple to other particles in the theory, are determined by the relative contributions of the pure SUSY states and are highly model dependent.

The implications of supersymmetry for experimental particle physics are enormous. The theory effectively doubles the number of fundamental particles and predicts the existence of a degenerate mass state corresponding to every known Standard Model particle. Immediately one must conclude that SUSY cannot be an exact symmetry; otherwise, we would have already discovered signs of it. For example, the spin-0 selectron, with mass equal to the electron, should be easily produced at current accelerators. Even if direct observation of $\tilde{e}$ were somehow precluded by an unforeseen mechanism, anomalous effects would have been detected in precision electroweak measurements of the Standard Model. 
Fortunately, even if supersymmetry is broken, the theory is not. In theories with "soft" SUSY breaking, supersymmetry is preserved above a mass scale defined by $m_{\text {soft }}$. The quadratically divergent corrections to the Higgs mass continue to cancel out at all orders, and the terms which are logarithmic in $\Lambda_{\mathrm{UV}}$ disappear in the limit of $m_{\text {soft }} \rightarrow 0$. Although the mass degeneracy is lost, these theories predict that $m_{\text {soft }}$ should be on the order of a $\mathrm{TeV}$ or less, which implies that the masses of at least a few of the lightest superpartners could be accessible at current or next-generation accelerators.

In the Standard Model, the number of parameters which must be measured experimentally is 21: twelve quark and lepton masses, three coupling constants, three angles and one phase which describe quark mixing, and two constants associated with the Higgs field. Supersymmetry, in its most general form, introduces over 100 additional parameters. Several models attempt to reduce this complexity, including the popular Minimal Supersymmetry Standard Model (MSSM) [6] and supergravity inspired models (SUGRA) [7]. Rather than constrain the discussion below to any particular version, we shall study the phenomenological consequences of a general form of Supersymmetry.

\subsection{Gluino production and decay}

Despite the number of new parameters (masses, mixing angles, coupling constants, etc.) introduced by the theory, a few are calculable from measured Standard Model quantities due to constraints on the structural form SUSY can take. For instance, the coupling of a gluino to quarks and gluons is related to the strong coupling constant, $\alpha_{s}$. Although the sparticle masses are unknown, one can identify all of the Feynman diagrams which contribute to gluino production at a hadron collider and calculate the cross section for a proposed set of gluino and squark masses. The two lowest order diagrams for gluino pair production are shown in Figure 2.4. The cross section for $\tilde{g} \tilde{g}$ production at the Tevatron is calculated to be on the order of $1 p b$ for particular choices of gluino and squark masses, which is encouraging for the prospect of observing gluino production during Run I at the Tevatron. Further discussion of the production 


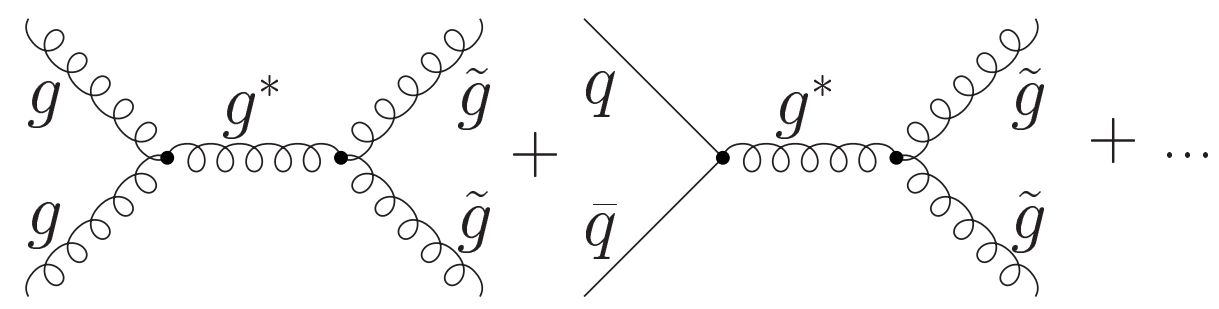

Figure 2.4: At lowest order, gluino pair production follows from an excited gluon state produced by gluon fusion or quark-antiquark annihilation. Higher order Feynman diagrams also contribute significantly to the cross section.

mechanism and cross section appear later in Section 6.2.

Because the gluino carries a net color charge and interacts solely through the strong force, the only allowed decay modes involve quarks and squarks:

$$
\tilde{g} \rightarrow q \tilde{q}_{L, R} \quad \text { or } \quad \tilde{g} \rightarrow \bar{q} \overline{\tilde{q}}_{L, R}
$$

The gluino is a Majorana particle, and the branching ratio for either decay should be $50 \%$ [8]. (A Majorana fermion is one which is equal to its charge conjugate: $\psi^{c}=\psi$. The most familiar example is the Majorana neutrino.)

The squark masses are (nearly) degenerate in many supersymmetric theories. However, the third generation squark masses receive corrections involving the top quark such that $\tilde{t}_{L}$ and $\tilde{t}_{R}$ can be significantly lighter than the first- and secondgeneration squarks. With the additional mixing of $\tilde{t}_{L}$ and $\tilde{t}_{R}$ into the mass eigenstates $\tilde{t}_{1}$ and $\tilde{t}_{2}$, the mass of the lightest stop $\left(\tilde{t}_{1}\right)$ is reduced even further. Therefore, the only kinematically allowed decay mode is $\tilde{g} \rightarrow t \tilde{t}$. Even in a scenario where $m_{\tilde{q}}<m_{\tilde{g}}$, the top-stop mode will dominate over the allowed decays involving other squarks.

The preferred stop decay mode is determined by the relative chargino, neutralino, and sneutrino masses, all of which are model dependent. It is generally believed, though, that the lightest neutralino is the only supersymmetric particle accessible to stop decay. If we assume the mass hierarchy $m_{\tilde{\chi}_{1}^{0}}<m_{\tilde{t}}<m_{\tilde{\chi}_{1}^{+}}, m_{\tilde{\nu}}$, the stop decays via $\tilde{t} \rightarrow c \tilde{\chi}_{1}^{0}$. 


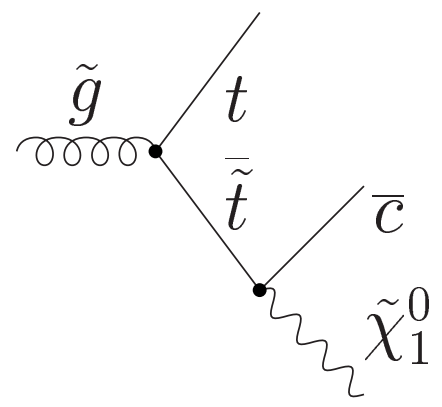

Figure 2.5: Feynman diagram showing the gluino decay to top and scalar top and the subsequent decay of the stop to a charm quark and neutralino.

Therefore, a strong experimental signature of Supersymmetry involves gluino pair production with gluino decay to $t \tilde{t}$, as shown in Figure 2.5. This process provides an alternate mechanism beyond the Standard Model for producing events with top quark pairs. Any excess above the Standard Model prediction for $t \bar{t}$ production could be interpreted as evidence for Supersymmetry [10]. Current limits on the gluino and top mass depend upon the search channel, and a comprehensive summary of the latest results are available elsewhere [9].

The Majorana nature of the gluino brings an interesting twist to the event signature. The equal likelihood of producing top or antitop leads to half of $\tilde{g} \tilde{g}$ events containing either $t t$ or $\overline{t t}$. No processes with this signature exist in the Standard Model, and the discovery of like-sign top events would be unmitigated confirmation of "new physics."

\section{$2.7 \quad$ Top decay}

The top quark, finally discovered in 1994, is unique among the quarks. With a mass in the vicinity of $174 \mathrm{GeV}$, it is more than 30 times heavier than the bottom quark. The top lifetime is fleetingly short: $\tau_{t} \sim 4 \times 10^{-25} \mathrm{~s}$. Whereas other quarks form mesons or baryons after production, the typical timescale for hadronization is a 


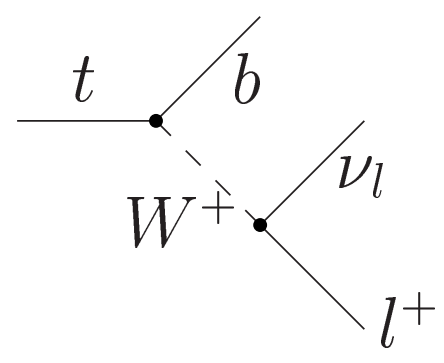

Figure 2.6: Feynman diagram for the leptonic decay of the top quark.

factor of 10 longer than the top lifetime. Thus, top behaves more like a free quark.

The top quark decays predominantly via $t \longrightarrow b W^{+}$. The Standard Model predicts a branching fraction close to unity, and flavor-changing neutral current (FCNC) decays such as $t \rightarrow c \gamma$ and $t \rightarrow c Z^{0}$ are highly suppressed. Experimental limits have been placed on the branching ratio to other decay modes at $<1 \%$.

The $W^{+}$couples to both quarks and leptons and exhibits several hadronic and leptonic decay modes:

$$
\begin{array}{ll}
W^{+} \longrightarrow u \bar{d} & W^{+} \longrightarrow e \overline{\nu_{e}} \\
W^{+} \longrightarrow c \bar{s} & W^{+} \longrightarrow \mu \overline{\nu_{\mu}} \\
& W^{+} \longrightarrow \tau \overline{\nu_{\tau}}
\end{array}
$$

In the absence of color factors for the quarks, the branching ratio for each available mode would be equal. Instead, the hadronic branching ratios receive a multiplicative factor of 3 , and the branching fractions become $\frac{1}{3}$ for each hadronic mode and $\frac{1}{9}$ for each leptonic mode (shown in Figure 2.6).

The Standard Model cross section for $t \bar{t}$ production at the Tevatron is approximately 5-6ph, which roughly translates to the creation of 500 to 600 top pairs during Run I. The signature for top events at CDF varies according to the decay mode of the $W$ bosons. Each $b$ quark hadronizes and produces a jet, while each $W$ provides either additional quarks or leptons. The possible final states have been categorized by CDF as: 
- All-hadronic $\left(\mathrm{BR}=\frac{4}{9}\right)$ : Both $W$ bosons decay hadronically, producing 6 quarks in the final state. This signature is difficult to distinguish from QCD jet production, which has a much larger cross section than $t \bar{t}$. Tagging algorithms have been developed for separating out jets that originate from $b$ quarks. Recent analyses have successfully improved the signal to background ratio in this channel by requiring the identification of one or both of the $b$ jets $[11,12]$.

- Semileptonic $\left(\mathrm{BR}=\frac{4}{9}\right)$ : One $W$ decays hadronically while the other decays leptonically. The final state contains a lepton and 4 jets. The presence of a high-momentum lepton and an energy imbalance due to energy carried away by the neutrino allow for easier triggering on the event. The Lepton + Jets analysis studied this mode by requiring a lepton and missing energy, plus three or more jets, where at least one is $b$-tagged [13].

- Dilepton $\left(\mathrm{BR}=\frac{1}{9}\right)$ : Both $W$ bosons decay leptonically. This final state is the cleanest of the three but suffers from the smallest branching ratio. Dilepton events are characterized by two high-momentum leptons and a large amount of missing energy from the neutrinos. Two jets are insisted upon, but no b-tagging is required.

Events involving the decay of $W$ to tau are ordinarily excluded from consideration in top analyses. The tau displays hadronic and leptonic decay modes which are difficult to distinguish from background sources, although attempts have been made to incorporate the tau channels into the top dilepton analysis [14]. If we ignore the contribution of the tau channels, the effective branching ratios for the lepton + jets and dilepton signatures are reduced to $29.6 \%$ (24/81) and 4.9\% (4/81). All possible $t \bar{t}$ decay modes are summarized in Table 2.3.

\subsection{Search signature for SUSY}

If one wishes to search for gluino production using top events, there are advantages and disadvantages associated with each of the top decay channels. The all-hadronic 


\begin{tabular}{|c|c|c|c|}
\hline$t \bar{t}$ signature & Decay products & \multicolumn{2}{|c|}{ Branching ratios } \\
\hline All-hadronic & $t \bar{t} \rightarrow \quad b \bar{b} q \bar{q} q \bar{q}$ & $36 / 81$ & $36 / 81(44.4 \%)$ \\
\hline \multirow[t]{2}{*}{ Lepton + jets } & $\begin{array}{l}t \bar{t} \rightarrow \quad b \bar{b} q \bar{q} e \nu \\
t \bar{t} \rightarrow \quad b \bar{b} q \bar{q} \mu \nu\end{array}$ & $\begin{array}{l}12 / 81 \\
12 / 81\end{array}$ & $24 / 81(29.6 \%)$ \\
\hline & $t \bar{t} \rightarrow \quad b \bar{b} q \bar{q} \tau \nu$ & $12 / 81$ & \\
\hline \multirow{2}{*}{ Dilepton } & $\begin{array}{l}t \bar{t} \rightarrow \quad b \bar{b} e^{+} e^{-} \nu \bar{\nu} \\
t \bar{t} \rightarrow \quad b \bar{b} \mu^{+} \mu^{-} \nu \bar{\nu} \\
t \bar{t} \rightarrow \quad b \bar{b} e^{ \pm} \mu^{\mp} \nu \bar{\nu}\end{array}$ & $\begin{array}{l}1 / 81 \\
1 / 81 \\
2 / 81\end{array}$ & $4 / 81(4.9 \%)$ \\
\hline & $\begin{array}{ll}t \bar{t} \rightarrow & b \bar{b} e^{ \pm} \tau^{\mp} \nu \bar{\nu} \\
t \bar{t} \rightarrow & b \bar{b} \mu^{ \pm} \tau^{\mp} \nu \bar{\nu} \\
t \bar{t} \rightarrow & b \bar{b} \tau^{+} \tau^{-} \nu \bar{\nu}\end{array}$ & $\begin{array}{l}2 / 81 \\
2 / 81 \\
1 / 81\end{array}$ & \\
\hline
\end{tabular}

Table 2.3: All available decay modes from top pair production and their branching ratios. The modes involving tau are considered separately in top analyses, so the branching fractions for channels involving only electrons and muons are also shown. 
mode and lepton + jets modes offer larger branching fractions, but a $\tilde{g} \tilde{g}$ signal competes against the existing $t \bar{t}$ production. The signal to background ratio is potentially small, and evidence of SUSY would have to come from an observed excess of events compared to the Standard Model. To prove a discrepancy with the SM prediction would require a firm understanding of the backgrounds and the production cross sections, as well as a larger data sample than collected during Run I.

The dilepton mode offers a smaller background but suffers from a much lower branching ratio. The same difficulties with the cross section and sample size would make unlikely the ability to claim any excess in the top dilepton channel is due to gluino events. On the other hand, the equal likelihood of gluino pair production leading to like-sign top events becomes extremely useful here. Whereas any information about the top charges is lost in the all-hadronic and lepton + jets channels, the dilepton mode benefits through the appearance of like-sign leptons in the final state. The Standard Model offers very few processes which create a same-sign dilepton signature, so the backgrounds are extremely small. A strong potential exists for observing or setting limits on gluino production in the like-sign top dilepton mode, and this is the motivation for the search described here.

In the next chapter, we explain how high energy physics events are created at Fermilab using the Tevatron accelerator. A description of the CDF experimental apparatus follows, and we detail the detector systems which are particularly important in identifying the various elements (electrons, muons, jets) which constitute the likesign top dilepton signature. 


\section{Chapter 3}

\section{The CDF Experiment}

\subsection{The Tevatron}

Fermi National Accelerator Laboratory is located outside Batavia, IL, about 40 miles west of Chicago. Fermilab is currently home to the highest energy particle accelerator in the world. The Tevatron is a circular accelerator with a radius of $1 \mathrm{~km}$ (0.6 mi) and circumference of $6.3 \mathrm{~km}(3.9 \mathrm{mi})$. Two beams of particles traverse the ring in opposite directions and, once they have been accelerated to sufficient energy, are collided head-on. The proton beam travels clockwise around the ring, while the antiproton beam circles counterclockwise. Their trajectories actually lie inside the same beampipe but form helical paths, so that there is no interaction between them while the energy is being ramped up. Once the maximum energy has been attained and collisions are desired, the intersection point can be carefully controlled and positioned at very specific locations on the ring called interaction regions. There are five such locations at the Tevatron; two of these are occupied by the CDF (Collider Detector at Fermilab) and D0 (D-Zero) experiments.

This analysis is based on data collected by CDF during Run I of the Tevatron from 1992 to 1996. The initial run, known as Run Ia, lasted from August 1992 to July 1993. After a brief hiatus, Run Ib began in January 1994 and continued until July 1995. Later that year, a brief run was added, which we mention for completeness although we do not make use of data collected during Run Ic, just before the Tevatron was 
switched off in early 1996. After a long period of accelerator and detector upgrades, Run II of the Tevatron began last year, in March 2001.

\subsubsection{Basic accelerator physics}

The energy of the particle beams is measured in terms of the electron-volt $(\mathrm{eV})$, which is the amount of energy an electron gains by being accelerated across one volt of potential difference. This is in fact a tiny amount of energy:

$$
1 \mathrm{eV}=1.6 \times 10^{-19} \mathrm{~J}=3.8 \times 10^{-20} \mathrm{cal}
$$

During Run I at the Tevatron, each beam had an energy of $900 \mathrm{GeV}$ (or 9 billion electron-volts). The particles in the two beams traveled at $99.99995 \%$ of the speed of light, or just $365 \mathrm{mph}$ less than the speed of light (671.5 million mph, or 186500 $\mathrm{mi} / \mathrm{s})$.

When a particle and antiparticle interact, they mutually annihilate, and the resulting energy is available for new particle production. The advantage of a colliding beam experiment versus a fixed target one is the increased center of mass energy $\left(E_{c m}\right.$ or $\sqrt{s}$ ). An antiproton with $v=\frac{1}{3} c$ interacting with a proton traveling in the opposite direction with $v=\frac{1}{3} c$ is vastly different from the situation where an antiproton with $v=\frac{2}{3} c$ smashes into a stationary proton. In the fixed target case, the center of mass energy scales as $\propto E_{\text {incident }}^{1 / 2}$, while for colliding beams of equal energy, the energy scales as $\propto E_{\text {beam }}$. Designing an experiment to collide two extremely small beams is technically more difficult than directing a single beam onto a target, but the increased energy available to produce new particles makes the effort worthwhile.

The probability of a single proton interacting with a single antiproton is extremely small. Therefore, a significant number of particles must be collected for each beam, and the numbers are chosen such that the average number of interactions is approximately 1-2 for each crossing of the beams.

The event rate $R$ (number of events per unit time) is determined by two factors, one dependent upon the physical parameters of the collider and the other inherently 
dependent upon the nature of the interacting particles:

$$
R=\mathcal{L} \sigma_{\text {int }}
$$

The cross section, $\sigma_{i n t}$, is a measure of the total number of ways in which the two particles may interact. In a manner of speaking, it is the effective target size that one particle presents to the other, and as such is expressed in units of cross-sectional area. Because the sizes involved are so tiny, $\sigma$ is usually given in units of barns:

$$
1 \mathrm{~b}=10^{-24} \mathrm{~cm}^{2}=\left(10^{-14} \mathrm{~m}\right)^{2}
$$

During Run I, the total cross section for $p \bar{p}$ collisions at $\sqrt{s}=1800 \mathrm{GeV}$ was measured to be $\sigma_{\text {total }}=80.03 \pm 2.24 \mathrm{mb}$ [15], which includes all elastic and inelastic interactions. Many processes that we are interested in have cross sections on the order of a picobarn $\left(1 \mathrm{pb}=10^{-36} \mathrm{~cm}^{2}\right)$.

The luminosity $\mathcal{L}$ describes the number of events per unit cross section for each interaction of the two beams, per unit time $\left(\mathrm{cm}^{-2} \mathrm{~s}^{-1}\right)$. For simplicity, consider the case where each beam consists of a single bunch of particles. Let the number of protons in a bunch be $N_{1}$ and the number of antiprotons per bunch be $N_{2}$, and let $f$ be the frequency of intersection of the bunches. Then the number of possible intersections per unit time is $f N_{1} N_{2}$. However, the particles involved are extremely small and are spread out over an area $A$, similar to a finely misted cloud. So the number of interactions per unit area per unit time is given by:

$$
\mathcal{L}=n \frac{f N_{1} N_{2}}{A}=n \frac{f N_{1} N_{2}}{4 \pi \sigma^{2}}
$$

where a multiplicative factor $n$ accounts for the case where each beam is composed of $n$ bunches instead of just one. The area $A$ is often expressed in terms of the Gaussian spread of the beam $\sigma$ in the horizontal and vertical directions perpendicular to the beam momentum.

During Run I, the Tevatron ran with $6 p$ bunches on $6 \bar{p}$ bunches which were equally spaced. The revolution frequency $f$ was about $50 \mathrm{kHz}$, which translates to a $3.5 \mu$ s interval between bunch crossings. The number of protons per bunch was typically $N_{1}=2 \times 10^{11}$ and the number of antiprotons was $N_{2}=6-7 \times 10^{10}$. The 
beams were spread out over a diameter of $\sigma=70 \mu \mathrm{m}$, so $\sigma^{2}=5 \times 10^{-5} \mathrm{~cm}^{2}$. During Run Ia, a typical and highest initial instantaneous luminosity were $0.54 \times 10^{31}$ and $0.92 \times 10^{31} \mathrm{~cm}^{2} \mathrm{~s}^{-1}$. During Run Ib, they were $1.6 \times 10^{31}$ and $2.8 \times 10^{31} \mathrm{~cm}^{2} \mathrm{~s}^{-1}$.

\subsubsection{Creating the beams}

The Tevatron design is shown in Figure 3.1. The protons originate from $\mathrm{H}_{2}$ gas which is ionized to produce $\mathrm{H}^{-}$. A Cockcroft-Walton accelerator accelerates them up to $750 \mathrm{keV}$ and passes them to a 150-meter linear accelerator which ramps the energy up to $200 \mathrm{MeV}$. The ions are then passed through a thin carbon foil target which strips off the electrons, leaving the proton (hydrogen nucleus). The Booster ring $($ circumference $=475 \mathrm{~m}$ ) accelerates the particles up to $8 \mathrm{GeV}$ before inserting several bunches into the Main Ring, the predecessor of the Tevatron. The Main Ring and Tevatron occupy the same 6300-meter (circumference) tunnel, with the Tevatron beamline and magnets mounted directly below the original $400 \mathrm{GeV}$ accelerator. Once inside the Main Ring, the energy is increased to $150 \mathrm{GeV}$, and the bunches are combined to form a single bunch to be injected into the Tevatron ring. This process is repeated to produce the six Tevatron bunches.

The $\bar{p}$ beams are more difficult to produce because antiprotons are not so readily available. Any naturally occurring antiparticles exist for only a short time before encountering ordinary particles and disappearing in a burst of energy, so they must be manufactured and stored until a sufficient number are available for collisions. Protons are taken from the Main Ring and directed onto a heavy nucleus target, tungsten in the case of the Tevatron. The interaction results in proton-antiproton production, and the $\bar{p}$ are collected and focused using a lithium "lens". The resulting antiprotons from such an energetic, coarse process suffer from a large momentum spread due to random motions, and they must be stochastically "cooled" in the Antiproton Debuncher. Here a more stable electron beam interacts with the antiprotons and carries off some of the random momenta, thereby reducing the $\bar{p}$ temperature. Then the antiprotons are stored in the Accumulator until enough are ready for injection into the Main Ring and, subsequently, the Tevatron. This process requires on the order of a few hours 


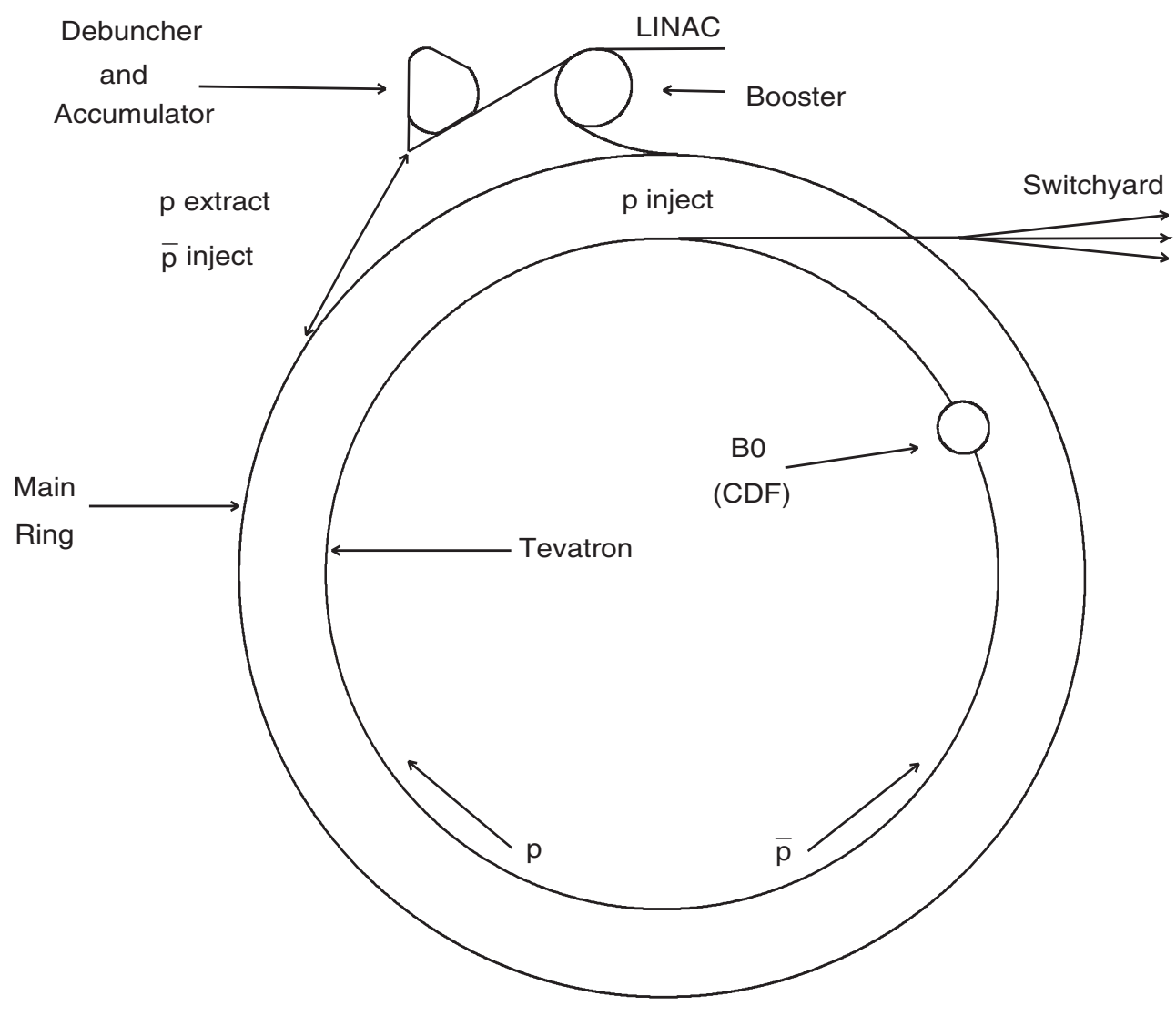

Figure 3.1: Layout showing the Fermilab accelerator stages leading up to injection of the beams into the Tevatron. 
up to half a day.

\subsection{Collider Detector at Fermilab (CDF)}

One quadrant of the CDF detector [17] is shown in Figure 3.2. Standing outside the collision hall in the staging area, the coordinate system is as shown in the upperleft hand section of the diagram. The $x$-axis points radially outward from the center of the Tevatron ring and out of the page. The $y$-axis is directed upward, and the positive $z$-axis is to the left, in the direction of proton travel through CDF. The detector is symmetric in azimuthal angle $\phi$ (in the $x-y$ plane), so cylindrical coordinates are preferred: radius $r$, angle $\phi$, and $z$. Alternately, the polar angle $\theta$ is measured with respect to the positive $z$-axis.

\subsubsection{Some definitions}

In particle physics, it is convenient to work in terms of rapidity rather than $\theta$. The rapidity of a particle is defined as:

$$
y \equiv \frac{1}{2} \ln \left(\frac{E+p_{z}}{E-p_{z}}\right)=\tanh \left(\frac{p_{z}}{E}\right)
$$

where $p_{z}$ is the $z$-component of the momentum and the energy $E$ is $\sqrt{p^{2} c^{2}+m^{2} c^{4}}$. The advantage here is that if we change our frame of reference to that of a particle travelling along the $z$-axis with velocity $\beta=v / c$, the rapidity transforms as $y \rightarrow$ $y-\tanh \beta$. Any distribution of events in $y, d N / d y$, changes only by a constant term, and the shape remains unchanged.

The disadvantage is that the rapidity depends upon the mass of the particle involved, which is not necessarily measurable for a given object seen in the detector. In the case where the momentum is much larger than the mass $(p \gg m)$, the rapidity can be approximated as [16]:

$$
y \equiv \frac{1}{2} \ln \frac{\cos ^{2}(\theta / 2)+m^{2} / 4 p^{2}+\cdots}{\cos ^{2}(\theta / 2)+m^{2} / 4 p^{2}+\cdots} \approx-\ln \tan \left(\frac{\theta}{2}\right) \equiv \eta
$$




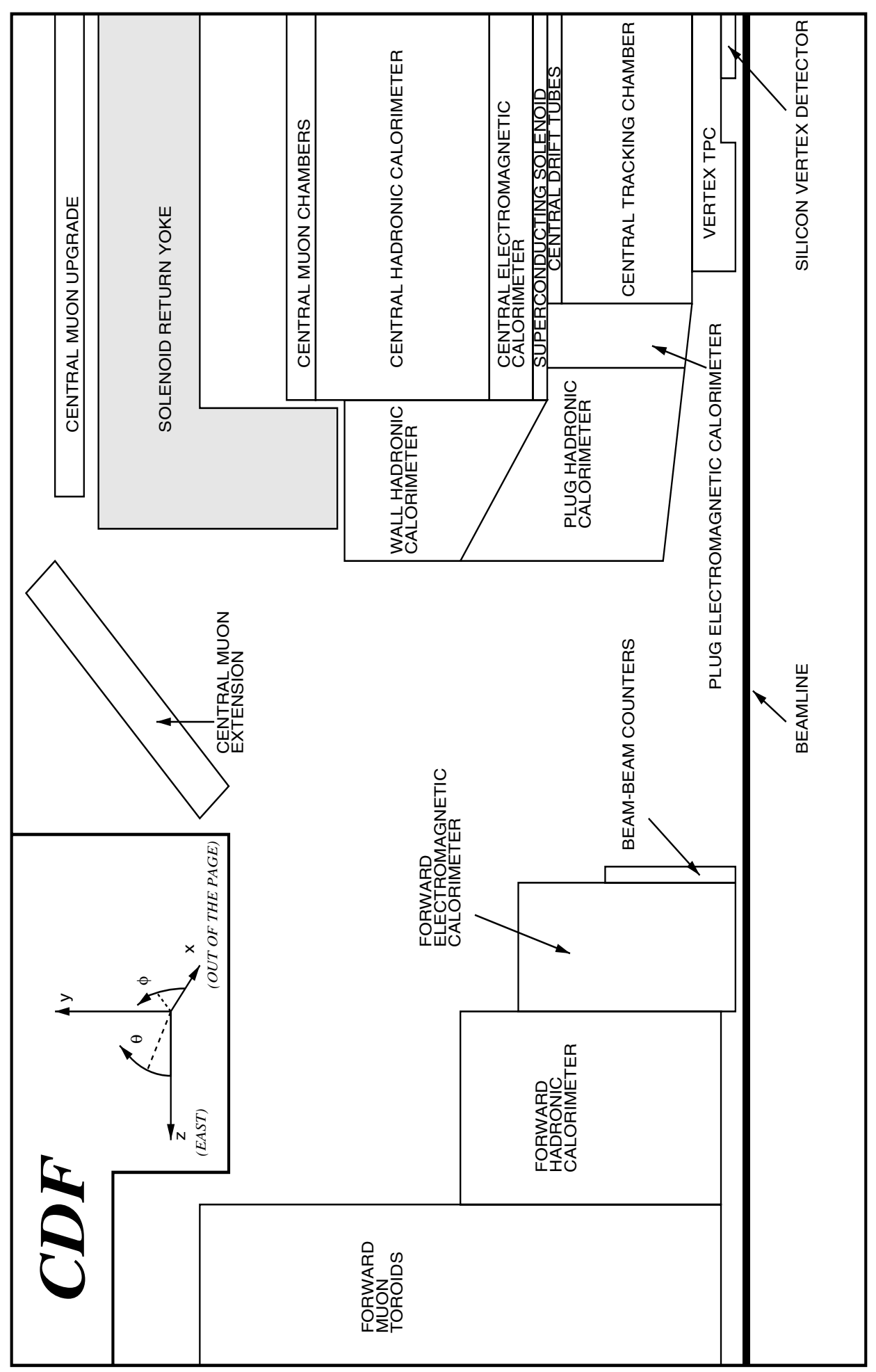

Figure 3.2: Single quadrant schematic of the CDF detector. 
where $\cos \theta=p_{z} / p$. The pseudorapidity $\eta$ is determined solely by measuring the polar angle $\theta$ and does not require knowledge of the particle type or momentum. For the detector components described below, the angular coverage will be discussed in terms of $\eta$ rather than $\theta$.

Another important concept is the idea of transverse momentum and energy. In the collision between a proton and an antiproton, the constituent quarks and gluons are what actually interact with each other. Although we know the overall beam energy and momentum, we cannot predict what fraction is carried by each constituent (or parton). The initial momentum along the beamline is completely unknown, so the usefulness of $p_{z}$ information after the collision is diminished. One thing we can say is that the total momentum perpendicular to the beam axis should be relatively tiny. Conservation dictates that for the final state particles the sum of momenta in the transverse direction will also be near zero.

The momenta and energies of the outgoing particles are measured using the central tracking detectors and calorimetry systems, respectively. (These will be described in more detail shortly.) We then define the transverse momentum and transverse energy as:

$$
\begin{aligned}
& p_{T}=p \sin \theta \\
& E_{T}=E \sin \theta
\end{aligned}
$$

Energy is not a vector quantity but is treated as such here because each particle is directed at and deposits its energy in a very specific detector location. Measurement of energy in the calorimeter is essentially a measure of the particle momentum for high $p_{T}$ particles.

When objects like neutrinos (and neutralinos, assuming they exist) are produced at the Tevatron, they escape the apparatus undetected and can carry away significant momenta and energy. After summing up all of the $p_{T}$ and $E_{T}$ for particles detected in an event, any difference from zero indicates that these particles may have been present. This concept is known as missing transverse energy and is usually referred to as "missing $E_{T}$ " or $\mathbb{E}_{T}$.

The CDF detector is not perfect, though. It is impossible to provide full angular coverage in the central region with active devices because space is needed for cabling, 
support structures, etc. Occasionally particles will escape through gaps between detectors, or individual energy and momenta are mismeasured. Therefore, one must check that the missing energy direction does not point to "cracks" in the detector or line up with known particles in the event before assuming that any missing $E_{T}$ is a result of undetected particles.

\subsubsection{Detector overview}

The CDF detector is characterized by a central tracking volume located within a 5$\mathrm{m}$ long superconducting solenoidal magnet with radius of $1.5 \mathrm{~m}$ and uniform 1.4 Tesla magnetic field. This allows measurement of the charge and momentum of charged particles due to the curvature of their paths in the magnetic field. The direction of curvature indicates the charge, while the radius is related to the momentum through the simple relation

$$
p=0.3 B \rho .
$$

where $p$ is the momentum (in $G e V / c$ ), B is the field strength (in Tesla), and $\rho$ is the track radius (in $m$ ).

In the central region, a silicon microstrip tracker, gas vertex chamber, and wire chamber comprise the tracking system. Outside the magnetic field are electromagnetic and hadronic calorimeters, which provide information on electrons and jet activity. After a layer of shielding, the outer region of the detector is populated by several muon systems. These are described in detail in the following sections.

On each end of the detector is located a Plug region containing more EM and hadronic calorimetry. These endcaps are removable so as to allow access to the central tracking area. Additional instrumentation is placed upstream and downstream, providing further calorimeter and muon coverage in the Forward regions. However, none of the Plug or Forward systems were used in this analysis. 


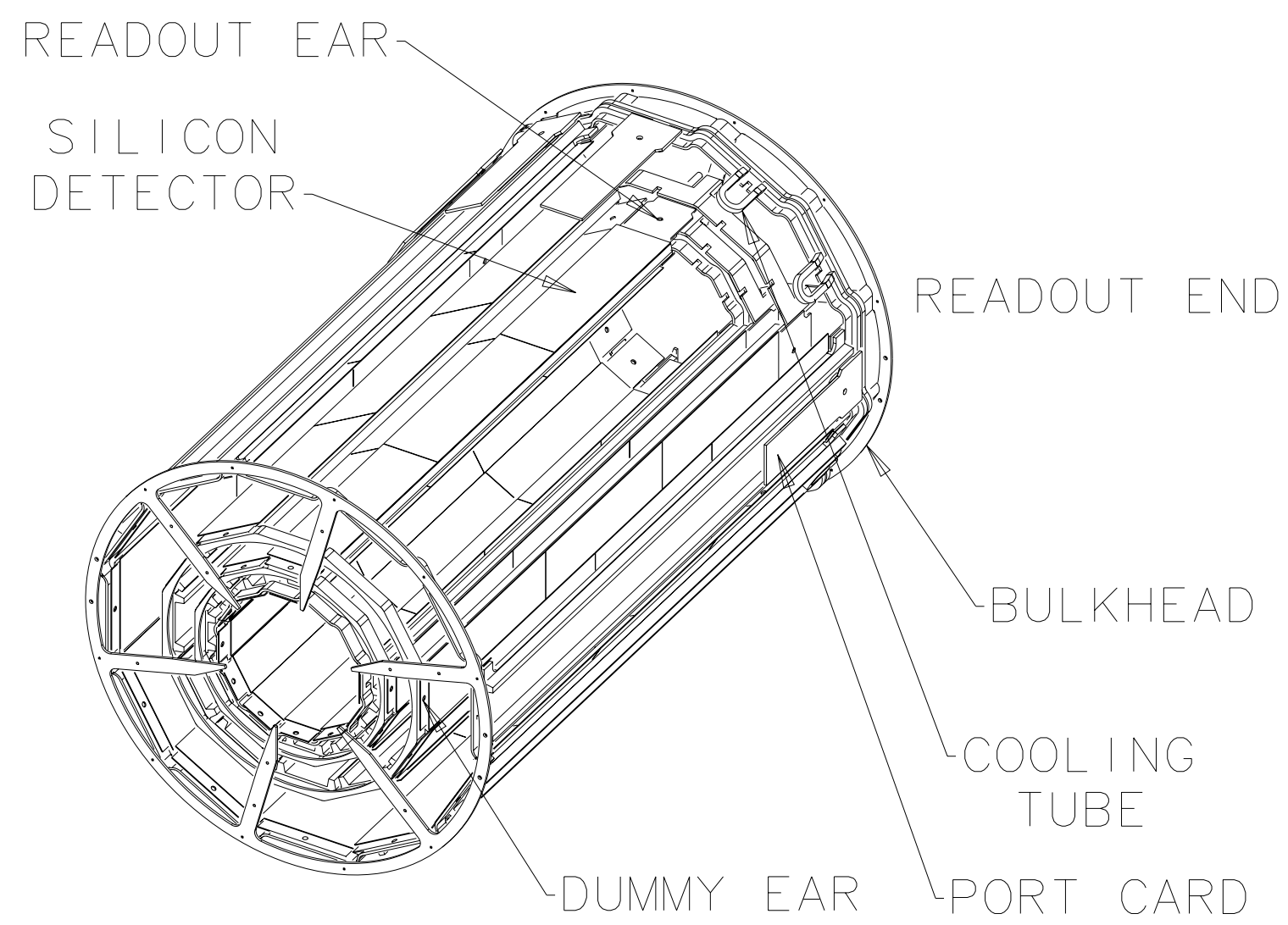

Figure 3.3: Schematic for one of the two identical SVX barrels. Three silicon microstrip detectors are joined to create a ladder, and twelve ladders arranged in $\phi$ at equal radius form a layer. There are four layers, with radii from 2.86 to $7.87 \mathrm{~cm}$. The bulkhead provides structural support as well as channels for cooling the system. Readout of the system is performed at the outer ends.

\subsection{Silicon Vertex Detector (SVX)}

The closest detector to the interaction region, a "Silicon Vertex Detector" in the case of CDF, has the best vantage point to observe the collision products. It should be finely segmented to provide the highest resolution possible and, due to its proximity to the collisions, must be designed with materials that can withstand the high radiation dosage.

One half of the Silicon Vertex Detector (SVX [18]) is shown in Figure 3.3. Two such barrels are placed end-to-end, for a total length of $51 \mathrm{~cm}$, or rapidity coverage 


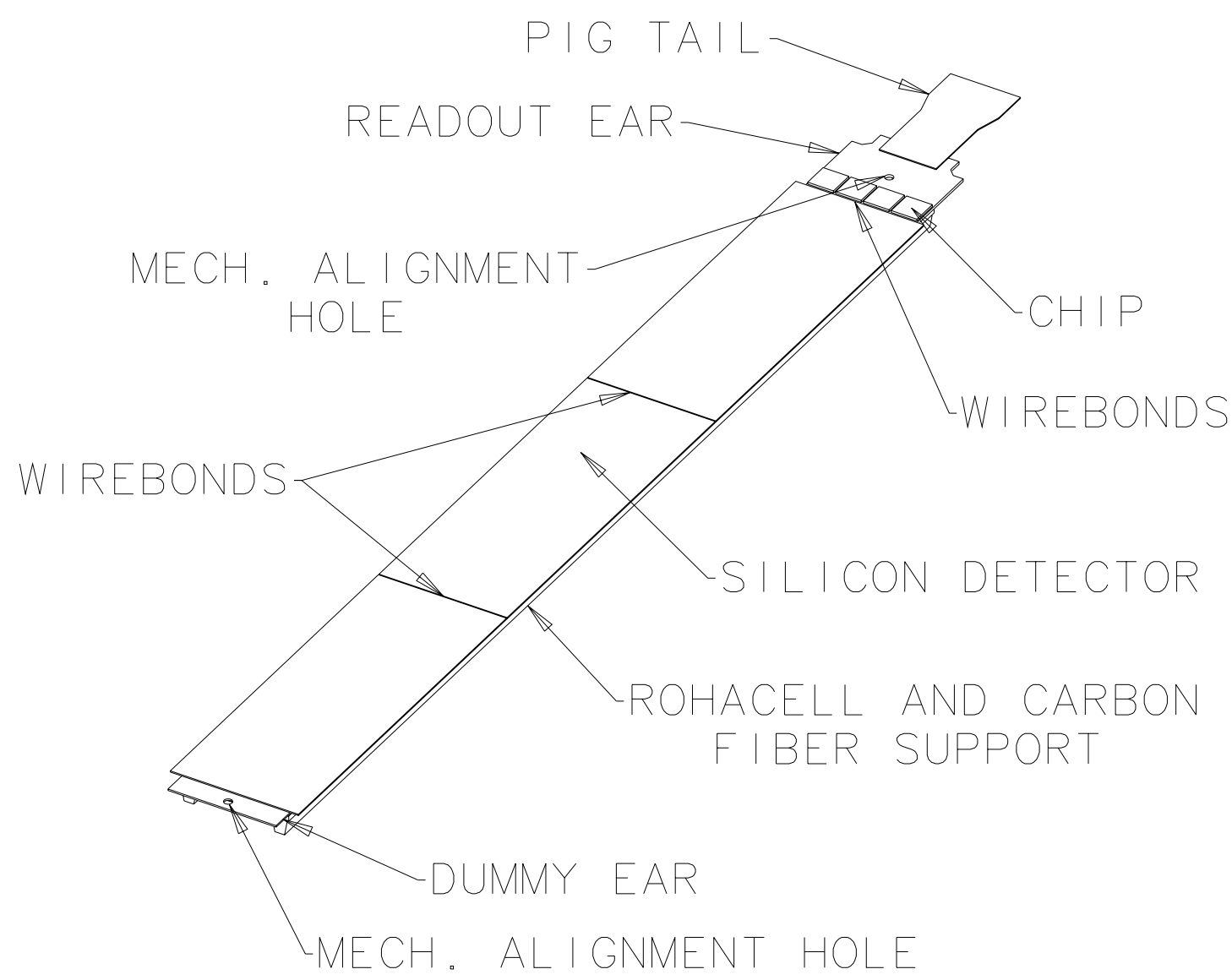

Figure 3.4: Layout of an individual SVX ladder. Three silicon detectors are mounted onto a lightweight Rohacell support and wirebonded to form a single electrical unit, with effective strip lengths of $25 \mathrm{~cm}$. Radiation-hard readout electronics are attached at the far end of the ladder.

of $|\eta|<1.9$. The interaction region is centered at $z=0$ but has a large Gaussian spread of $\sigma=30 \mathrm{~cm}$, which translates to $\sim 60 \%$ geometrical acceptance for outgoing tracks.

The basic unit of the SVX is a silicon microstrip detector. In the SVX design, these were single-sided devices, $8 \mathrm{~cm}$ in length and $2-5 \mathrm{~cm}$ in width, with an active silicon layer of $\sim 300 \mu \mathrm{m}$ thickness. The sensor works as a large $p-n$ junction, and a voltage is applied between the top and bottom surfaces to deplete the bulk of free charge carriers. An array of $10 \mu \mathrm{m}$-wide aluminum strips with $55-60 \mu \mathrm{m}$ pitch run the length of one side (hence, single-sided). Three sensors are mounted in a 
ladder, and the strip ends of adjacent detectors are wirebonded together, effectively creating a single device with $25 \mathrm{~cm}$ strips. When a charged particle passes through the sensor, it ionizes the material, and the released charges drift in the electric field and are collected by the strips. The readout system measures the amount of charge collected per strip, and the distribution of charge indicates where the initial particle passed through, to a precision of $13 \mu \mathrm{m}$.

Twelve ladders are distributed in $\phi$, with some overlap to avoid cracks, to form a layer. Each barrel contains four concentric layers, the innermost at radius $\mathrm{r}=2.68 \mathrm{~cm}$ and outermost at $\mathrm{r}=7.87 \mathrm{~cm}$. In all, there are 46,080 readout channels for the SVX.

All strips are aligned parallel to the beamline. Therefore, they only locate particles in $r$ and $\phi$ with no $z$ information other than the fact that the track passed through a particular ladder. By combining hit information from the four layers of silicon with seed data from detectors at larger radii, particle tracks can be fully reconstructed. The impact parameter resolution for high momentum tracks is $17 \mu \mathrm{m}$.

After Run Ia, the readout electronics of the original SVX had sustained significant radiation damage, leading to increased leakage currents and causing the signal-tonoise ratio to drop from 9 to 6 . A replacement system, SVX' (read "SVX prime") [19], was installed prior to Run Ib. For SVX', the readout chips were made radiation hard, able to withstand the increased luminosity for Run Ib, and electrical changes were made to the sensor design to reduce noise, resulting in an initial $\mathrm{S} / \mathrm{N}$ ratio of 16 . Also, the inner layer was moved to $\mathrm{r}=2.86 \mathrm{~cm}$ and redesigned to close gaps in coverage.

\subsection{Vertex Time Projection Chamber (VTX)}

In order to complement the $r-\phi$ only information from the SVX and the tracking ability of the larger Central Tracking Chamber (CTC) which surrounds it, the Vertex Time Project Chamber (VTX) [20] provides the necessary $r-z$ track information. The event location in $z$ is important for calculating physics quantities, such as transverse energy $E_{T}$, and for aiding the CTC in 3-D track reconstruction. Events may contain over a hundred charged particles, and detailed $r-z$ tracking is necessary for proper reconstruction of the event. It also helps the outer detectors distinguish true electrons 
from photon conversions $\left(\gamma \rightarrow e^{+} e^{-}\right)$. Photons passing through the inner regions of the detector are less likely to have converted because they have passed through less material. The absence of tracks in the VTX that correspond to ones in the CTC would indicate conversion electrons. Finally, the probability of multiple interactions per beam crossing requires that the identification of multiple vertices along $z$ be possible.

The VTX has a radius of $22 \mathrm{~cm}$ and provides tracking coverage out to $|\eta|<3.25$. The detector consists of 8 octagonal modules along the z-axis, and each is divided into 8 wedges in $\phi$. The chambers are filled with argon-ethane gas, and the endcaps are outfitted with sense wires that are perpendicular to the beamline and to a radial line extending from the center. When a charged particle passes through the chamber, the gas is ionized, and the freed electrons drift along the $z$ direction until striking the sense wires on either end. The individual signal wires provide radial information, while the difference in arrival time for the signal on each end of the chamber may be converted into the $z$ position of the original particle trajectory. The resolution of the detector along $z$ is about $1 \mathrm{~mm}$.

\subsection{Central Tracking Chamber (CTC)}

The basic unit of a wire chamber, known as a cell, is shown in Figure 3.5. Two sets of long, fine wires are tightly strung between the ends of the chamber, and the cell is filled with gas, an argon-ethane-ethanol mixture (49.6\%/49.6\%/0.8\%) in the case of CDF. When a charged particle passes through the cell and ionizes the gas, the free electrons drift in the electric field produced by the potential wires and the magnetic field of the toroid. As they drift, the electrons gain energy and cause further ionization, creating a cascade effect. The electron shower continues to develop until the electrons are collected by one or more of the sense wires.

The Central Tracking Chamber (CTC) [21] is a cylindrical chamber 3.2 meters in length and has an inner (outer) radius of 0.31 (1.32) meters. This translates into rapidity coverage of $|\eta|<1.0$. The drift cells described above are arranged into 9 "superlayers", as shown in Figure 3.6. Cells in even layers (0, 2, 4, 6, 8) contain 12 


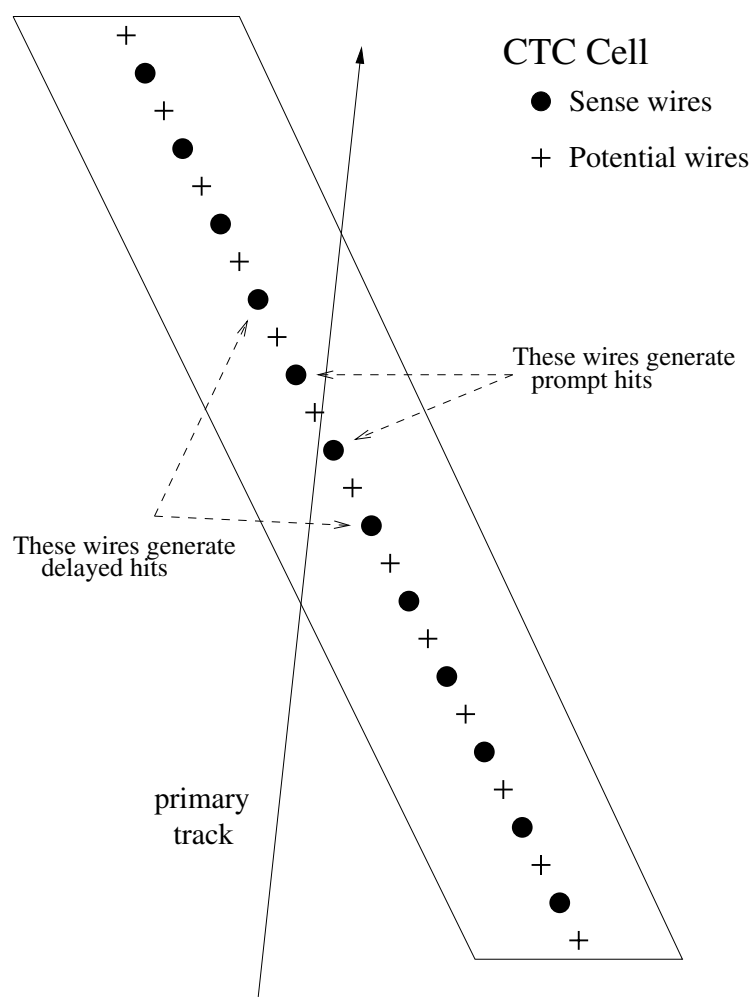

Figure 3.5: An individual cell of the Central Tracker Chamber. As a track passes through and ionizes the gas, the drift electrons are collected by the sense wires, and the charge distribution measures the track position. Potential wires aid in shaping the electric field. 


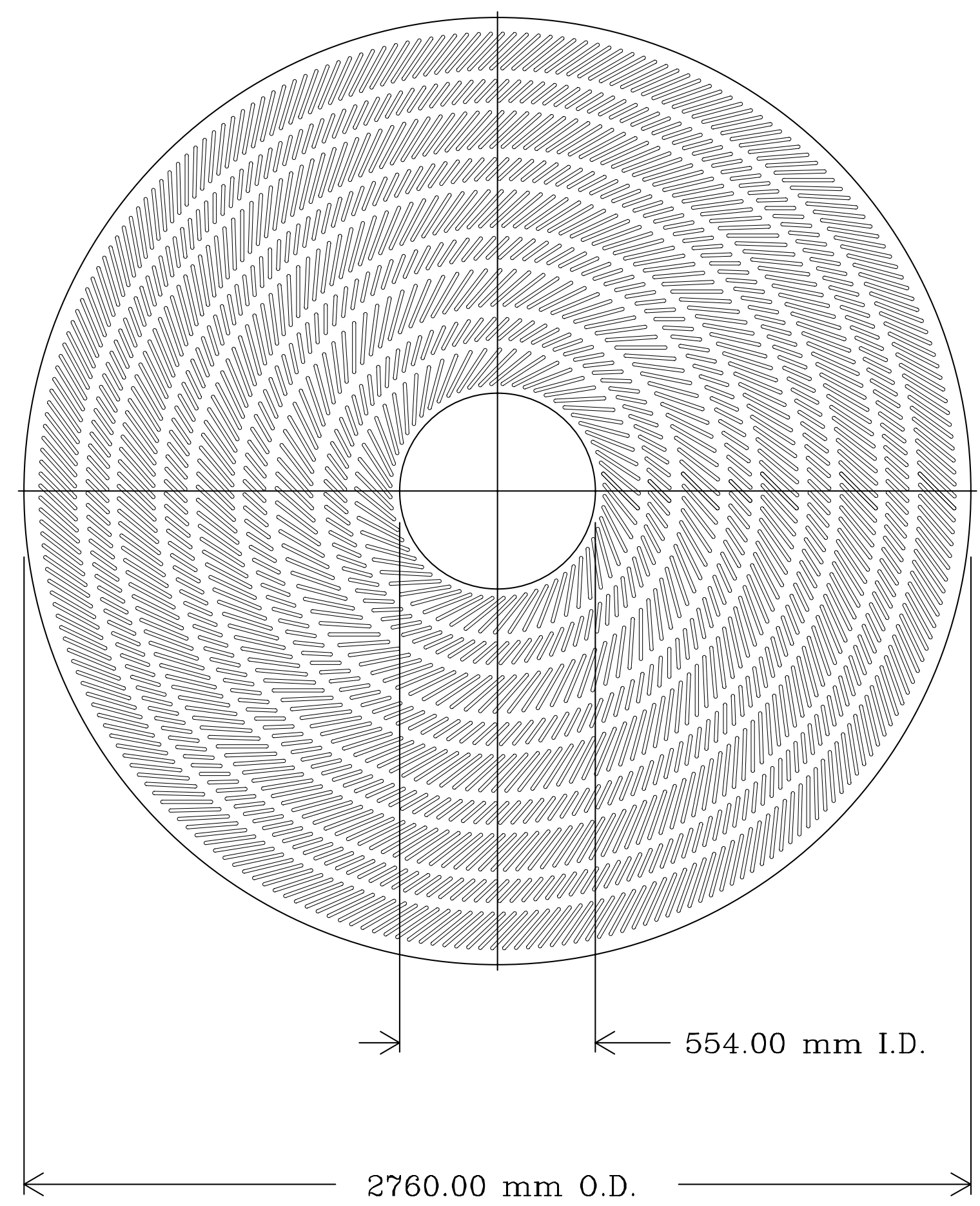

Figure 3.6: End view of the Central Tracking Chamber, showing the 9 superlayers (0-8) and the $45^{\circ}$ tilt of the drift cells with respect to the radial direction. The larger axial cells occupy the even layers, while the smaller stereo cells comprise the odd layers. 
sense wires and in odd layers $(1,3,5,7)$ contain 6 each, creating 84 concentric layers of wires. In the even superlayers, the wires are strung parallel to the central beam axis and provide $r-\phi$ (axial) information. In the odd superlayers, the wires are offset $3^{\circ}\left(+3^{\circ}\right.$ in layers 1 and $5,-3^{\circ}$ in layers 3 and 7$)$ with respect to the beamline and provide $r-z$ (stereo) tracking.

In the $1.4 \mathrm{~T}$ magnetic field and $1350 \mathrm{~V} / \mathrm{cm}$ electric field, the Lorentz angle of the electron drift velocity can be made nearly azimuthal $(\phi)$ by tilting the drift cells $45^{\circ}$ with respect to the radial direction. The overlapping of wires in adjacent cells guarantees that a radial, large momentum track must pass close to at least one wire in each superlayer. This fact is exploited in the event trigger for high- $p_{T}$ tracks.

The hit resolution for each wire is about $200 \mu \mathrm{m}$, and for closely spaced tracks, the two-track resolution is around $5 \mathrm{~mm}$. For high- $p_{T}$ tracks, the momentum resolution is $\delta p_{T} / p_{T} \approx(0.2 \%) \times p_{T}$, where the momentum is given in $G e V / c$. When tracking information from the CTC is projected back into the SVX and combined with hits in the silicon, the overall tracking resolution is $\delta p_{T} / p_{T} \approx(0.1 \%) \times p_{T}$.

\subsection{The CDF Calorimeters}

Particles with transverse momentum $p_{T}<350 \mathrm{MeV}$ become trapped within the magnetic field of the tracking region, while more energetic particles escape and enter the calorimetry. Whereas the tracking chambers measure a trajectory with relatively small energy loss to the particle as it ionizes the material, calorimeters are designed to absorb a large fraction of the incident energy. As shown in Figure 3.2 earlier, the calorimeter consists of components in the Central, Plug, and Forward regions, with each having electromagnetic and hadronic subsystems. We are concerned only with the central detectors: the Central Electromagnetic Calorimeter (CEM) [22] and the Central Hadronic (CHA) and Wall Hadronic Calorimeters (WHA) [23]. For completeness, some information for the other systems is given in Table 3.1.

The CEM starts at $r=173 \mathrm{~cm}$ and is $35 \mathrm{~cm}$ thick, and the CHA extends another $133 \mathrm{~cm}$ beyond that. Both detectors are divided azimuthally into 24 wedges, each subtending an angle of $15^{\circ}$, and they extend out to $z= \pm 250 \mathrm{~cm}$. The resulting 


\begin{tabular}{|c|c|c|c|}
\hline \hline System & Pseudorapidity & $\Delta \eta \times \Delta \phi$ & $N_{\text {layers }}$ \\
\hline CEM & $|\eta|<1.1$ & $0.11 \times 15^{\circ}$ & 30 \\
CHA & $|\eta|<0.9$ & $0.11 \times 15^{\circ}$ & 32 \\
WHA & $0.7<|\eta|<1.3$ & $0.11 \times 15^{\circ}$ & 15 \\
\hline PEM & $1.1<|\eta|<2.4$ & $0.09 \times 5^{\circ}$ & 34 \\
PHA & $1.3<|\eta|<2.4$ & $0.09 \times 5^{\circ}$ & 20 \\
\hline FEM & $2.2<|\eta|<4.2$ & $0.10 \times 5^{\circ}$ & 30 \\
FHA & $2.4<|\eta|<4.2$ & $0.10 \times 5^{\circ}$ & 12 \\
\hline \hline
\end{tabular}

Table 3.1: Summary of pseudorapidity coverage and angular segmentation for all Central, Plug, and Forward electromagnetic and hadronic calorimeters.

pseudorapidity coverage is $|\eta|<1.1$ and $|\eta|<0.9$, respectively. The layers of material are segmented into portions of $\Delta \eta=0.11$, creating towers that project back to the interaction region. This structure is displayed from a couple different perspectives in Figure 3.7.

\subsubsection{Central electromagnetic calorimeter (CEM)}

An EM calorimeter is designed to measure the energy of electrons and photons using the phenomena of bremsstrahlung and pair production. In the first case, electrons enter the dense absorbing material and slow down, and the (de)acceleration causes radiation of photons. This is true of any charged particle undergoing a change in speed or direction, but electrons are particularly susceptible because of their small mass. In the second case, photons traverse the material and interact, causing the creation of electron-positron pairs. For an initial high-energy $e$ or $\gamma$, the secondary, tertiary, etc., electrons and photons will be energetic enough to trigger these processes as well, giving rise to a cascade effect. Thin slices of scintillating material, which give 


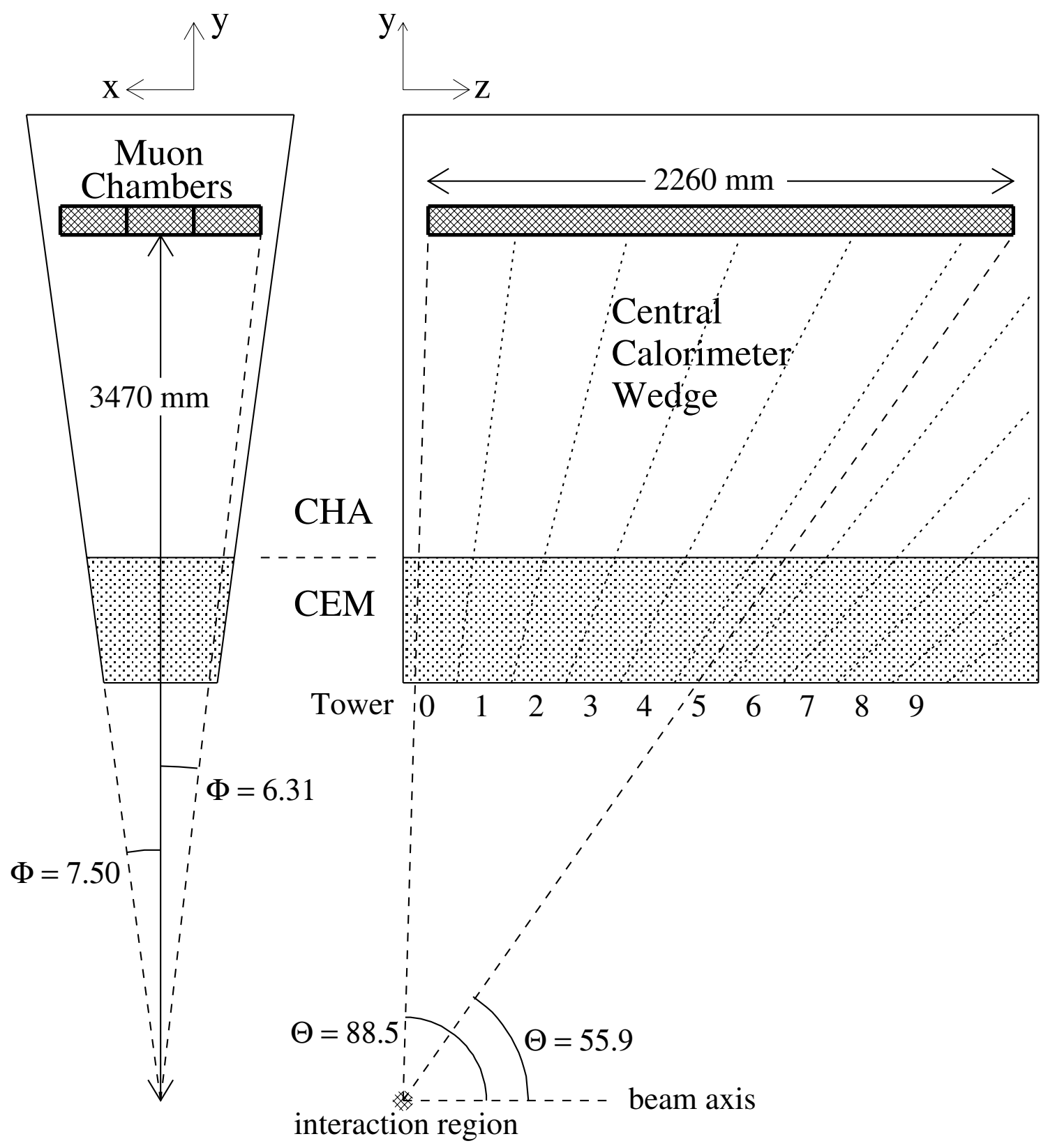

Figure 3.7: Two images showing the calorimeter structure. Along the direction of the beam, as shown on the left, we see a $15^{\circ}$ wedge in $\phi$, with the CHA mounted directly outside the CEM. In the transverse view to the right, the $\eta$ coverage is shown. The detector is segmented in units of $\Delta \eta=0.11$, and the resulting towers (0-9) point back to the interaction region. The Central Muon chambers are also shown. 
off light when ionized, are inserted to detect the shower of final-state particles. By interleaving layers of absorber and scintillator, the detector samples the shower and measures the total amount of energy deposited by the incident particle.

After an initial layer of aluminum at the inner radius, the CEM alternates 31 layers of $5 \mathrm{~mm}$-thick scintillator with 30 layers of $3.2 \mathrm{~mm}$-thick lead, which is clad with $0.4 \mathrm{~mm}$ aluminum on the front and back surfaces. Waveguides for the collection of light from the scintillators are mounted on the sides, just inside $5 \mathrm{~mm}$-thick steel plates which isolate the chamber. This reduces the active azimuthal coverage by $\sim 5 \%$.

The thickness of absorbing material is usually characterized in terms of the radiation length, $X_{0}$. This is the depth at which electrons, on average, have lost $\left(1-e^{-1}\right) \approx 63 \%$ of their original energy. For photons, the probability of conversion to an electron-positron pair within a distance $X_{0}$ is $7 / 9$. In general, $X_{0}$ is energydependent but for electrons and photons is roughly constant for energies above 1 $\mathrm{GeV}$. Because a particle at larger $\eta$ passes through more material, layers of acrylic are occasionally substituted for lead to maintain a constant radiation length of $18 X_{0}$ over all towers of the CEM $(|\eta|<1.1)$.

The energy resolution of the CEM towers is calculated using testbeam data and depends on the transverse energy of the electron or photon. An additional $2 \%$ error is added in quadrature to account for uncertainties due to detector geometry and the readout electronics. The overall resolution is calculated using:

$$
\frac{\sigma(E)}{E}=\sqrt{\left(\frac{13.5 \%}{\sqrt{E_{T}}}\right)^{2}+(2 \%)^{2}} .
$$

During the shower cascade process, the number of particles multiplies while the average particle energy drops until the total cross section for further particle production falls below that for internal processes (such as atomic excitation). This point is known as the shower maximum, and the depth within the material depends logarithmically on the incident energy. For the CEM, this corresponds to a depth of $\sim 6 X_{0}$ in each tower.

In order to precisely locate the shower, an orthogonal set of wires and strips, known as the Central Electron Shower-maximum (CES) detector, is placed between the 
eighth lead layer and ninth scintillator layer. These provide $r$ and $z$ information, with precision of $2 \mathrm{~mm}$ for high- $p_{T}(>40 \mathrm{GeV}$ ) electrons. This can be improved somewhat by placing additional chambers, known as the Central Pre-radiator (CPR), between the solenoid and CEM to measure shower development due to particle interaction with the magnet.

\subsubsection{Central hadronic calorimeters (CHA,WHA)}

Unlike EM calorimetry, hadronic calorimeter design rests upon the principle of nuclear interaction instead of electromagnetic forces. An incident hadron inelastically collides with nuclei inside the absorbing material of the detector and produces secondary hadrons, which interact and create more hadrons, and so on.

The distance scales over which the shower develops are much larger than for electromagnetic processes. The hadronic counterpart to radiation length in EM calorimeters is the nuclear absorption length, $\lambda_{0}$. For heavy elements, this can be a factor of 30 larger. (Lead, for instance, has $\lambda_{0}=18 \mathrm{~cm}$ versus $X_{0}=0.56 \mathrm{~cm}$.) Therefore, hadronic calorimeters are required to be much larger.

For the Central Hadronic Calorimeter (CHA), $25 \mathrm{~mm}$ layers of steel absorber are alternated with $10 \mathrm{~mm}$ layers of scintillator. Thirty-two layers of each translates to 4.7 absorption lengths across all towers $(|\eta|<0.9)$. The Wall Hadronic Calorimeter extends the pseudorapidity coverage $(0.9<|\eta|<1.3)$ and uses 15 layers of $51 \mathrm{~mm}$ thick steel and $10 \mathrm{~mm}$-thick scintillator. This depth corresponds to $4.5 \lambda_{0}$.

The resolution of the detector is also poorer than for electromagnetic calorimeters. In the EM case, most of the incident energy eventually appears as ionization from charged particles. Hadronic processes, however, can lead to production of neutral particles (neutrinos and neutrons) which escape the detector entirely or charged particles (such as muons) which do not deposit all of their energy in the calorimeter before exiting. For the energy range of $1-20 \mathrm{GeV}$, roughly $30 \%$ of the incident energy is lost to these processes.

The energy resolution is calculated for incident isolated pions. Again, a constant $3 \%$ error is added in quadrature and describes the inherent uncertainty unrelated to 
the particle momentum:

$$
\frac{\sigma(E)}{E}=\sqrt{\left(\frac{75 \%}{\sqrt{E_{T}}}\right)^{2}+(3 \%)^{2}}
$$

\subsection{Muon detectors}

The central electromagnetic and hadronic calorimeters combine for a total of 4.9 absorption lengths, which is sufficient material to filter out all but a small fraction of hadronic particles. Energetic muons with $p_{T}>1.5 \mathrm{GeV}$ are capable of penetrating the calorimetry and appear as tracks in the muon chambers.

The CDF central muon detector [24] is a collection of three subsystems - the Central Muon detector (CMU), the Central Muon Upgrade (CMP), and Central Muon Extension $(\mathrm{CMX})$ - as shown earlier in Figure 3.2. On the far ends of the detector are the Forward Muon Toroids, which were not used in this analysis. Just as with the calorimetry, we confine ourselves to the Central region.

The CMU muon chambers are placed directly behind the CEM and CHA calorimeter towers, as seen in Figure 3.7, at a radius of 3.47 meters from the beamline. The $\mathrm{CMU}$ wedge for each tower is $12.6^{\circ}$ wide, leaving $2.4^{\circ}$ gaps in $\phi$ between adjacent wedges, and each wedge is divided into three $4.2^{\circ}$ modules. The modules are $2.26 \mathrm{~m}$ long and are placed on each side of $z=0$, extending coverage out to $|\eta|<0.6$.

The modules are further subdivided into four layers of four drift cells, shown in Figure 3.8. A $50 \mu \mathrm{m}$ sense wire is placed in the center of each cell, which is filled with an argon-ethane gas mixture. The muon track ionizes the gas, and the resulting pulse height is measured at the opposite ends of the chamber. This allows determination of $z$ to within $1.2 \mathrm{~mm}$. Notice in the diagram that two cell layers are offset from the other two. Calculating $\phi$ is complicated by the ambiguity as to which side of the sense wire the muon passed. By displacing the wires by $2 \mathrm{~mm}$, the timing information of the hits is used to remove the ambiguity by looking to see which wires were hit first. Resolution is $250 \mu \mathrm{m}$ in the $r-\phi$ plane.

Two $60 \mathrm{~cm}$ steel walls are placed along the sides of the detector $(x= \pm 5.4 \mathrm{~m})$ to complement the steel return yoke of the solenoid, which covers the top and bottom 


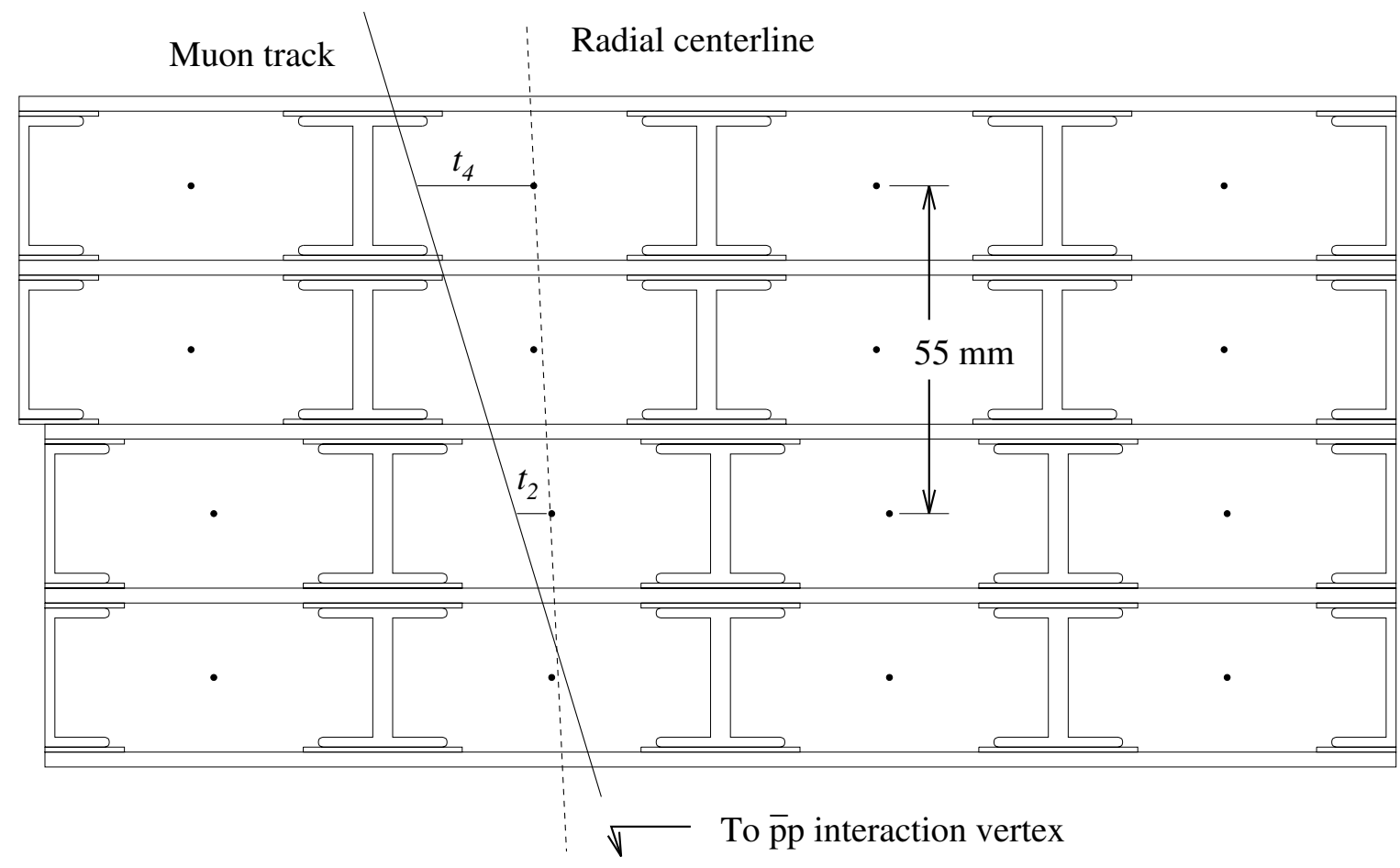

Figure 3.8: Diagram of a central muon tower. In the CMU, this subtends an angle of $4.2^{\circ}$ and consists of 16 drift cells which are $2.26 \mathrm{~m}$ long. A muon track passes through, ionizing the gas, and timing information on the hits allows reconstruction of the track. Any ambiguity in the track position is resolved by offsetting each pair of layers by $2 \mathrm{~mm}$. 
$(y= \pm 4.8 \mathrm{~m})$. These provide 3 absorption lengths of additional shielding, and muons must have $p_{T}>2.3$ to pass through. Four layers of muon chambers similar to the CMU are mounted outside the steel and constitute the Central Muon Upgrade. Because of gaps in the return yoke, there are $\phi$ gaps in the CMP centered at the top and bottom $\left(80^{\circ}<\phi<100^{\circ}\right.$ and $\left.260^{\circ}<\phi<280^{\circ}\right)$, but otherwise the CMP provides good coverage of the $\phi$ gaps in the CMU. Of the total angular coverage for $|\eta|<0.6$, approximately $84 \%$ is instrumented by the CMU, $63 \%$ by the CMP, and $53 \%$ by both.

The Central Muon Extension consists of 4 free-standing conical arches which cover $0.6<\eta<1.0$ and $71 \%$ of the $2 \pi$ angle in $\phi$. The combined angular coverage of all three muon systems is shown in Figure 3.9. Four layers of drift chambers are sandwiched between two layers of scintillator counters (known as the CSX), which acts as a trigger for the CMX system.

A track must register hits in at least 3 of the 4 layers of a detector (CMU, CMP, or CMX), and these must be aligned in $r-\phi$ and $r-z$ to within detector resolutions. The hits are then combined to form a 3-D track segment known as a muon stub. Muon tracks are then classified according to the detectors which contain stubs. CMU-only, CMP-only, and CMX-only muons are valid for our analysis. A CMU stub with a corresponding CMP stub reduces the likelihood that the track is from a pion which has punched through the hadronic calorimeter. A sample of such CMUP muons is about $95 \%$ pure.

\subsection{Event triggers}

At an experiment such as CDF, it is neither possible nor desirable to record the result of every single interaction of the $p$ and $\bar{p}$ beams. The bunch spacing of $3.5 \mu \mathrm{s}$ translates to a collision rate of $280 \mathrm{kHz}$. A typical event description is on the order of $100 \mathrm{kBytes}$, so the system would be required to archive data at a rate of $30 \mathrm{MB} / \mathrm{s}$. The network and storage capacity would become overwhelmed, assuming that the entire detector could actually be read out that quickly.

Compared to the $50 \mathrm{mb}$ total inelastic cross section, most processes of interest have cross sections five to ten orders of magnitude smaller and comprise a tiny percentage 


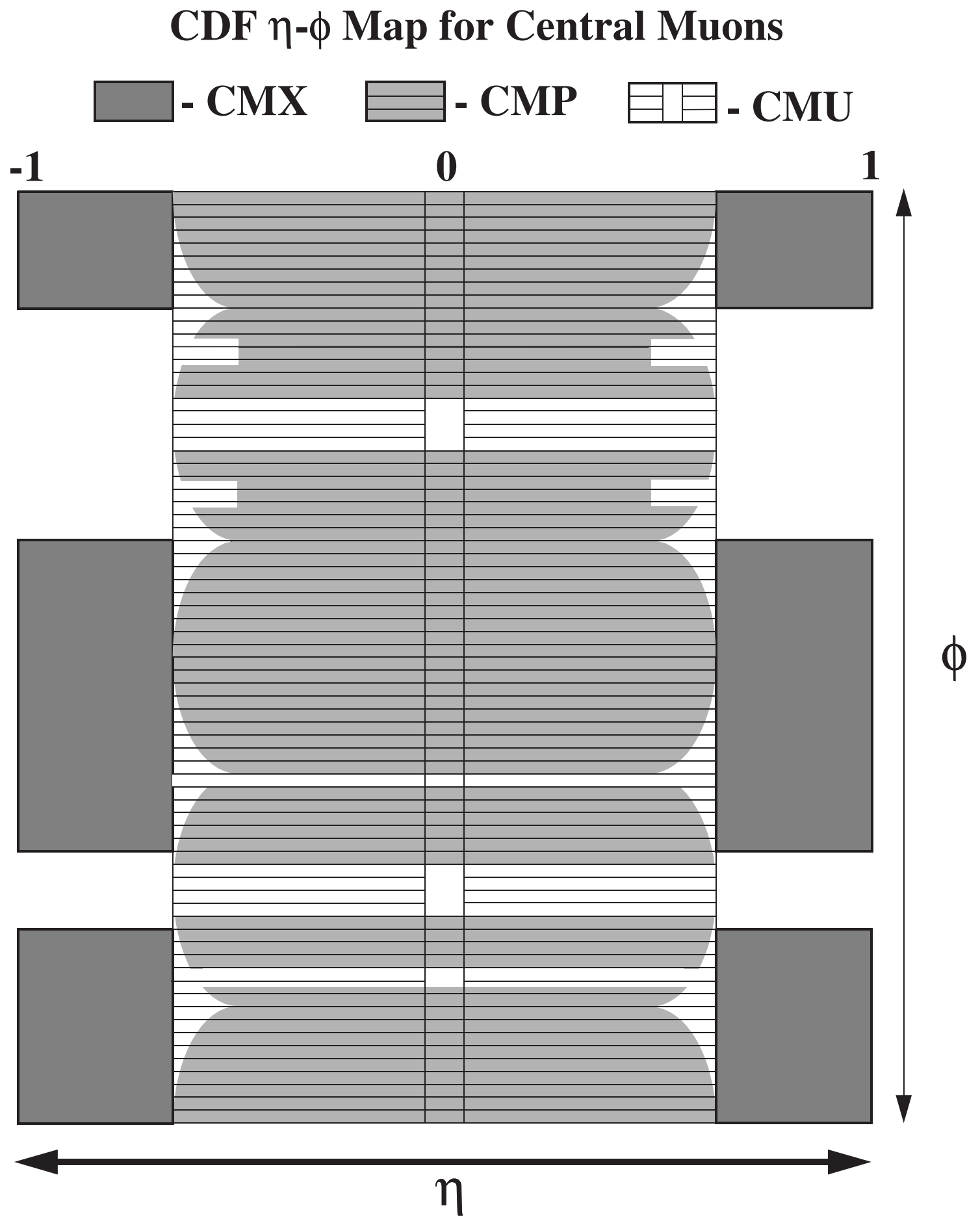

Figure 3.9: $\eta-\phi$ coverage of the CDF central muon detector systems. The $\phi$ gaps in the CMP correspond to gaps in the return yoke, while the large $\phi$ gaps in the CMX occur where the CMX intersects with the floor (large gap) and the Tevatron (small gap). 


\begin{tabular}{|ccr|}
\hline \hline Trigger level & Output rate & Deadtime \\
\hline (Beam crossings) & $280 \mathrm{kHz}$ & - \\
Level 1 & $1-2 \mathrm{kHz}$ & $0 \%$ \\
Level 2 & $20-35 \mathrm{~Hz}$ & $4 \%$ \\
Level 3 & $3-8 \mathrm{~Hz}$ & $10 \%$ \\
\hline \hline
\end{tabular}

Table 3.2: Summary of the event rates and deadtimes for the CDF trigger stages.

of all the interactions produced. Therefore, a trigger system was devised to throttle the data rate down to a manageable size and to separate the more interesting events into well-defined datasets. At CDF, there are three trigger levels, with multiple trigger paths to accommodate a wide variety of proposed physics analyses. Specifications for the trigger are provided in Table 3.2.

The first set of triggers, Level 1, is hardware based and uses only information from the calorimeter (for inclusive electrons and jets) and muon detectors (for inclusive muons). Analog signals are sent directly from the detector preamplifiers into logic circuits, which allows for fast decision-making. No tracking system information is available at Level 1 . The first trigger requires that the energy in a single calorimeter tower be above a prescribed threshold, which is dependent on the particular calorimeter system. In the central region, this was 6 (8) GeV in Run Ia (Ib) for the CEM and 8 (12) GeV for the CHA. The other thresholds are listed in Table 3.3.

Without tracking information, the muon trigger requires a stub in the CMU or CMX and examines the timing information of the sense wire hits to determine the muon momentum. For the CMU, the stub $p_{T}$ must be $>6 \mathrm{GeV}$ and must either match a corresponding stub in the CMP or, if there is a gap in the CMP coverage, be "in-time" with the beam crossing according to the hadronic calorimeter TDCs. In the CMX, the track stub must satisfy $p_{T}>10 \mathrm{GeV}$, have a positive trigger in the CSX, and also be "in-time". A minimum energy of $300 \mathrm{MeV}$ in the CHA tower cor- 


\begin{tabular}{|cll|}
\hline \hline Calorimeter & Run Ia & Run Ib \\
\hline CEM & $6 \mathrm{GeV}$ & $8 \mathrm{GeV}$ \\
CHA & 8 & 12 \\
PEM & 8 & 11 \\
PHA & 25 & 51 \\
FEM & 8 & 51 \\
FHA & 25 & 51 \\
\hline \hline
\end{tabular}

Table 3.3: Single tower energy thresholds for the Level 1 calorimeter triggers in Run Ia and $\mathrm{Ib}$ for all electromagnetic and hadronic calorimeter systems.

responding to the CMU or CMX track position reduces the possibility of coincidental hits in the muon chambers.

This decision requires less than $3.5 \mu s$, so the trigger does not incur any deadtime, during which the detector would be be forced to ignore subsequent beam crossings. After Level 1, the initial event rate of $280 \mathrm{kHz}$ is reduced down to 1 or $2 \mathrm{kHz}$ for luminosities of $1-2 \times 10^{31} \mathrm{~cm}^{-2} \mathrm{~s}^{-1}$. After a successful Level 1 trigger, data from all detector systems is passed to Level 2.

Level 2 is also hardware based and requires anywhere from $20-30 \mu s$ for a typical event to $\sim 100 \mu s$ for a more complex event containing large amounts of calorimeter and tracking activity. In the absence of a data pipeline, further event readout is suspended, and the deadtime is around 4\%. At this level, the trigger performs clustering of calorimeter energy and uses tracking information from the CTC to form electron and muon candidates.

The hardware cluster finder identifies primary towers with $E_{T}>3 \mathrm{GeV}$ and then scans the adjoining towers in $\eta$ and $\phi$, adding those with $E_{T}>1 \mathrm{GeV}$ to the cluster. A list of all clusters is produced, including data on total $E_{T}$, average $\eta$ and $\phi$, and width 
in $\eta$ and $\phi$. Events containing jet clusters with $E_{T}>20,50,70,100 \mathrm{GeV}$ are accepted and form the basis of the CDF JET20, JET50, JET70, and JET100 samples.

Track reconstruction is performed in hardware by the Central Fast Tracker (CFT) [25]. High $p_{T}$ tracks are located using the $r-\phi$ layers of the CTC, and the momentum resolution is $\delta p_{T} / p_{T}=3.5 \%\left(\frac{p_{T}}{\mathrm{GeV}}\right)$. Level 2 muons are identified by a CFT track which matches to within $5^{\circ}$ of a muon stub found in the Level 1 trigger. Electrons are characterized by CFT tracks with $p_{T}>9.2 \mathrm{GeV}$ and a CEM cluster of $E_{T}>9$ $\mathrm{GeV}$. The CEM cluster differs from a jet cluster in that a central tower of $9 \mathrm{GeV}$ is required and neighboring towers must have at least $7 \mathrm{GeV}$. The hadronic energy in an electron cluster should be a fraction of the EM energy deposited, so the ratio of CHA/WHA to CEM energy must be $<0.125$.

The Level 3 trigger accepts events from Level 2 and performs the final evaluation before an event is recorded. A "farm" of six Silicon Graphics workstations, each with eight RS4000 CPUs, runs the full CDF reconstruction software which is also used for offline analysis. Here, multiple trigger paths are supplied to provide data streams for the different event signatures of interest to the various physics groups. The output rate is constrained only by the physical limitation of the $8 \mathrm{~mm}$ magnetic tape drives used as storage media: $3-5 \mathrm{~Hz}$ in Run Ia and $\sim 8 \mathrm{~Hz}$ in Run Ib.

In Level 3, CTC tracks for the electron and muon candidates are fully reconstructed in 3-D and are required to match an electromagnetic cluster or muon stub. Events which pass this trigger form the basis of the inclusive electron and inclusive muon datasets which were used in this analysis. These will be described in more detail in Section 5.1. 


\section{Chapter 4}

\section{Event Selection}

As described in Section 2.8, we expect to observe gluino pair production at the Tevatron using top quarks produced by gluino decay to top and scalar top $(\tilde{g} \rightarrow$ $t \overline{\tilde{t}}$. The Majorana nature of the gluino presents the unique opportunity to discover supersymmetry in like-sign top events. Of the various top event signatures, the one which offers the greatest ability to discern $\tilde{g} \tilde{g}$ production from Standard Model $t \bar{t}$ is the top dilepton channel. Borrowing from our understanding of SM top dilepton events, we search for events containing two high momentum leptons, in this case with identical charge; two energetic jets from the $b$ quarks produced by top; and missing energy from the neutrinos created through the leptonic decay of $W(W \rightarrow l \nu)$.

Many Run I analyses have been performed by the CDF collaboration, and over time a general consensus has formed as to how different physics signals manifest themselves in the detector. Extensive testbeam studies of the individual detector components have provided an understanding of how various physics objects (electrons, muons, photons, jets) appear when they pass through the detector. Intensive efforts have been undertaken to determine the optimum selection criteria used to distinguish these objects from one another, as well as from background activity and electronic noise. In reality, the detector systems and event reconstruction software suffer from inefficiencies, and the selection methods are not guaranteed to be perfect. Yet, they have been designed such that a small change in the selection criteria does not adversely affect the results of any analysis. If the end result critically depended upon fine tuning 
of the selection criteria, any conclusions drawn from the analysis would be highly suspect.

In this chapter, we describe the methods used to identify events of interest for our gluino search. Many of the selection criteria below have been successfully applied in searches for, and systematic studies of, the top quark and are presented here without further justification. Where appropriate, we refer the reader to the original studies which determined or optimized a particular cut. In the chapters which follow, we shall present the results obtained by applying the event selection procedures below to Run I data and to Monte Carlo simulations of $\tilde{g} \tilde{g}$ production.

\subsection{Electron identification}

Electrons appear as tracks in the SVX and CTC and then deposit most, if not all, of their energy in the electromagnetic calorimeter. A high percentage of the energy cluster is concentrated in a single tower, known as the seed tower, and any remainder appears in adjacent towers. For electron ID, the primary tower must contain at least $9 \mathrm{GeV}$ of energy, and adjacent towers in $\eta$ and $\phi$ are required to have more than 7 $\mathrm{GeV}$ to be included in the cluster. Test beam studies have been performed to map the detector performance, and the strip-by-strip shower profile in the CES obtained from test electrons is used as a comparison template for identifying real electrons in the data.

Electron identification is more complicated at high $\eta$ due to the lack of tracking coverage. For an energy cluster in the plug or forward region, the absence of a matching CTC track makes it difficult to distinguish electrons from photons and jets, all of which deposit energy in the EM calorimetry. Therefore, this analysis only accepts electrons in the central region $(|\eta|<1.1)$.

Identification of electrons involves searching for a high- $p_{T}$ track in the CTC, a cluster of energy in the CEM calorimeter that is aligned with the track, and a corresponding signal in the shower-max strip detector (CES). The purity of the sample is improved by first imposing geometrical requirements on the acceptance to avoid detector edges and gaps. Then quality cuts are applied to the physical quantities 
measured by the tracking chambers and calorimeters to separate electrons from other physics objects.

\subsubsection{Fiducial requirements}

An electron that showers near the CEM detector boundaries or gaps will be mismeasured if a significant amount of energy is deposited into inactive regions of the detector. To improve the overall quality of electrons selected, the following geometrical requirements (often referred to as fiducial cuts) are placed on electromagnetic clusters:

- $\phi$ boundaries of CEM wedges: The shower position as measured by the shower max detector must not line up near the $\phi$ gap of the calorimeter wedge. At the radius of the $\mathrm{CES}$, the width of the $\mathrm{CEM}$ wedge is $48.5 \mathrm{~cm}$. The shower position is not allowed to be within $3.2 \mathrm{~cm}$ of the edges, which reduces the geometrical coverage by $13 \%$.

- $\eta$ boundaries of CEM wedges: Electrons are allowed to cross boundaries in $\eta$.

- $z=0\left(90^{\circ}\right)$ gap : At the center of the detector, there exists a gap between the east and west cylindrical halves of the calorimeter. The cluster position in $z$ must be at least $9 \mathrm{~cm}$ from this gap. This requirement removes about $5 \%$ of central electrons.

- Large $\eta$ boundary: The seed tower of the EM cluster cannot be tower 9, which lies on the outside edge of the central calorimeter (shown in Figure 3.7) and provides the highest $\eta$ coverage. An EM cluster centered in tower 9 would likely be mismeasured due to energy loss outside the detector.

- The Chimney module: In the $\phi$ wedge between $75^{\circ}$ and $90^{\circ}$, the calorimeter contains only 7 full towers (0-6) and a partial tower (tower 7). Towers 8 and 9 are not instrumented, and the gap in coverage corresponds to $0.77<\eta<1.0$. This region of the detector contains connections to the cryogenic cooling system 
and allows access to the solenoid. The seed tower of a cluster in this $\phi$ range cannot be tower 7 .

\subsubsection{Tracking and calorimeter ID cuts}

Once an electromagnetic cluster passing the fiducial cuts has been identified, the reconstruction software searches for at least one 3-D track in the CTC which points to the cluster. The cluster and matching track(s) are then subjected to a series of quality cuts to identify those most likely originating from an electron. The kinematic variables used are described in detail below:

- $E_{T}$, transverse energy: This is the total electromagnetic energy measured among all towers included in the CEM cluster. The value used is the raw energy measurement and is not corrected for variations in response across towers.

- $p_{T}$, transverse momentum: The momentum of the CTC track, as measured in the transverse plane $(r-\phi)$, is calculated from the curvature of the particle trajectory due to the magnetic field of the solenoid.

- $E / p$, ratio of the EM calorimeter energy to the track momentum: As the electron is accelerated by the magnetic field, energy is radiated away through bremsstrahlung. Resulting measurements of the momentum and energy are reduced, though some energy is recovered if the electron and bremsstrahlung products are clustered together in the calorimeter. This leads to an $E / p$ ratio greater than 1.

- $E_{H A D} / E_{E M}$, ratio of hadronic to electromagnetic calorimeter energy: An electron is unlikely to fully penetrate the material of the CEM and typically deposits little to no energy in the hadronic calorimeter (CHA or WHA). This ratio should be on the order of $5 \%$.

- $L_{s h r}$, lateral shower profile: The amount of energy in adjacent CEM towers compared to that in the seed tower must be similar to the distribution measured 
for test beam electrons, to within detector resolution and uncertainties in the test beam data. The comparison is calculated using

$$
L_{s h r}=0.14 \sum_{i} \frac{E_{i}^{o b s}-E_{i}^{e x p}}{\sqrt{(0.14 \sqrt{E})^{2}+\sigma_{E_{i}^{e x p}}^{2}}}
$$

where the sum is performed over all adjacent towers $i$ in the cluster. $E_{i}^{o b s}$ is the observed energy in tower $i, E_{i}^{e x p}$ is the expected value from test beam data, $0.14 \sqrt{E}$ is the energy resolution of the CEM, and $\sigma_{E_{i}^{e x p}}^{2}$ is the uncertainty in the expected energy. The terms in the summation are allowed to be positive or negative. An overall excess in the observed energy distribution indicates that the original particle may be something other than an electron, so an upper bound is placed on $L_{s h r}$.

- $|\Delta x|$ : The CTC track trajectory is extrapolated out to the CES chamber, and the position is compared to the shower-max location measured by the CES wires parallel to the beam. $|\Delta x|$ is the distance between the track and shower-max centroid in the $r-\phi$ plane.

- $|\Delta z|$ : This is the same as for $|\Delta x|$, except here the distance is between the extrapolated position in $z$ of the CTC track and the shower position measured by CES strips perpendicular to the beam.

- z-vertex match: The CTC track is extrapolated back to the beamline, and the $z$ position that is obtained is compared to the event vertex (or vertices) identified by the VTX. (In an event containing multiple interactions, where more than one event vertex is identified, the closest vertex is used.) An event vertex must lie within $5 \mathrm{~cm}$ to be associated with the track and is required to be within $|z| \leq 60 \mathrm{~cm}$ of the center of the interaction region.

- $\chi_{\text {strip }}^{2}$ : The shower profile along $z$ in the 11 CES strips perpendicular to the beamline must compare favorably with the profile observed for test beam electrons. 
In early analyses involving the top quark, only one set of cuts existed for electron ID. Subsequent studies found that, in order to increase the overall event acceptance, the cuts could be relaxed slightly for any secondary electrons in the event, without significant loss in quality. This created two classifications of electrons:

- Tight Central Electron (TCE) and

- Loose Central Electron (LCE).

Similarly, tight and loose categories will be designated later for muons, and the selection criteria for dilepton events will require at least one lepton to belong to a tight category.

In dielectron events, the primary electron must pass the tighter set of Lepton ID requirements to maintain the purity of the sample. Any electron which fails the tight set of cuts is re-examined to determine whether it passes the looser cuts. In this manner, the TCE and LCE categories are mutually exclusive. Both sets of cuts are summarized in Table 4.1.

For the loose electron category, the maximum values allowed for $E / p$ and $E_{H A D} / E_{E M}$ are increased, and the $\chi_{\text {strip }}^{2}$ requirement is removed. The relaxed $E_{H A D} / E_{E M}$ cut features a sliding scale maximum with the addition of a term proportional to the electron energy. This allows for the fact that, as the incident energy of an electron increases, it is more likely to punch through to the hadronic calorimeter and deposit energy there.

Distributions of the variables used for electron ID are shown in Figure 4.1. These plots were created from dielectron events in Run I data which are candidates for $Z^{0} \rightarrow$ $e^{+} e^{-}$. In these events, a primary electron which passes the tight set of electron ID cuts is paired with other electrons which must pass only a minimal set of requirements, not the full set of Lepton ID cuts. For those events where the invariant mass of the pair is within $15 \mathrm{GeV}$ of the $Z^{0}$ mass $\left(75 \leq m_{l l} \leq 105 \mathrm{GeV}\right)$, the kinematic variables of the second electron are plotted. The lepton ID cuts for each variable are indicated on the plots. 


\begin{tabular}{|c|c|c|}
\hline Variable & Cut & Comments \\
\hline$E_{T}$ & $>20 \mathrm{GeV}$ & \\
\hline$p_{T}$ & $>10 \mathrm{GeV} / \mathrm{c}$ & \\
\hline$E / P$ & $\begin{array}{l}<1.8 \\
<4.0\end{array}$ & $\begin{array}{l}\text { for TCE } \\
\text { for LCE }\end{array}$ \\
\hline$E_{H A D} / E_{E M}$ & $\begin{array}{c}<0.05 \\
<0.05+\frac{0.045 \mathrm{xE}}{100}\end{array}$ & $\begin{array}{l}\text { for TCE } \\
\text { for LCE }\end{array}$ \\
\hline$L_{s h r}$ & $<0.2$ & \\
\hline$|\Delta x|$ & $<1.5 \mathrm{~cm}$ & \\
\hline$|\Delta z|$ & $<3.0 \mathrm{~cm}$ & \\
\hline$z$-vertex match & $<5.0 \mathrm{~cm}$ & \\
\hline$\chi_{\text {strip }}^{2}$ & $<10.0$ & for TCE only \\
\hline
\end{tabular}

Table 4.1: Electron identification requirements, with distinctions between tight (TCE) and loose (LCE) category electrons specified. 

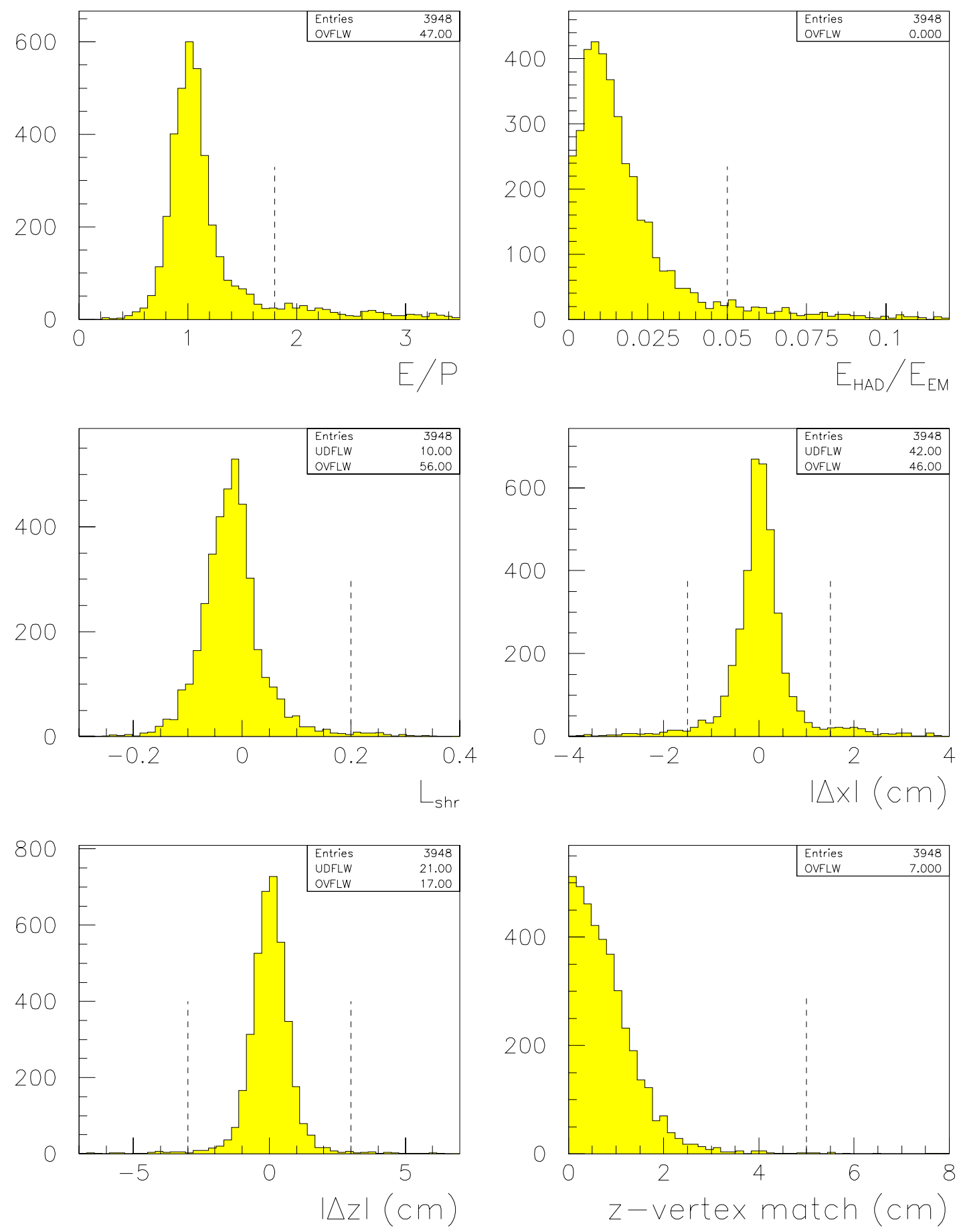

Figure 4.1: Distributions of central electron (TCE and LCE) identification variables, where the dashed line shows where the cut is placed. 


\subsubsection{Conversion removal}

The largest background for high- $E_{T}$ electrons comes from photon conversions. In a Run Ia inclusive electron sample with $E_{T}>18 \mathrm{GeV}$, they account for roughly $30-40 \%$ of events.

Photons are produced in the initial collision, via bremsstrahlung, or as a product of particle decays. As they traverse the detector volume, they interact with material in the beampipe, detector components, structure supports, and solenoid, where they convert into electron-positron pairs $\left(\gamma \longrightarrow e^{+} e^{-}\right)$. In the outer regions of the detector, photons deposit energy in the EM calorimeters but are easily identified by the absence of the matching track. In the tracking region, a photon conversion appears as a pair of closely spaced tracks.

A photon removal routine [26, 27] is employed at the lepton ID level. This algorithm identifies an electron as a conversion by searching for a second opposite-sign track such that the two have a small separation in the azimuthal plane $(\mid \delta(r-\phi)<$ $0.3 \mathrm{~cm} \mid)$ and in polar angle $(|\delta \cot \theta<0.06|)$. The location of the most likely conversion point is determined, and a cut is applied to the calculated radius: $-20<R_{\text {conv }}<$ $50 \mathrm{~cm}$. (Negative values for the conversion radius may occur for closely spaced tracks or arise out of tracking inefficiencies.) Distributions of the photon conversion variables are shown in Figure 4.2.

The above prescription assumes that both tracks from a photon conversion are identified. This is not always true and leads to some inefficiency in the removal routine. To account for this possibility, an alternate requirement is placed on electron candidates using information from the VTX. The VTX occupancy is defined as the ratio of observed VTX hits to the number expected from the CTC track trajectory. For electrons where the expected number of hits is three or greater, the occupancy must be $\geq 0.2$. If only one or two hits are anticipated and none are registered, the electron is not discarded. Electrons which fail either or both cuts are flagged as conversions.

The algorithm is $91 \%$ efficient [26] at removing conversion electrons from consideration. This comes at the price of a limited number of electrons from other sources 


\section{Run 1a Inclusive Electrons, $\mathrm{E}_{\mathrm{T}}>18$}
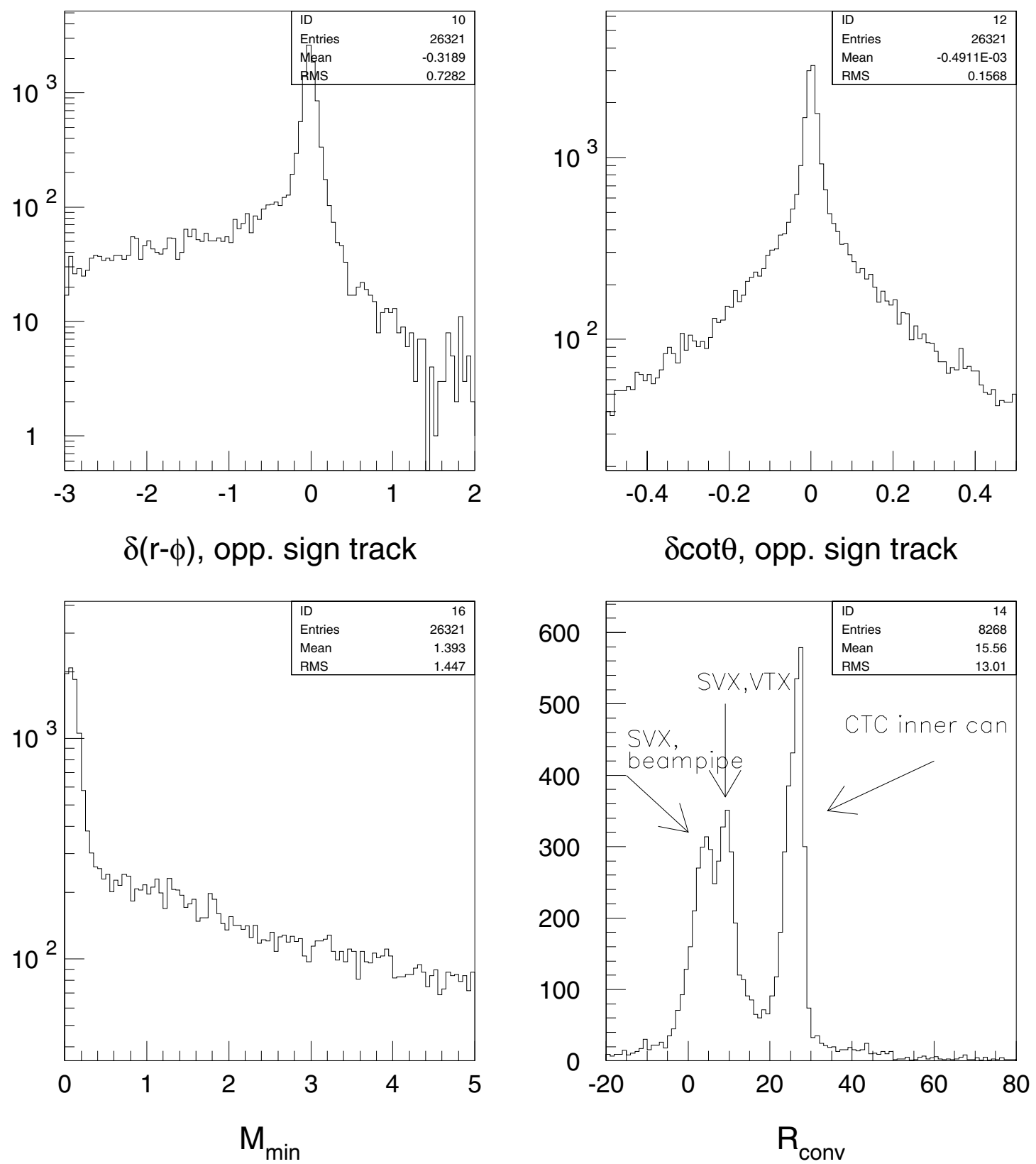

Figure 4.2: Conversion electrons are identified by searching for two electron tracks which are closely spaced in $r-\phi(|\delta(r-\phi)<0.3 \mathrm{~cm}|)$ and $\operatorname{in} \theta(|\delta \cot \theta<0.06|)$. Also shown is the two-track invariant mass. After application of the angular cuts, the conversion radius distribution nicely illustrates the location of the beampipe, the SVX and VTX cabling and supports, and the CTC inner radius. Taken from Ref. [26]. 
which are misidentified as conversions, especially due to inefficiencies in the VTX that lower the occupancy. The overefficiency, the percentage of non-conversion electrons which are removed by the above algorithm, has been calculated using electrons from $W^{ \pm}$and $Z^{0}$ events. For these samples, the overefficiency was found to be $2.3 \pm 0.6 \%$ and $2.2 \pm 0.6 \%$, respectively. This has implications for the acceptance rate in the top dilepton channel and will be discussed further in Section 7.6.1.

\subsection{Muon identification}

Muons are minimum ionizing particles and are characterized by little or no energy deposition in the electromagnetic and hadronic calorimeters. A muon is identified by a high- $p_{T}$ track reconstructed from hits in the tracking region, and possibly includes hits in one or more of the muon chambers. Occasionally the muon is directed toward a gap in the detector coverage or fails to register sufficient hits to create a muon stub, in which case the tracking information becomes crucial. Just as with electrons, the necessity of a well-defined track limits the range of muon identification to the central portion of the detector.

Recall that CDF contains three central muon systems: the Central Muon detector (CMU), Central Muon Upgrade (CMP), and Central Muon Extension (CMX). Tracks with a corresponding stub in one or more of these detectors are considered the highest quality muon candidates. However, a high- $p_{T}$ track which has no stub but deposits little energy in the calorimeter is also a viable candidate, as long as the track passes a few additional requirements. This type of muon is known as a central minimum ionizing track, or CMI.

Muons in this analysis have three separate classifications:

- Tight Central Muon (TCM)

- Central Muon Extension (CMX) and

- Central Minimum Ionizing (CMI). 
The TCM category includes tracks with a stub in the CMU only, in the CMP only, or in both the CMU and CMP (denoted by CMUP). While CMX muons are treated separately, both the TCM and CMX classes are considered to be tight, the same as TCE electrons. CMI muons, because they lack a muon stub, are classified as loose and are on par with LCE electrons.

\subsubsection{Fiducial requirements}

The geometrical requirements for muons are simpler than those for electrons. Electrons are primarily defined by a large energy deposit in the EM calorimeter and very little in the hadronic calorimeter. Gaps in the CEM and edges of calorimeter wedges are avoided to ensure that the shower development is well within the active region of the detector. Meanwhile, gaps in the CHA or WHA should be avoided because they mask the presence of hadronic energy, which causes physics objects such as jets to appear more "electron-like."

In contrast, muons are characterized by a track and lack of significant calorimeter energy. Muons in the TCM and CMX classes are automatically fiducial because they have a valid matching stub in one of the muon detectors. Thus, the geometrical requirements in the $\mathrm{CEM}$ and $\mathrm{CHA} / \mathrm{WHA}$ lose their importance. In the absence of a muon stub, the geometry becomes more of an issue. An electron or jet can masquerade as a muon by passing through a gap in the calorimetry or along a segmentation boundary between towers. The resulting underestimate of the calorimeter energy creates an object that appears to be "muon-like." Therefore, a CMI will only be considered for the analysis if the track trajectory is well within the active volume of the calorimeter.

The shower depth of a muon differs greatly from that of an electron, so the CES shower-max detector is not useful in determining the location for a muon. Instead, the CTC track trajectory is extrapolated into the calorimeter to the CES location, and this position is used for the geometrical cuts. The following fiducial requirements are placed on CMIs:

- $\phi$ boundaries of CEM wedges: The extrapolated track position at the CES must 
not be located near the $\phi$ gaps between calorimeter wedges. The width of the tower at the CES is $48.5 \mathrm{~cm}$, and the track position must be $2.5 \mathrm{~cm}$ away from the edge.

- $z=0\left(90^{\circ}\right)$ gap: The track position must lie at least $9 \mathrm{~cm}$ from the central $(z=0)$ gap between the two halves of the calorimeter.

- The Chimney module: The CTC track cannot pass through the Chimney module defined earlier for electrons.

- $|\eta|<1.2$ : The pseudorapidity of the detector position must be within the central region.

- Corner region: The solenoid shares a boundary with the plug EM calorimeter in the eta range $1.06<|\eta|<1.12$. This is known as the corner region. Any CTC tracks passing through this portion of the detector are discounted because the EM energy cannot be accurately measured, making it difficult to distinguish a muon from other particles.

The active regions of the muon systems are best visualized by looking at CTC tracks for muons in the three categories (TCM, CMX, and CMI). Figure 4.3 beautifully demonstrates the pseudorapidity and azimuthal coverage of the CMU, CMP, and CMX detectors. The segmentation in $\phi$ of the CMU and CMP is readily apparent, as are the large $\phi$ gaps in the CMX. Peaks in the distribution of minimum ionizing tracks coincide with detector boundary regions and gaps in the muon coverage. The effect of the corner region cut appears as a gap in the CMI distribution at high $\eta$.

In Figure 4.4, we focus on the TCM category and show the $\eta$ and $\phi$ distributions versus the type of muon stub identified (CMU and CMP, CMU only, or CMP only). These are dominated by muons with matching stubs in both the CMU and CMP. The large concentrations of CMU only tracks in $\phi$ correspond to gaps in the CMP, and the smaller clusters of CMP only muons are aligned with the regular $\phi$ gaps of the CMU. 

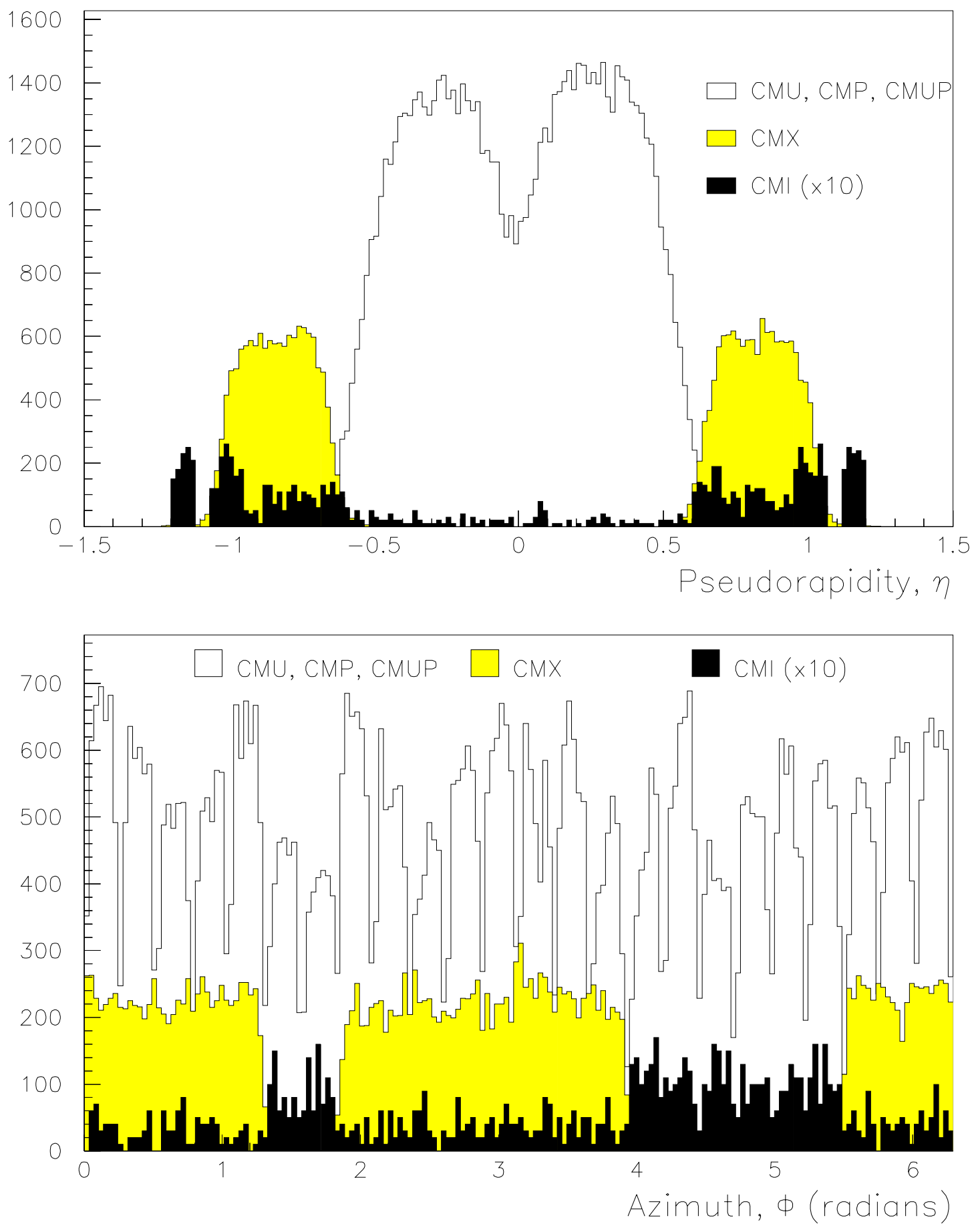

Figure 4.3: Distributions of CTC track pseudorapidity $(\eta)$ and azimuthal angle $(\phi)$ for all inclusive muons (TCM, CMX, and CMI) identified by the Lepton ID requirements. The CMI distribution is enlarged by a factor of 10 to enhance its visibility. 

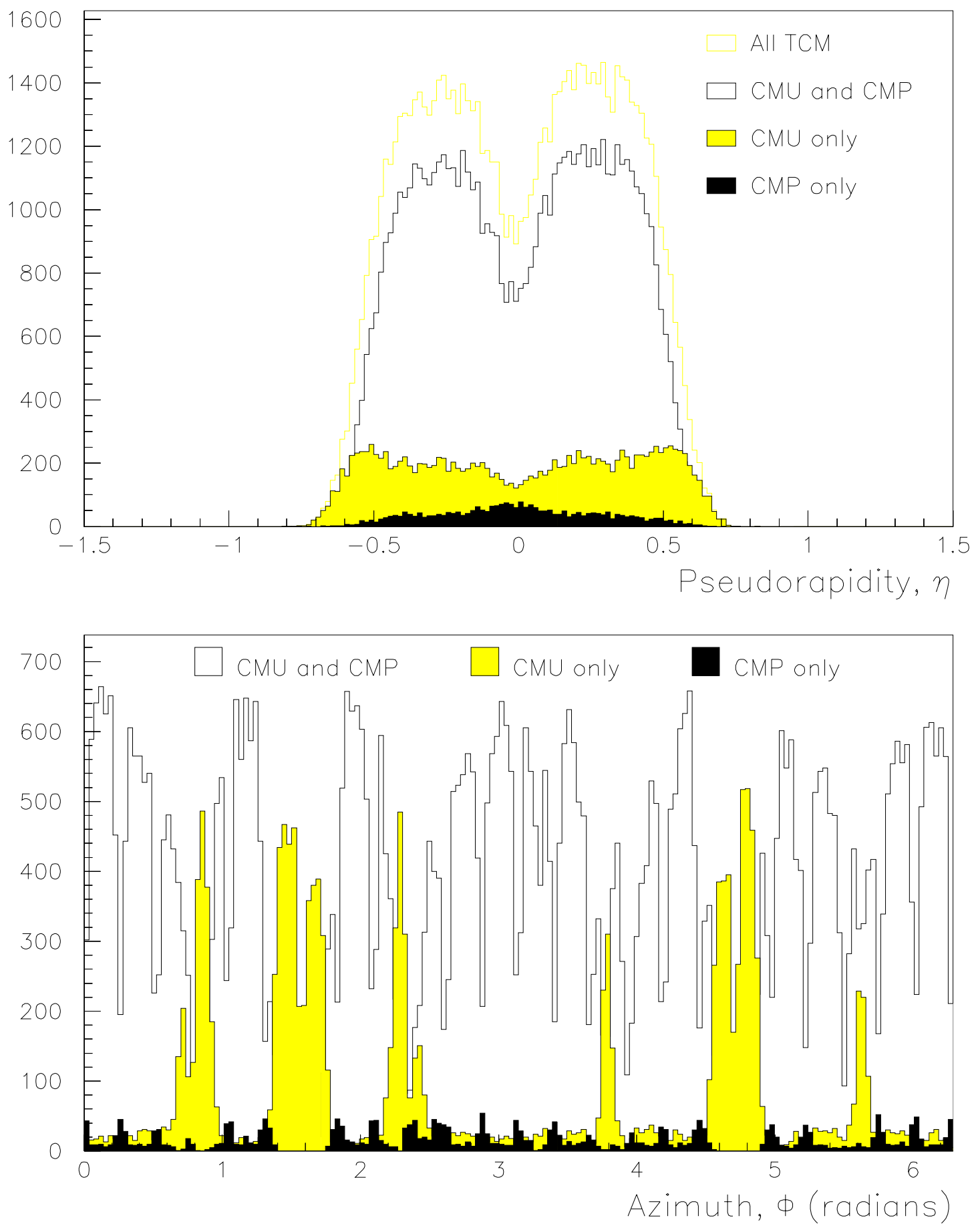

Figure 4.4: Distributions of CTC track $\eta$ and $\phi$ for all TCM muons, separated according to the type of muon stub associated with the track: matching CMU and CMP stubs, CMU only, and CMP only. Gaps in the CMP coverage appear as clusters of CMU only muons, and vice versa. 


\subsubsection{Track, calorimeter, and muon stub ID cuts}

The requirements placed on muon candidates are similar for the TCM and CMX categories. These focus on the quality of the matching track and the lack of significant calorimeter energy. The only difference between the two types lies in the matching of track and muon stub, where the cut values are chamber-dependent. The selection cuts for TCM and CMX muons are summarized in Table 4.2, and the kinematic variables are described below:

- $p_{T}$, transverse momentum of the CTC track: For electrons, the momentum is calculated solely from position measurements in the wire chamber. For muons, the momentum is beam constrained, which means that the track parameters are recalculated with the requirement that the track also pass through the beam position in $x$ and $y$.

- $E_{E M}$, electromagnetic calorimeter energy: Muons are minimum ionizing and should deposit very little energy in the CEM.

- $E_{H A D}$, hadronic calorimeter energy: The larger number of radiation lengths presented by the absorbing material of the CHA and WHA increases the likelihood that a particle will lose energy through ionization. The amount of energy deposition, while greater than in the CEM, is expected to be small for muons.

- $d_{0}$, impact parameter: The impact parameter is the distance of closest approach between the refit track and the beam axis in the $r-\phi$ plane.

- z-vertex match: Just as for electrons, the muon track is extrapolated back to the beamline, and the resulting vertex should coincide with an event vertex identified by the VTX. The associated event vertex must lie within $60 \mathrm{~cm}$ of the center of the interaction region.

- $|\Delta x|_{C M U},|\Delta x|_{C M P},|\Delta x|_{C M X}$ : For a track to be selected as a TCM or CMX muon, at least one matching muon stub must be located in the CMU, CMP, or CMX. The position of the extrapolated track is compared to any available stubs, and $|\Delta x|_{i}$ is the distance between the two as measured in the $r-\phi$ plane. 


\begin{tabular}{|ccc|}
\hline \hline Variable & Cut & Comments \\
\hline$p_{T}$ & $>20 \mathrm{GeV} / \mathrm{c}$ & beam contrained \\
$E_{E M}$ & $<2 \mathrm{GeV}$ & \\
$E_{H A D}$ & $<6 \mathrm{GeV}$ & \\
$E_{E M}+E_{H A D}$ & $>0.1 \mathrm{GeV}$ & \\
Impact parameter $\left(d_{0}\right)$ & $<3 \mathrm{~mm}$ & \\
$z-$ vertex match & $<5 \mathrm{~cm}$ & \\
$|\Delta x|_{C M U}$ & $<2 \mathrm{~cm}$ & for TCM only \\
$|\Delta x|_{C M P}$ & $<5 \mathrm{~cm}$ & for TCM only \\
$|\Delta x|_{C M X}$ & $<5 \mathrm{~cm}$ & for only \\
\hline \hline
\end{tabular}

Table 4.2: TCM and CMX muon identification requirements 
If a muon stub cannot be located, the track-stub matching requirement is replaced by more restrictive cuts on the track and the calorimeter energy. The first is designed to verify that the momentum and energy measurement are comparatively clean and unaffected by underlying activity in the event. The second calls for a larger number of hits in the CTC to ensure accurate reconstruction of the track. The selection criteria for minimum ionizing tracks are summarized in Table 4.3, and the new requirements are described in detail below:

- $I_{t r k}$ and $I_{c a l}$, track and calorimeter isolation: A muon will leave a well-defined track in the CTC and little energy deposition in the calorimeter. Because there is no confirming stub in the muon chambers, the track and calorimeter are required to be "isolated" from any other tracks or energy clusters. The way in which this is quantified is described in more detail in Section 4.6. In short, a cone of fixed size is centered on the muon track. The transverse momentum is summed for all tracks inside the cone other than the original, as is the energy of any additional energy clusters in the calorimeter. The track (calorimeter) isolation is defined as the ratio of the extra momentum (energy) to the original muon momentum (energy). Both the track and calorimeter isolation are required to be small $(<10 \%)$.

- Number of axial and stereo superlayers (SL): To increase our confidence in the quality of the CTC track, the total number of superlayers containing hits is required to be above a higher threshold. To ensure accurate tracking in all three dimensions, minima are also set for the number of axial superlayers and the number of stereo superlayers.

Distributions of the physics quantities used to identify CMU, CMX, and CMI candidates are shown in Figure 4.5, Figure 4.6, and Figure 4.7, respectively. In this case, we have chosen Run I events which are candidates for $Z^{0} \rightarrow \mu^{+} \mu^{-}$to create the plots. The $Z^{0}$ identification algorithm selects events with a primary muon that passes the selection criteria for TCM or CMX muons. If a secondary muon satisfying a less stringent set of cuts is found and the invariant mass lies within the $Z^{0}$ window 


\begin{tabular}{|c|c|c|}
\hline Variable & Cut & Comments \\
\hline$p_{T}$ & $>20 \mathrm{GeV} / \mathrm{c}$ & beam constrained \\
\hline$E_{E M}$ & $<2 \mathrm{GeV}$ & \\
\hline$E_{H A D}$ & $<6 \mathrm{GeV}$ & \\
\hline$E_{E M}+E_{H A D}$ & $>0.1 \mathrm{GeV}$ & \\
\hline Impact parameter $\left(d_{0}\right)$ & $<3 \mathrm{~mm}$ & \\
\hline$z$-vertex match & $<5 \mathrm{~cm}$ & \\
\hline$I_{t r k}$ and $I_{c a l}$ & $<0.1$ & \\
\hline Number of axial SL & $\geq 3$ & \\
\hline Number of stereo SL & $\geq 2$ & \\
\hline Total number of SL & $\geq 6$ & \\
\hline
\end{tabular}

Table 4.3: Minimum ionizing (CMI) particle identification requirements 

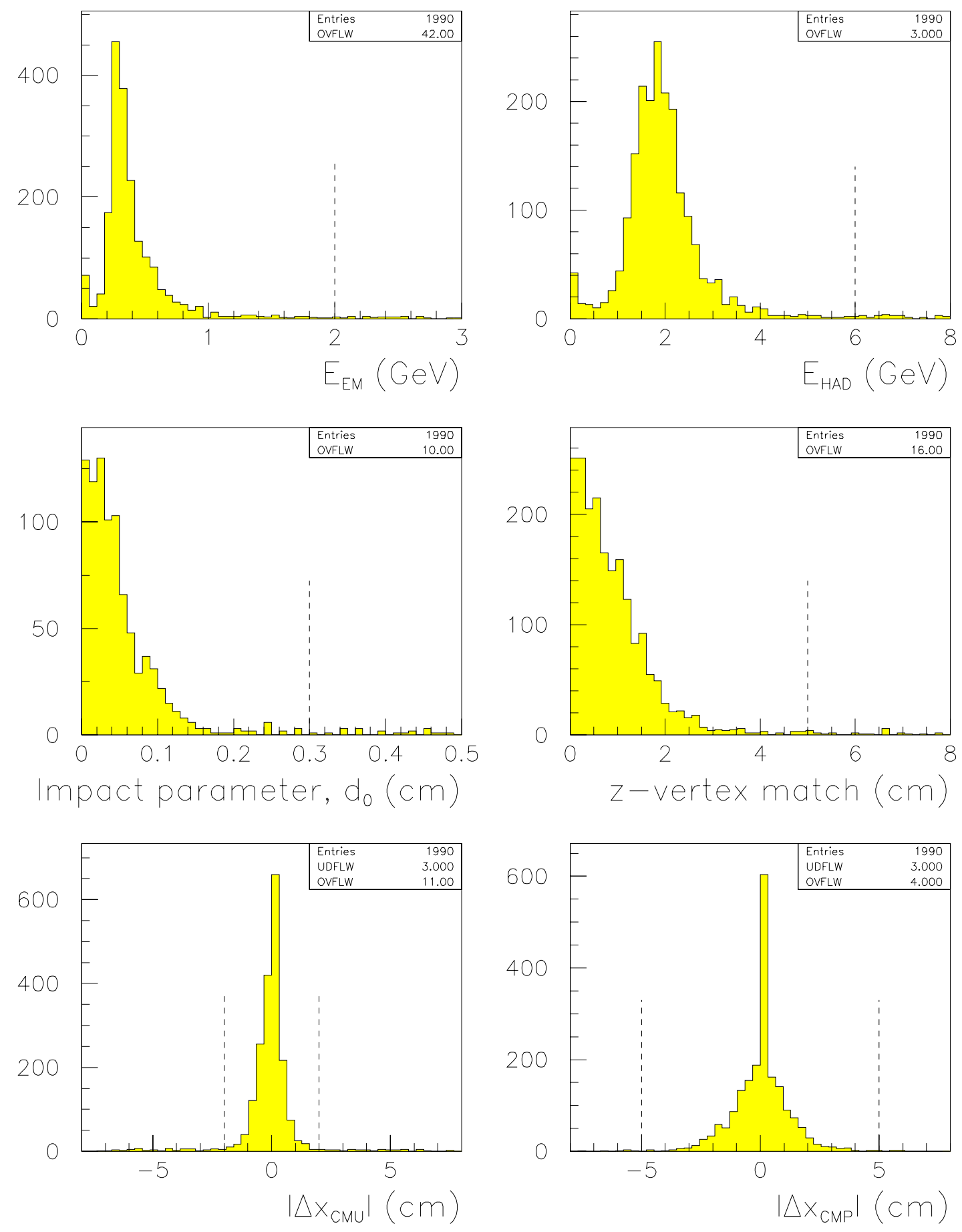

Figure 4.5: Distributions of central muon (CMU, CMP, and CMUP) identification variables, where the dashed line shows where the cut is placed. 

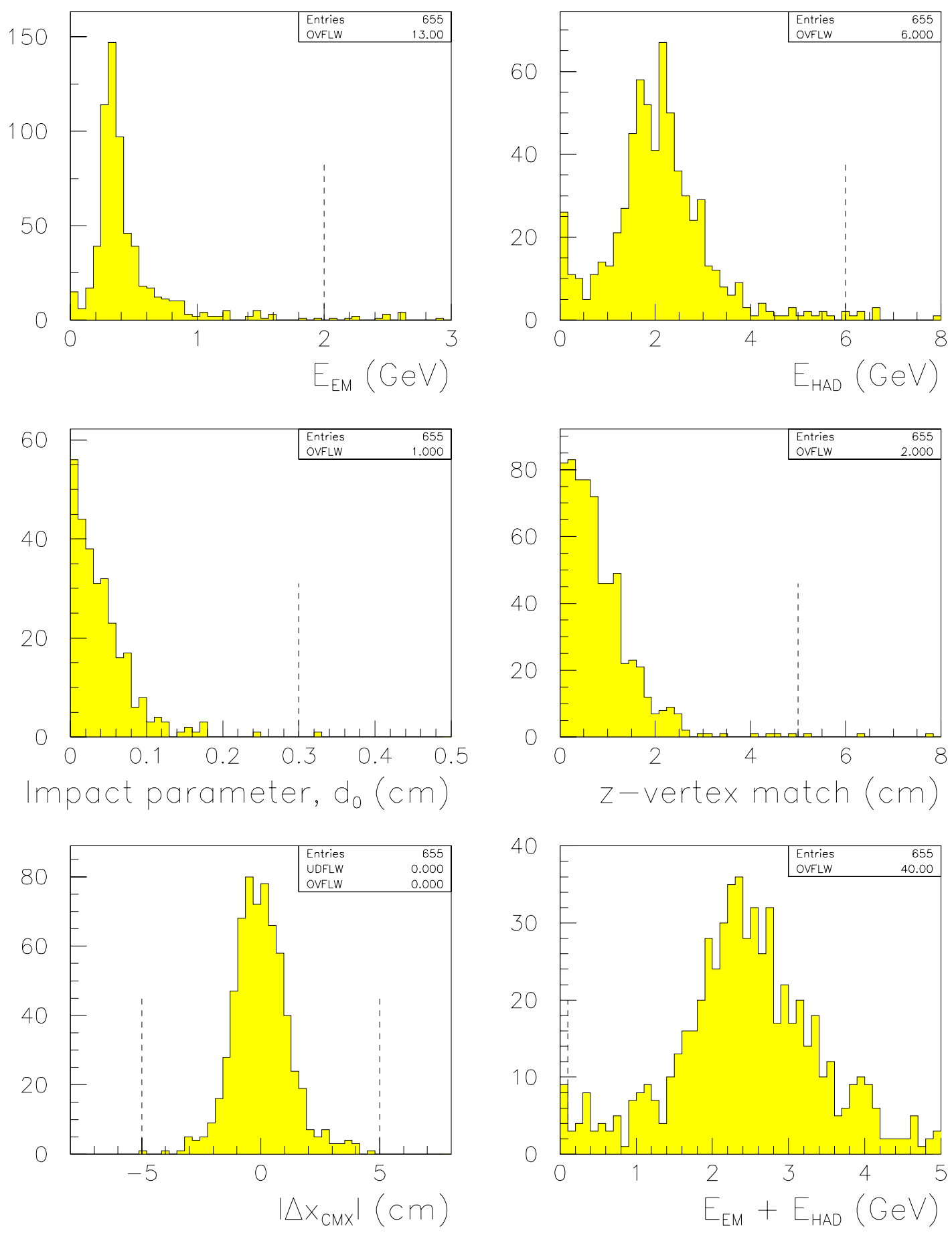

Figure 4.6: Distributions of central muon extension (CMX) identification variables, where the dashed line shows where the cut is placed. 

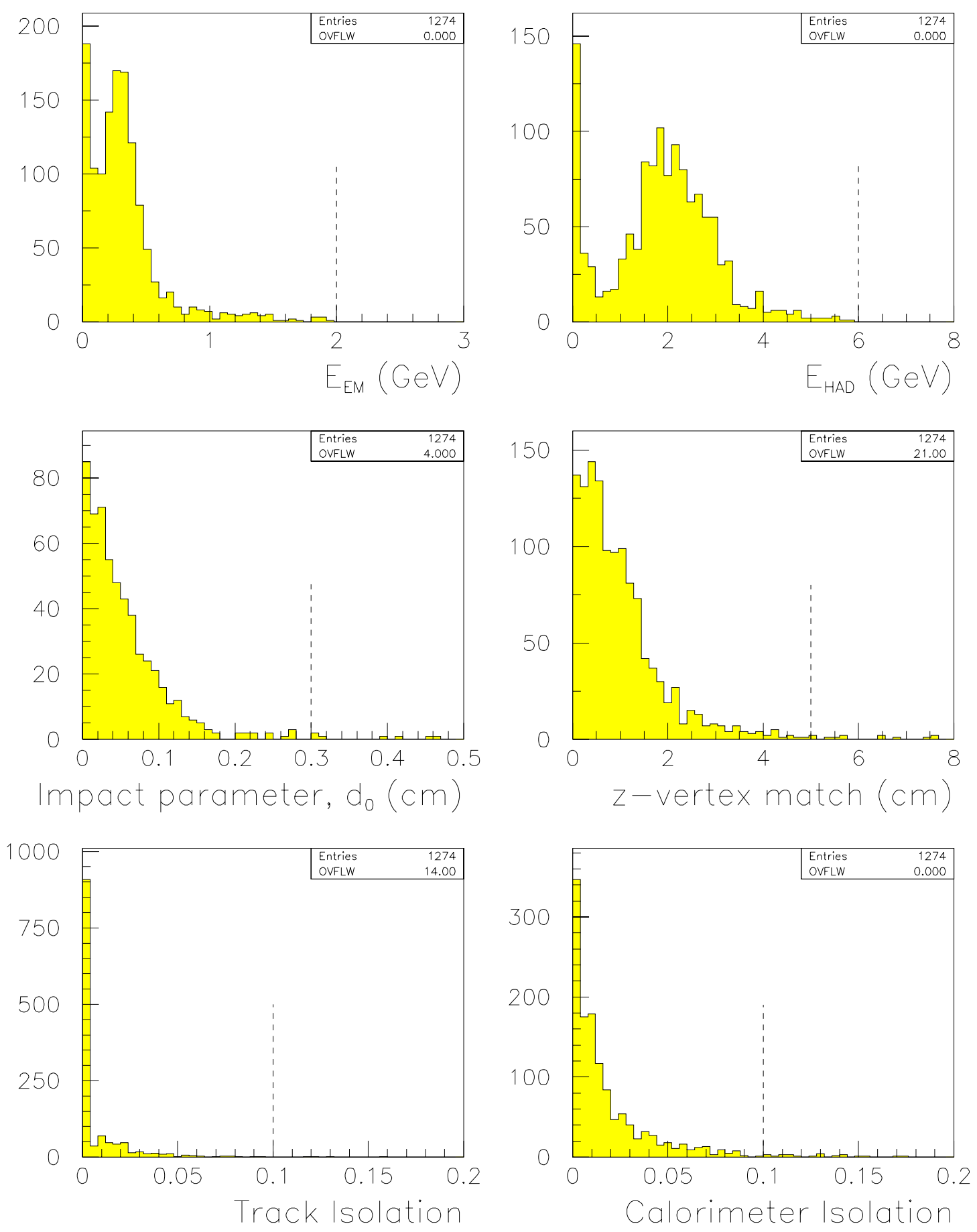

Figure 4.7: Distributions of central minimum ionizing particle (CMI) identification variables, where the dashed line shows where the cut is placed. 
$\left(75 \leq m_{l l} \leq 105 \mathrm{GeV}\right)$, the kinematic variables of the second lepton are shown. The cuts used for each quantity are indicated on the plots.

\subsection{Dilepton categories}

Once all of the high- $p_{T}$ electrons and muons have been identified in an event, they are paired to form dilepton candidates. These come in three general flavors: dielectron $(e e)$, dimuon $(\mu \mu)$, and $e \mu$. We require that at least one lepton in each pair belong to a tight category (TCE, TCM, or CMX), while the other may come from any of the five lepton categories (TCE, LCE, TCM, CMX, or CMI). This gives rise to the 12 distinct tight-tight or tight-loose categories listed in Table 4.4.

Trilepton events are treated separately since very few such events are expected from either $t \bar{t}$ or $\tilde{g} \tilde{g}$ production. Multiple dilepton candidates may be formed from the allowed pairings of three leptons, and each pair is tested separately according to the procedures outlined for dilepton events. For a trilepton event to pass the analysis, at least one pairing must pass all cuts.

\subsubsection{Additional restrictions}

In order for an event to be considered for this analysis, the following conditions must also be met:

- "Good" runs: Occasionally data will be taken under less than optimum running conditions. The accelerator may fail to attain sufficient initial luminosity due to problems during bunch injection, or a particular system within the CDF detector may be performing poorly. Under such circumstances, any data collected during the run is labelled as "bad", and these events are generally not used in physics analyses. All events in our search must come from runs which have been flagged as "good."

- $z$-vertex matching: In the lepton ID cuts for electrons and muons, the $z$-vertex of each lepton is required to be located within $5 \mathrm{~cm}$ of an event-level vertex 


\begin{tabular}{|c|c|c|}
\hline Dilepton type & $\#$ & Dilepton category \\
\hline \multirow[t]{2}{*}{ ee: } & 1 & TCE-TCE \\
\hline & 2 & TCE-LCE \\
\hline \multirow[t]{5}{*}{$\mu \mu:$} & 3 & TCM-TCM \\
\hline & 4 & TCM-CMX \\
\hline & 5 & CMX-CMX \\
\hline & 6 & TCM-CMI \\
\hline & 7 & CMX-CMI \\
\hline \multirow[t]{5}{*}{$\mathrm{e} \mu:$} & 8 & TCE-TCM \\
\hline & 9 & TCE-CMX \\
\hline & 10 & TCE-CMI \\
\hline & 11 & TCM-LCE \\
\hline & 12 & CMX-LCE \\
\hline
\end{tabular}

Table 4.4: There are 12 distinct categories which arise from the pairings of the five classes of leptons (TCE, LCE, TCM, CMX, and CMI), and the requirement that at least one lepton be tight. 
identified by the VTX. For a pair of leptons to be considered a valid dilepton candidate, the event vertex associated with each lepton must match, and the vertex must lie within $|z|<60 \mathrm{~cm}$ of the central interaction region.

- Maximum muon transverse momentum: Any muons identified by the lepton ID cuts (Table 4.2) are required to have $p_{T}<250 \mathrm{GeV} / \mathrm{c}$. This reduces contamination of the sample by cosmic ray events which manage not to confuse the track reconstruction algorithms and appear as large momentum tracks.

Events which contained two or more high- $p_{T}$ leptons but which fail these requirements were not completely removed from consideration by the analysis. Instead, each of these events was evaluated by the filters described in the following sections but was removed from the final results. This procedure facilitates comparison of our results with earlier analyses, especially in light of the fact that the "good run" list has received numerous revisions over time. In the tables of results shown later, these cuts will be folded into the Lepton ID stage, and their effect will not be shown separately, other than in Table 5.1.

\subsection{Cosmic ray removal}

Cosmic rays are elementary particles, originating from both galactic and extragalactic sources, that have been accelerated up to ultra-high energies $\left(>10^{19} \mathrm{eV}\right.$ in some cases). These particles constantly bombard the Earth's atmosphere, where the interaction produces a cascade of elementary particles. Relativistic muons produced in the shower are energetic and long-lived, capable of fully traversing the atmosphere and reaching the surface. If such a muon is able to penetrate the shielding surrounding the CDF detector, it will appear to the event reconstruction software as either a high-momentum track or a pair of back-to-back muons.

Cosmic ray events are often rejected for a variety of reasons. Unlike particles produced by the $p \bar{p}$ collisions at the Tevatron, cosmic rays may appear in the detector at any time. Because they often do not coincide with the beam crossing reported by the accelerator, during which time the detector electronics and readout are active, 
many cosmic rays fail to be detected. When a cosmic ray muon traverses the detector from the outside in, the timing information for hits in the upper half of the detector will be confusing to the tracking algorithms, and even a well-timed event will be discarded. For those cosmics which do enter during the beam crossing and have well-defined tracks, a maximum $p_{T}$ cut applied to all muons at the Lepton ID stage to remove cosmic rays. Cosmic rays events are also be filtered out by searching for muon-like objects which are back-to-back in $\eta$ and $\phi$. This handle may be combined with timing information provided for each hit by the TDC counters. It is possible to check for muon tracks which are "out-of-time" with the overall event or with each other.

The following algorithm is applied to all $\mu \mu$ and $e \mu$ dilepton events. Every high$p_{T}$ muon or minimum ionizing track in a dilepton candidate is systematically paired with all other muon-like objects in the event, and the opening angle (in $\eta$ and $\phi$ ) and timing information for each pair is checked. First, the event is removed if any pair satisfies the tight back-to-back angular cut

$$
\left|\eta_{1}+\eta_{2}\right|<0.1 \text { and }\left|\Delta \phi-180^{\circ}\right|<1.5^{\circ}
$$

A second opening angle cut is combined with timings from the TDCs. For any pair satisfies the relaxed angular cut, the event will be discarded if either muon track fails to coincide with the beam crossing or if the tracks have widely dissimilar TDC times. For any two muon tracks which are loosely back-to-back, i.e.

$$
\left|\eta_{1}+\eta_{2}\right|<0.25 \text { and }\left|\Delta \phi-180^{\circ}\right|<3.0^{\circ}
$$

the event is removed by the cosmic ray filter if either of the following timing conditions is not met:

$$
-.10 \mathrm{~ns}<T D C_{i}<22 \mathrm{~ns} \quad \text { or } \quad|\Delta T D C|<14 \mathrm{~ns} .
$$

The combined effect of these two cuts is to remove about $6.5 \%$ of Run I events identified at the lepton ID stage. Also, $2.5 \%$ of $Z^{0} \rightarrow \mu \mu$ events which would have been identified by the invariant mass cut $\left(75 \leq M_{l l} \leq 105 \mathrm{GeV}\right)$ are removed by the cosmic ray algorithm. 


\subsection{Like-sign/opposite-sign event separation}

After application of the cosmic ray filter, all remaining events are separated according to whether they contain a like-sign or opposite-sign dilepton pair. Trilepton events are assigned to either subsample or both, depending on the relative charges of each pairing among the three or more leptons. The only requirement is that the pairing within the trilepton event must be classifiable according to the allowed tight-tight and tight-loose categories.

\subsection{Isolation}

Leptons from $W$ decay differ from leptons produced by other sources (such as $b$ quark or $\tau$ decay) in that they are typically cleaner and easier to identify. Leptons from semileptonic $b$ decay are often surrounded by hadronic activity from the $b$ jet, and spurious tracks from the jet can interfere with measurement of the lepton ID variables. In order to improve the purity of the dilepton sample, an isolation requirement has been adopted.

The isolation is determined by creating a cone centered about the lepton direction, with fixed size along $\eta$ and $\phi$ of $\Delta R=\sqrt{\Delta \eta^{2}+\Delta \phi^{2}}$. Various choices of cone size are used by CDF: $\Delta R=0.4,0.7,1.0$; in this analysis, $\Delta R=0.4$ is employed. The track isolation is defined as:

$$
I_{t r k}=\frac{p_{T}^{0.4}}{p_{T}}
$$

where $p_{T}^{0.4}$ is the sum of the transverse momenta (other than the lepton $p_{T}$ for any tracks that lie within a cone of radius 0.4 . The calorimeter isolation is defined as:

$$
I_{\text {cal }}=\frac{E_{T}^{04}}{E_{T}} \text { (electrons) } \text { or } I_{c a l}=\frac{E_{T}^{04}}{p_{T}} \quad \text { (muons) }
$$

where $E_{T}^{0.4}$ is the sum of all transverse energy in the calorimeter inside a cone of 0.4 about the lepton direction, again excluding the energy of the lepton itself. In the lepton is a muon, the beam constrained $p_{T}$ is substituted for $E_{T}$, just as with the lepton ID cuts. Distributions of the calorimeter isolation for electrons muons produced by $W, b$, and $\tau$ are shown is Figures 4.8 and 4.9. 

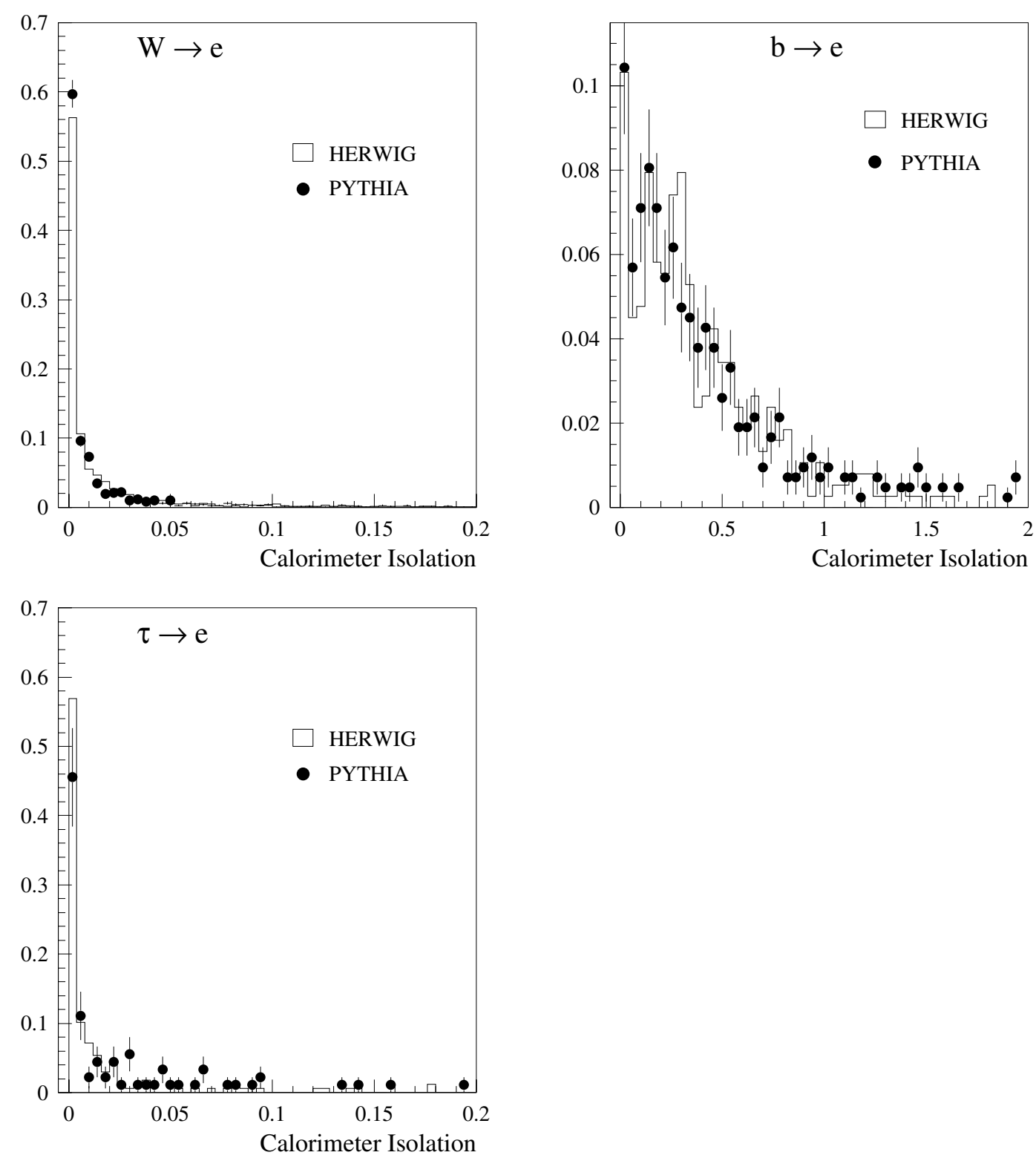

Figure 4.8: Distributions of the calorimeter isolation for electrons originating from three different sources: $W \rightarrow e \nu_{e}, b \rightarrow c e \nu_{e}$, and $\tau \rightarrow e \nu_{e} \nu_{\tau}$. These were produced from $t \bar{t}$ Monte Carlo and only require that the electrons be within the fiducial region of the detector and have $E_{T}>20 \mathrm{GeV}$. 

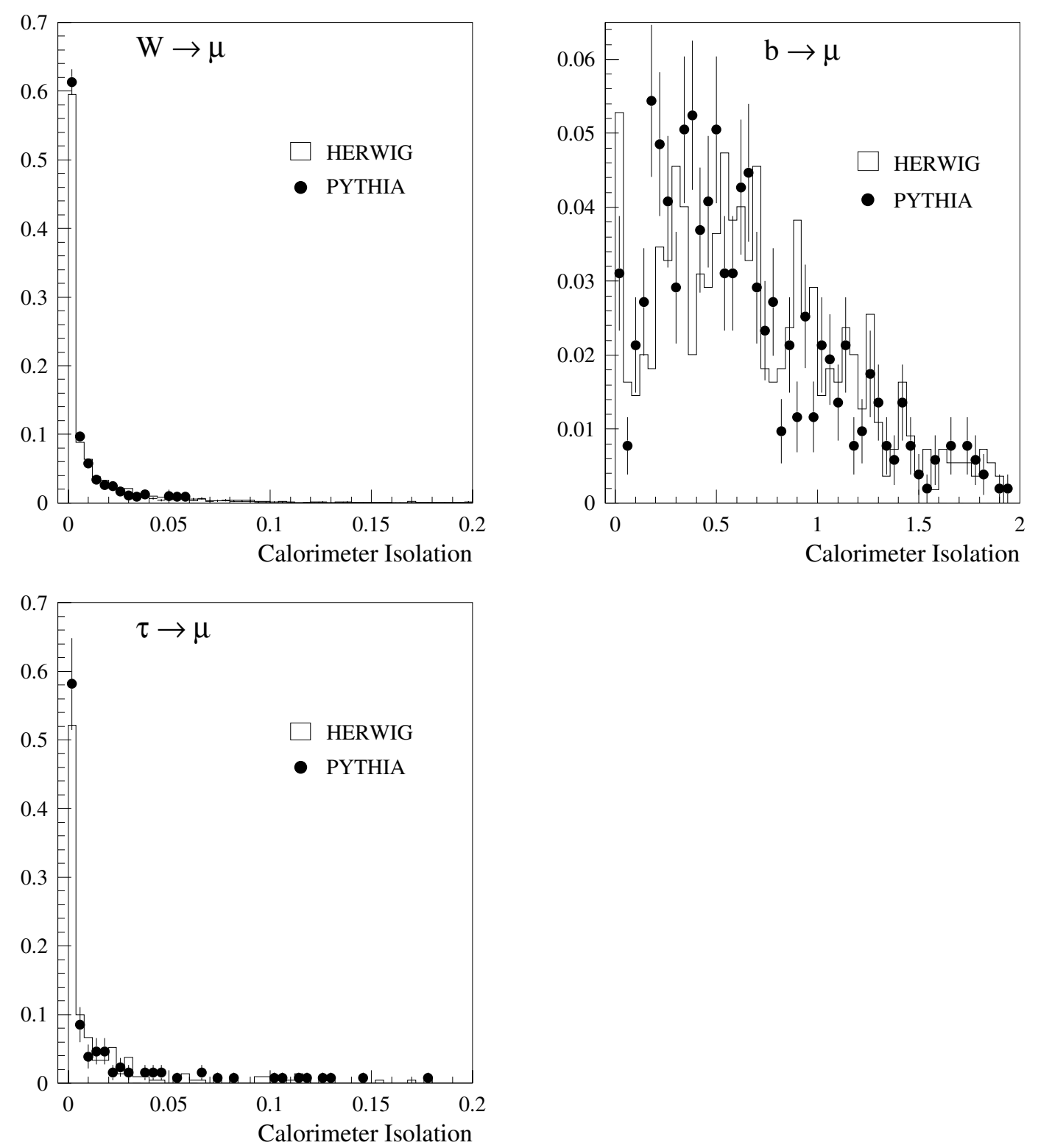

Figure 4.9: Distributions of the calorimeter isolation for muons originating from three different sources: $W \rightarrow \mu \nu_{\mu}, b \rightarrow c \mu \nu_{\mu}$, and $\tau \rightarrow \mu \nu_{\mu} \nu_{\tau}$. These were produced from $t \bar{t}$ Monte Carlo and only require that the muons be within the fiducial region of the detector and have $p_{T}>20 \mathrm{GeV}$. 
At least one tight lepton (TCE, TCM, or CMX) in each event must be isolated in the tracking chamber and calorimeter: $I_{c a l}<0.1$ and $I_{t r k}<0.1$. CMI muons, which are isolated by definition, do not fulfill the isolation requirement. Therefore, both leptons in pair containing a CMI are required to be isolated. In the case of a trilepton event, at least one tight category lepton must be isolated.

The isolation requirement is particularly well suited toward removing $b \bar{b}$ background events. $t \bar{t}$ events are relatively unaffected - the cut is approximately $95 \%$ efficient for top dilepton events. The main loss stems from semileptonic $t \bar{t}$ events where the second lepton is produced by $b$ decay.

\section{7 $Z^{0}$ removal}

In the opposite-sign analysis for top, the major background contribution remaining after same-sign removal and the isolation requirement comes from Drell-Yan events at the $Z^{0}$ resonance. This process $\left(Z^{0} \rightarrow l l\right)$ is discussed in more detail in Section 8.3. In these events, the invariant mass of the dilepton pair should nearly reproduce the $Z$ boson mass. In order to remove by this background, the invariant mass of the dilepton ( $e e$ or $\mu \mu$ only) is required to lie outside the mass window $75 \leq M_{l l} \leq 105$ $\mathrm{GeV}$. For $e \mu$ events, the invariant mass cut is not applied.

The dielectron invariant mass is calculated using:

$$
M_{e e}=\sqrt{\left(E_{1}+E_{2}\right)^{2}-\left(E_{x 1}+E_{x 2}\right)^{2}-\left(E_{y 1}+E_{y 2}\right)^{2}-\left(E_{z 1}+E_{z 2}\right)^{2}}
$$

where the electron energies, $E_{i}$ are measured in the electromagnetic calorimeter. The dimuon invariant mass is determined by:

$$
M_{\mu \mu}=\sqrt{\left(P_{1}+P_{2}\right)^{2}-\left(P_{x 1}+P_{x 2}\right)^{2}-\left(P_{y 1}+P_{y 2}\right)^{2}-\left(P_{z 1}+P_{z 2}\right)^{2}} .
$$

where the muon momenta, $P_{i}$, are taken from the beam-constrained tracks in the CTC. To illustrate the effect of the $Z^{0}$ mass filter, distributions of the invariant mass are shown for electrons in Figure 4.10 and for muons in Figure 4.11. These are opposite-sign dilepton events from Run I data which have passed the lepton ID, cosmic ray, and isolation cuts. $89.3 \%(2425 / 2716)$ of dielectron events and 89.0\% (2789/3133) 

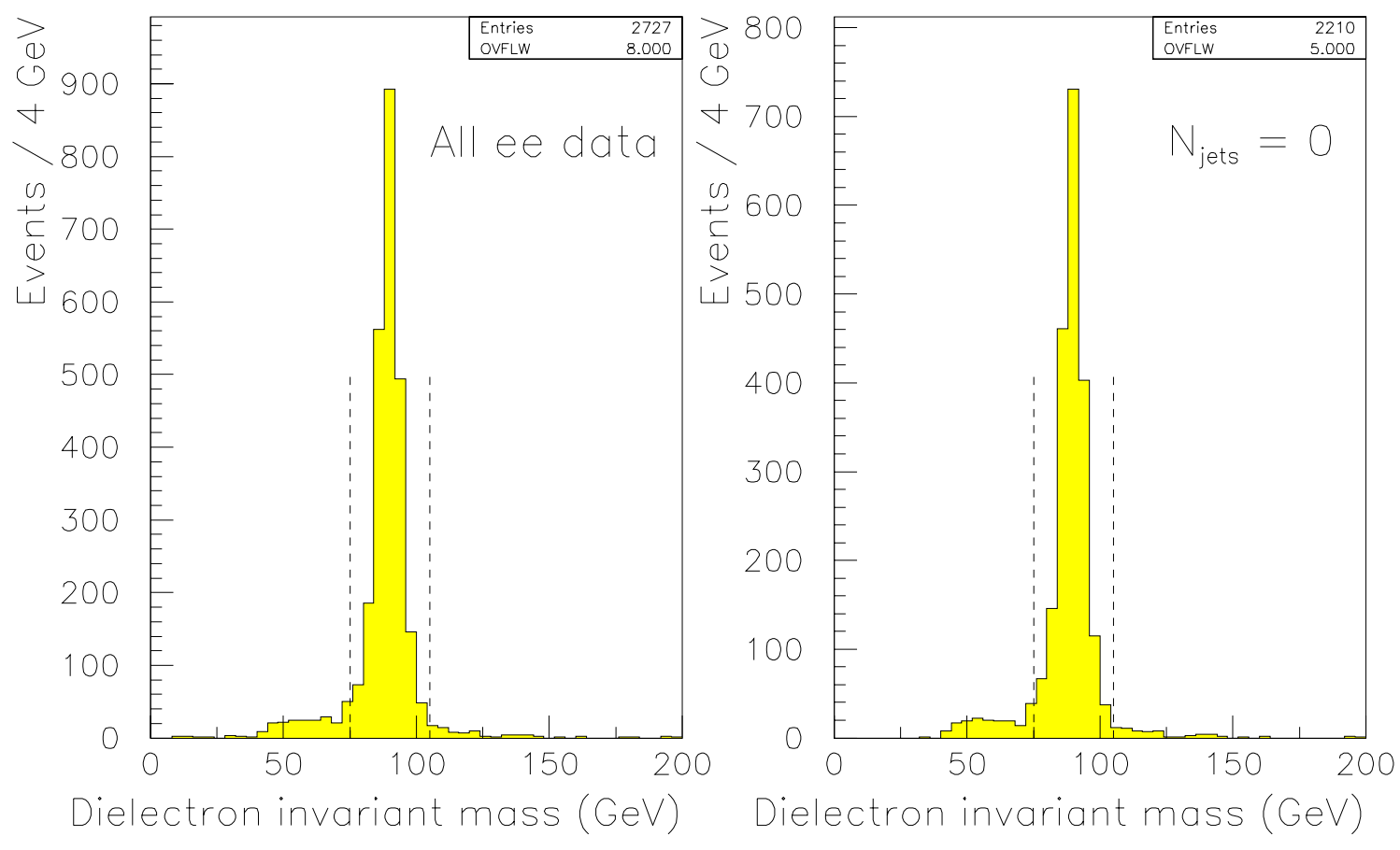

Dielectron invariant mass (GeV)
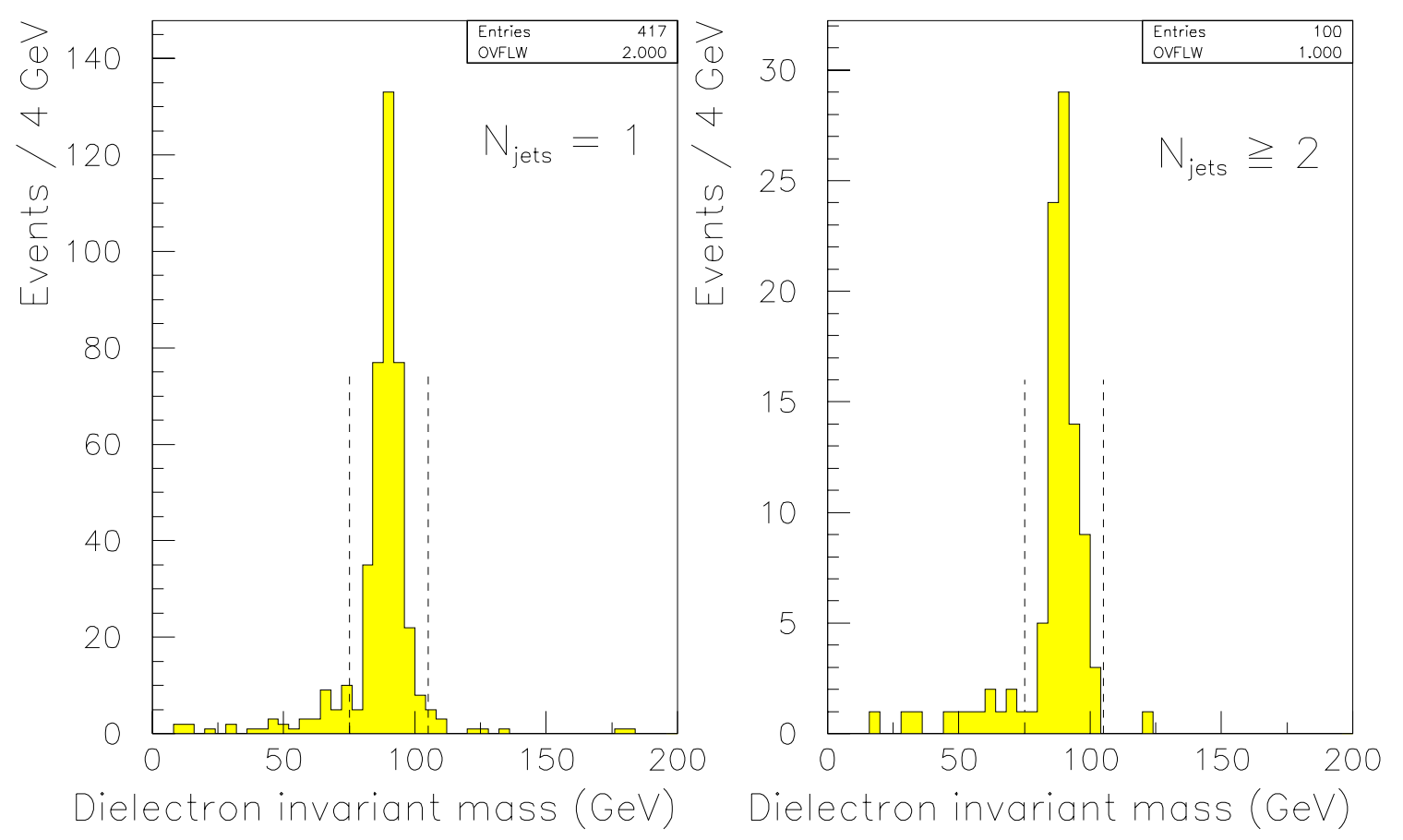

Figure 4.10: Distributions of the invariant mass for dielectron events in Run I data. These are shown for opposite-sign events which pass the lepton ID, cosmic ray, and isolation cuts. The dashed lines show the range of masses removed by the $Z^{0}$ mass cut. 

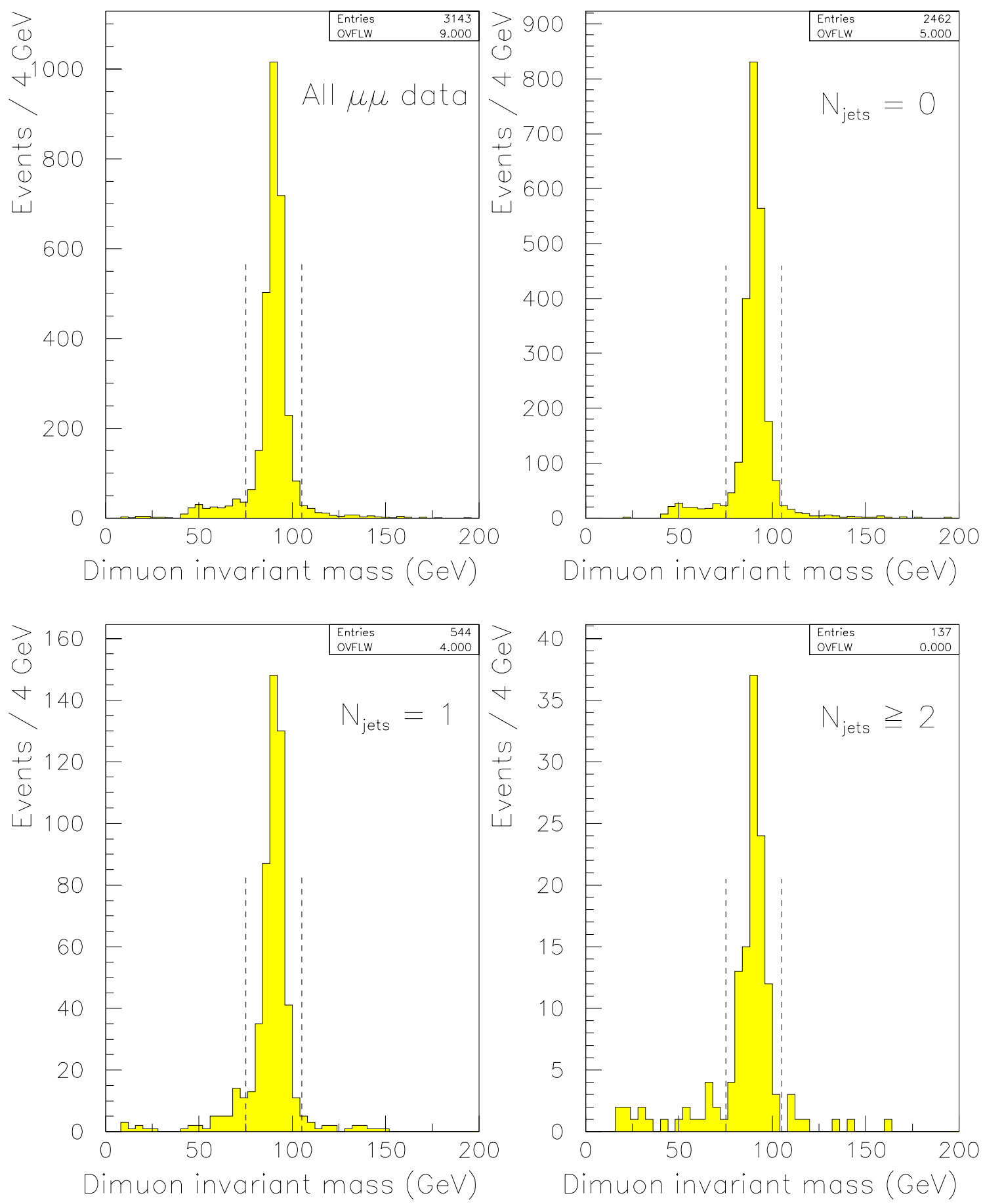

Figure 4.11: Distributions of the invariant mass for dimuon events in Run I data. These are shown for opposite-sign events which pass the lepton ID, cosmic ray, and isolation cuts. The dashed lines show the range of masses removed by the $Z^{0}$ mass cut. 
of dimuon events are removed at this stage. (Once all $e \mu$ and trilepton events are included, $88.4 \%(5215 / 5898)$ of events passing the isolation stage are filtered by the invariant mass cut.)

Near the end of Run I, an additional $Z^{0}$ removal routine was developed to handle radiative $Z^{0}$ events [29], where the $Z^{0}$ boson radiates a photon. (Actually, one of the electrically charged decay products of the $Z^{0}$ radiates the photon.) This process was not expected to contribute significantly to the signal $(<0.1$ events expected in Run I), yet one event (58281/44805, the " $\mu \mu \gamma$ " event) which passes the opposite-sign top dilepton analysis appears to come from this source.

Radiated photons appear as electromagnetic energy in the calorimeter without a corresponding track in the CTC. A set of identification cuts have been developed for photons, and these are shown in Table 4.5. For any event which contains a $10 \mathrm{GeV}$ photon, the invariant mass of the dilepton + photon is calculated, and the event is removed if that mass falls within the previously defined $Z$ window: $75 \leq M_{l l \gamma} \leq 105$ $\mathrm{GeV}$.

In our Run I dilepton sample, there are 21 opposite-sign dilepton + photon events (11 dielectron + photon and 10 dimuon + photon $)$. In Figure 4.12, we have plotted the invariant mass of the dilepton plus photon for these events. All but 1 dielectron event and 1 dimuon event fall within the $Z$ mass window and are removed by the radiative $Z$ cut.

We do not anticipate that there will be a significant contribution to the same-sign channel from Drell-Yan $Z^{0}$ or radiative $Z^{0}$ events, yet we retain the cut anyway. These processes do not directly lead to final states with same-sign dileptons. However, the possibility exists that some opposite-sign events may appear to be like-sign events because of charge mismeasurement due to tracking problems. (This is more likely for muons than electrons.) The $Z^{0}$ removal algorithm, as it is currently implemented, does not use any charge information when performing the invariant mass cuts. 


\begin{tabular}{|cc|}
\hline \hline Variable & Cut \\
\hline Fiduciality & Central (Det=0) EM cluster \\
$E_{t}$ & $\geq 10 \mathrm{GeV}$ \\
\# of 3-D tracks pointing to EM cluster & 0 \\
CES $\chi_{\text {avg }}^{2}=\frac{\chi_{\text {strip }}^{2}+\chi_{\text {wire }}^{2}}{2}$ & $<20$ \\
$E_{H A D} / E_{E M}$ & $<0.05+\frac{0.045 \mathrm{xE}}{100}$ \\
$L_{s h r}$ & $<0.2$ \\
$\Sigma P_{t}^{0.4}$ & $<2 \mathrm{GeV}$ \\
$I_{\text {cal }}$ & $<0.15$ \\
\hline \hline
\end{tabular}

Table 4.5: Photon identification requirements for the radiative $Z^{0}$ cut. 

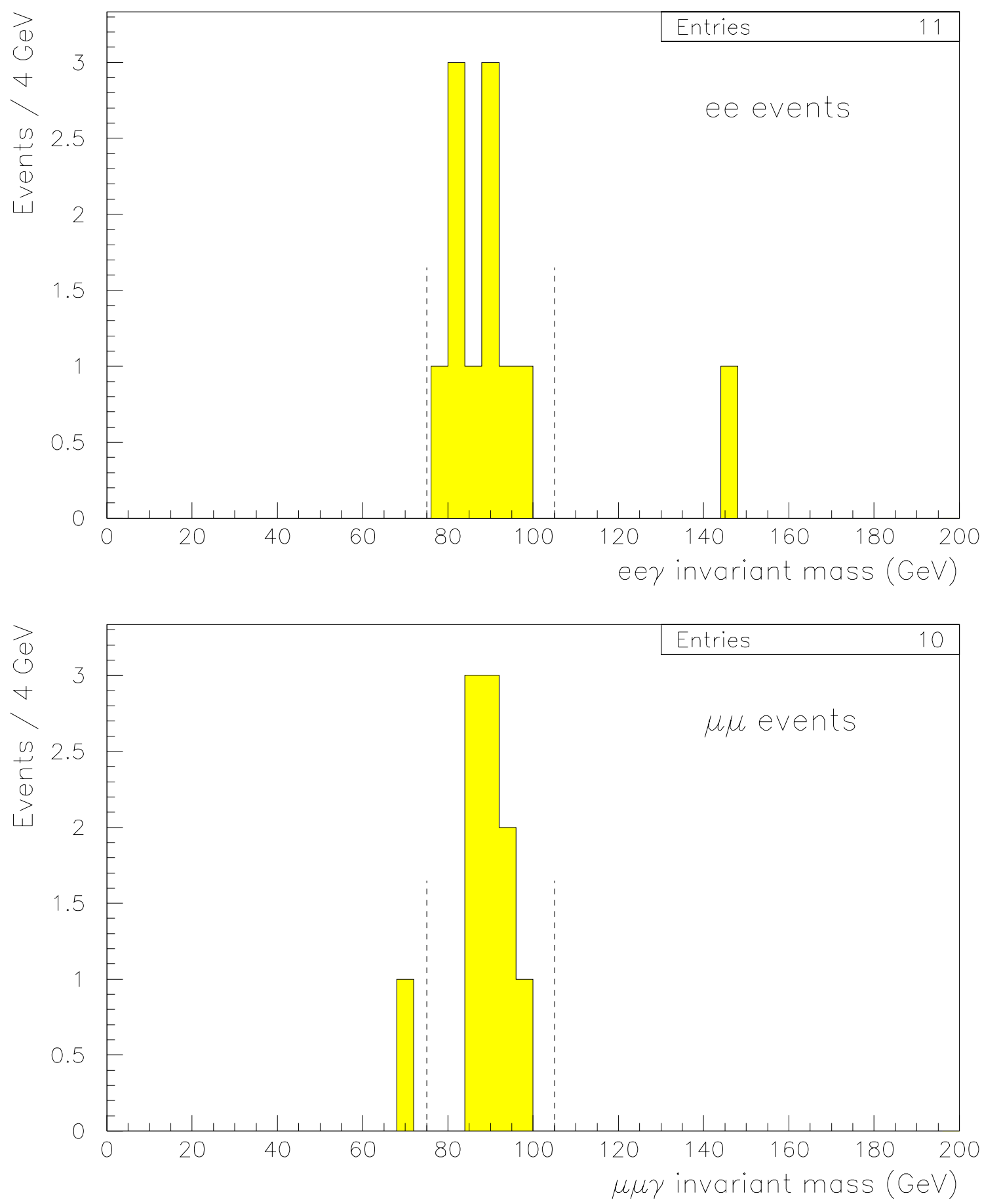

Figure 4.12: Distributions of the photon + dilepton invariant mass for $e e$ and $\mu \mu$ events in Run I data. These are shown for opposite-sign events which pass the lepton ID, cosmic ray, isolation, and invariant mass cuts. The dashed lines show the range of masses removed by the radiative $Z^{0}$ mass cut. 


\subsection{Missing energy}

In $t \bar{t}$ production, the neutrinos from $W$ decay escape the detector completely and carry energy and momentum away from the event. Gluino events $(\tilde{g} \rightarrow t \overline{\tilde{t}})$ add another source of missing energy, as the neutrinos are joined by neutralinos from stop decay $\left(\tilde{t} \longrightarrow c \tilde{\chi}_{1}^{0}\right)$. As mentioned in Section 3.2.1, the resulting energy imbalance in the plane perpendicular to the beam axis offers an avenue for indirectly detecting such particles. After summing up all of the transverse energy and momenta in an event, a large remainder hints at the presence of neutrinos or other weakly interacting particles, such as the neutralino.

The raw missing $E_{T}$ is calculated by attributing a vector to each cluster of energy deposited in the electromagnetic and hadronic calorimeters. The magnitude and direction is summed up over all clusters in the event, and the result is negated to obtain the vector for the missing energy. One correction to the raw $\mathbb{E}_{T}$ is made for muons. The defining characteristic of the muon is the well-defined, high- $p_{T}$ track, not the cluster energy. So for any TCM, CMX, or CMI muons identified in an event, the calorimeter energy is replaced by the beam-constrained transverse momentum. The difference between the $\mathrm{x}$ - and $\mathrm{y}$-components of the muon $p_{T}$ and the corresponding components of the EM and hadronic calorimeter energy is subtracted from the missing energy:

$$
\begin{aligned}
& \mathscr{E}_{T, x}=\mathscr{E}_{T, x}-\left(P_{x}-\left(E_{E M}+E_{H A D}\right) \sin \theta \cos \phi\right) \\
& \mathbb{E}_{T, y}=\mathbb{E}_{T, y}-\left(P_{y}-\left(E_{E M}+E_{H A D}\right) \sin \theta \sin \phi\right)
\end{aligned}
$$

Another adjustment accounts for the fact that jet energies (see Section 4.9) are corrected for detector effects. The difference between uncorrected and corrected jet energies is factored back into the $\mathbb{E}_{T}$ calculation.

Mismeasurement of a lepton or jet energy is also a potential source of missing $E_{T}$. In Drell-Yan events, which have no neutrinos, the missing energy tends to align with one of the jets. In $Z^{0} \rightarrow \tau^{+} \tau^{-}$events, the missing energy will point close to one of the leptons. So for small $\mathbb{E}_{T}$, one should ensure that the missing energy direction is not correlated with any leptons or jets in the event. By restricting the allowed opening angle between the missing energy and any nearby leptons or jets, contributions from 
these background sources are reduced.

Events are rejected if the corrected missing energy satisfies either:

- $\mathbb{E}_{T}^{\text {corr }}<25 \mathrm{GeV}$, or

- $\left|\Delta \phi\left(\mathbb{E}_{T}, l\right)\right|<20^{\circ}$ or $\left|\Delta \phi\left(\mathbb{E}_{T}, j\right)\right|<20^{\circ}$ for any leptons $(l)$ passing the lepton ID requirements or any jets $(j)$ passing the jet ID cuts (defined in Section 4.9). This cut only applies for missing energies in the range $25 \leq \mathbb{E}_{T}^{c o r r} \leq 50 \mathrm{GeV}$.

\subsection{Jet identification}

When quarks and/or gluons are produced in an event, the strong force between proximate colored objects leads to fragmentation. The binding energy allows for creation of quark-antiquark pairs, and a variety of mesons and baryons are formed as the quarks coalesce into colorless states. A shower of charged and neutral particles emerges, carrying the net momentum of the original object. Jets formed by the hadronization of energetic quarks are collimated and deposit most of their energy in a localized portion of the detector.

At CDF, a jet is identified by a cluster of energy deposition in the calorimeter. Recall that the calorimeter towers are segmented in $\eta-\phi$ and project back to the interaction point. Towers with the highest energy are selected as seed towers, and the total amount of energy deposited in towers within a cone about the seed tower provides an estimate of the original particle energy. The cone size is defined in $\eta$ and $\phi$ by $R \equiv \sqrt{\eta^{2}+\phi^{2}}$, and the clustering algorithm allows for cone sizes of $R=0.4$, $0.7,1.0$. The particular cone radius used must be large enough to encompass the jet yet not so large that energy from other object is incorrectly clustered with the jet. The jet clustering algorithm is fully described in Ref. [30].

For the dilepton analysis, two or more jets are required, where a "jet" is defined as follows:

- Raw transverse energy: $E_{T}^{\text {raw }}>10 \mathrm{GeV}$

- Detector eta: $\left|\eta_{\text {det }}\right|<2.0$ 
- Any jet must not be located within a cone of $0.4\left(\Delta R=\sqrt{\Delta \eta^{2}+\Delta \phi^{2}}\right)$ around an energy cluster identified as a TCE or LCE electron

The raw transverse energy is the sum of all the EM calorimeter tower energies within a cone of $R=0.4$. The raw $E_{T}$ tends to be an underestimate of the true parton energy and suffers from several inefficiencies:

- Non-uniform detector response along calorimeter boundary regions, tower-bytower variations in detector response, and understanding of the overall detector response

- Energy outside the cone which is not included in the cluster (out-of-cone losses)

- Muons and neutrinos produced in the jet but which deposit little energy in the EM calorimeter

- Shower particles with insufficient momentum to escape the solenoid and enter the calorimetry

- Underlying event processes which contribute energy within the jet cone

Monte Carlo studies [30, 31] have developed standard methods to correct for detector effects, out-of-cone losses, and underlying event contributions. In this analysis, only the foremost is applied to arrive at the corrected jet energy, $E_{T}^{c o r r}$. Corrections may add up to $30 \%$. Figure 4.13 shows the difference between raw and corrected energies for the two highest $E_{T}^{\text {raw }}$ jets for Run I opposite-sign events which have passed the lepton ID and isolation stages.

The difference between raw and corrected energies is used to correct the missing energy calculation. Note that in the $\mathbb{E}_{T}$ case, jets in the pseudorapidity range $|\eta|<2.4$ are considered, which slightly differs from the jet ID geometrical acceptance.

\subsection{Summary}

A short summary of all the event requirements to be used in the same-sign dilepton analysis is provided in Table 4.6. In the chapters which follow, we shall present the 

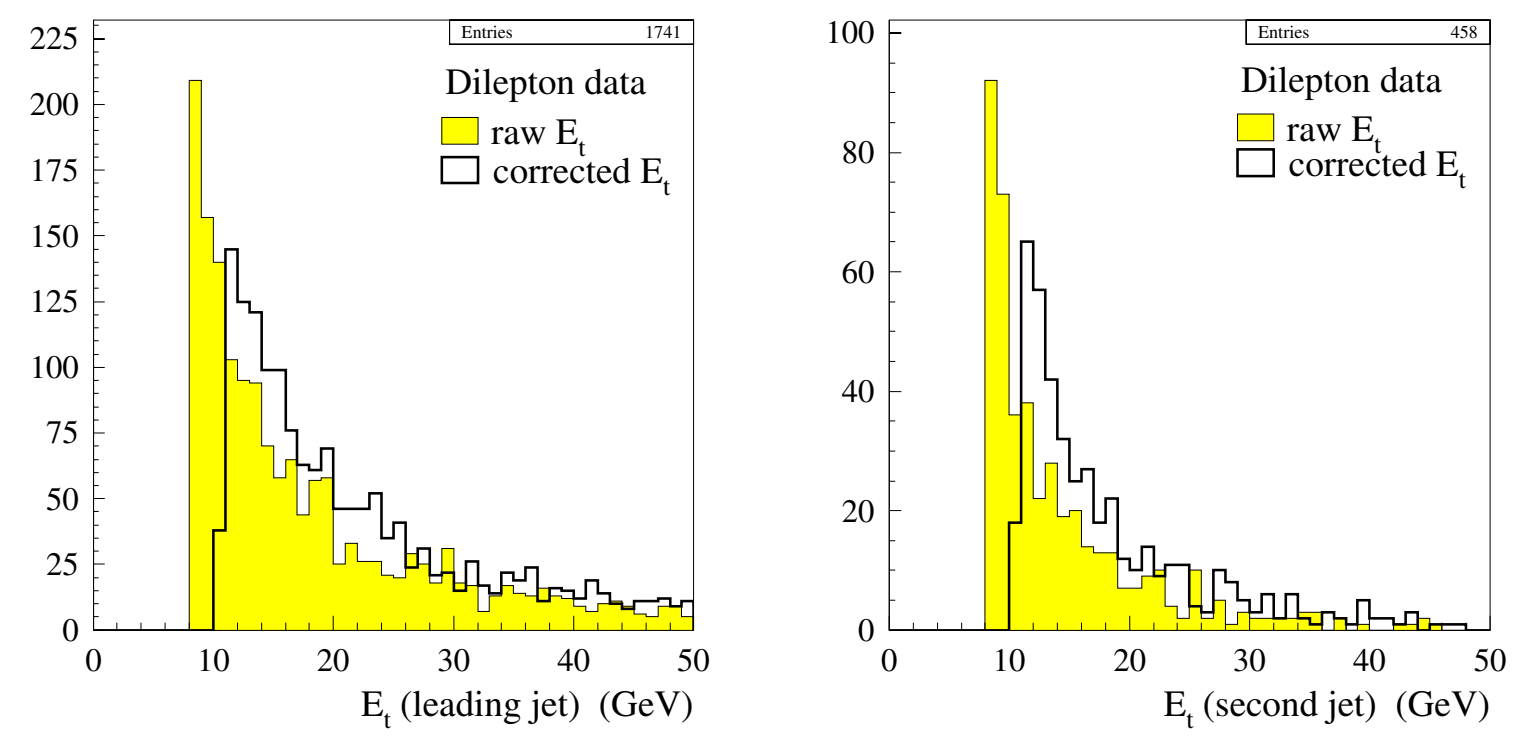

Figure 4.13: Comparison of the raw and corrected transverse energy for the highest $E_{T}^{\text {raw }}$ and second highest $E_{T}^{\text {raw }}$ jets, for opposite-sign dilepton events which pass the lepton ID and isolation cuts.

results of applying these analysis techniques to the data collected by CDF during Run I, to Monte Carlo simulations of $\tilde{g} \tilde{g}$ production and decay via $\tilde{g} \rightarrow t \overline{\tilde{t}}$, and to Monte Carlo samples of all the background processes which are expected to contribute to the same-sign dilepton channel. 


\begin{tabular}{|c|c|c|}
\hline Cut \# & Description & More information \\
\hline 0 & Lepton ID & $\begin{array}{l}\text { identify electron, muon, and } \\
\text { min. ionizing objects }\end{array}$ \\
\hline 1 & Cosmic ray filter & $\begin{array}{l}\text { remove back-to-back, out-of- } \\
\text { time } \mu \mu\end{array}$ \\
\hline 2 & Opposite/Same sign cut & \\
\hline 3 & Isolation cut & $\begin{array}{l}\text { at least } 1 \text { tight lepton with } \\
I_{\text {track }}, I_{\text {cal }}<0.1\end{array}$ \\
\hline 4 & $\mathrm{Z}$ mass cut & veto $75 \leq M_{l l} \leq 105 \mathrm{GeV}$ \\
\hline 5 & Missing energy & $\mathscr{E}_{T}{ }^{\text {corr }}>25 \mathrm{GeV}$ \\
\hline 6 & $\Delta \phi\left(\mathscr{E}_{T}, l\right)>20^{\circ}$ & only if $25<\mathbb{E}_{T}<50$ \\
\hline 7 & $\Delta \phi\left(\not_{T}, j\right)>20^{\circ}$ & only if $25<E_{T}<50$ \\
\hline 8 & Number of jets $\geq 2$ & $E_{t}^{\text {raw }}>10 \mathrm{GeV},\left|\eta_{\text {det }}\right|<2.0$ \\
\hline
\end{tabular}

Table 4.6: Summary of all selection requirements which will be applied to Run I data and Monte Carlo events in the same-sign dilepton analysis. 


\section{Chapter 5}

\section{Data Results}

In this chapter, we discuss the results of applying the previously described event selection criteria to the data collected during Run I. We begin with a description of the inclusive electron and muon datasets which were chosen as the initial starting point for the analysis. Then we describe the results of what are effectively two separate analyses. The first involves the selection of opposite-sign dilepton pairs and serves as a consistency check by allowing us to compare our techniques against those used in the original search for the top quark in the opposite-sign dilepton channel. Once we confirm that we have accurately reproduced those results to within a reasonable approximation, we shall proceed to the results of the same-sign analysis of the data, in search of evidence for gluino production at the Tevatron..

\subsection{Datasets}

The full Run I dataset contains millions of recorded events and resides on hundreds of magnetic tapes located in a robotic storage vault. Very rarely should an analysis involve processing the entire dataset, as this would be extremely time-consuming and undesirable. Instead, the typical starting point is a subsample of more manageable size which only contains events selected because they include a particular class of object (high- $p_{T}$ electrons, for instance) and/or pass a loose set of requirements on the event topology. Many subsamples consist of events which enter via a particular 
trigger path or have been constructed as a custom sample for one of the analysis groups. The main caveat is that the initial sample should be chosen such that none of the selection criteria used to create the sample are more restrictive than those one intends to use in the analysis. Otherwise, there may exist events in the full dataset which would have passed the analysis but were removed from consideration by selecting an inappropriate subsample.

For this analysis, we have chosen datasets which are primarily based upon the Level 3 inclusive electron and inclusive muon triggers introduced in Section 3.8. Separate electron and muon datasets were created, where the foremost condition for each event was the presence of a well-defined $e$ or $\mu$ candidate. The selection criteria used to produce the electron and muon samples differs slightly for Run Ia and Run Ib, so we describe them separately.

The Run Ia dataset we use is a custom subset of the inclusive lepton samples produced by Level 3 [32]. The selection criteria for the electron sample were:

- central electrons only (no forward or plug electrons)

- $E_{T}>18 \mathrm{GeV}$

- $p_{T}>13 \mathrm{GeV} / \mathrm{c}$

- $E_{H A D} / E_{E M}<0.125$

- $L_{s h r}<0.2$

- Track-CES matching: $|\Delta x|<3 \mathrm{~cm}$

- Track-CES matching: $|\Delta z|<5 \mathrm{~cm}$, and

- $\chi_{\text {strip }}^{2}<10.0$.

The selection criteria for the muon sample were:

- $p_{T}>15 \mathrm{GeV} / \mathrm{c}$ (no beam constraining performed)

- $E_{H A D}<6 \mathrm{GeV}$ 
- no $E_{E M}$ requirement

- Track-stub matching: $|\Delta x|<10 \mathrm{~cm}$ (for CMU only and CMU/CMP)

- Track-stub matching: $|\Delta x|<20 \mathrm{~cm}$ (for CMP only), and

- Track-stub matching: $|\Delta x|<20 \mathrm{~cm}$ (for CMX).

The electron dataset contains 133,805 events, and the muon dataset contains 83,051 events. There are 529 duplicate events which appear in both sets. This occurs because the electron and muon samples are created independently, and events which contain both a high- $p_{T}$ electron and a high- $p_{T}$ muon will be selected for both samples. Once duplicate events have been removed, we perform the first stage of the analysis by applying the Lepton ID requirements. The number of events containing two or more high- $p_{T}$ lepton candidates is 1,214 . The results of subsequently applying the "good run," matching z-vertex, and maximum muon momentum requirements are shown in Table 5.1. The final number of Run Ia events which pass the Lepton ID stage is 1,011 .

For the Run Ib sample, we use a sample that originated with the Level 3 trigger for "Stream A" high- $p_{T}$ inclusive electrons and muons. At Level 3, the muon requirements were straightforward:

- $p_{T}>18 \mathrm{GeV} / \mathrm{c}$

- $E_{H A D}<6 \mathrm{GeV}$

- no $E_{E M}$ requirement

- Track-stub matching: $|\Delta x|<5 \mathrm{~cm}(\mathrm{CMU})$

- Track-stub matching: $|\Delta x|<10 \mathrm{~cm}(\mathrm{CMP})$, and

- Track-stub matching: $|\Delta x|<10 \mathrm{~cm}$ (CMX).

The electron requirements were more complicated since high momentum electrons could enter through one of five trigger paths, all of which are listed in Table 5.2. Note 


\begin{tabular}{|l|c|c|}
\hline \hline Lepton ID requirement & Run Ia & Run Ib \\
\hline Inclusive electron dataset & 133,805 & 128,761 \\
Inclusive muon dataset & 83,051 & 86,901 \\
Duplicate events & 529 & 42 \\
\hline Inclusive electron or muon dataset & 216,327 & 215,620 \\
\hline \hline Two or more high- $p_{T}$ leptons & 1,214 & 5,586 \\
Event satisfies "good run" requirement & 1,023 & 5,339 \\
Leptons have matching $z$-vertices & 1,016 & 5,258 \\
$p_{T}<250$ GeV/c for all muons & 1,011 & 5,245 \\
\hline Total \# of events which pass Lepton ID stage & & 6,256 \\
\hline \hline
\end{tabular}

Table 5.1: Number of events in the Run Ia and Run Ib inclusive electron and muon datasets. Also shown are the number of events which pass each of the requirements comprising the Lepton ID stage of the analysis.

that the "tight cuts" trigger is the same as that used for the Run Ia inclusive electron sample.

The final Run Ib inclusive electron and inclusive muon datasets that we have chosen were obtained by processing the Stream A samples using the same requirements as those for Lepton $\operatorname{ID}[33,34]$. In the case of the inclusive electron sample, events were chosen from Stream A if at least one electron passed the fiducial requirements (Section 4.1.1), the loose set of quality cuts (Table 4.1), and the conversion removal algorithm (Section 4.1.3). This reduced the sample size from approximately 750,000 Stream A electron events to 128,761 events. The inclusive muon sample was obtained in the same way, by requiring the presence of a muon which passes the set of quality cuts given in Table 4.2. Out of 570,000 Stream A muon events, 86,901 were selected 


\begin{tabular}{|c|c|}
\hline \hline \multirow{2}{*}{ Tight cuts } & $E_{T}>18 \mathrm{GeV}, p_{T}>13 \mathrm{GeV} / \mathrm{c}, L_{s h r}<0.2$, \\
& $E_{H A D} / E_{E M}<0.125,|\Delta x|<3 \mathrm{~cm},|\Delta z|<5 \mathrm{~cm}$, \\
\hline Central $W$ boson & $\chi_{\text {strip }}^{2}<10$ \\
\hline Tight $W$, no track & $E_{T}>22 \mathrm{GeV}, p_{T}>13 \mathrm{GeV} / \mathrm{c}$, \\
& $E_{H A D} / E_{E M}<0.125, E_{T}>22 \mathrm{GeV}$ \\
\hline$Z^{0} \rightarrow e e$ & $E_{\text {border }}<2.5 \mathrm{GeV}, E_{T}>25 \mathrm{GeV}, \chi_{\text {strip }}^{2}<20$ \\
\hline High $E_{T}$ & Electron $1: E_{T}>18 \mathrm{GeV}, p_{T}>13 \mathrm{GeV} / \mathrm{c}$ \\
\hline \hline
\end{tabular}

Table 5.2: Multiple trigger paths at Level 3 for high- $p_{T}$ electrons. An electron is included in the Stream A inclusive electron sample if selected by any of the above triggers.

for our inclusive muon sample.

There are 42 events shared by the two inclusive lepton samples. After these are removed, the Run Ib dataset contains 215,620 events. Just as before, we apply the Lepton ID requirements of our analysis, including those of Section 4.3.1. The results are summarized in Table 5.1. This reduces the sample down to 5,586 events containing two or more high- $p_{T}$ leptons and 5,245 events which completely pass the Lepton ID stage.

Therefore, the total number of Run I events which contain two or more leptons and pass the lepton ID stage of our analysis is 6,256 . These events form the basis of the opposite-sign and like-sign analyses which follow. 


\section{$5.2 \quad$ Opposite-sign analysis}

First, we perform an analysis focusing on the opposite-sign dilepton events in the Run I datasets. The CDF analysis control software is a powerful, yet complex, package of algorithms and utilities, including the TOPFND library designed by the top quark analysis group for studying $t \bar{t}$ events. By comparing our results with the findings of the original top dilepton analysis [28], we may assure ourselves that we have properly designed our analysis module and that we have incorporated all of the subtleties of the original analysis.

The results of the opposite-sign analysis of the data are shown in Table 5.3. The number of events which pass each of the selection criteria are further divided according to dilepton category. Of the 6,256 events which contain a dilepton candidate at the Lepton ID stage, only 9 events (including 1 trilepton event) pass all stages of the analysis.

One should remember that the top dilepton analysis being compared to here is several years old, and the CDF code has undergone many small "improvements" in the intervening time. As multiple studies of the data are performed, some runs may be reassigned to either the "good run" or "bad run" list, causing events to be added or removed from an analysis. Exact reproduction of some results is near impossible, since changes in the alignment geometry or calibration constants affect the calculation of kinematic variables, which will alter whether certain events near the boundary of a selection cut will pass or fail.

Given these precautions, our results compare quite favorably with the top dilepton analysis. The numbers of events passing each of the cuts up through the missing energy requirement are within the expected variation considered the potential changes listed above. The 9 signal events, including the 1 trilepton event, have been exactly reproduced.

Differences in the 0- and 1-jet bins (after all other cuts have been applied) are at the few-event level, as shown in Table 5.4. The differences are limited to three events: one TCM-CMI event and two TCM-TCM events. Although none of these events would have passed the complete analysis due to the 2-jet cut, they have been examined 


\begin{tabular}{|c|c|c|c|c|c|c|c|c|c|c|c|}
\hline \multirow[b]{3}{*}{ Category } & \multicolumn{11}{|c|}{ DILEPTON CUT } \\
\hline & \multirow{2}{*}{$\begin{array}{l}\text { Lepton } \\
\text { ID }\end{array}$} & \multirow{2}{*}{$\begin{array}{c}\text { Cosmic } \\
\text { Ray }\end{array}$} & \multirow{2}{*}{$\begin{array}{l}\text { Opp. } \\
\text { Sign }\end{array}$} & \multirow[b]{2}{*}{ Isolation } & \multirow[b]{2}{*}{$M_{l l}$} & \multirow[b]{2}{*}{$E_{T}$} & \multirow[b]{2}{*}{$\Delta \phi\left(\boldsymbol{E}_{T}, l\right)$} & \multirow[b]{2}{*}{$\Delta \phi\left(\mathbb{E}_{T}, j\right)$} & \multicolumn{3}{|c|}{$N_{j}>10 \mathrm{GeV}$} \\
\hline & & & & & & & & & 0 & 1 & $\geq 2$ \\
\hline TCE-TCE & 2332 & 2332 & 2328 & 2312 & 243 & 7 & 5 & 5 & 2 & 2 & 1 \\
\hline TCE-LCE & 449 & 449 & 439 & 415 & 58 & 1 & 1 & 1 & 1 & 0 & 0 \\
\hline$e-e$ & 2781 & 2781 & 2767 & 2727 & 301 & 8 & 6 & 6 & 3 & 2 & 1 \\
\hline TCM-TCM & 1413 & 1254 & 1250 & 1201 & 135 & 8 & 5 & 4 & 0 & 3 & 1 \\
\hline TCM-CMX & 988 & 986 & 986 & 983 & 108 & 3 & 3 & 1 & 0 & 1 & 0 \\
\hline CMX-CMX & 190 & 185 & 185 & 176 & 18 & 1 & 1 & 1 & 0 & 1 & 0 \\
\hline TCM-CMI & 613 & 604 & 603 & 587 & 68 & 4 & 1 & 1 & 1 & 0 & 0 \\
\hline CMX-CMI & 206 & 202 & 201 & 196 & 24 & 1 & 0 & 0 & 0 & 0 & 0 \\
\hline$\mu-\mu$ & 3410 & 3231 & 3225 & 3143 & 353 & 17 & 10 & 7 & 1 & 5 & 1 \\
\hline TCE-TCM & 38 & 38 & 30 & 27 & 27 & 9 & 8 & 8 & 2 & 3 & 3 \\
\hline TCE-CMX & 9 & 9 & 9 & 6 & 6 & 4 & 4 & 4 & 1 & 1 & 2 \\
\hline TCE-CMI & 11 & 11 & 10 & 10 & 10 & 1 & 1 & 1 & 1 & 0 & 0 \\
\hline TCM-LCE & 5 & 5 & 5 & 4 & 4 & 2 & 2 & 2 & 0 & 1 & 1 \\
\hline CMX-LCE & 0 & 0 & 0 & 0 & 0 & 0 & 0 & 0 & 0 & 0 & 0 \\
\hline$e-\mu$ & 63 & 63 & 54 & 47 & 47 & 16 & 15 & 15 & 4 & 5 & 6 \\
\hline$l l l$ & 2 & 2 & 2 & 2 & 1 & 1 & 1 & 1 & 0 & 0 & 1 \\
\hline Total & 6256 & 6077 & 6048 & 5919 & 702 & 42 & 32 & 29 & 8 & 12 & 9 \\
\hline
\end{tabular}

Table 5.3: Results from the opposite-sign dilepton analysis of Run I data. 


\begin{tabular}{|c|c|c|c|c|c|c|}
\hline \multirow[b]{3}{*}{ Category } & \multicolumn{3}{|c|}{ This Analysis } & \multicolumn{3}{|c|}{ Top Dilepton } \\
\hline & \multicolumn{3}{|c|}{$N_{j}>10 \mathrm{GeV}$} & \multicolumn{3}{|c|}{$N_{j}>10 \mathrm{GeV}$} \\
\hline & 0 & 1 & $\geq 2$ & 0 & 1 & $\geq 2$ \\
\hline TCE-TCE & 2 & 2 & 1 & 2 & 2 & 1 \\
\hline TCE-LCE & 1 & 0 & 0 & 1 & 0 & 0 \\
\hline$e-e$ & 3 & 2 & 1 & 3 & 2 & 1 \\
\hline TCM-TCM & 0 & 3 & 1 & 1 & 2 & 1 \\
\hline TCM-CMX & 0 & 1 & 0 & 0 & 1 & 0 \\
\hline CMX-CMX & 0 & 1 & 0 & 0 & 1 & 0 \\
\hline TCM-CMI & 1 & 0 & 0 & 0 & 0 & 0 \\
\hline CMX-CMI & 0 & 0 & 0 & 0 & 0 & 0 \\
\hline$\mu-\mu$ & 1 & 5 & 1 & 1 & 4 & 1 \\
\hline TCE-TCM & 2 & 3 & 3 & 2 & 3 & $3^{*}$ \\
\hline TCE-CMX & 1 & 1 & 2 & 1 & 1 & 2 \\
\hline TCE-CMI & 1 & 0 & 0 & 1 & 0 & 0 \\
\hline TCM-LCE & 0 & 1 & 1 & 0 & 1 & 1 \\
\hline CMX-LCE & 0 & 0 & 0 & 0 & 0 & 0 \\
\hline$e-\mu$ & 4 & 5 & 6 & 4 & 5 & 6 \\
\hline$l l l$ & 0 & 0 & 1 & 0 & 0 & $1^{*}$ \\
\hline Total & 8 & 12 & 9 & 8 & 11 & 9 \\
\hline
\end{tabular}

Table 5.4: Comparison between this analysis and the original top dilepton analysis for events which have passed all cuts except the 2-jet cut. In the top dilepton note [28], the trilepton event has been assigned to the TCM-TCM category. Here, we have moved it to the separate trilepton category. 
in detail to determine where any discrepancy arose between the two analyses. The explanations below give particular insight into the precision of both analyses and the difficulty of reproducing comparatively recent results.

The TCM-CMI event (Run \#64934/Event \#843315) contains 0 jets and raw $\mathbb{E}_{T}$ of 16.0 GeV. The event passes the $\mathbb{E}_{T}$ cut in this analysis but did not in the top dilepton analysis. The discrepancy occurs due to slight differences in the beam-constrained momenta of the muons, possibly due to changes over time in the definition of the beam spot location. Because the missing energy calculation is corrected for beamconstrained muons, the $\mathbb{E}_{T}$ calculation is boosted just above the threshold of the event filter $\left(\mathbb{E}_{T} \geq 25 \mathrm{GeV}\right)$. A comparison of the kinematic variables measured by the two analyses is shown in Table 5.5.

\begin{tabular}{ccc} 
& Top Dilepton & Current Analysis \\
\hline$p_{T}(\mathrm{TCM})$ & $39.2 \mathrm{GeV} / \mathrm{c}$ & $39.6 \mathrm{GeV} / \mathrm{c}$ \\
$p_{T}(\mathrm{CMI})$ & $70.6 \mathrm{GeV} / \mathrm{c}$ & $72.1 \mathrm{GeV} / \mathrm{c}$ \\
$\mathbb{E}_{T}^{\text {corr }}$ & $24.0 \mathrm{GeV}$ & $25.1 \mathrm{GeV}$
\end{tabular}

Table 5.5: Discrepancies in the beam constrained momentum measured by the top dilepton analysis and our opposite-sign analysis arise due to changes in the beam spot definition. This causes the newly calculated $\mathbb{E}_{T}$ to pass the missing energy cut.

In the TCM-TCM dilepton category, the top analysis found one 0-jet event and two 1-jet events, while we observe zero 0 -jet and three 1 -jet events. The disagreement occurs not because one event has simply moved from one jet bin to the another. Instead, there are two events that each pass one analysis but fail the other.

The first (Run \#69853/Event \#54784) appears in our analysis but fails the top search. In the original top analysis, the standard cosmic ray filter was applied to the data. A second cosmic filter routine [35] was tested on the sample and found exactly the same events that were tagged by the original routine, plus one additional event. While the second cosmic ray algorithm was not formally adopted by the TOPFND 
code, this particular event (Run \#69853/Event \#54784) was removed from the top dilepton sample.

The other TCM-TCM event, (Run \#65408/Event \#84362), passes all cuts in the top dilepton analysis, save for the 2-jet requirement. However, it is removed at an earlier stage in our analysis. In this case, the explanation again appears to be associated with the missing energy calculation. The event contains two muons with $p_{T}=98.6$ and $140.7 \mathrm{GeV}$, and the corrected missing energy is $\mathbb{E}_{T}^{\text {corr }}=49.5 \mathrm{GeV}$. Slight changes to the beam-constrained momenta have caused the missing energy to be reduced below the $50 \mathrm{GeV}$ threshold, at which point the opening angle cuts are applied $\left(25 \leq \mathbb{E}_{T} \leq 50 \mathrm{GeV}\right)$. Indeed, the angle between one of the leptons and the missing energy is $\Delta \phi\left(\mathbb{E}_{T}, l\right)=14^{\circ}$, which causes the event to fail.

\subsection{Like-sign analysis}

Having satisfied ourselves that the opposite-sign dilepton analysis has been accurately reproduced, we now move on to the same-sign analysis of the data. The results are presented in Table 5.6. As expected, extremely few (31) of the events which pass the lepton identification and cosmic ray filter contain like-sign dileptons. Approximately $90 \%$ of these events are removed by the isolation requirement and invariant mass filter. All three remaining events, including the trilepton event which also passes the opposite-sign analysis, pass the $\mathbb{E}_{T}$ and 2-jet requirements.

\begin{tabular}{crc} 
Run & Event & Dilepton Type \\
\hline 57621 & 45230 & $l l l($ TCE-TCM $)$ \\
61074 & 103772 & $e \mu($ TCE-TCM $)$ \\
68592 & 219028 & $e \mu($ TCE-TCM $)$
\end{tabular}

Each of these events has been encountered before in other analyses, and therefore it is not entirely unexpected that they should pass the same-sign dilepton analysis. The third event (Run \#68592/Event \#219028) contains a muon that does not match 


\begin{tabular}{|c|c|c|c|c|c|c|c|c|c|c|c|}
\hline \multirow[b]{3}{*}{ Category } & \multicolumn{11}{|c|}{ DILEPTON CUT } \\
\hline & \multirow{2}{*}{$\begin{array}{c}\text { Lepton } \\
\text { ID }\end{array}$} & \multirow{2}{*}{$\begin{array}{c}\text { Cosmic } \\
\text { Ray }\end{array}$} & \multirow{2}{*}{$\begin{array}{l}\text { Same } \\
\text { Sign }\end{array}$} & \multirow[b]{2}{*}{ Isolation } & \multirow[b]{2}{*}{$M_{l l}$} & \multirow[b]{2}{*}{$E_{T}$} & \multirow[b]{2}{*}{$\Delta \phi\left(\mathbb{E}_{T}, l\right)$} & \multirow[b]{2}{*}{$\Delta \phi\left(\mathbb{E}_{T}, j\right)$} & \multicolumn{3}{|c|}{$N_{j}>10 \mathrm{GeV}$} \\
\hline & & & & & & & & & 0 & 1 & $\geq 2$ \\
\hline TCE-TCE & 2332 & 2332 & 4 & 1 & 0 & 0 & 0 & 0 & & 0 & 0 \\
\hline TCE-LCE & 449 & 449 & 10 & 2 & 0 & 0 & 0 & 0 & 0 & 0 & 0 \\
\hline$e-e$ & 2781 & 2781 & 14 & 3 & 0 & 0 & 0 & 0 & 0 & 0 & 0 \\
\hline TCM-TCM & 1413 & 1254 & 4 & 0 & 0 & 0 & 0 & 0 & 0 & 0 & 0 \\
\hline TCM-CMX & 988 & 986 & 0 & 0 & 0 & 0 & 0 & 0 & & 0 & 0 \\
\hline CMX-CMX & 190 & 185 & 0 & 0 & 0 & 0 & 0 & 0 & 0 & 0 & 0 \\
\hline TCM-CMI & 613 & 604 & 1 & 1 & 0 & 0 & 0 & 0 & 0 & 0 & 0 \\
\hline CMX-CMI & 206 & 202 & 1 & 1 & 0 & 0 & 0 & 0 & 0 & 0 & 0 \\
\hline$\mu-\mu$ & 3410 & 3231 & 6 & 2 & 0 & 0 & 0 & 0 & 0 & 0 & 0 \\
\hline TCE-TCM & 38 & 38 & 8 & 2 & 2 & 2 & 2 & 2 & 0 & 0 & 2 \\
\hline TCE-CMX & 9 & 9 & 0 & 0 & 0 & 0 & 0 & 0 & 0 & 0 & 0 \\
\hline TCE-CMI & 11 & 11 & 1 & 0 & 0 & 0 & 0 & 0 & 0 & 0 & 0 \\
\hline TCM-LCE & 5 & 5 & 0 & 0 & 0 & 0 & 0 & 0 & 0 & 0 & 0 \\
\hline CMX-LCE & 0 & 0 & 0 & 0 & 0 & 0 & 0 & 0 & 0 & 0 & 0 \\
\hline$e-\mu$ & 63 & 63 & 9 & 2 & 2 & 2 & 2 & 2 & 0 & 0 & 2 \\
\hline$l l l$ & 2 & 2 & 2 & 2 & 1 & 1 & 1 & 1 & 0 & 0 & 1 \\
\hline Total & 6256 & 6077 & 31 & 9 & 3 & 3 & 3 & 3 & 0 & 0 & 3 \\
\hline
\end{tabular}

Table 5.6: Results from the like-sign dilepton analysis of Run I data. 


\begin{tabular}{|c|c|c|c|}
\hline \multirow[b]{2}{*}{ Kinematic variable } & \multicolumn{3}{|c|}{ Run \# / Event \# } \\
\hline & $61074 / 103772$ & $68592 / 219028$ & $57621 / 45230$ \\
\hline Dilepton category & $e^{-} \mu^{-}(\mathrm{TCE}-\mathrm{TCM})$ & $e^{+} \mu^{+}(\mathrm{TCE}-\mathrm{TCM})$ & $e^{+} \mu^{+} \mu^{-}$(TCE-TCM) \\
\hline$E_{T}(e)$ & $27.9 \mathrm{GeV}$ & $57.5 \mathrm{GeV}$ & $49.0 \mathrm{GeV}$ \\
\hline$\eta(e)$ & -0.42 & -0.29 & 0.77 \\
\hline$\phi(e)$ & $120^{\circ}$ & $84^{\circ}$ & $340^{\circ}$ \\
\hline$E_{T}(\mu)$ & $25.1 \mathrm{GeV}$ & $89.1 \mathrm{GeV}$ & $25.4 \mathrm{GeV}$ \\
\hline$\eta(\mu)$ & 0.06 & -0.49 & -0.48 \\
\hline$\phi(\mu)$ & $318^{\circ}$ & $165^{\circ}$ & $115^{\circ}$ \\
\hline$E\left(j_{1}\right)$ & $115.4 \mathrm{GeV}$ & $55.9 \mathrm{GeV}$ & $36.5 \mathrm{GeV}$ \\
\hline$E_{T}\left(j_{1}\right)$ & $104.3 \mathrm{GeV}$ & $53.5 \mathrm{GeV}$ & $32.2 \mathrm{GeV}$ \\
\hline$\eta\left(j_{1}\right)$ & 0.46 & -0.30 & 0.51 \\
\hline$\phi\left(j_{1}\right)$ & $226^{\circ}$ & $264^{\circ}$ & $254^{\circ}$ \\
\hline$E\left(j_{2}\right)$ & $52.0 \mathrm{GeV}$ & $37.2 \mathrm{GeV}$ & $32.4 \mathrm{GeV}$ \\
\hline$E_{T}\left(j_{2}\right)$ & $52.0 \mathrm{GeV}$ & $29.2 \mathrm{GeV}$ & $30.4 \mathrm{GeV}$ \\
\hline$\eta\left(j_{2}\right)$ & -0.04 & 0.73 & -0.37 \\
\hline$\phi\left(j_{2}\right)$ & $92^{\circ}$ & $266^{\circ}$ & $190^{\circ}$ \\
\hline$E\left(j_{3}\right)$ & $37.2 \mathrm{GeV}$ & - & - \\
\hline$E_{T}\left(j_{3}\right)$ & $37.2 \mathrm{GeV}$ & - & - \\
\hline$\eta\left(j_{3}\right)$ & 0.04 & - & - \\
\hline$\phi\left(j_{3}\right)$ & $309^{\circ}$ & - & - \\
\hline$E_{T}$ & $60.6 \mathrm{GeV}$ & $85.3 \mathrm{GeV}$ & $51.4 \mathrm{GeV}$ \\
\hline$\phi\left(\mathbb{E}_{T}\right)$ & $34^{\circ}$ & $1^{\circ}$ & $83^{\circ}$ \\
\hline$z$-vertex & $-3.5 \mathrm{~cm}$ & $19.6 \mathrm{~cm}$ & $-10.8 \mathrm{~cm}$ \\
\hline
\end{tabular}

Table 5.7: Kinematic properties of the two same-sign dilepton events and one trilepton event. For event 57621/45230, the second set of lepton variables is for the $\mu^{-}$. The second muon $\left(\mu^{+}\right)$has $p_{T}=21 \mathrm{GeV}$ and is located within the primary jet $\left(j_{1}\right)$. 
well with its associated $z$-vertex $(|\Delta z|=2.5 \mathrm{~cm})$ or with the beam position (impact parameter $d_{0}=0.25 \mathrm{~cm}$ ). The momentum before beam-constraining is $846 \mathrm{GeV}$, and the charge afterwards cannot not be determined. The most likely explanation is that the muon track has been badly mismeasured and that this should be an opposite-sign event.

The other $e \mu$ event (Run \#61074/Event \#103772, shown in Figure 5.1) has been thoroughly studied in the lepton + jets analysis. (Recall that this particular top signature is produced when one $W$ decays leptonically while the other decays hadronically.) The muon $\left(p_{T}=25.1 \mathrm{GeV}\right)$ is located inside one of the jets and is highly non-isolated. This jet, along with another of the three jets, is also $b$-tagged by the SECVTX routine. In other words, this appears to be a case where one of the $b$ quarks has decayed semileptonically, and the muon is surrounded by the hadronic activity of the parent $b$ jet. This Standard Model $t \bar{t}$ process is a background to the same-sign top dilepton channel, although the expected contribution is on the order of $1 / 4$ of an event. This type of event will be addressed in greater detail in Section 8.5.

Finally, the trilepton event (Run \#57621/Event \#45230, shown in Figure 5.2) passes both the opposite- and same-sign analyses. The event contains an oppositesign $e^{+} \mu^{-}$pair, and is $b$-tagged by the "soft lepton tag" algorithm. The SLT routine searches for soft leptons (as opposed to stiffer momentum leptons from $W^{ \pm}$) within jets as evidence of the $b$ quark decay $b \rightarrow c l \nu_{l}$ or $b \rightarrow c \rightarrow s l \nu_{l}$, where $l$ is either an electron or muon. In the trilepton event, the $p_{T}=21 \mathrm{GeV}$ muon used by the SLT to tag the jet also happens to pass all of the kinematic requirements for muon ID. Therefore, it is considered as a third high- $p_{T}$ lepton in the event.

The probability of a $b$ jet creating a high- $p_{T}(>20 \mathrm{GeV})$ lepton which passes the lepton ID requirements is extremely small. The percentage of $t \bar{t}$ dilepton candidate events which contain such a third lepton is calculated to be approximately $(0.3 \pm 0.2) \%$. In the top dilepton analysis, 9 opposite-sign events are observed, on top of an expected background of $2.1 \pm 0.4$ events. If one assumes the presence of 7 events from signal $\left(N_{\text {obs }}-N_{\text {bkgd }}(\exp )\right)$, then the $0.3 \%$ probability implies that on the order of 0.02 trilepton events would be expected for a sample size equivalent to the Run I integrated luminosity. 

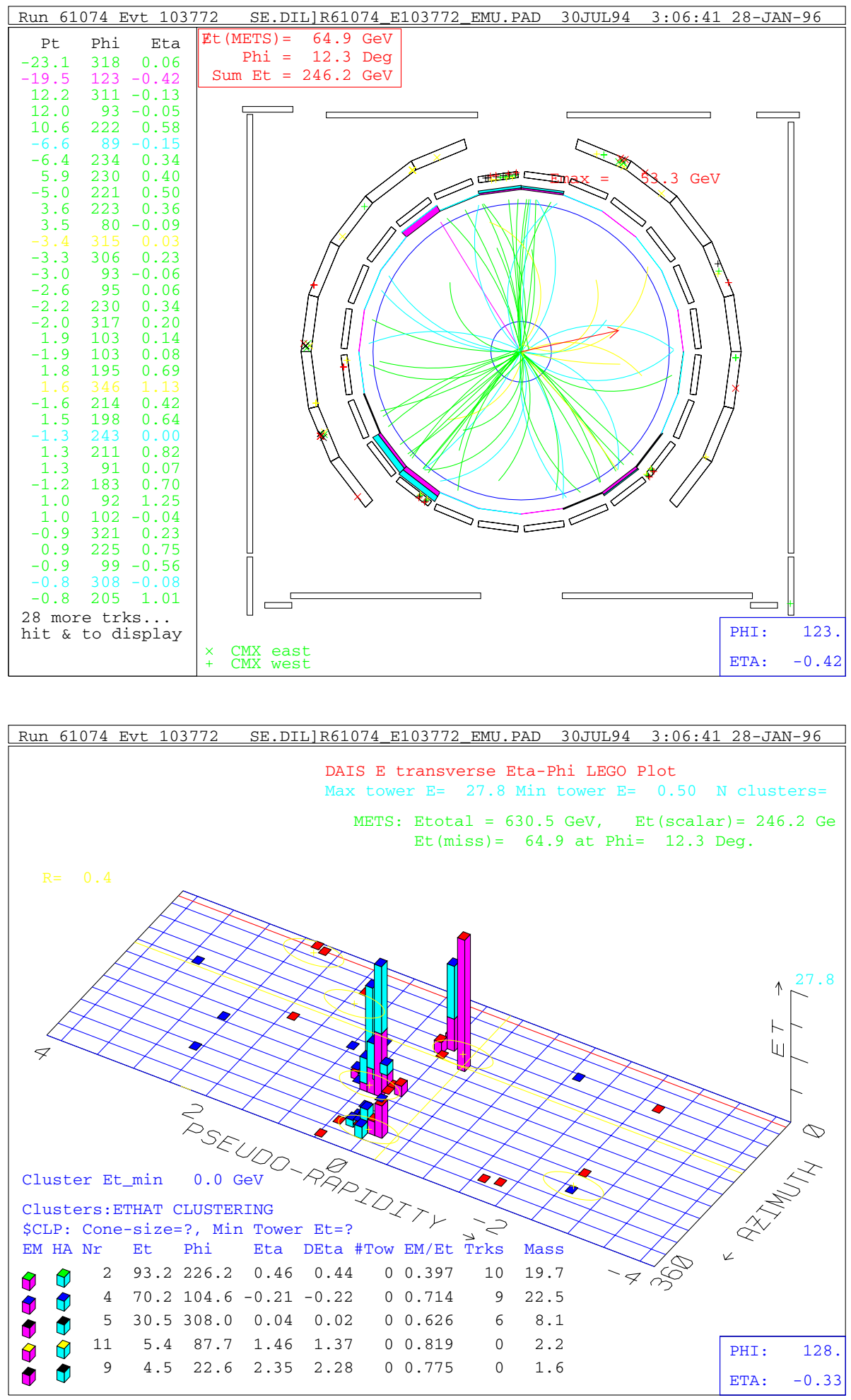

Figure 5.1: Event display for the same-sign dilepton event 61074/103772. The top diagram presents a transverse view of the tracks in the CTC. The red arrow indicates the direction of the $\mathbb{E}_{T}$. The lego display in the lower diagram illustrates the distribution of EM (purple) and hadronic (cyan) calorimeter energy. 

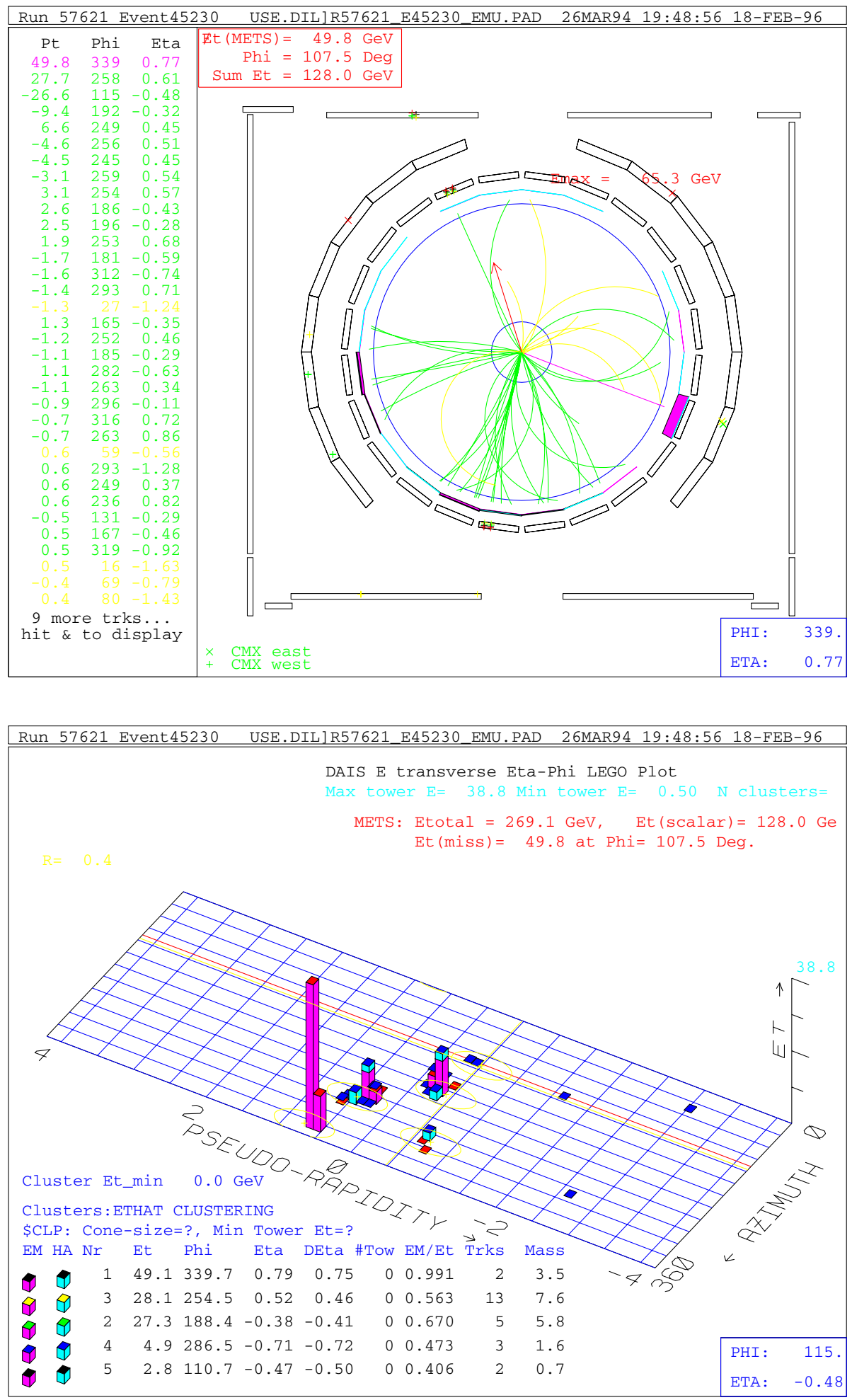

Figure 5.2: Event display for the same-sign dilepton event 57621/45230. The top diagram presents a transverse view of the tracks in the CTC. The red arrow indicates the direction of the $\mathbb{E}_{T}$. The lego display in the lower diagram illustrates the distribution of EM (purple) and hadronic (cyan) calorimeter energy. 


\subsection{Summary}

Regardless of the true nature of these three events, they constitute the observed signal for the like-sign dilepton analysis in Run I. One same-sign dilepton event may appear to be an opposite-sign event where the muon charge has been mismeasured, and the other two events could possibly be due to semileptonic $b$ decay. Determination of the exact mechanisms behind these events is a subject for future analysis, especially if one wishes to further optimize the event selection criteria to reduce the background acceptance. For the present, we accept this as our observed signal and proceed to calculate the expected contributions to the same-sign dilepton channel from gluino pair production and background processes. 


\section{Chapter 6}

\section{Monte Carlo Acceptance}

As shown in the previous chapter, we have observed 3 events in the data (2 likesign $e-\mu$ events and 1 trilepton event) which pass the same-sign analysis. This alone is not sufficient evidence for the presence of gluino events in the Run I data sample. In order to make any kind of definitive statement, we must estimate the number of events expected from the signal process, $\tilde{g} \rightarrow t \tilde{t}$, and from various background sources. The contribution from background will be addressed later in Chapter 8 .

Several steps are involved in determining the expected number of events from signal:

- First, we must calculate the cross section for gluino pair production in $p \bar{p}$ collisions at the Tevatron energy $(\sqrt{s}=1.8 \mathrm{TeV})$. Because the gluino and squark masses are unknown, the cross section is calculated for a range of gluino and squark masses.

- Next, a Monte Carlo generator is employed to simulate the physics of gluino production and decay. This produces virtual events which are fully evolved from the initially produced particles down through all decay products. Only the physics of particle production and decay are simulated, and the result is detector independent.

- The events generated by the Monte Carlo are then passed through a simulation of the CDF detector. The trajectory is calculated for each virtual particle as 
it interacts with the various detector subsystems and inactive concentrations of mass (such as the toroid). The estimated response of the detector is mapped out, creating fully simulated events which are comparable to actual data.

- The simulated data is processed through the same analysis routines used for the data. From this, we calculate the acceptance rate (i.e., the percentage of virtual gluino events which pass the analysis) for a range of accessible gluino and squark masses.

Further discussion of each of these steps follows in the sections below. For each gluino-squark combination, the acceptance may be combined with the $\tilde{g} \tilde{g}$ cross section and Run I luminosity to arrive at an estimate for the number of like-sign dilepton events we should expect from signal in Run I. Any conclusions based on the acceptance are reserved for Chapter 9, once the systematic uncertainty on the acceptance and the contribution from background have been determined.

\subsection{Minimal Supersymmetric Standard Model}

The finer details of constructing a general supersymmetric theory are beyond the scope of this document and are better left to other sources [3, 37, 38]. As we stressed earlier, this is a signature-based search and is independent of any particular flavor of Supersymmetry. However, we briefly return to a discussion of SUSY to further motivate the direction taken in modeling the signal in Monte Carlo.

In the Standard Model, a spin-1/2 fermion is represented by a two-component (Weyl) spinor to describe the two spin states. In the Dirac (four-component) representation, the fermion consists of two two-component Weyl fermions (left- and righthanded). Meanwhile, bosons are complex scalar fields and therefore have two degrees of freedom. After supersymmetric partners are assigned to all of the Standard Model particles, each particle and its superpartner are paired to form supermultiplets, along with their associated superfields. The two types of superfields are:

- chiral superfields, consisting of one two-component Majorana fermion field, $\psi$, and a complex scalar field, $S$, termed a sfermion; and 


\begin{tabular}{|c|c|c|c|c|}
\hline \multicolumn{2}{|l|}{ Names } & spin 0 & $\operatorname{spin} 1 / 2$ & $\left(S U(3)_{C}, S U(2)_{L}, U(1)_{Y}\right)$ \\
\hline $\begin{array}{l}\text { squarks, quarks } \\
\text { (×3 families })\end{array}$ & $\begin{array}{l}Q \\
\bar{u} \\
\bar{d}\end{array}$ & $\begin{array}{c}\left(\begin{array}{cc}\tilde{u}_{L} & \tilde{d}_{L}\end{array}\right) \\
\tilde{u}_{R}^{*} \\
\tilde{d}_{R}^{*}\end{array}$ & $\begin{array}{c}\left(\begin{array}{ll}u_{L} & d_{L}\end{array}\right) \\
\tilde{u}_{R}^{\dagger} \\
\tilde{d}_{R}^{\dagger}\end{array}$ & $\begin{array}{c}\left(\mathbf{3}, \mathbf{2}, \frac{1}{6}\right) \\
\left(\overline{\mathbf{3}}, \mathbf{1},-\frac{2}{3}\right) \\
\left(\overline{\mathbf{3}}, \mathbf{1}, \frac{1}{3}\right)\end{array}$ \\
\hline $\begin{array}{l}\text { sleptons, leptons } \\
(\times 3 \text { families })\end{array}$ & $\begin{array}{l}L \\
\bar{e} \\
\end{array}$ & $\begin{array}{c}\left(\begin{array}{ll}\tilde{\nu} & \tilde{e}_{L}\end{array}\right) \\
\tilde{e}_{R}^{*} \\
\end{array}$ & $\begin{array}{c}\left(\begin{array}{cc}\nu & e_{L}\end{array}\right) \\
e_{R}^{\dagger}\end{array}$ & $\begin{array}{c}\left(\mathbf{1}, \mathbf{2},-\frac{1}{2}\right) \\
(\overline{\mathbf{1}}, \mathbf{1}, 1) \\
\end{array}$ \\
\hline Higgs, higginos & $\begin{array}{l}H_{u} \\
H_{d}\end{array}$ & $\begin{array}{l}\left(\begin{array}{ll}H_{u}^{+} & H_{u}^{0}\end{array}\right) \\
\left(\begin{array}{ll}H_{d}^{0} & H_{d}^{-}\end{array}\right)\end{array}$ & $\begin{array}{l}\left(\begin{array}{ll}\tilde{H}_{u}^{+} & \tilde{H}_{u}^{0}\end{array}\right) \\
\left(\begin{array}{ll}\tilde{H}_{d}^{0} & \tilde{H}_{d}^{-}\end{array}\right)\end{array}$ & $\begin{array}{l}\left(\mathbf{1}, \mathbf{2},+\frac{1}{2}\right) \\
\left(\mathbf{1}, \mathbf{2},-\frac{1}{2}\right)\end{array}$ \\
\hline
\end{tabular}

Table 6.1: Chiral supermultiplets in the Minimal Supersymmetric Standard Model.

\begin{tabular}{|c|c|c|c|}
\hline Names & spin $1 / 2$ & spin 1 & $\left(S U(3)_{C}, S U(2)_{L}, U(1)_{Y}\right)$ \\
\hline \hline gluino, gluon & $\tilde{g}$ & $g$ & $(\mathbf{8}, \mathbf{1}, 0)$ \\
\hline bino, B boson & $\tilde{B}^{0}$ & $B^{0}$ & $(\mathbf{1}, \mathbf{1}, 0)$ \\
\hline winos, W bosons & $\left(\tilde{W}^{ \pm} \tilde{W}^{0}\right)$ & $\left(W^{ \pm} W^{0}\right)$ & $(\mathbf{1}, \mathbf{3}, 0)$ \\
\hline \hline
\end{tabular}

Table 6.2: Gauge supermultiplets in the Minimal Supersymmetric Standard Model.

- gauge superfields, consisting of a massless gauge field, $F_{\mu \nu}$, and a two-component Majorana fermion field, $\lambda$, known as a gaugino.

Each Dirac fermion actually requires two chiral supermultiplets. As an example, the electron is contained in the supermultiplets $\left(e_{L}, \tilde{e}_{L}\right)$ and $\left(e_{R}, \tilde{e}_{R}\right)$. Although labelled as left- or right-handed, sfermions are scalar particles, and the chirality refers to the Standard Model fermion, not its superpartner. All of the chiral and gauge supermultiplets are shown in Tables 6.1 and 6.2.

One of the more popular frameworks for Supersymmetry is the Minimal Supersymmetric Standard Model (MSSM). The MSSM is "minimal" in the sense that the superpotential describing all of the allowed interactions contains a sufficient number 
of terms to produce a phenomenologically viable model. The interactions are expressed in terms of the chiral superfields $\bar{u}, \bar{d}, \bar{e}, Q, H_{u}$, and $H_{d}$ corresponding to the supermultiplets given in Table 6.1. The $y_{u}, y_{d}$, and $y_{e}$ are the dimensionless Yukawa couplings (in fact, $3 \times 3$ matrices when including all families). All $S U(3)_{C}$ color, $S U(2)_{L}$ weak isospin, and family indices have been suppressed for simplicity. The superpotential of the MSSM is given by:

$$
W_{M S S M}=\bar{u} \mathbf{y}_{\mathbf{u}} Q H_{u}-\bar{d} \mathbf{y}_{\mathbf{d}} Q H_{d}-\bar{e} \mathbf{y}_{\mathbf{e}} L H_{d}+\mu H_{u} H_{d}
$$

Of course, nothing prevents one from constructing a more general parameterization of Supersymmetry by including other interaction terms. Among the possibilities are terms such as:

$$
\begin{aligned}
& W_{\Delta L=1}=\frac{1}{2} \lambda^{i j k} L_{i} L_{j} \bar{e}_{k}+\lambda^{\prime i j k} L_{i} Q_{j} \bar{d}_{k}+\mu^{i} L_{i} H_{u} \text { and } \\
& W_{\Delta B=1}=\frac{1}{2} \lambda^{\prime \prime i j k} \bar{u}_{i} \bar{d}_{j} \bar{d}_{k} .
\end{aligned}
$$

However, these interactions are not included in the MSSM because they violate conservation of baryon number (B) or total lepton number (L): $\Delta \mathrm{B}=1$ or $\Delta \mathrm{L}=1$. In the Standard Model, these quantities are believed to be separately conserved, and at present all searches have found no evidence for interactions which violate B or L.

In nature, the reaction $\nu+n \rightarrow p^{+}+e^{-}$is readily observed, while the reaction $\bar{\nu}+n \rightarrow p^{+}+e^{-}$is conspicuously absent. The neutrino and antineutrino are therefore distinct particles, and this leads to the idea of conservation of total lepton number. Lepton number $\mathrm{L}=+1$ is assigned to the electron and neutrino, while $\mathrm{L}=-1$ is assigned to the positron and antineutrino. Furthermore, the individual lepton numbers for each family (electron, muon, tau) appear to be separately conserved. Searches for the decay $\mu \rightarrow e \gamma$ have ruled out the branching ratio for this neutrinoless muon decay mode to $\Gamma\left(\mu^{-} \rightarrow e^{-} \gamma\right) / \Gamma_{\text {total }}<1.2 \times 10^{-11}$, at $90 \%$ confidence level [39]. Instead, the muon preferentially decays via $\mu^{-} \rightarrow e^{-} \overline{\nu_{e}} \nu_{\mu}$. The neutrinos are required only if electron and muon lepton number must be conserved.

Conservation of baryon number was proposed by Stuckelberg in 1938 to explain the stability of the proton. Otherwise, the reaction $p^{+} \rightarrow e^{+} \gamma$ would be allowed by then understood conservation laws. (Of course, this reaction also violates lepton number 


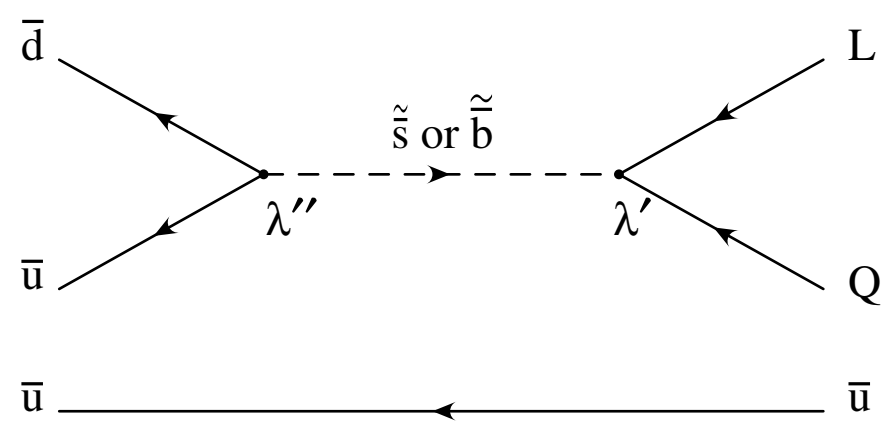

Figure 6.1: In the absence of $R$-parity conservation, proton decay is mediated via an intermediate squark.

conservation, but that may be remedied.) Baryons are assigned baryon number B $=+1$, antibaryons $\mathrm{B}=-1$, and mesons and leptons $\mathrm{B}=0$.

This idea has been extended to that of hadronic flavor and has been given stronger theoretical footing. In the strong and electromagnetic interactions, conversion of a quark from one flavor $(d, u, s, c, b, t)$ to another is forbidden. In the weak interaction, flavor changing is allowed (such as $t \rightarrow b W^{+}$) and is governed by Cabibbo-KobayashiMaskawa mixing between the mass and weak eigenstates. B and L conservation are no longer simply assumed but are a direct consequence of the fact that there exist no renormalizable lagrangian terms which violate B or L.

In Supersymmetry, the inclusion of lepton or baryon number violating terms has potentially disastrous consequences, the most obvious example being the stability of the proton. In the case where any of the $\lambda$ or $\lambda^{\prime}$ coefficients in Eq. 6.2 are non-zero and instead are of order unity, the proton is allowed to decay via an intermediate squark, as shown in Figure 6.1. A variety of decay modes, including $p \rightarrow e^{+} \pi^{0}$, $e^{+} K^{0}, \mu^{+} \pi^{0}$, or $\mu^{+} K^{0}$, become accessible to the proton. The resulting proton lifetime would be measured in terms of hours or minutes, while current estimates place the timescale many orders greater than the lifetime of the universe. The apparent stability of ordinary matter suggests that violation of baryon number is heavily suppressed, if not completely disallowed. Searches for proton decay at facilities such as 
SuperKamiokande in Japan have thus far yielded negative results. Present experimental limits place the proton lifetime at greater than $10^{25}$ years, and the mean decay time into the decay modes listed above is on the order of $10^{32}$ years.

Many other significant constraints on lepton and baryon number violation exist. Rather than impose B and L as fundamental symmetries in supersymmetry, especially since they are known to be violated in electroweak interactions, a new symmetry is proposed which has the effect of eliminating the offending terms in Eq. 6.2. This symmetry is known as " $R$-parity" [40], and the corresponding (multiplicatively conserved) quantum number is defined as

$$
R=(-1)^{3(\mathrm{~B}-\mathrm{L})+2 s}
$$

where $s$ is the spin of the particle. A quick check reveals that all Standard Model particles (including the Higgs boson) have even $R$-parity $(R=+1)$, while all supersymmetric particles (or sparticles) have odd $R$-parity $(R=-1)$. Subsequently, the restriction imposed upon the lagrangian is that only terms where the multiplicative product of $R$ for all fields equals +1 are allowed. All of the interactions in Eq. 6.2 are therefore forbidden by $R$-parity, while those of Eq. 6.1 are allowed.

Adoption of $R$-parity conservation has several implications for phenomenology:

- Interactions and decays involving solely Standard Model particles in the initial state lead to final states including only an even number of sparticles. Thus, supersymmetric particles must be pair produced in proton-antiproton collisions at the Tevatron.

- Sparticle decay may involve only an odd number of supersymmetric products.

- The lightest (lowest mass state) sparticle is, by necessity, absolutely stable as there are no lighter sparticle states into which it may decay and it cannot decay into lighter Standard Model particles. This sparticle is referred to as the "lightest supersymmetric particle," or LSP. A stable, charged LSP (i.e., a chargino, squark, or slepton) would easily be observed through the presence of long-lived tracks in the detector volume. This option is rarely considered 
since such objects would likely have already been discovered. A neutral LSP (neutralino or sneutrino), on the other hand, would interact extremely weakly with ordinary matter and would appear as missing transverse energy, similar to a neutrino. The neutralino is also an attractive candidate for non-baryonic dark matter, which has been proposed to account for $90 \%$ of the missing matter in the universe [41].

\subsection{Cross section for $\tilde{g} \tilde{g}$ production}

The strength of the interaction between SUSY and Standard Model particles is governed by the gauge and Yukawa couplings $g_{s}$ and $\hat{g}_{s}$. Supersymmetry requires that the two couplings be equal. Therefore, gluinos and squarks couple strongly to quarks and gluons and are expected to be produced copiously at the Tevatron.

This search for gluino production operates under the assumption that $R$-parity is a valid symmetry. Therefore, creation of gluinos and squarks at the Tevatron proceeds through pair production via the following reactions $[42,43]$ :

$$
\begin{array}{ll}
\tilde{g} \tilde{g} \text { production }: & q_{i}+\bar{q}_{j}, g+g \rightarrow \tilde{g}_{k}+\tilde{g}_{l} \\
\tilde{q} \overline{\tilde{q}} \text { production }: & q_{i}+\bar{q}_{j}, g+g \rightarrow \widetilde{q}_{k}+\overline{\tilde{q}}_{l} \\
\tilde{q} \tilde{q} \text { production }: & q_{i}+q_{j} \rightarrow \widetilde{q}_{k}+\widetilde{q}_{l} \\
\tilde{q} \tilde{g} \text { production }: & q_{i}+g_{j} \rightarrow \tilde{q}_{k}+\tilde{g}_{l}
\end{array}
$$

Figure 6.2 provides a few examples of Feynman diagrams which yield gluino pair production.

One useful tool for studying gluino and squark production at hadron colliders is PROSPINO [44]. This package was designed for calculating leading order (LO) and next-to-leading order (NLO) cross sections for $\tilde{g} \tilde{g}, \tilde{q} \overline{\tilde{q}}, \tilde{q} \tilde{g}, \tilde{q} \tilde{q}$, and $\tilde{t} \tilde{t}$ in $p p$ or $p \bar{p}$ interactions. The cross sections for these production modes are relatively insensitive to most parameters in Supersymmetry $\left(\tan \beta\right.$, $\mu$, etc.). For $\tilde{g} \tilde{g}$ production, $\sigma_{\tilde{g} \tilde{g}}$ is most strongly affected by the gluino mass, the degenerate (5-flavor) squark mass, and the choice of QCD renormalization scale $\left(Q^{2}\right.$ scale).

While the leading order cross section for gluino pair production is most strongly 
a)

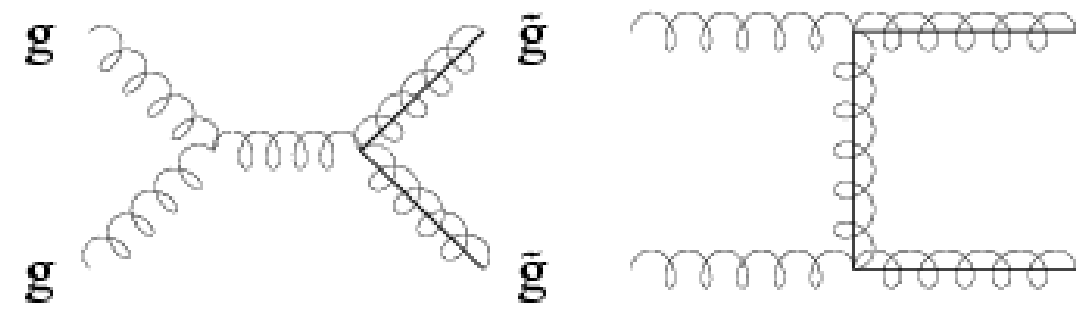

b)
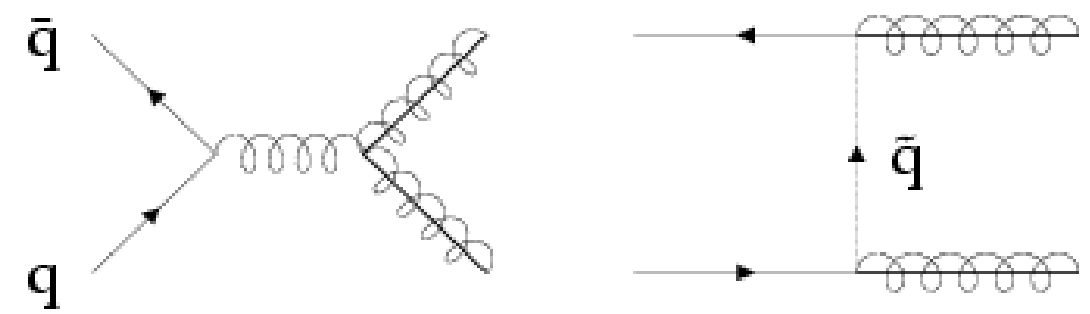

c)
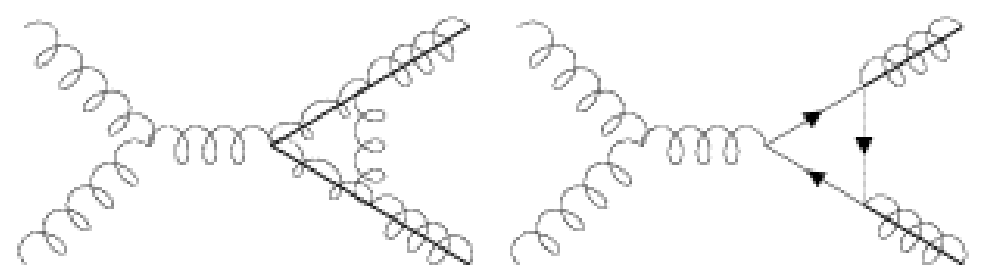

d)
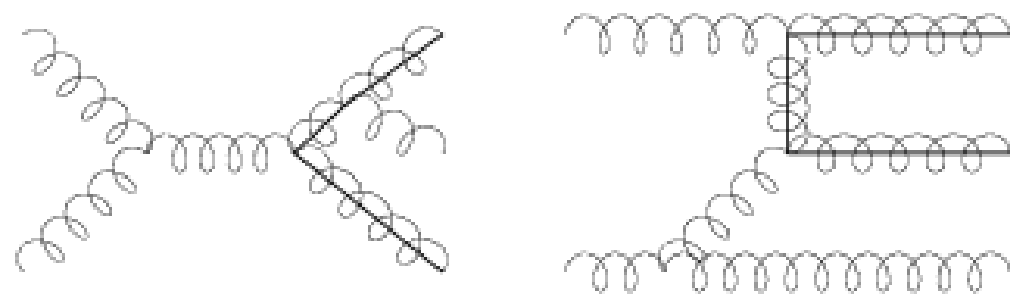

Figure 6.2: Gluino pair production mechanisms: a) Born-level gg diagrams; b) Bornlevel $q \bar{q}$ diagrams; c) Vertex corrections from QCD and SUSY; d) Bremsstrahlung diagrams. Taken from Ref. [42]. 
influenced by the $Q^{2}$ scale, calculation of the NLO contribution tends to reduce the dependency somewhat and boosts the cross section by nearly a factor of two in certain cases. Figure 6.3(a) illustrates this effect in terms of $Q / m_{\tilde{g}}$ for one particular choice of $m_{\tilde{g}}$ and $m_{\tilde{q}}$. Figure $6.3(\mathrm{~b})$ shows the ratio of $\sigma_{N L O}$ to $\sigma_{L O}$ (otherwise known as the $K$ factor) and the variation due to $Q / m_{\tilde{g}}$ for gluino masses up to $500 \mathrm{GeV}$. The upper, middle, and lower curves correspond to choices of $Q / m_{\tilde{g}}$ equal to 2,1 , and $1 / 2$, respectively. These are the standard values we have used when determining the effect of varying the $Q^{2}$ scale. Within the scope of this analysis $\left(200 \leq m_{\tilde{g}} \leq 320\right.$ $\left.\mathrm{GeV} ; m_{\tilde{q}}=1 \mathrm{TeV}\right)$, the $K$ factor ranges from 1.67 to 1.89 .

We have calculated the NLO cross section for $\tilde{g} \tilde{g}$ production using CTEQ 4M and the default QCD scale $\left(s c a f a c=Q / m_{\tilde{g}}=1.0\right.$ in PROSPINO). For each gluino mass between 200 and $320 \mathrm{GeV}$, we have chosen a wide array of squark masses: $200 \leq m_{\tilde{q}} \leq 1000 \mathrm{GeV}$. Figure 6.4 demonstrates how $\sigma_{N L O}$ increases in tandem with $m_{\tilde{q}}$ yet falls quickly with gluino mass, roughly by a factor of two for every $20 \mathrm{GeV}$ increase in $m_{\tilde{g}}$.

The most favorable $\tilde{g} \tilde{g}$ cross section corresponds to the highest choice of $m_{\tilde{q}}$. Supersymmetric theories tend to favor sparticle masses below a TeV. Therefore, squark masses greater than $1 \mathrm{TeV}$ were not pursued. In Figure 6.5 we have plotted the NLO cross section as a function of $m_{\tilde{g}}$ for the choice of $m_{\tilde{q}}=1 \mathrm{TeV}$. In order to demonstrate the dependency of $\sigma_{N L O}$ upon the QCD scale, error curves are shown where $Q^{2}$ has been varied by a factor of 4 (scafac $=Q / m_{\tilde{q}}=0.5$ and 2.0 in PROSPINO). Due to the rapid fall-off in the $\tilde{g} \tilde{g}$ cross-section, gluino masses above $320 \mathrm{GeV}$ are not considered in this analysis.

\subsection{Monte Carlo samples}

There are two constraints which limit the range of gluino masses we can explore in this analysis. First, there is the limiting case of $m_{\tilde{g}}>m_{t}$. The gluino mass must be sufficiently larger than top in order to provide room for lighter supersymmetric particles for the gluino to decay into. In the region where $m_{\tilde{g}}-m_{t}<10-15 \mathrm{GeV}$, understanding the phenomenology becomes quite complicated. However, as we have 

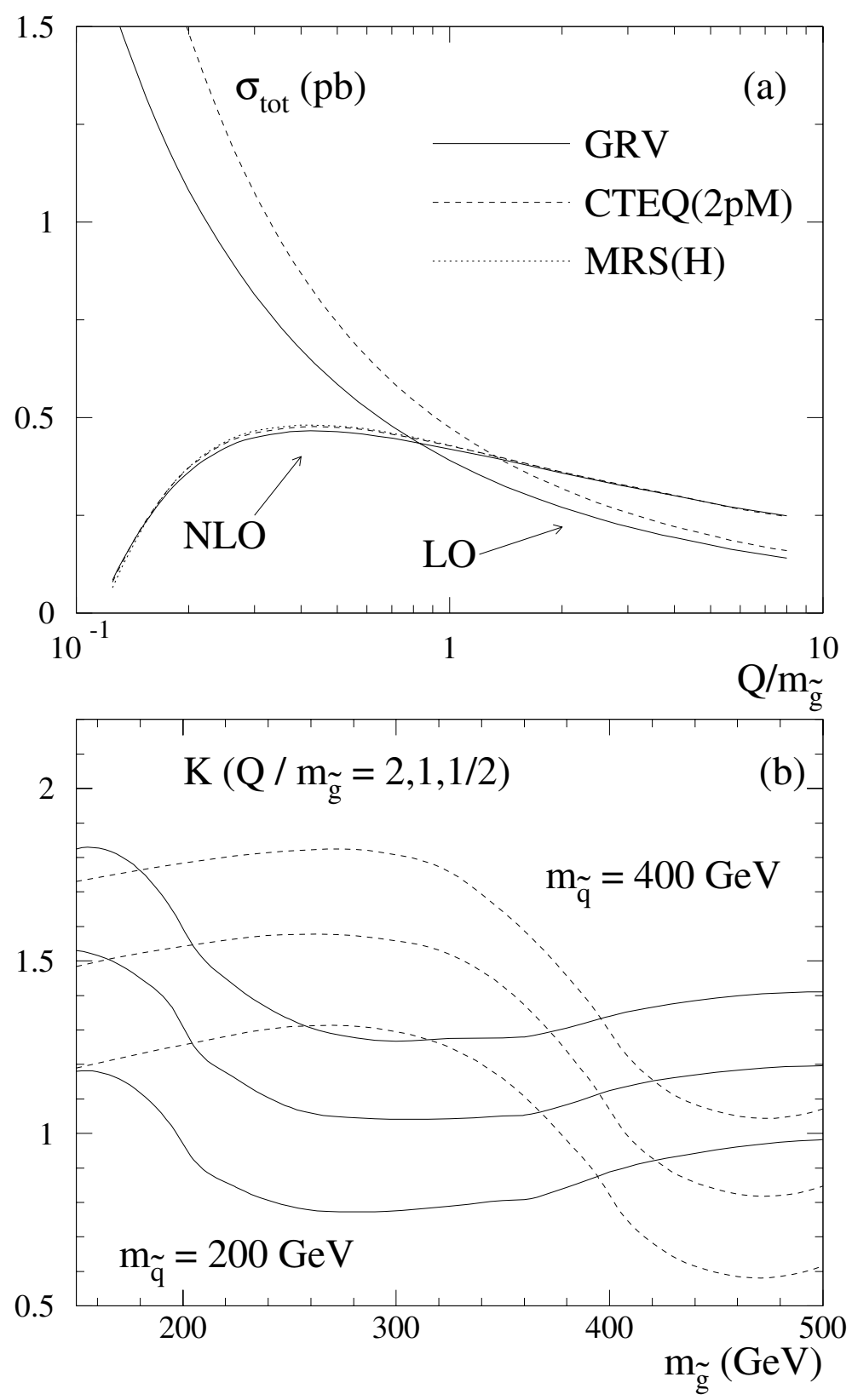

Figure 6.3: (a) Dependence of the total cross section for $\tilde{g} \tilde{g}$ production at the Tevatron $(\sqrt{s}=1.8 \mathrm{TeV})$ upon the choice of $Q^{2}$ scale. The sensitivity to $Q / m_{\tilde{g}}$ is shown for different choices of PDF and mass parameters $m_{\tilde{g}}=250 \mathrm{GeV}$ and $m_{\tilde{q}}=200 \mathrm{GeV}$. (b) $K=\sigma_{N L O} / \sigma_{L O}$ is shown for $Q / m_{\tilde{g}}=2,1,1 / 2$ (corresponding to the upper, middle, and lower curves) using the GRV parton density function. Taken from Ref. [42]. 
Cross section and number of events in $106.1 \mathrm{pb}^{-1}$

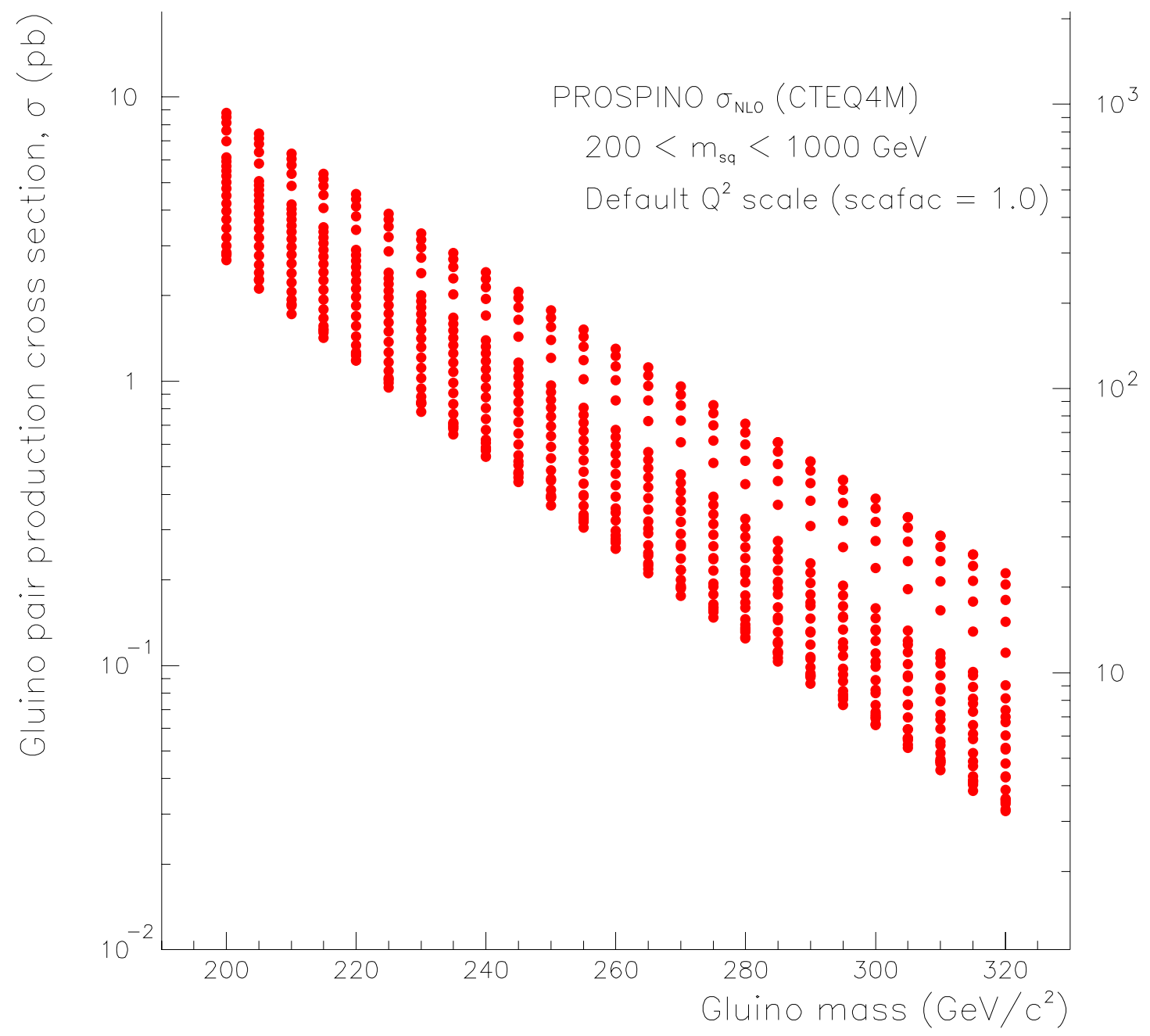

Figure 6.4: Calculation of $\tilde{g} \tilde{g}$ cross section (in pb) from PROSPINO. Values were calculated using the CTEQ $4 \mathrm{M}$ PDF and default $Q^{2}$ scale for a range of squark masses from 200 to $1000 \mathrm{GeV}$. The scale at right represents the total number of expected $\tilde{g} \tilde{g}$ events in Run I, regardless of decay mode or detection efficiency. 


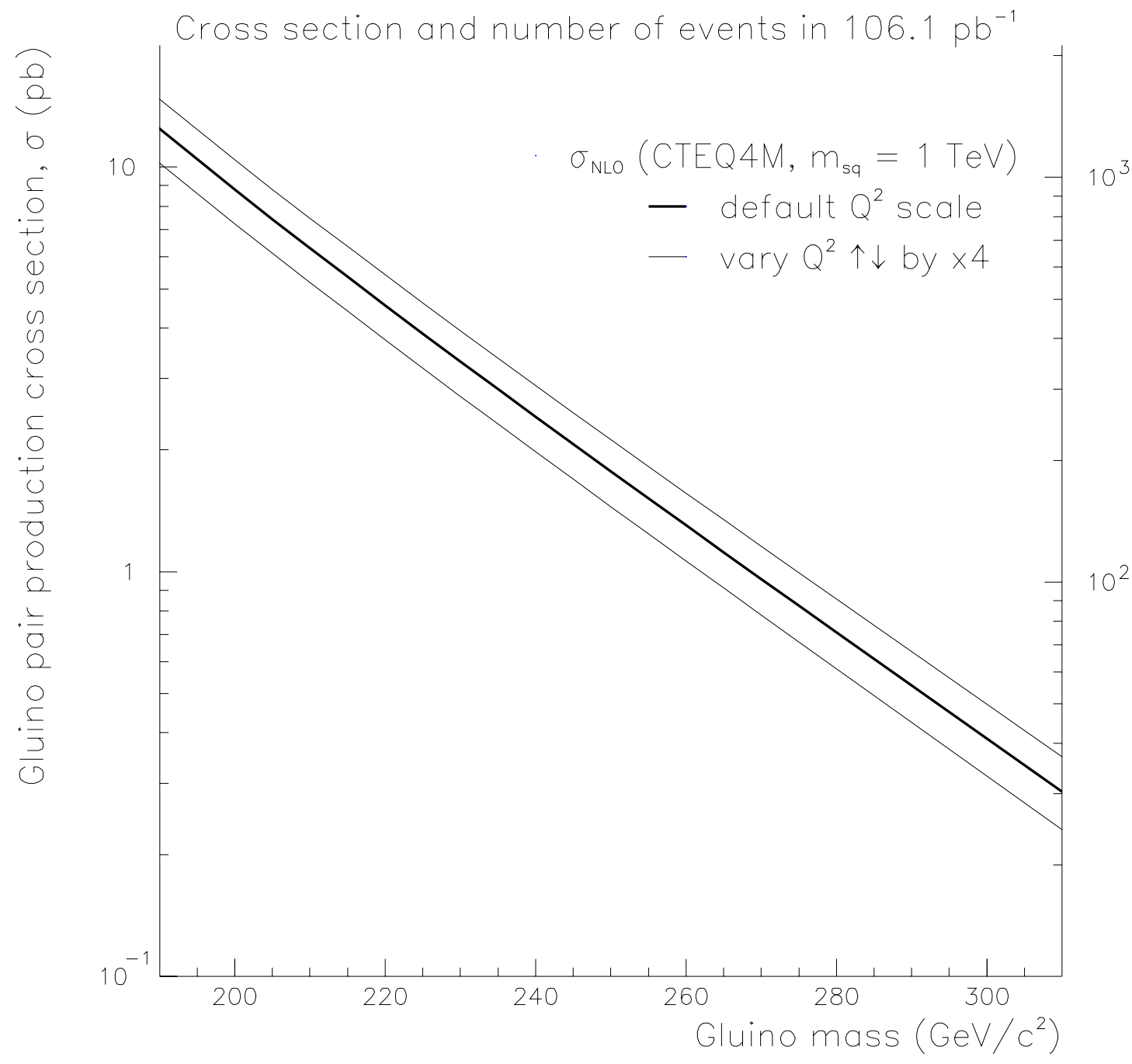

Figure 6.5: Calculation of $\tilde{g} \tilde{g}$ cross section (in pb) from PROSPINO. Values were calculated using the CTEQ 4M PDF and squark mass of $1 \mathrm{TeV}$. The central curve is for the default QCD renormalization scale, while the upper and lower come from increasing and decreasing, respectively, $Q^{2}$ by a factor of 4 . On the scale at right is the total number of $\tilde{g} \tilde{g}$ events expected for the Run I integrated luminosity. 
seen, the cross section for gluino production falls quickly with increasing gluino mass. Given that the accessible range of gluino masses is limited, relatively light decay products $(<100 \mathrm{GeV})$ will be required.

The mass hierarchy in Supersymmetry is determined by mixing of the various sparticle mass eigenstates to form observable states. In general, any scalar particles with the same electric charge, color quantum numbers, and $R$-parity may mix. Therefore, the squarks and sleptons of the MSSM are obtained by diagonalizing three $6 \times 6$ (mass $)^{2}$ matrices for the up-type squarks $\left(\tilde{u}_{L}, \tilde{c}_{L}, \tilde{t}_{L}, \tilde{u}_{R}, \tilde{c}_{R}, \tilde{t}_{R}\right)$, down-type squarks $\left(\tilde{d}_{L}, \tilde{s}_{L}, \tilde{b}_{L}, \tilde{d}_{R}, \tilde{s}_{R}, \tilde{b}_{R}\right)$, and charged sleptons $\left(\tilde{e}_{L}, \tilde{\mu}_{L}, \tilde{\tau}_{L}, \tilde{e}_{R}, \tilde{\mu}_{R}, \tilde{\tau}_{R}\right)$ and one $3 \times 3$ matrix for the sneutrinos $\left(\tilde{\nu}_{e}, \tilde{\nu}_{\mu}, \tilde{\nu}_{\tau}\right)$. Yet most of the mixing angles are very small. The third-generation squarks and sleptons benefit from large Yukawa couplings and may have significant mixing effects in pairs: $\left(\tilde{t}_{L}, \tilde{t}_{R}\right),\left(\tilde{b}_{L}, \tilde{b}_{R}\right)$, and $\left(\tilde{\tau}_{L}, \tilde{\tau}_{R}\right)$. The firstand second-generation sfermions have negligible Yukawa couplings and end up in the nearly degenerate pairs $\left(\tilde{e}_{R}, \tilde{\mu}_{R}\right),\left(\tilde{\nu}_{e}, \tilde{\nu}_{\mu}\right),\left(\tilde{e}_{L}, \tilde{\mu}_{L}\right),\left(\tilde{u}_{R}, \tilde{c}_{R}\right),\left(\tilde{d}_{R}, \tilde{s}_{R}\right),\left(\tilde{u}_{L}, \tilde{c}_{L}\right)$, and $\left(\tilde{d}_{L}, \tilde{s}_{L}\right)$.

Many searches for Supersymmetry assume a large degenerate squark mass for the first five pairs $(\tilde{u}, \tilde{d}, \tilde{s}, \tilde{c}, \tilde{b})$, while the scalar stop pair is allowed to mix freely:

$$
\left(\begin{array}{c}
\tilde{t}_{1} \\
\tilde{t}_{2}
\end{array}\right) \approx\left(\begin{array}{cc}
\cos \theta_{\tilde{t}} & \sin \theta_{\tilde{t}} \\
-\sin \theta_{\tilde{t}} & \cos \theta_{\tilde{t}}
\end{array}\right)\left(\begin{array}{c}
\tilde{t}_{L} \\
\tilde{t}_{R}
\end{array}\right)
$$

with $m_{\tilde{t}_{1}}^{2}<m_{\tilde{t}_{2}}^{2}$. Because the extent of sfermion mixing is proportional to the corresponding fermion mass, the effects can be quite pronounced owing to the large top mass, and $\tilde{t}_{1}$ is often presumed to be the lightest squark. For our purposes, we propose that $\tilde{t}_{1}$ is the only squark lighter than the gluino. Therefore, the preferred gluino decay mode is $\tilde{g} \rightarrow t \overline{\tilde{t}}$, assuming that $m_{\tilde{g}}>m_{t}+m_{\tilde{t}}$.

The stop has several decay options, depending on the relative masses of the lightest chargino $\left(\tilde{\chi}_{1}^{ \pm}\right)$, the sneutrino $(\tilde{\nu})$, and the lightest neutralino $\left(\tilde{\chi}_{1}^{0}\right)$ :

$$
\begin{aligned}
& \tilde{t} \longrightarrow b \tilde{\chi}_{1}^{ \pm} \\
& \tilde{t} \longrightarrow b l \tilde{\nu} \\
& \tilde{t} \longrightarrow c \tilde{\chi}_{1}^{0}
\end{aligned}
$$


For the potential light $\tilde{t}$ masses considered here, the chargino and neutralino are likely to be heavier and thus inaccessible to stop decay. The only available decay mode in this scenario is $\tilde{t} \longrightarrow c \tilde{\chi}_{1}^{0}$.

Motivated by the discussion above, we have attempted to simulate gluino pair production and the $\tilde{g} \rightarrow t \overline{\tilde{t}}$ decay in Monte Carlo. For our MC studies, we have selected ISAJET v7.37 and the CTEQ 3L [45] parton distribution function to generate samples for a wide array of gluino, stop, and neutralino masses. Figure 6.6 illustrates the available $m_{\tilde{g}}-m_{\tilde{t}}$ parameter space and the points that were generated in Monte Carlo.

The top quark mass was set equal to $175 \mathrm{GeV}$. Therefore we were limited to the kinematically allowed region $m_{\tilde{g}}-m_{\tilde{t}} \geq 175 \mathrm{GeV}$. For each gluino mass between 200 and $320 \mathrm{GeV}$, the corresponding stop mass was chosen such that $m_{\tilde{g}}-m_{\tilde{t}}=180,210$, or $240 \mathrm{GeV}$. For the majority of the generated samples, the neutralino mass was set to $\left(m_{\tilde{t}}-20 \mathrm{GeV}\right)$. In the region where $m_{\tilde{t}} \leq 40 \mathrm{GeV}$, stop-neutralino mass pairings of $\left(m_{\tilde{t}}, m_{\tilde{\chi}_{1}^{0}}\right)=(40,30),(30,20)$, and $(20,15) \mathrm{GeV}$ were selected to avoid unnaturally light neutralino masses.

For each of the indicated points in parameter space, Monte Carlo samples totaling 150,000 events were generated. All events were processed by the QFL' detector simulation and were subsequently filtered through the identical analysis code used for analyzing the Run I inclusive electron and inclusive muon datasets. The same-sign dilepton analysis is broken down into each of the event selection stages, and the full results are shown in Table 6.3. The number of events that pass each removal stage remains relatively uniform across all samples, at least within statistical uncertainties. For comparison purposes, a similar decomposition of an opposite-sign analysis applied to the same Monte Carlo samples is offered in Table 6.4.

In Table 6.5, we present the efficiencies for each selection stage in the analysis, averaged across the first 13 samples shown in Table 6.3. The efficiency for each filter is based only on those events which have passed all cuts up through the preceding stage. For comparison purposes, we have also shown the selection efficiencies for the opposite-sign analysis of a PYTHIA top 175 sample that was performed for the top dilepton analysis. Note that our results compare quite favorably. In fact, if the 


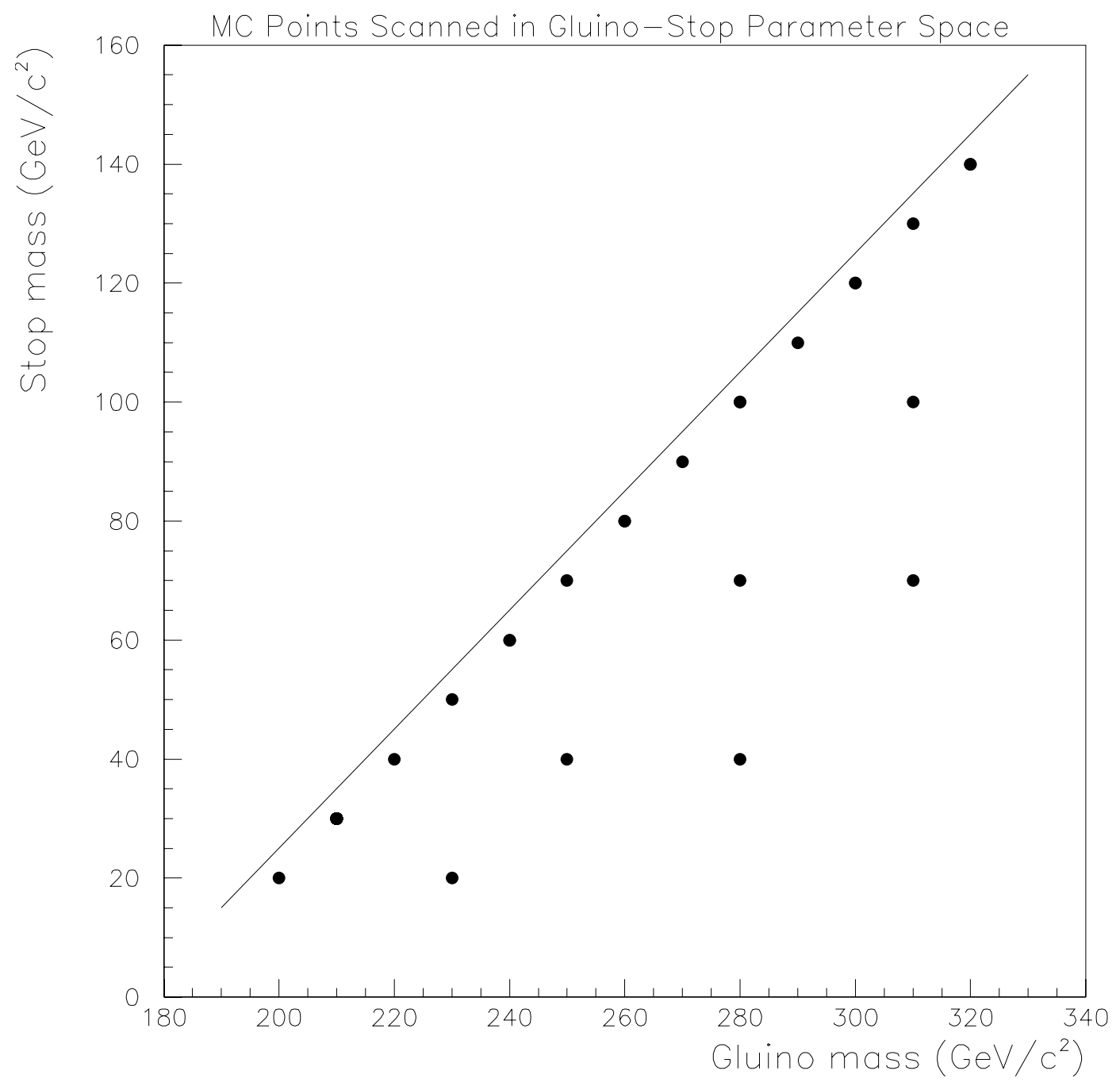

Figure 6.6: Combinations of gluino and stop mass explored in $\tilde{g} \rightarrow t \overline{\tilde{t}}$ Monte Carlo. The solid diagonal line indicates the kinematic limit on available stop masses due to the $175 \mathrm{GeV}$ top quark mass. The neutralino mass was generally related to the stop mass via $m_{\tilde{\chi}_{1}^{0}}=m_{\tilde{t}}-20 \mathrm{GeV}$, except where $m_{\tilde{t}} \leq 40 \mathrm{GeV}$. 


\begin{tabular}{|c|c|c|c|c|c|c|c|c|c|c|c|c|c|c|}
\hline \multicolumn{4}{|c|}{ MC SAMPLE INFO } & \multicolumn{11}{|c|}{ DILEPTON CUT } \\
\hline \multirow[t]{2}{*}{$m_{\tilde{g}}$} & \multirow[t]{2}{*}{$m_{\tilde{t}}$} & \multirow[t]{2}{*}{$m_{\tilde{\chi}_{1}^{0}}$} & \multirow{2}{*}{$\begin{array}{l}\text { No. of } \\
\text { Events }\end{array}$} & \multirow{2}{*}{$\begin{array}{l}\text { Lepton } \\
\text { ID }\end{array}$} & \multirow{2}{*}{$\begin{array}{c}\text { Cosmic } \\
\text { Ray }\end{array}$} & \multirow{2}{*}{$\begin{array}{l}\text { Same } \\
\text { Sign }\end{array}$} & \multirow[b]{2}{*}{ Isolation } & \multirow[b]{2}{*}{$M_{l l}$} & \multirow[b]{2}{*}{$E_{T}$} & \multirow[b]{2}{*}{$\Delta \phi\left(\mathbb{E}_{T}, l\right)$} & \multirow[b]{2}{*}{$\Delta \phi\left(\mathbb{E}_{T}, j\right)$} & \multicolumn{3}{|c|}{$N_{j}>10 \mathrm{GeV}$} \\
\hline & & & & & & & & & & & & 0 & 1 & $\geq 2$ \\
\hline 200 & 20 & 15 & 148483 & 2466 & 2465 & 1173 & 1098 & 960 & 844 & 805 & 760 & 3 & 80 & 677 \\
\hline 210 & 30 & 20 & 148165 & 2533 & 2531 & 1225 & 1132 & 958 & 839 & 803 & 747 & 5 & 70 & 672 \\
\hline 220 & 40 & 30 & 150000 & 2547 & 2546 & 1222 & 1139 & 974 & 847 & 790 & 736 & 6 & 65 & 665 \\
\hline 230 & 50 & 30 & 150000 & 2507 & 2503 & 1224 & 1118 & 952 & 838 & 798 & 745 & 1 & 46 & 698 \\
\hline 240 & 60 & 40 & 150000 & 2564 & 2564 & 1221 & 1111 & 950 & 842 & 800 & 742 & 3 & 30 & 709 \\
\hline 250 & 70 & 50 & 150000 & 2568 & 2565 & 1207 & 1097 & 971 & 857 & 811 & 743 & 0 & 21 & 722 \\
\hline 260 & 80 & 60 & 150000 & 2544 & 2542 & 1191 & 1096 & 958 & 875 & 838 & 767 & 1 & 35 & 730 \\
\hline 270 & 90 & 70 & 150000 & 2544 & 2540 & 1214 & 1116 & 973 & 854 & 807 & 739 & 3 & 31 & 705 \\
\hline 280 & 100 & 80 & 150000 & 2622 & 2620 & 1233 & 1123 & 963 & 858 & 816 & 745 & 1 & 23 & 720 \\
\hline 290 & 110 & 90 & 150000 & 2625 & 2625 & 1246 & 1130 & 990 & 864 & 831 & 771 & 0 & 38 & 733 \\
\hline 300 & 120 & 100 & 145579 & 2560 & 2557 & 1261 & 1145 & 986 & 882 & 836 & 780 & 3 & 39 & 738 \\
\hline 310 & 130 & 110 & 148992 & 2579 & 2577 & 1248 & 1133 & 981 & 884 & 826 & 760 & 0 & 28 & 732 \\
\hline 320 & 140 & 120 & 150000 & 2707 & 2706 & 1310 & 1201 & 1030 & 929 & 885 & 825 & 1 & 26 & 798 \\
\hline 230 & 20 & 15 & 148662 & 2494 & 2490 & 1204 & 1124 & 980 & 877 & 832 & 774 & 3 & 50 & 721 \\
\hline 250 & 40 & 30 & 148165 & 2580 & 2578 & 1245 & 1164 & 1007 & 922 & 885 & 825 & 0 & 37 & 788 \\
\hline 280 & 70 & 50 & 150000 & 2650 & 2647 & 1278 & 1157 & 1016 & 934 & 896 & 841 & 1 & 18 & 822 \\
\hline 310 & 100 & 80 & 148998 & 2579 & 2576 & 1254 & 1154 & 991 & 903 & 865 & 808 & 1 & 34 & 773 \\
\hline 280 & 40 & 30 & 147828 & 2532 & 2530 & 1251 & 1165 & 1001 & 912 & 888 & 829 & 2 & 27 & 800 \\
\hline 310 & 70 & 50 & 150000 & 2685 & 2685 & 1289 & 1177 & 1014 & 949 & 928 & 884 & 1 & 32 & 851 \\
\hline
\end{tabular}

Table 6.3: Results from like-sign analysis of Monte Carlo samples for $\tilde{g} \tilde{g} \rightarrow t t+X \rightarrow$ $l l+X$ for a range of gluino, stop, and neutralino masses. 


\begin{tabular}{|c|c|c|c|c|c|c|c|c|c|c|c|c|c|c|}
\hline \multicolumn{4}{|c|}{ MC SAMPLE INFO } & \multicolumn{11}{|c|}{ DILEPTON CUT } \\
\hline \multirow[t]{2}{*}{$m_{\tilde{g}}$} & \multirow[t]{2}{*}{$m_{\tilde{t}}$} & \multirow[t]{2}{*}{$m_{\tilde{\chi}_{1}^{0}}$} & \multirow{2}{*}{$\begin{array}{l}\text { No. of } \\
\text { Events }\end{array}$} & \multirow{2}{*}{$\begin{array}{l}\text { Lepton } \\
\text { ID }\end{array}$} & \multirow{2}{*}{$\begin{array}{c}\text { Cosmic } \\
\text { Ray }\end{array}$} & \multirow{2}{*}{$\begin{array}{l}\text { Opp. } \\
\text { Sign }\end{array}$} & \multirow[b]{2}{*}{ Isolation } & \multirow[b]{2}{*}{$M_{l l}$} & \multirow[b]{2}{*}{$\mathbb{E}_{T}$} & \multirow[b]{2}{*}{$\Delta \phi\left(\mathbb{E}_{T}, l\right)$} & \multirow[b]{2}{*}{$\Delta \phi\left(\mathbb{E}_{T}, j\right)$} & \multicolumn{3}{|c|}{$N_{j}>10 \mathrm{GeV}$} \\
\hline & & & & & & & & & & & & 0 & 1 & $\geq 2$ \\
\hline 200 & 20 & 15 & 148483 & 2466 & 2465 & 1305 & 1207 & 1044 & 929 & 869 & 799 & 8 & 84 & 707 \\
\hline 210 & 30 & 20 & 148165 & 2533 & 2531 & 1317 & 1215 & 1052 & 912 & 872 & 790 & 2 & 54 & 734 \\
\hline 220 & 40 & 30 & 150000 & 2547 & 2546 & 1333 & 1218 & 1051 & 938 & 885 & 823 & 2 & 65 & 756 \\
\hline 230 & 50 & 30 & 150000 & 2507 & 2503 & 1293 & 1154 & 999 & 880 & 843 & 788 & 0 & 36 & 752 \\
\hline 240 & 60 & 40 & 150000 & 2564 & 2564 & 1355 & 1219 & 1043 & 915 & 861 & 771 & 2 & 28 & 740 \\
\hline 250 & 70 & 50 & 150000 & 2568 & 2565 & 1374 & 1228 & 1053 & 935 & 879 & 811 & 0 & 38 & 772 \\
\hline 260 & 80 & 60 & 150000 & 2544 & 2542 & 1366 & 1218 & 1039 & 896 & 850 & 782 & 1 & 34 & 747 \\
\hline 270 & 90 & 70 & 150000 & 2544 & 2540 & 1343 & 1205 & 1037 & 920 & 869 & 794 & 1 & 42 & 751 \\
\hline 280 & 100 & 80 & 150000 & 2622 & 2620 & 1395 & 1262 & 1074 & 967 & 915 & 845 & 2 & 41 & 802 \\
\hline 290 & 110 & 90 & 150000 & 2625 & 2625 & 1391 & 1245 & 1055 & 929 & 888 & 818 & 0 & 30 & 788 \\
\hline 300 & 120 & 100 & 145579 & 2560 & 2557 & 1312 & 1206 & 1051 & 935 & 905 & 831 & 2 & 41 & 788 \\
\hline 310 & 130 & 110 & 148992 & 2579 & 2577 & 1352 & 1208 & 1011 & 884 & 837 & 771 & 2 & 32 & 737 \\
\hline 320 & 140 & 120 & 150000 & 2707 & 2706 & 1403 & 1267 & 1097 & 988 & 944 & 869 & 2 & 40 & 827 \\
\hline 230 & 20 & 15 & 148662 & 2494 & 2490 & 1301 & 1187 & 1040 & 942 & 902 & 836 & 2 & 53 & 781 \\
\hline 250 & 40 & 30 & 148165 & 2580 & 2578 & 1345 & 1210 & 1046 & 954 & 914 & 847 & 1 & 38 & 808 \\
\hline 280 & 70 & 50 & 150000 & 2650 & 2647 & 1379 & 1250 & 1090 & 991 & 965 & 884 & 2 & 20 & 862 \\
\hline 310 & 100 & 80 & 148998 & 2579 & 2576 & 1337 & 1211 & 1058 & 971 & 934 & 864 & 0 & 34 & 830 \\
\hline 280 & 40 & 30 & 147828 & 2532 & 2530 & 1290 & 1174 & 1015 & 919 & 892 & 840 & 2 & 30 & 808 \\
\hline 310 & 70 & 50 & 150000 & 2685 & 2685 & 1402 & 1279 & 1122 & 1059 & 1022 & 965 & 0 & 14 & 948 \\
\hline
\end{tabular}

Table 6.4: Results from opposite-sign analysis of Monte Carlo samples for $\tilde{g} \tilde{g} \rightarrow$ $t t+X \rightarrow l l+X$ for a range of gluino, stop, and neutralino masses. 


\begin{tabular}{|l|c|c|}
\hline \hline \multirow{2}{*}{ Event selection cut } & \multicolumn{2}{|c|}{ Filter efficiency } \\
\cline { 2 - 3 } Lepton ID & $\tilde{g} \tilde{g}(\mathrm{SS})$ & $t \bar{t}(\mathrm{OS})$ \\
Cosmic ray filter & 0.0172 & 0.0150 \\
SS/OS dilepton pair & 0.999 & 0.999 \\
Isolation & 0.479 & 0.950 \\
$Z$ mass filter & 0.917 & 0.949 \\
$\mathbb{E}_{T}, \Delta \phi\left(\mathbb{E}_{T}, j\right), \Delta \phi\left(\mathbb{E}_{T}, j\right)$ & 0.864 & 0.880 \\
$n_{\text {jets }} \geq 2$ & 0.940 & 0.755 \\
\hline Total dilepton acceptance & 0.0479 & 0.0762 \\
\hline \hline
\end{tabular}

Table 6.5: Comparison of the selection efficiencies in Monte Carlo for our $\tilde{g} \tilde{g}$ samesign dilepton analysis versus an opposite-sign analysis of PYTHIA $t \bar{t}$ events. Each efficiency is based on the number of events which have passed all of the preceding cuts. The difference in overall acceptance is due mainly to the branching ratio of $\tilde{g} \tilde{g}$ to like-sign top.

loss of half of all $\tilde{g} \tilde{g}$ events to the same-sign dilepton cut is factored out, the overall acceptance rate is significantly better than for $t \bar{t}$ events. Also note that there are no forced decays of the top quarks in either Monte Carlo. Therefore, the branching ratio of $(t t, t \bar{t} \rightarrow l l+X)$ is included in the lepton ID efficiency.

\subsection{Lepton ID efficiencies}

Determination of the acceptance is unfortunately not as simple and straightforward as generating a large number of events and counting the percentage which pass all of the selection cuts in the analysis. Despite the massive collaborative effort 
invested into the design and implementation of Monte Carlo generators and detector simulations such as QFL', these tools are inexact and do not reproduce every aspect of event topology with full accuracy. In particular, differences have been observed between the data and Monte Carlo modeling of lepton identification variables. Systematic studies have been performed to determine the extent of the lepton ID efficiency mismatch, and correction factors are now available for scaling Monte Carlo results for comparison with data.

The procedure used to determine the lepton ID efficiencies is fully characterized in Ref. [36]. The ID efficiencies for data were calculated using $Z^{0} \rightarrow l l$ events in Run Ib, and Monte Carlo efficiencies were measured using a 100,000 event ISAJET sample of $Z^{0} \rightarrow l l$ combined with the QFL detector simulation package. A narrow dilepton invariant mass window of $80<M_{l l}<100 \mathrm{GeV}$ about the $Z^{0}$ mass was used to select events for both calculations. For each type of lepton (TCE, TCM, etc.), the efficiency shown here is the probability that a lepton with transverse energy or momentum $>20 \mathrm{GeV}$ which passes the fiducial requirements of Sections 4.1.1 and 4.2.1 will pass the full set of lepton ID cuts. A summary of the results is presented in Table 6.6.

The lepton ID efficiency is sensitive to the underlying event activity. Contamination of the kinematic variables occurs due to spurious tracks and calorimeter energy within the cone about the lepton direction. Therefore the ID efficiency is affected by the source. Leptons from $Z^{0}$ decay differ somewhat from those in $t \bar{t}$ events, where the lepton may originate from $W^{ \pm}, \tau$, bottom, or charm. $Z^{0}$ dilepton events are comparatively devoid of jet activity, whose effect on lepton ID cannot be neglected. The $Z^{0} \rightarrow l l$ Monte Carlo also does not accurately reproduce the existing jet activity observed in the data.

To compensate for these effects, the lepton ID efficiencies for data and Monte Carlo have been remeasured after splitting the leptons into 3 separate bins of calorimeter isolation: $0<I_{\text {cal }}<0.02,0.02<I_{\text {cal }}<0.1$, and $I_{\text {cal }}>0.1$. Because isolation is built into the definition of minimum ionizing muons (CMI), the detection efficiency was not divided according to isolation. The efficiencies presented here are determined after the isolation requirement has been applied to each event, so the numbers are valid for 


\begin{tabular}{|c|cc|}
\hline \hline \multirow{2}{*}{ Lepton Type } & Lepton ID Efficiencies \\
\cline { 2 - 3 } TCE & $0.818 \pm 0.009$ & $0.892 \pm 0.003$ \\
LCE & $0.889 \pm 0.007$ & $0.936 \pm 0.003$ \\
TCM & $0.922 \pm 0.009$ & $0.978 \pm 0.002$ \\
CMX & $0.914 \pm 0.010$ & $0.972 \pm 0.002$ \\
CMI & $0.913 \pm 0.013$ & $0.962 \pm 0.003$ \\
\hline \hline
\end{tabular}

Table 6.6: Comparison of lepton ID efficiencies for $Z^{0} \rightarrow l l$ Run Ib data and ISAJET+QFL Monte Carlo for the five lepton classes. The errors are statistical only.

our analysis after all selection cuts (including isolation) have been made. The largest discrepancies between data and Monte Carlo occur in the highest isolation bin, which mostly affects leptons from semileptonic $b$ and $c$ decays. The results are summarized in Table 6.7 and Figure 6.7.

This approach does not directly address the dependency of the efficiency on the parent particle which produces the lepton. Leptons from $W^{ \pm}$decay in top events share a similar source of non-isolation with leptons from $Z^{0} \rightarrow l l$ events: additional tracks from uncorrelated jets within the isolation cone. On the other hand, leptons from $b$ decay are fundamentally different, with the lepton surrounded by jet activity from $b$ jet. Since the majority of leptons in $t \bar{t}$ events originate from $W$ decay, the scale factors derived here provide a good approximation to all top events. The systematic error attributed to this simplification will be addressed in Section 7.6.2.

In our Monte Carlo simulation of $\tilde{g} \tilde{g} \rightarrow l^{ \pm} l^{ \pm}+X$, the final step in calculating the acceptance is to apply the scale factors shown in Table 6.8. In each event, the isolation is calculated for every lepton which passes the ID requirements. The appropriate scale factor is assigned to each lepton according to the type and isolation. If an event passes 


\begin{tabular}{|cc|c|c|c|}
\hline \hline $\begin{array}{c}\text { Lepton } \\
\text { category }\end{array}$ & Source & $I_{\text {cal }}<0.02$ & $0.02<I_{\text {cal }}<0.1$ & $I_{\text {cal }}>0.1$ \\
\hline TCE & MC & 0.893 & 0.884 & 0.840 \\
& data & 0.839 & 0.810 & 0.576 \\
\hline LCE & MC & 0.937 & 0.936 & 0.889 \\
& data & 0.912 & 0.882 & 0.606 \\
\hline TCM & MC & 0.979 & 0.976 & 0.902 \\
& data & 0.934 & 0.925 & 0.645 \\
\hline CMX & MC & 0.973 & 0.962 & 0.972 \\
& data & 0.923 & 0.897 & 0.880 \\
\hline
\end{tabular}

\begin{tabular}{|cc|c|}
\hline $\begin{array}{c}\text { Lepton } \\
\text { category }\end{array}$ & Source & $I_{\text {cal }}<0.1$ \\
\hline CMI & MC & 0.962 \\
& data & 0.913 \\
\hline \hline
\end{tabular}

Table 6.7: Comparison of lepton ID efficiencies in Monte Carlo versus data as a function of calorimeter isolation for the TCE, LCE, TCM, and CMX lepton classes. For minimum ionizing muons (CMI), all allowed isolation values are grouped together. 


\section{Lepton ID Efficiencies as a Function of Isolation}
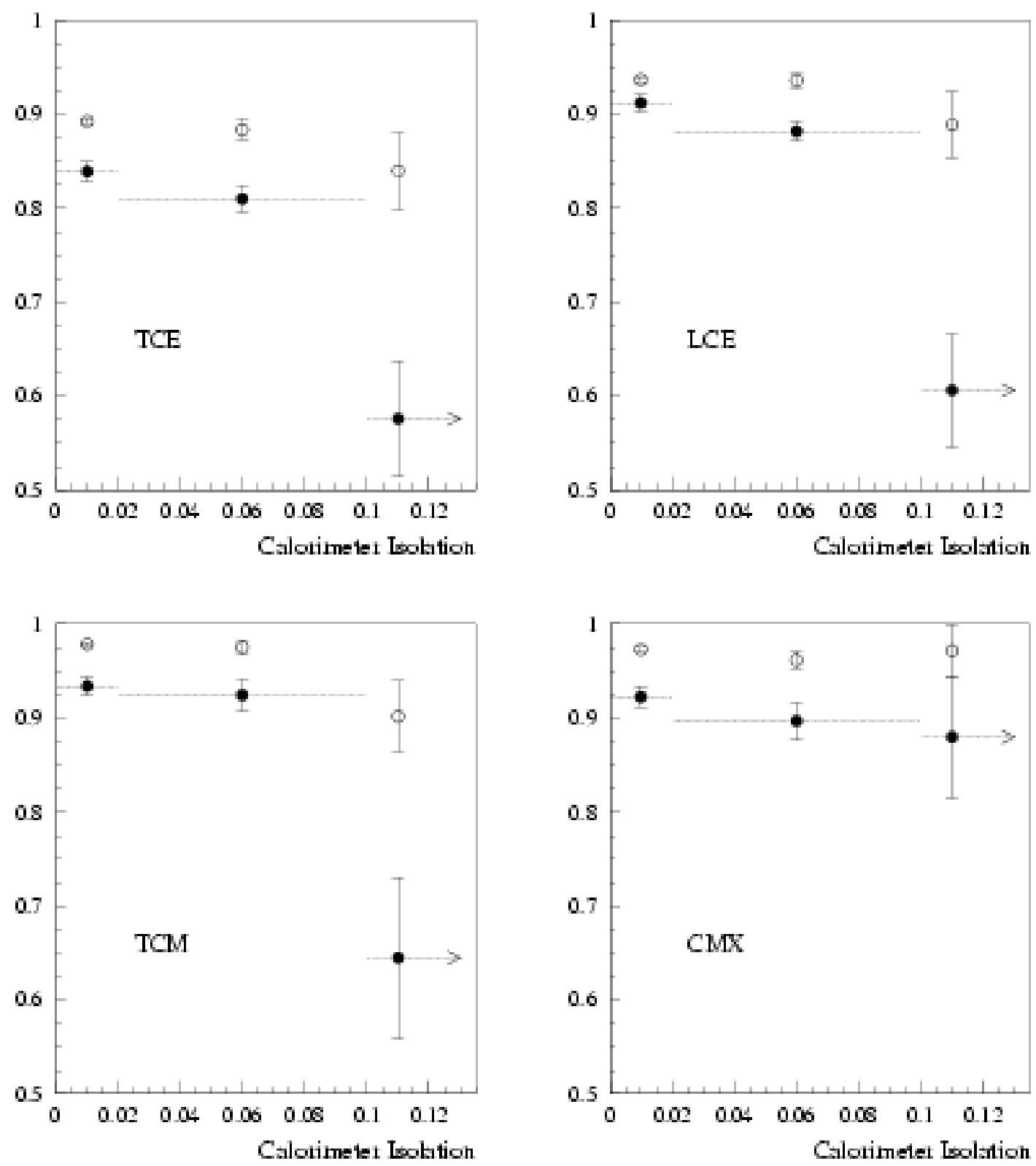

Figure 6.7: Lepton ID efficiencies for the lepton classes TCE, LCE, TCM, and CMX for three ranges of calorimeter isolation. The open points are $Z^{0} \rightarrow l l$ Monte Carlo and the closed points are data. The dashed lines show the 3 isolation bins, and the error bars are statistical only. 


\begin{tabular}{|c|c|c|c|}
\hline \hline \multirow{2}{*}{$\begin{array}{c}\text { Lepton } \\
\text { category }\end{array}$} & \multicolumn{3}{|c|}{ Scale factor, $\epsilon_{I D}($ data $) / \epsilon_{I D}(M C)$} \\
\cline { 2 - 4 } & $I_{\text {cal }}<0.02$ & $0.02<I_{\text {cal }}<0.1$ & $I_{\text {cal }}>0.1$ \\
\hline TCE & 0.940 & 0.916 & 0.686 \\
\hline LCE & 0.973 & 0.942 & 0.682 \\
\hline TCM & 0.954 & 0.948 & 0.715 \\
\hline CMX & 0.949 & 0.932 & 0.905 \\
\hline CMI & \multicolumn{3}{|c}{0.949} \\
\hline \hline
\end{tabular}

Table 6.8: Scale factors for correcting the same-sign dilepton event acceptance to account for differences in lepton ID efficiencies between Run I data and Monte Carlo. For minimum ionizing muons (CMI), all allowed isolation values are grouped together, while all other lepton classes are separated into 3 isolation bins.

all selection cuts in the analysis, it is weighted by the product of the two scale factors for the dilepton pair. (In the case of a trilepton event, the scale factors of the leptons in the first valid same-sign dilepton pair identified are used.) The overall effect of the correction factor is a reduction in the event acceptance of $\sim 15.6 \%$. (In the top dilepton analysis, the scale factors reduced the acceptance by $13 \%$.)

Table 6.9 shows the results of applying the lepton ID correction factors to the output of our $\tilde{g} \tilde{g}$ Monte Carlo samples. The acceptance rate is also calculated for each pairing of $m_{\tilde{g}}$ and $m_{\tilde{t}}$. Finally, in Figure 6.8 we plot the cumulative efficiency for each filter stage in the same-sign analysis of the first $13 \tilde{g} \tilde{g}$ Monte Carlo samples. 


\begin{tabular}{|c|c|c|c|c|c|c|}
\hline \multicolumn{4}{|c|}{ MC SAMPLE INFO } & \multirow{2}{*}{$\begin{array}{c}\# \text { of events } \\
\text { passing } \\
\text { LS analysis }\end{array}$} & \multirow{2}{*}{$\begin{array}{l}\text { \# of events } \\
\text { scaled by } \\
\text { lep ID eff }\end{array}$} & \multirow{2}{*}{$\begin{array}{c}\text { Event } \\
\text { acceptance } \\
\text { rate }\end{array}$} \\
\hline$m_{\tilde{g}}$ & $m_{\tilde{t}}$ & $m_{\tilde{\chi}_{1}^{0}}$ & $\begin{array}{l}\text { No. of } \\
\text { Events }\end{array}$ & & & \\
\hline 200 & 20 & 15 & 148483 & 677 & 582.3 & 0.00392 \\
\hline 210 & 30 & 20 & 148165 & 672 & 566.2 & 0.00382 \\
\hline 220 & 40 & 30 & 150000 & 665 & 563.2 & 0.00375 \\
\hline 230 & 50 & 30 & 150000 & 698 & 585.2 & 0.00390 \\
\hline 240 & 60 & 40 & 150000 & 709 & 596.3 & 0.00398 \\
\hline 250 & 70 & 50 & 150000 & 722 & 608.0 & 0.00405 \\
\hline 260 & 80 & 60 & 150000 & 730 & 615.4 & 0.00410 \\
\hline 270 & 90 & 70 & 150000 & 705 & 594.9 & 0.00397 \\
\hline 280 & 100 & 80 & 150000 & 720 & 609.4 & 0.00406 \\
\hline 290 & 110 & 90 & 150000 & 733 & 616.3 & 0.00411 \\
\hline 300 & 120 & 100 & 145579 & 738 & 617.2 & 0.00424 \\
\hline 310 & 130 & 110 & 148992 & 732 & 617.1 & 0.00414 \\
\hline 320 & 140 & 120 & 150000 & 798 & 674.9 & 0.00450 \\
\hline 230 & 20 & 15 & 148662 & 721 & 617.0 & 0.00415 \\
\hline 250 & 40 & 30 & 148165 & 788 & 669.0 & 0.00452 \\
\hline 280 & 70 & 50 & 150000 & 822 & 693.6 & 0.00462 \\
\hline 310 & 100 & 80 & 148998 & 773 & 650.2 & 0.00436 \\
\hline 280 & 40 & 30 & 147828 & 800 & 676.8 & 0.00458 \\
\hline 310 & 70 & 50 & 150000 & 851 & 717.4 & 0.00478 \\
\hline
\end{tabular}

Table 6.9: Results of the same-sign analysis of all $\tilde{g} \tilde{g}$ Monte Carlo samples after application of the event-by-event scale factors to correct for the differences in lepton ID efficiency between MC and data. The overall acceptance rate, including the scale factors, is calculated in the column at right. 


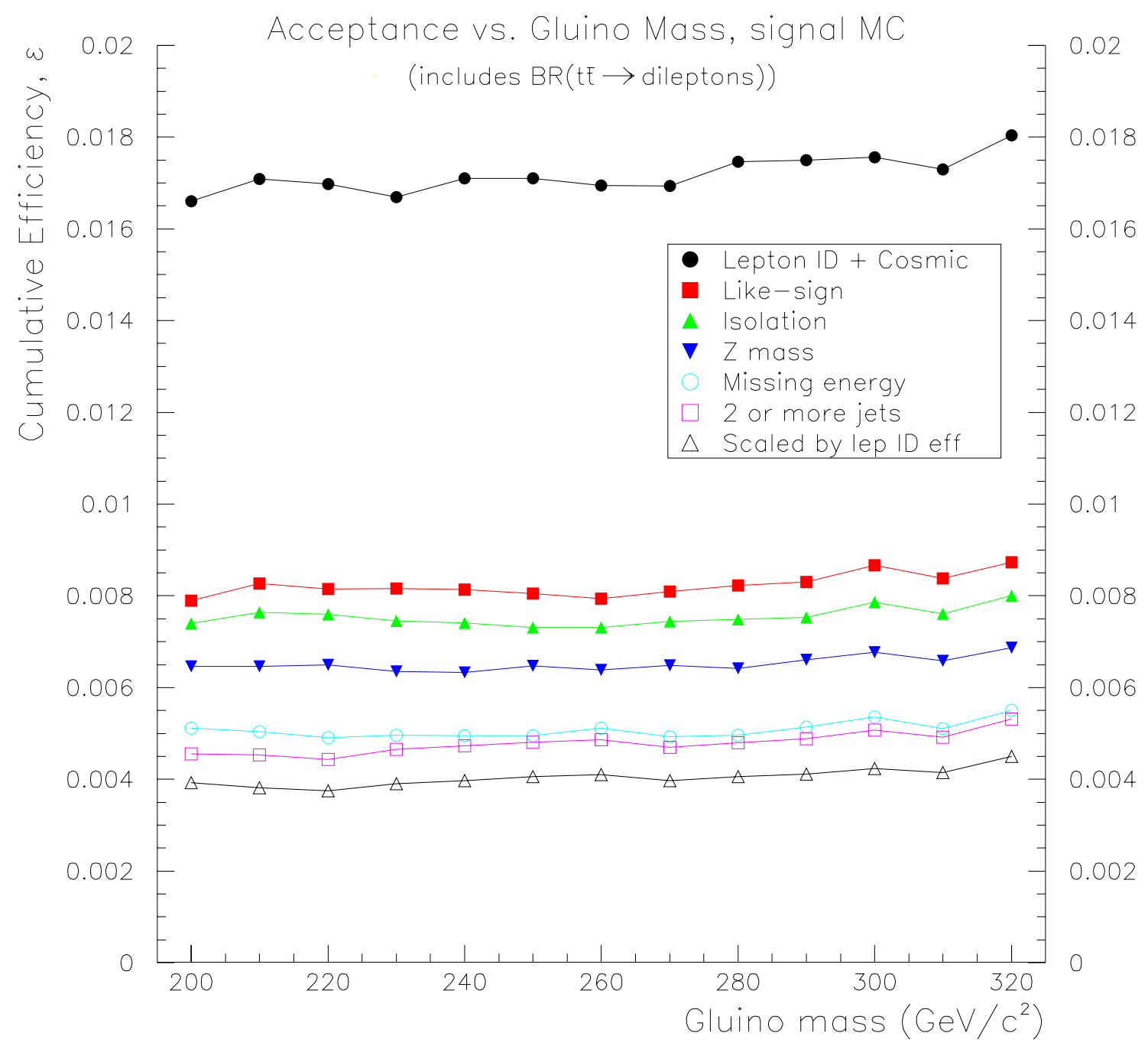

Figure 6.8: Cumulative efficiency at each stage of the same-sign dilepton analysis for $\tilde{g} \rightarrow t \overline{\tilde{t}}$ events in Monte Carlo. These results cover only the samples where $200 \leq m_{\tilde{g}} \leq 320, m_{\tilde{t}}=m_{\tilde{g}}-180 \mathrm{GeV}$ and $m_{\tilde{\chi}_{1}^{0}}=m_{\tilde{t}}-20 \mathrm{GeV}$ (except for $m_{\tilde{t}} \leq 40$ $\mathrm{GeV})$. 


\begin{tabular}{|c|c|c|c|c|}
\hline \hline \multirow{2}{*}{} & \multicolumn{4}{|c|}{ Acceptance rates } \\
\cline { 2 - 5 } & \multicolumn{2}{|c|}{ Before scaling } & \multicolumn{2}{c|}{ After scaling } \\
\cline { 2 - 5 } & Range & Average & Range & Average \\
\hline All 19 samples & $0.443-0.567 \%$ & $0.496 \%$ & $0.375-0.478 \%$ & $0.419 \%$ \\
First 13 samples & $0.443-0.532 \%$ & $0.479 \%$ & $0.375-0.450 \%$ & $0.404 \%$ \\
\hline \hline
\end{tabular}

Table 6.10: Summary of the same-sign dilepton acceptance rates calculated from Monte Carlo simulation of $\tilde{g} \rightarrow t \overline{\tilde{t}}$.

\subsection{Same-sign dilepton acceptance summary}

After an extensive analysis of $\tilde{g} \tilde{g}$ Monte Carlo for a variety of gluino and stop masses, we have determined the overall acceptance rate in the same-sign dilepton channel for $\tilde{g} \rightarrow t \overline{\tilde{t}}$ events. In Table 6.10, we have summarized the acceptance calculation for the Monte Carlo samples listed in Table 6.9. A separate set of results for the first $13 \mathrm{MC}$ samples is also provided. After determination of the systematic error on the acceptance and the expected background contribution to the same-sign dilepton channel, calculation of the cross-section limit and any potential exclusion of gluino mass will be based upon the smaller collection of 13 samples. 


\section{Chapter 7}

\section{Systematic Uncertainty}

After simulating the gluino production and decay process in Monte Carlo and calculating the like-sign dilepton channel acceptance, the next step is to determine the confidence level of the Monte Carlo simulation. Multiple factors influence the calculation, especially when studying a statistics-limited process. Any assumptions potentially skew the results, and we shall attempt to quantify the error in our measurement of the acceptance in the following sections.

This analysis shares many of the same sources of systematic uncertainty with the top dilepton analysis. In fact, any systematic errors for that analysis which were previously calculated using the data are directly applicable since none of the event selection procedures have been significantly changed for this search. First, we shall give those that were recalculated using our Monte Carlo, then we will briefly describe those that are carried over from the top dilepton analysis.

Ideally, the systematic error should be calculated for several, if not all, of the signal MC points generated. This would be important if the analysis claimed to exclude a large portion of the available parameter space. Since we knew that the exclusion region (if any) would be located near the lower range of gluino masses, we chose the $\left(m_{\tilde{g}}, m_{\tilde{t}}, m_{\tilde{\chi}_{1}^{0}}\right)=(210,30,20) \mathrm{GeV}$ mass point for determining the systematic errors. The systematic error should be representative of that for the other Monte Carlo points. 


\subsection{Parton distribution function}

The parton distribution function (PDF) models the internal structure of the proton and antiproton. These structure functions determine the momentum distribution of the partons (the quark constituents and the virtual gluons exchanged between them), and this affects the ability of the reaction to produce the heavy final state products we are studying.

For the signal MC samples, we used CTEQ 3L, which is a leading order (LO) parton distribution function. We were interested in trying higher order PDFs, so we chose one from the GRV family (GRV $94 \mathrm{HO}$ [46]) and one from the MRS family (MRSD0' [47]). The samples were regenerated using these distribution functions, and the resulting acceptances are shown in Table 7.1. The largest difference (6.8\%) is between GRV $94 \mathrm{HO}$ and CTEQ 3L, yet the values remain consistent within statistical errors. We assign half of this value (3.4\%) to be the uncertainty due to PDF choice.

\begin{tabular}{|c|c|c|}
\hline$\epsilon_{d i l}=(0.382 \pm 0.016) \%$ & CTEQ 3L & LO \\
\hline$\epsilon_{d i l}=(0.402 \pm 0.016) \%$ & MRSD0' & NLL \\
\hline$\epsilon_{d i l}=(0.408 \pm 0.017) \%$ & GRV $94 \mathrm{HO}$ & NLL \\
\hline
\end{tabular}

Table 7.1: Dependence of acceptance on choice of PDF

\subsection{Initial and final state gluon radiation}

Monte Carlo fails to accurately reproduce certain aspects of gluon radiation which are observed in data. This particularly affects event signatures which are dependent upon counting jets or placing requirements on lepton isolation. In the case of $\tilde{g} \rightarrow t \overline{\tilde{t}}$ production, fragmentation of the two $b$ quarks from top produces sufficient jet activity to meet the $N_{j e t} \geq 2$ cut. Any additional contribution to the jet multiplicity from gluon radiation is unlikely to significantly increase the dilepton acceptance. The 
extra underlying jet activity counteractively serves to reduce the efficiency of the lepton isolation cut. These competing effects from gluon radiation make prediction of the net impact on the acceptance difficult.

The default behavior of ISAJET is to include both initial and final state radiation. We can estimate the overall influence of ISR and FSR simulation by alternately switching them on and off in the MC generator. Initial state radiation is suppressed by simply equating the QCD scale factor, $L A M B D A$ in the ISAJET parameter file, with the beam energy. Final state radiation is controlled by setting the jet energy cutoff for secondary particle creation, CUTJET, to coincide with the beam energy and then restoring the original cutoff after ISAJET has performed the final state jet evolution. Both of these steps may be performed simultaneously to remove both IS and FS radiation from the simulation.

Our method of estimating the error due to gluon radiation involves turning off final-state splitting while leaving on initial-state (IS on, FS off) and vice-versa (IS off, FS on), and then recalculating the acceptance for both cases. Table 7.2 shows the results. In both cases, the acceptance increases over the default setting, with the maximum difference being $6.5 \%$. Once again, the variation is within statistical error, so we use half the difference $(3.3 \%)$ as the systematic error for gluon radiation simulation.

\begin{tabular}{rl|}
\hline \hline$\epsilon_{d i l}=(0.382 \pm 0.016) \%$ & IS \& FS radiation on (default) \\
$\epsilon_{d i l}=(0.407 \pm 0.016) \%$ & IS radiation on, FS radiation off \\
$\epsilon_{d i l}=(0.405 \pm 0.016) \%$ & IS radiation off, FS radiation on \\
\hline \hline
\end{tabular}

Table 7.2: Dependence of acceptance on IS and FS gluon radiation 


\section{3 $\quad Q^{2}$ scale dependence}

In proton-antiproton collisions, the strength of the interaction is determined in part by the momentum transferred between the reacting partons within $p$ and $\bar{p}$. This is reflected in the "running," or energy dependence, of the strong coupling constant, $\alpha_{s}$.

In QED, the fine structure constant also changes with the momentum transfer, although more slowly and in an increasing manner. The bare charge of the electron is shielded from the external world by the creation of virtual electron-positron pairs from the energy of the vacuum. To an outside test charge, the effective charge of the electron is reduced due to this screening effect. If the test charge energy is increased and it more closely approaches the electron, the apparent electron charge increases, as does the strength of the interaction.

The opposite phenomenon occurs for particles with color charge in QCD. In addition to the contribution from quark-antiquark pairs, the gluons exchanging color information interact with each other and provide an antiscreening effect. At higher energies, or large momentum transfers, the effective color charge of the target quark and the interaction strength are reduced. The dependence of $\alpha_{s}$ is logarithmic in $Q$, so it is not necessary to exactly determine the momentum transfer in each reaction, even to within a factor of two in uncertainty.

In ISAJET, all lowest order QCD processes which give rise to two high- $p_{T}$ jets, such as $g+g \rightarrow g+g$ and $g+g \rightarrow q+\bar{q}$, are governed by the two-body to twobody interaction. This process is most easily described using the Lorentz-invariant kinematic quantities known as Mandelstam variables. If the incident (final) momenta are $p$ and $p^{\prime}\left(k\right.$ and $\left.k^{\prime}\right)$, these are defined as:

$$
\begin{aligned}
& \hat{s}=\left(p+p^{\prime}\right)^{2}=\left(k+k^{\prime}\right)^{2} \\
& \hat{t}=(k-p)^{2}=\left(k^{\prime}-p^{\prime}\right)^{2} \\
& \hat{u}=\left(k^{\prime}-p\right)^{2}=\left(k-p^{\prime}\right)^{2} .
\end{aligned}
$$

The $Q^{2}$ scale employed by ISAJET is a function of the Mandelstam quantities:

$$
Q^{2}=\frac{2 \hat{s} \hat{t} \hat{u}}{\hat{s}^{2}+\hat{t}^{2}+\hat{u}^{2}}
$$




\begin{tabular}{rlrl}
\hline \hline$\epsilon_{d i l}$ & $=(0.398 \pm 0.016) \%$ & & decrease $Q^{2} \times 4$ \\
$\epsilon_{d i l}$ & $=(0.382 \pm 0.016) \%$ & & normal $Q^{2}$ scale \\
$\epsilon_{d i l}$ & $=(0.396 \pm 0.016) \%$ & & increase $Q^{2} \times 4$ \\
\hline \hline
\end{tabular}

Table 7.3: Dependence of acceptance on the choice of the QCD momentum transfer scale.

The dependence of the like-sign dilepton acceptance on the chosen momentum transfer scale is determined by raising and lowering $Q^{2}$ by a multiplicative factor of 4. For each new $Q^{2}$ scale, the Monte Carlo set was regenerated, and the results of the analysis are shown in Table 7.3. The variation in acceptance is small (4.2\%) and is well within the statistical error. Therefore, we assign half the difference $(2.1 \%)$ to be the systematic error due to the choice of $Q^{2}$ scale in ISAJET.

\subsection{Jet energy scale dependence}

As described in Section 4.9, several factors contribute to the uncertainty of the jet energy measurement. The first issue concerns the accurate portrayal of the detector response, which ties into the proper tuning of the QFL' detector simulation. Several CDF notes [48, 49] detail the "Behrends curve," which was used in the top mass analysis to assign the systematic uncertainty due to detector effects. The curve allows one to calculate the uncertainty when comparing uncorrected jet energies in data and Monte Carlo. For a given in-cone energy, the error varies from $10 \%$ at $8 \mathrm{GeV}$ to $3 \%$ at $100 \mathrm{GeV}$, plus an additional $2 \%$ error to account for changes in scale between Run I and the 1989 run when the Behrends curve was calculated.

A larger error arises when considering out-of-cone energy loss. For example, if a final state quark radiates a gluon outside the cone size of an analysis, the jet energy is reduced . A study [50] of the energy distribution inside varying cone sizes using photon data and Monte Carlo revealed a possible $10 \%$ shift for the energy measurement. 


\begin{tabular}{rlr}
\hline \hline$\epsilon_{d i l}=(0.376 \pm 0.016) \%$ & decrease jet energy scale $\times 5 \%$ \\
$\epsilon_{d i l}=(0.382 \pm 0.016) \%$ & default scale \\
$\epsilon_{d i l}=(0.389 \pm 0.016) \%$ & increase jet energy scale $\times 5 \%$ \\
\hline \hline
\end{tabular}

Table 7.4: Dependence of acceptance on jet energy scale

The accepted practice of the top group has been to assign a $10 \%$ systematic for the jet energy scale [53]. Attempts have been made to further understand this effect and reduce the systematic uncertainty for gluon radiation [51, 52]. Many exotics analyses adopt a 5\% systematic error. One should assume that the measured jet energies are either under- or overestimated by this amount and vary the jet energies in Monte Carlo to determine what effect this has on the acceptance calculation.

In the analysis, there are three areas where the jet energy scale has an impact: the $E_{T}^{\text {raw }}$ requirement for jet ID, the corrections made to the missing energy using the difference between raw and corrected jet energies, and calorimeter isolation cut for leptons. Using the original MC events which have passed through the QFL' detector simulation, the jet energies can be effectively increased or decreased by simply lowering or raising the energy cut used for jet ID by factors of $\frac{1}{1.05}$ and $\frac{1}{0.95}$. The $\mathbb{E}_{T}$ correction routine has hooks built in for changing the jet energy scale, so only the appropriate flags need be set to perform the correction. Finally, the calorimeter isolation values for electrons and muons (Section 4.6) are directly affected by the accuracy of the jet energy measurement. If the additional energy from jets entering the lepton cone is underestimated, so will be the isolation. The same correction factors used above for jet ID are therefore applied to adjust the calorimeter isolation cuts.

The recalculated and original acceptances are given in Table 7.4. The variation is on the order of $2 \%$ and well within the statistical error. This is consistent with the top dilepton value, so we assign a $2 \%$ uncertainty for the jet energy scale systematic. 


\subsection{Luminosity}

The number of events we expect from signal is determined by the cross section for gluino pair production and the total integrated luminosity collected for the Run I dataset. Any uncertainty in the luminosity directly impacts the number of events we expect to observe in the data, not the acceptance rate, and should be folded into the appropriate systematic error.

The luminosity is measured using scintillation counters which are placed upstream and downstream of the interaction region and which provided a "minimum-bias" trigger $[17,54]$. For Run I, two sets of 16 scintillator counters were located at $\pm 5.82 \mathrm{~m}$ and were arranged in a rectangle about the beampipe. This provided polar angular coverage of $0.32^{\circ}$ to $4.47^{\circ}$, or 3.24 to 5.90 in $\eta$. These beam-beam counters (BBC) measured intime and out of time hits, with respect to a 30 ns window about the expected arrival time of the colliding beams.

A coincidence of an intime hit in the east side counters with an intime hit on the west side is registered as a BBC interaction. Given the cross section for events registering in the beam-beam counters, $\sigma_{B B C}$, the instantaneous and total integrated luminosities can be calculated from the rate $\left(R_{B B C}\right)$ and total number $\left(N_{B B C}\right)$ of BBC coincidences using:

$$
\mathcal{L}=\frac{R_{B B C}}{\sigma_{B B C}}, \quad L_{\text {total }}=\frac{N_{B B C}}{\sigma_{B B C}}
$$

As the cross section is energy-dependent, dedicated runs were performed to directly measure the elastic and total cross sections at $\sqrt{s}=1.8 \mathrm{TeV} . \sigma_{B B C}(1800)$ was measured to be $(51.15 \pm 1.60 \mathrm{mb})$ [55], and the $3.1 \%$ uncertainty is the largest contribution to the luminosity systematic error.

A comprehensive study of the luminosity measurement has been performed by CDF [56]. The Run Ia integrated luminosity was calculated to be $19.7 p b^{-1}$, with a $3.6 \%$ error. Additional contributions to the uncertainty come from attempts to estimate the multiple interaction rate, usage of the average luminosity and corrections applied to account for variation in the beam intensity within each run, and interactions in the beam halo. 
In Run Ib, the instantaneous luminosity was measured frequently, up to 500 times over ten hours, during which the intensity may drop by a factor of two. The total luminosity was measured to be $86.5 \mathrm{pb}^{-1}$ with a $4.1 \%$ error. A cross check of the usage of instantaneous rather than average luminosity was performed using the ratio of $W \rightarrow e \nu$ events observed in Run Ib versus Ia. The expected luminosity value from $W$ events is $2.3 \%$ lower than the measured value, so this discrepancy is folded into the systematic error.

For each analysis, the error on the overall Run I luminosity is dependent upon the fraction of data taken from each dataset. For the $W \rightarrow e \nu$ analysis, the total luminosity is $(106.1 \pm 4.1) p b^{-1}$. The $3.9 \%$ uncertainty is considered applicable to most analyses, so we use this value for our systematic error.

\subsection{Remaining sources of error}

The remaining sources of systematic uncertainty are concerned with the detector or event selection procedure and are not specific to the particular process we are simulating. Conservative estimates were made for the top dilepton analysis and, even so, are typically small.

\subsubsection{Photon conversion removal}

The photon conversion removal discussed in Section 4.1.3 tends to be overefficient and removes a limited fraction of events from the sample by misidentifying electrons from signal as conversions. In order to estimate the effect of the algorithm on the top dilepton analysis, two $t \bar{t}$ samples were processed with and without the routine enabled. The decrease in acceptance due to the algorithm was used to calculate the systematic error.

An 80,000 event top sample [57] was created using PYTHIA version 5.6 and the CTEQ 2L PDF, with a generated top mass of $175 \mathrm{GeV}$. The dilepton channel acceptance, $\epsilon_{\text {dil }}$ decreased by $(4.0 \pm 0.7) \%$ with the inclusion of the conversion removal routine, with $(7.0 \pm 2.5) \%$ decrease in the ee mode and $(4.7 \pm 1.1) \%$ in the $e \mu$ mode. 


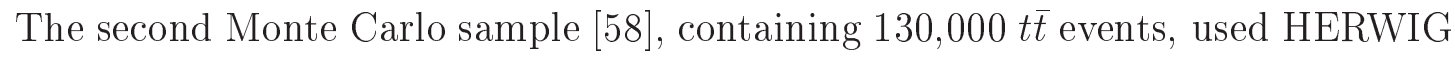
and the MRSD0' distribution function. In this case, the top dilepton acceptance was reduced by $(1.8 \pm 0.4) \%$ : $(2.9 \pm 1.3) \%$ for dilectrons and $(2.2 \pm 0.6) \%$ for $e \mu$ events.

Even at the lepton ID stage, the decrease in acceptance was $(5.6 \pm 0.4) \%$ and $(4.0 \pm 0.7) \%$ for PYTHIA and HERWIG, respectively. There exists some difference between the two generators, although this was not explored in further detail. A systematic error of $2 \%$ was assigned for the uncertainty in the effect of including the photon conversion removal. This systematic is directly relevant to the like-sign top dilepton channel for the gluino analysis, so we adopt the $2 \%$ uncertainty.

\subsubsection{Lepton ID efficiency}

The largest systematic error for the top dilepton signature is due to the lepton ID efficiency. As seen in Figure 6.7 and Table 6.7, there are significant discrepancies between the lepton ID efficiencies for $Z^{0} \rightarrow l l$ data and Monte Carlo. There is some uncertainty as to how accurate the scale factors calculated from $Z^{0}$ studies are

for $t \bar{t}$ and $\tilde{g} \rightarrow t \overline{\tilde{t}}$ events, considering the different event topologies and isolation distributions.

To determine the uncertainty for $t \bar{t}$, the dilepton acceptance obtained from Monte Carlo was recalculated. The procedure used new scale factors where the $Z^{0}$ data efficiency was increased and decreased by half the difference between the data and Monte Carlo efficiencies. This resulted in a variation of $\pm 7 \%$ in the acceptance, which is approximately half of the $13 \%$ reduction in acceptance that comes from applying the scale factors to $t \bar{t}$ Monte Carlo. While the effect of the lepton ID correction on $\tilde{g} \tilde{g}$ samples is in the $14-17 \%$ range, likely due to a higher proportion of events with isolation values in the highest range, we employ the $7 \%$ figure systematic error from top dilepton events.

\subsubsection{Lepton isolation}

The track and calorimeter isolation requirement, discussed in Section 4.6, depends on accurate reproduction of lepton isolation in Monte Carlo. As described in Sec- 
tion 4.6, the isolation requirement is very efficient for leptons from $W$ and $\tau$ decays and is designed to remove events where the lepton originates from a $b$ decay. For top events, the cut efficiency is $95 \%$, where the $5 \%$ loss is dominated by semileptonic $b$ decays.

The systematic uncertainty due to the isolation cut was calculated using the HERWIG $t \bar{t}$ sample described above. The method used was thus: one presumes that the Monte Carlo underestimates the isolation by a factor of 2, so the track and calorimeter cuts are set to 0.05 and the dilepton channel acceptance is recalculated for the HERWIG sample. The acceptance dropped by $2 \%$, from $(.784 \pm .025) \%$ to $(.769 \pm .024) \%$. This percentage is adopted as the systematic error due to simulation of the isolation.

\subsubsection{Tracking efficiency}

During Run Ib, the tracking ability of the CTC was diminished slightly as the system degraded. Low momentum tracks were expected to be the most affected, while the efficiency of identifying high- $p_{T}$ tracks, such as those for the top dilepton signature, should be unvaried. A limited number of studies have been done to understand this effect, and an estimate of $2 \%$ systematic uncertainty is ascribed to the tracking efficiency.

\subsubsection{Trigger efficiency}

In Section 3.8, the effect of the central electron and muon triggers on the event acceptance is discussed. The central electron trigger is nearly fully efficient, while the muon trigger lowers the top dilepton acceptance by approximately $2 \%$. The relatively minor impact of the triggers implies that any uncertainty in the trigger simulation should be minuscule. A conservative estimate of $1 \%$ is assigned to the trigger efficiency. 


\subsection{Total systematic uncertainty}

In order to calculate the overall systematic uncertainty on the event acceptance, all systematic errors are added in quadrature. Although some sources of systematic error are correlated, the assumption that they are not provides an overestimate of the total uncertainty. This conservative approach does not weigh as heavily upon the limit calculation in Chapter 9 as does the number of observed events compared to the expected background.

Strictly speaking, the uncertainty on the total integrated luminosity of our Run I dataset bears directly on the number of expected events and is independent of the acceptance rate. For simplicity we shall fold the luminosity uncertainty into the error on the acceptance. All errors are summarized in Table 7.5, and the total systematic uncertainty is $10 \%$. 


\begin{tabular}{|c|c|}
\hline Source & $\%$ Error \\
\hline Lepton ID efficiencies & $7 \%$ \\
\hline Luminosity & $4 \%$ \\
\hline Parton distribution function & $3 \%$ \\
\hline IS and FS gluon radiation & $3 \%$ \\
\hline$Q^{2}$ scale & $2 \%$ \\
\hline Jet energy scale & $2 \%$ \\
\hline Conversion removal & $2 \%$ \\
\hline Isolation efficiency & $2 \%$ \\
\hline Tracking efficiency & $2 \%$ \\
\hline Trigger efficiency & $1 \%$ \\
\hline Total & $10 \%$ \\
\hline
\end{tabular}

Table 7.5: Summary of all sources of systematic error. The overall systematic uncertainty on the dilepton acceptance is obtained by adding all errors in quadrature. 


\section{Chapter 8}

\section{Background Estimate}

The Lepton ID requirements described in Chapter 4 are designed to reduce the contribution of background processes and emphasize the signal. However, it is neither possible nor always desirable to completely exclude the background from passing the kinematic cuts. Therefore, in this chapter we examine the possible sources of background and determine their contribution to the number of Run I events which have passed the analysis.

When the top dilepton analysis was originally performed, the background sources with the largest contributions were identified as:

- Drell-Yan: dilepton production from $\gamma$ or $Z^{0}$

- $Z^{0} \longrightarrow \tau^{+} \tau^{-}: Z^{0}$ production and leptonic tau decay

- Dibosons: $W W, W Z, Z Z \longrightarrow l^{+} l^{-}+X$

- Fake leptons: mismeasured jets which pass the lepton ID requirements

- $b \bar{b}, c \bar{c}$ : semileptonic decay of both light quarks

- Radiative $Z^{0}$ decays: additional leptons from Drell-Yan of a radiated photon

None of these processes is expected to directly mimic all aspects of this particular signal for top: 2 high $p_{T}$ leptons, 2 or more jets, and a large transverse energy imbalance. Rather, the more likely scenario is that each background yields some 
portion of the signal. For instance, a background event may contain two oppositesign leptons yet lack sufficient jet activity or missing transverse energy. The remaining event characteristics must therefore arise from mismeasurement (which may create $\mathscr{E}_{T}$, or extra leptons from jet fakes) and/or underlying event processes (to provide any otherwise missing jet production). These factors offer a tiny but finite likelihood that a background process may mimic the $t \bar{t}$ signature.

For the same-sign gluino search, the situation changes somewhat. Most of the top dilepton backgrounds rely on either the direct creation of an opposite-sign lepton pair or the decay of oppositely signed parent particles into daughter leptons, both of which naturally follow from conservation of charge. No single isolated Standard Model reaction, such as Drell-Yan, can directly produce a like-sign pair of leptons in the absence of an initial state with charge \pm 2 . Therefore, in order for this type of background process to contribute to the analysis, the charge of one of lepton must be mismeasured such that an opposite-sign pair appears to be same-sign. The probability of mismeasurement is small, which pushes the likelihood of these backgrounds contributing to the observed signal even smaller. Other backgrounds, such as $W Z$ and $Z Z$, contribute through final states comprised of multiple leptons, though the branching ratios for such modes are substantially smaller than their hadronic counterparts.

Top production also functions as a background to gluino production. This occurs primarily through the dilepton channel, although the semileptonic and, to a much lesser extent, all-hadronic channels also contribute. The dilepton mode depends almost exclusively upon $W^{ \pm}$decay for high- $p_{T}$ lepton production, and in the simplest scenario, the sign of one lepton is incorrectly measured. However, the semileptonic decay of the $b$ quark $\left(b \rightarrow c l \nu_{l}\right.$ or $\left.b \rightarrow c \rightarrow s l \nu_{l}\right)$ affords an alternate source of leptons. The smaller branching ratio and much softer momentum distribution limit the signal enhancement, but the presence of trilepton events in the top sample indicate the potential for this mode to contribute.

The most significant backgrounds are expected to be $t \bar{t}$ and fake leptons, with minor contributions from $b \bar{b}, c \bar{c}$, dibosons, and Drell-Yan. In the following sections we shall discuss each of these background sources and quantify their expected contri- 
butions to the same-sign dilepton signal.

\subsection{Background samples}

To estimate the contribution of each background to the observed number of events, we generate Monte Carlo samples for each process and analyze them using the same code used to process the data and signal Monte Carlo. Just as for signal Monte Carlo, one should at least generate samples which correspond to a total integrated luminosity comparable to that of Run I. For backgrounds where the expected contribution is of order 1 or smaller, the statistical errors are of the same magnitude or greater. Thus it becomes necessary to create much larger samples to reduce the error and gain confidence in the resulting estimate.

Generation of large background MC samples is a long, time-consuming process, and sometimes involves fine tuning of the $\mathrm{MC}$ generator to accurately model the physics. Fortunately, these background processes are often common to several analyses. These factors encourage the formation of standardized samples that are utilized by all involved in a particular analysis group.

The Supersymmetry analysis subgroup at CDF has created an extensive archive of MC samples for use by its members. Many of these samples include upwards of several hundred thousand events and, depending on the process and event filter used, correspond to total integrated luminosities ranging from a few hundred $p b^{-1}$ to a few $f b^{-1}$, which translates into $3-60$ times the amount of data collected during Run I. For the simulation of some processes, minimal cuts are placed at the generator stage (before detector simulation) to safely eliminate those events which could not have passed the initial selection criteria for any formal analyses. This reduces the samples to a more manageable size. Any generator-level cuts will be described in each section below.

Two versions of the ISAJET Monte Carlo generator were used to produce the samples: v7_06 and v7_20. In turn, these were paired with a variety of parton distribution functions. CTEQ-2L, MRSD0', and GRV-LO were used with ISAJET v7_06, while the more recent parton descriptions CTEQ-3L and GRV-94 LO were used with 
ISAJET v7_20. Our analysis is relatively insensitive to the choice of PDF and generator (as described in Section 7.1) due to the relatively tight selection cuts employed. Because the uncertainty in the background estimate is statistics limited, multiple samples are combined for each individual process, regardless of the PDF or Monte Carlo version used to generate the sample, to improve statistical errors.

As was the case with simulation of $\tilde{g} \tilde{g}$ events, the results of applying the top dilepton analysis to background Monte Carlo must be corrected to account for the discrepancy in lepton ID efficiencies between MC and data. The same procedure used in Section 6.4 is applied to any background events which pass all of the selection criteria. We refer the reader back to Table 6.7 and Figure 6.7 for further details.

\section{2 $b \bar{b}$ and $c \bar{c}$ production}

In the Standard Model $t \bar{t}$ dilepton channel, bottom and charm quark pair production offer the smallest background contribution. For the CDF top quark discovery paper [59], the estimated background from $b \bar{b}$ was $0.03 \pm 0.02$ events in $67 p b^{-1}$ [60]. For the Run I estimate, this result was simply scaled to the appropriate luminosity for the top sample $\left(109 p b^{-1}\right)$ to arrive at $0.05 \pm 0.03$ events.

The $p_{T}$ spectrum for leptons originating from $b$ decays in $b \bar{b}$ production events falls off rapidly [61], and the probability for a $b$ quark to produce a lepton with $p_{T}>20$ $\mathrm{GeV}$ is on the order of $10^{-4}$. A light flavor quark must be highly energetic in order to produce a high- $p_{T}$ lepton. The decay products of an energetic light quark receive a boost in the laboratory frame and tend to be highly collimated, in contrast to particles created by the decay of a massive top quark. A lepton produced by $b$ or $c$ quark decay is immersed within the jet, which greatly increases the likelihood that multiple tracks from the surrounding hadronic activity will fall into the angular cone surrounding the lepton. Even if the lepton manages to pass the isolation requirement (or if the second lepton satisfies the isolation cut), measurements of the lepton kinematic variables become "contaminated," lowering the ID efficiency.

The dependence of lepton quality upon the source is well illustrated in Figure 8.1. Here we compare the lepton ID efficiency for Tight Central Electrons (TCE) originat- 


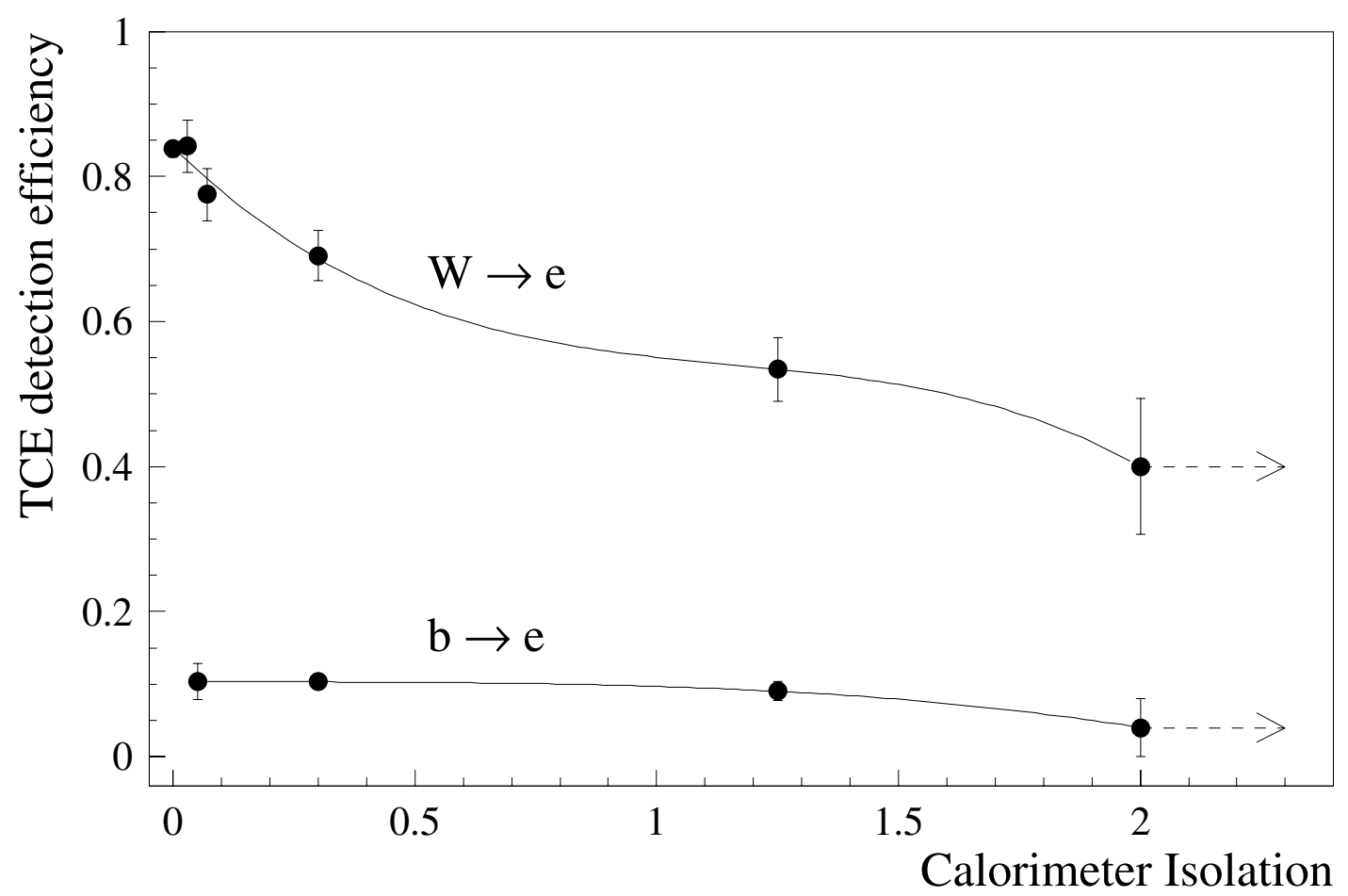

Figure 8.1: Detection efficiency for TCE category electrons produced by the decay of $W$ 's and $b$ quarks in $t \bar{t}$ Monte Carlo events, as a function of lepton isolation.

ing from $W$ and $b$ decays in $t \bar{t}$ Monte Carlo events. The results are equally applicable to bottom quarks in $b \bar{b}$ events. Across the spectrum of calorimeter isolation, the efficiency is significantly lower for electrons from $b$ decay.

Event acceptance is poor due to the low lepton ID efficiency and the isolation requirement. In addition, $b \bar{b}, c \bar{c}$ events where both quarks decay leptonically $\left(q \rightarrow l \nu_{l} q^{\prime}\right)$, are unlikely to satisfy other requirements placed on the event. The two neutrinos tend to lie back-to-back and negate any contribution to the missing energy $\left(\mathbb{E}_{T}\right)$ measurement. Without higher order QCD processes, these events also lack sufficient jet activity to pass the 2-jet requirement.

Conversely, one advantage afforded to light quark pair production is the capability 
of directly producing a like-sign dilepton signal, independent of charge mismeasurement or other detector effects. The $b$ quark lifetime is sufficiently long for the quark to hadronize into a $B$ meson $\left(B^{0}\right.$ or $\left.B^{ \pm}\right)$. $B^{0} \bar{B}^{0}$ mixing allows the $b$ quark on one side of a $b \bar{b}$ event to oscillate into its charge conjugate state, and subsequent leptonic decays of both $b$ quarks will produce same-sign leptons. Alternately, the cascade decay $b \rightarrow c \rightarrow s l \nu_{l}$ creates a lepton with the opposite charge from one produced by ordinary semileptonic $b$ decay. The combination of these two effects causes about $35 \%$ of dilepton events from $b \bar{b}$ to be like-sign.

ISAJET does not properly generate like-sign dileptons due to $B^{0} \bar{B}^{0}$ mixing. Therefore, corrections accounting for the mixing effect must be inserted by hand. The proper number of opposite-sign and like-sign events is calculated from the Monte Carlo results using [63]:

$$
\begin{aligned}
& N_{O S}=\left((1-\chi)^{2}+\chi^{2}\right) O S_{I S A J E T}+2 \chi(1-\chi) L S_{I S A J E T} \\
& N_{L S}=2 \chi(1-\chi) L S_{I S A J E T}+\left((1-\chi)^{2}+\chi^{2}\right) L S_{I S A J E T}
\end{aligned}
$$

where $\chi$ is the average mixing parameter. Using the CDF measurement of $\chi=$ $0.118 \pm 0.008 \pm 0.020[63]$, this becomes:

$$
\begin{aligned}
& N_{O S}=(0.792 \pm 0.044) O S_{I S A J E T}+(0.208 \pm 0.044) L S_{I S A J E T} \\
& N_{L S}=(0.208 \pm 0.044) O S_{I S A J E T}+(0.792 \pm 0.044) L S_{I S A J E T}
\end{aligned}
$$

The low probability for a $b \bar{b}$ or $c \bar{c}$ event to pass the lepton identification stage and subsequent dilepton selection cuts is compensated for by the large cross sections compared to $t \bar{t}$ production: $\sigma_{b \bar{b}}$ is approximately $5 \times 10^{6}$ larger than $\sigma_{t \bar{t}}$. This translates into large numbers of events even for luminosities on the order of Run I. Monte Carlo simulation of $b \bar{b}, c \bar{c}$ is extremely time-consuming and requires an enormous amount of disk space. Therefore, the following cuts were applied at the generator level for the $b \bar{b}$ and $c \bar{c}$ samples. At least one $b$ or $c$ quark was required to have $p_{T}>10 \mathrm{GeV}$ and pseudorapidity $|\eta|<4$.0. Each event also needed to have a least one central lepton (electron or muon) with $\left(p_{T}>9.0 \mathrm{GeV}\right.$ and $\left.|\eta|<1.5\right)$ or two softer leptons with $\left(p_{T}>2.8 \mathrm{GeV}\right.$ and $\left.|\eta|<3.0\right)$. These cuts provide full efficiency for central leptons with $p_{T}>10 \mathrm{GeV}$ and $>3 \mathrm{GeV}$, respectively [62]. 
To further reduce the datasets to a more manageable size, the Monte Carlo simulations are divided into smaller subsets. Samples were created for the three main production modes of $b \bar{b}$ and $c \bar{c}$ : direct production $(q \bar{q}, g g \rightarrow b \bar{b}, c \bar{c})$; initial state gluon radiation, where the radiated gluon splits into $q \bar{q}$; and final state gluon splitting into $q \bar{q}$. Moreover, each production mode was subsequently divided into three momentum ranges, according to the highest $p_{T}$ quark in the event: $10 \leq p_{T} \leq 25 \mathrm{GeV}$, $25 \leq p_{T} \leq 50 \mathrm{GeV}$, and $50 \leq p_{T} \leq 500 \mathrm{GeV}$.

The results of the like-sign dilepton analysis applied to the nine subsamples are provided in Tables 8.1 (direct production), 8.2 (IS gluon radiation), and 8.3 (FS gluon radiation). In addition, we must determine the acceptance rate for opposite-sign events in order to compensate for the absence of $B^{0} \bar{B}^{0}$ mixing effects in ISAJET. The opposite-sign analysis of the $b \bar{b}, c \bar{c}$ samples is presented in Tables 8.4 (direct production), 8.5 (IS gluon radiation), and 8.6 (FS gluon radiation).

In each subsample, independent of the source of quark pair production or parton momentum range, zero events pass the analysis. In many cases, no events manage to satisfy even the basic requirement of the presence of two high- $p_{T}$ leptons. Once it became apparent that even the highest quark momentum samples failed to produce events capable of meeting the initial few selection criteria, further study of the lowest momentum range samples was abandoned.

Out of over 1.6 million prefiltered events processed, a total of 110 pass the lepton ID stage and cosmic ray filter. Three direct production events (of 84) contain same-sign dileptons. Of these, only one passes the isolation and $Z$ mass filters but subsequently fails the $\mathbb{E}_{T}$ cut. No IS gluon radiation events (of 10) contain same-sign dileptons, and only 2 of 15 FS gluon events do. Both of these fail the isolation cut. The opposite-sign events fare no better, with only two direct production events in the middle momentum range passing the isolation stage and $Z$ filter, only to fail the $\mathbb{E}_{T}$ cut.

Because no MC events passed the analysis, the expected number of same- and opposite-sign dilepton events from $b \bar{b}, c \bar{c}$ is $N_{b g}^{b \bar{b}, c \bar{c}}=0.0$, and no correction is necessary to account for the missing $B^{0} \bar{B}^{0}$ mixing effects in ISAJET. Because we cannot be completely certain that additional Monte Carlo samples would not have yielded a 


\begin{tabular}{|c|c|c|c|c|c|c|c|c|c|}
\hline & & & \multicolumn{6}{|c|}{ DILEPTON CUT } & \multirow{3}{*}{$\begin{array}{l}\text { Expected \# } \\
\text { Scaled by } \\
\text { Lep ID eff }\end{array}$} \\
\hline Bkgd & No. of & Lum & Lepton & Cosmic & Same & & & $N_{j}>10 \mathrm{GeV}$ & \\
\hline Sample & Events & $p b^{-1}$ & ID & Ray & Sign & Isolation $M_{l l}$ & $\mathbb{E}_{T} \quad \Delta \phi\left(\mathbb{E}_{T}, l\right) \quad \Delta \phi\left(\mathbb{E}_{T}, j\right)$ & $\begin{array}{lll}0 & 1 & \geq 2\end{array}$ & \\
\hline
\end{tabular}

$b \bar{b}, c \bar{c}: 10.0 \leq p_{T} \leq 25.0$, direct production

\begin{tabular}{|l|l|l|lllll|lllll|llll|l|}
\hline CTEQ2L & 100000 & 62.7 & 0 & 0 & 0 & 0 & 0 & 0 & 0 & 0 & 0 & 0 & 0 & 0.0 \\
\hline \hline
\end{tabular}

$b \bar{b}, c \bar{c}: 25.0 \leq p_{T} \leq 50.0$, direct production

\begin{tabular}{|c|c|c|cccccc|ccc|ccc|c|}
\hline CTEQ3L & 100000 & 85.9 & 9 & 9 & 0 & 0 & 0 & 0 & 0 & 0 & 0 & 0 & 0 & 0.0 \\
GRV94LO & 95209 & 89.9 & 8 & 8 & 1 & 1 & 1 & 0 & 0 & 0 & 0 & 0 & 0 & 0.0 \\
MRSD0, & 99997 & 76.0 & 12 & 12 & 1 & 0 & 0 & 0 & 0 & 0 & 0 & 0 & 0 & 0.0 \\
CTEQ2L & 100000 & 88.1 & 4 & 4 & 0 & 0 & 0 & 0 & 0 & 0 & 0 & 0 & 0 & 0.0 \\
GRV-LO & 100000 & 98.3 & 7 & 7 & 0 & 0 & 0 & 0 & 0 & 0 & 0 & 0 & 0 & 0.0 \\
\hline Total & 495206 & 438.2 & 40 & 40 & 2 & 1 & 1 & 0 & 0 & 0 & 0 & 0 & 0 & 0.0 \\
\hline \hline
\end{tabular}

$b \bar{b}, c \bar{c}: 50.0 \leq p_{T} \leq 500.0$, direct production

\begin{tabular}{|c|c|c|ccccc|cccc|cc|c|}
\hline CTEQ3L & 50000 & 282.1 & 24 & 24 & 3 & 0 & 0 & 0 & 0 & 0 & 0 & 0 & 0. & 0.0 \\
GRV-LO & 50000 & 336.7 & 20 & 20 & 0 & 0 & 0 & 0 & 0 & 0 & 0 & 0 & 0. & 0.0 \\
\hline Total & 100000 & 618.8 & 44 & 44 & 3 & 0 & 0 & 0 & 0 & 0 & 0 & 0 & 0. & 0.0 \\
\hline \hline
\end{tabular}

Table 8.1: Like-sign analysis of quark pair production $(b \bar{b}, c \bar{c})$ samples which include only direct production. 


\begin{tabular}{|c|c|c|c|c|c|c|c|c|c|c|}
\hline \multirow{3}{*}{$\begin{array}{l}\text { Bkgd } \\
\text { Sample }\end{array}$} & \multirow{3}{*}{$\begin{array}{l}\text { No. of } \\
\text { Events }\end{array}$} & \multirow{3}{*}{$\begin{array}{l}\text { Lum } \\
p b^{-1}\end{array}$} & \multicolumn{7}{|c|}{ DILEPTON CUT } & \multirow{3}{*}{$\begin{array}{l}\text { Expected \# } \\
\text { Scaled by } \\
\text { Lep ID eff }\end{array}$} \\
\hline & & & Lepton & Cosmic & Same & & & & $N_{j}>10 \mathrm{GeV}$ & \\
\hline & & & ID & Ray & Sign & Isolation $M_{l l}$ & $\mathbb{E}_{T} \quad \Delta \phi\left(\mathbb{E}_{T}, l\right)$ & $\Delta \phi\left(\mathbb{E}_{T}, j\right)$ & $\begin{array}{lll}0 & 1 & \geq 2\end{array}$ & \\
\hline
\end{tabular}

$b \bar{b}, c \bar{c}: 10.0 \leq p_{T} \leq 25.0$, initial state gluon radiation

\begin{tabular}{|l|l|l|lllll|llll|llll|l|}
\hline CTEQ2L & 100000 & 133.5 & 0 & 0 & 0 & 0 & 0 & 0 & 0 & 0 & 0 & 0 & 0 & 0.0 \\
\hline \hline
\end{tabular}

$b \bar{b}, c \bar{c}: 25.0 \leq p_{T} \leq 50.0$, initial state gluon radiation

\begin{tabular}{|c|c|c|ccccc|ccc|ccc|c|}
\hline CTEQ3L & 100000 & 145.6 & 1 & 1 & 0 & 0 & 0 & 0 & 0 & 0 & 0 & 0 & 0 & 0.0 \\
MRSD0 & 100000 & 157.3 & 0 & 0 & 0 & 0 & 0 & 0 & 0 & 0 & 0 & 0 & 0 & 0.0 \\
GRV-LO & 100000 & 145.4 & 0 & 0 & 0 & 0 & 0 & 0 & 0 & 0 & 0 & 0 & 0 & 0.0 \\
\hline Total & 300000 & 448.3 & 1 & 1 & 0 & 0 & 0 & 0 & 0 & 0 & 0 & 0 & 0 & 0.0 \\
\hline \hline
\end{tabular}

$b \bar{b}, c \bar{c}: 50.0 \leq p_{T} \leq 500.0$, initial state gluon radiation

\begin{tabular}{|l|l|l|lllll|llll|lll|l|}
\hline CTEQ3L & 92781 & 721.2 & 10 & 10 & 0 & 0 & 0 & 0 & 0 & 0 & 0 & 0 & 0 & 0.0 \\
\hline \hline
\end{tabular}

Table 8.2: Like-sign analysis of quark pair production $(b \bar{b}, c \bar{c})$ samples which involve $q \bar{q}$ creation from initial state gluon radiation. 


\begin{tabular}{|c|c|c|c|c|c|c|c|c|c|c|}
\hline \multirow{3}{*}{$\begin{array}{c}\text { Bkgd } \\
\text { Sample }\end{array}$} & \multirow{3}{*}{$\begin{array}{l}\text { No. of } \\
\text { Events }\end{array}$} & \multirow{3}{*}{$\begin{array}{l}\text { Lum } \\
p b^{-1}\end{array}$} & \multicolumn{7}{|c|}{ DILEPTON CUT } & \multirow{3}{*}{$\begin{array}{l}\text { Expected \# } \\
\text { Scaled by } \\
\text { Lep ID eff }\end{array}$} \\
\hline & & & Lepton & Cosmic & Same & & & & $N_{j}>10 \mathrm{GeV}$ & \\
\hline & & & ID & Ray & Sign & Isolation $M_{l l}$ & $E_{T} \quad \Delta \phi\left(E_{T}, l\right)$ & $\Delta \phi\left(\mathbb{E}_{T}, j\right)$ & $0 \begin{array}{lll}0 & 1 & \geq 2\end{array}$ & \\
\hline
\end{tabular}

$b \bar{b}, c \bar{c}: 10.0 \leq p_{T} \leq 25.0$, final state gluon radiation

\begin{tabular}{|l|l|l|lllll|llll|lll|l|l|}
\hline CTEQ2L & 25000 & 104.3 & 0 & 0 & 0 & 0 & 0 & 0 & 0 & 0 & 0 & 0 & 0 & 0.0 \\
\hline \hline
\end{tabular}

$b \bar{b}, c \bar{c}: 25.0 \leq p_{T} \leq 50.0$, final state gluon radiation

\begin{tabular}{|c|c|c|ccccc|cccc|ccc|c|}
\hline CTEQ3L & 100000 & 167.4 & 0 & 0 & 0 & 0 & 0 & 0 & 0 & 0 & 0 & 0 & 0 & 0.0 \\
GRV94LO & 100000 & 192.8 & 0 & 0 & 0 & 0 & 0 & 0 & 0 & 0 & 0 & 0 & 0 & 0.0 \\
\hline Total & 200000 & 360.2 & 0 & 0 & 0 & 0 & 0 & 0 & 0 & 0 & 0 & 0 & 0 & 0.0 \\
\hline \hline
\end{tabular}

$\bar{b}, c \bar{c}: 50.0 \leq p_{T} \leq 500.0$, final state gluon radiation

\begin{tabular}{|c|c|c|ccccc|cccc|cc|c|}
\hline CTEQ3L & 100000 & 321.3 & 7 & 7 & 0 & 0 & 0 & 0 & 0 & 0 & 0 & 0 & 0 & 0.0 \\
GRV94LO & 100000 & 389.9 & 8 & 8 & 2 & 0 & 0 & 0 & 0 & 0 & 0 & 0 & 0 & 0.0 \\
\hline Total & 200000 & 711.2 & 15 & 15 & 2 & 0 & 0 & 0 & 0 & 0 & 0 & 0 & 0 & 0.0 \\
\hline \hline
\end{tabular}

Table 8.3: Like-sign analysis of quark pair production $(b \bar{b}, c \bar{c})$ samples which involve $q \bar{q}$ creation from final state gluon radiation. 


\begin{tabular}{|c|c|c|c|c|c|c|c|c|}
\hline & & & \multicolumn{5}{|c|}{ DILEPTON CUT } & \multirow{3}{*}{$\begin{array}{l}\text { Expected \# } \\
\text { Scaled by } \\
\text { Lep ID eff }\end{array}$} \\
\hline Bkgd & No. of & Lum & Lepton & Cosmic & Opp. & & $N_{j}>10 \mathrm{GeV}$ & \\
\hline Sample & Events & $p b^{-1}$ & ID & Ray & Sign Isolation $M_{l l}$ & $\mathbb{E}_{T} \quad \Delta \phi\left(\mathbb{E}_{T}, l\right) \quad \Delta \phi\left(\mathbb{E}_{T}, j\right)$ & $\begin{array}{lll}0 & 1 & \geq 2\end{array}$ & \\
\hline
\end{tabular}

$\bar{b}, c \bar{c}: 10.0 \leq p_{T} \leq 25.0$, direct production

\begin{tabular}{|l|l|l|lllll|llll|llll|l|}
\hline CTEQ2L & 100000 & 62.7 & 0 & 0 & 0 & 0 & 0 & 0 & 0 & 0 & 0 & 0 & 0 & 0.0 \\
\hline \hline
\end{tabular}

$b \bar{b}, c \bar{c}: 25.0 \leq p_{T} \leq 50.0$, direct production

\begin{tabular}{|c|c|c|ccccc|ccc|ccc|c|}
\hline CTEQ3L & 100000 & 85.9 & 9 & 9 & 9 & 1 & 1 & 0 & 0 & 0 & 0 & 0 & 0 & 0.0 \\
GRV94LO & 95209 & 89.9 & 8 & 8 & 7 & 0 & 0 & 0 & 0 & 0 & 0 & 0 & 0 & 0.0 \\
MRSD0, & 99997 & 76.0 & 12 & 12 & 11 & 0 & 0 & 0 & 0 & 0 & 0 & 0 & 0 & 0.0 \\
CTEQ2L & 100000 & 88.1 & 4 & 4 & 4 & 1 & 1 & 0 & 0 & 0 & 0 & 0 & 0 & 0.0 \\
GRV-LO & 100000 & 98.3 & 7 & 7 & 7 & 0 & 0 & 0 & 0 & 0 & 0 & 0 & 0 & 0.0 \\
\hline Total & 495206 & 438.2 & 40 & 40 & 38 & 2 & 2 & 0 & 0 & 0 & 0 & 0 & 0 & 0.0 \\
\hline \hline
\end{tabular}

$b \bar{b}, c \bar{c}: 50.0 \leq p_{T} \leq 500.0$, direct production

\begin{tabular}{|c|c|c|ccccc|cccc|ccc|c|}
\hline CTEQ.3L & 50000 & 282.1 & 24 & 24 & 21 & 0 & 0 & 0 & 0 & 0 & 0 & 0 & 0. & 0.0 \\
GRV-LO & 50000 & 336.7 & 20 & 20 & 20 & 0 & 0 & 0 & 0 & 0 & 0 & 0 & 0. & 0.0 \\
\hline Total & 100000 & 618.8 & 44 & 44 & 41 & 0 & 0 & 0 & 0 & 0 & 0 & 0 & 0. & 0.0 \\
\hline \hline
\end{tabular}

Table 8.4: Opposite-sign analysis of quark pair production $(b \bar{b}, c \bar{c})$ samples which include only direct production. 


\begin{tabular}{|c|c|c|c|c|c|c|c|c|c|c|}
\hline \multirow{3}{*}{$\begin{array}{c}\text { Bkgd } \\
\text { Sample }\end{array}$} & \multirow{3}{*}{$\begin{array}{l}\text { No. of } \\
\text { Events }\end{array}$} & \multirow{3}{*}{$\begin{array}{l}\text { Lum } \\
p b^{-1}\end{array}$} & \multicolumn{7}{|c|}{ DILEPTON CUT } & \multirow{3}{*}{$\begin{array}{l}\text { Expected \# } \\
\text { Scaled by } \\
\text { Lep ID eff }\end{array}$} \\
\hline & & & Lepton & Cosmic & Opp. & & & & $N_{j}>10 \mathrm{GeV}$ & \\
\hline & & & ID & Ray & Sign & Isolation $M_{l l}$ & $\mathbb{E}_{T} \quad \Delta \phi\left(\mathbb{E}_{T}, l\right)$ & $\Delta \phi\left(\hat{E}_{T}, j\right)$ & $\begin{array}{lll}0 & 1 & \geq 2\end{array}$ & \\
\hline
\end{tabular}

$b \bar{b}, c \bar{c}: 10.0 \leq p_{T} \leq 25.0$, initial state gluon radiation

\begin{tabular}{|l|l|l|lllll|llll|lll|l|}
\hline CTEQ2L & 100000 & 133.5 & 0 & 0 & 0 & 0 & 0 & 0 & 0 & 0 & 0 & 0 & 0 & 0.0 \\
\hline \hline
\end{tabular}

$b \bar{b}, c \bar{c}: 25.0 \leq p_{T} \leq 50.0$, initial state gluon radiation

\begin{tabular}{|c|c|c|ccccc|cccc|ccc|c|}
\hline CTEQ3L & 100000 & 145.6 & 1 & 1 & 1 & 0 & 0 & 0 & 0 & 0 & 0 & 0 & 0 & 0.0 \\
MRSD0' & 100000 & 157.3 & 0 & 0 & 0 & 0 & 0 & 0 & 0 & 0 & 0 & 0 & 0 & 0.0 \\
GRV-LO & 100000 & 145.4 & 0 & 0 & 0 & 0 & 0 & 0 & 0 & 0 & 0 & 0 & 0 & 0.0 \\
\hline Total & 300000 & 448.3 & 1 & 1 & 1 & 0 & 0 & 0 & 0 & 0 & 0 & 0 & 0 & 0.0 \\
\hline \hline
\end{tabular}

$b \bar{b}, c \bar{c}: 50.0 \leq p_{T} \leq 500.0$, initial state gluon radiation

\begin{tabular}{|l|l|l|lllll|lllll|lllll|}
\hline CTEQ3L & 92781 & 721.2 & 10 & 10 & 10 & 0 & 0 & 0 & 0 & 0 & 0 & 0 & 0 & 0.0 \\
\hline \hline
\end{tabular}

Table 8.5: Opposite-sign analysis of quark pair production $(b \bar{b}, c \bar{c})$ samples which involve $q \bar{q}$ creation from initial state gluon radiation. 


\begin{tabular}{|c|c|c|c|c|c|c|c|c|c|}
\hline & & & \multicolumn{6}{|c|}{ DILEPTON CUT } & \multirow{2}{*}{$\begin{array}{l}\text { Expected \# } \\
\text { Scaled by }\end{array}$} \\
\hline Bkgd & No. of & Lum & Lepton & Cosmic & Opp. & & & $N_{j}>10 \mathrm{GeV}$ & \\
\hline Sample & Events & $p b^{-1}$ & ID & Ray & Sign Isolation $M_{l l}$ & $E_{T} \quad \Delta \phi\left(\hat{E}_{T}, l\right)$ & $\Delta \phi\left(\hat{E}_{T}, j\right)$ & $01 \geq 2$ & Lep ID eff \\
\hline
\end{tabular}

$\bar{b}, c \bar{c}: 10.0 \leq p_{T} \leq 25.0$, final state gluon radiation

\begin{tabular}{|l|l|l|lllll|llll|lll|l|}
\hline CTEQ2L & 25000 & 104.3 & 0 & 0 & 0 & 0 & 0 & 0 & 0 & 0 & 0 & 0 & 0 & 0.0 \\
\hline \hline
\end{tabular}

$\bar{b}, c \bar{c}: 25.0 \leq p_{T} \leq 50.0$, final state gluon radiation

\begin{tabular}{|c|c|c|ccccc|cccc|ccc|c|}
\hline CTEQ3L & 100000 & 167.4 & 0 & 0 & 0 & 0 & 0 & 0 & 0 & 0 & 0 & 0 & 0 & 0.0 \\
GRV94LO & 100000 & 192.8 & 0 & 0 & 0 & 0 & 0 & 0 & 0 & 0 & 0 & 0 & 0 & 0.0 \\
\hline Total & 200000 & 360.2 & 0 & 0 & 0 & 0 & 0 & 0 & 0 & 0 & 0 & 0 & 0 & 0.0 \\
\hline \hline
\end{tabular}

$b \bar{b}, c \bar{c}: 50.0 \leq p_{T} \leq 500.0$, final state gluon radiation

\begin{tabular}{|c|c|c|ccccc|cccc|ccc|c|}
\hline CTEQ3L & 100000 & 321.3 & 7 & 7 & 7 & 0 & 0 & 0 & 0 & 0 & 0 & 0 & 0 & 0.0 \\
GRV94LO & 100000 & 389.9 & 8 & 8 & 6 & 0 & 0 & 0 & 0 & 0 & 0 & 0 & 0 & 0.0 \\
\hline Total & 200000 & 711.2 & 15 & 15 & 13 & 0 & 0 & 0 & 0 & 0 & 0 & 0 & 0 & 0.0 \\
\hline \hline
\end{tabular}

Table 8.6: Opposite-sign analysis of quark pair production $(b \bar{b}, c \bar{c})$ samples which involve $q \bar{q}$ creation from final state gluon radiation. 
successful event, we must attach an error based on the assumption that 1 event in a similar analysis would have passed, despite the fact that no events reached the $\mathbb{E}_{T}$ angular cuts or the 2 - jet filter stage. The error is determined by looking at the smallest subsample and then scaling 1 event by the ratio of the Run I integrated luminosity $\left(106.1 \mathrm{pb}^{-1}\right)$ to the corresponding luminosity of the sample.

The lowest momentum range samples, which produced no dilepton candidates, may be safely discarded from consideration. One might argue that the highest range samples would provide a more stringent limit as they are more capable of producing high- $p_{T}$ leptons. Yet in the case of direct $q \bar{q}$ production, the $25 \leq p_{T} \leq 50 \mathrm{GeV}$ samples yielded nearly as many candidate events at the lepton ID stage as the $50 \leq$ $p_{T} \leq 500 \mathrm{GeV}$ samples, because much larger samples are required to counteract the steeply falling differential cross-section as the quark momentum increases.

The direct production, ISR, and FSR samples are given equal consideration, so the smallest luminosity sample is $360.2 \mathrm{pb}^{-1}$ for final state gluon radiation in the $25 \leq$ $p_{T} \leq 50 \mathrm{GeV}$ range. A result of 1 event scaled by the ratio of luminosities corresponds to 0.29 events in Run I. This is an extremely conservative estimate considering that the efficiencies of the $\mathbb{E}_{T}$ and 2-jet cuts are largely ignored. Therefore, the expected number of background events from $b \bar{b}, c \bar{c}$ is $N_{b g}^{q \bar{q}}=0.00 \stackrel{+0.00}{+0.29}$.

\subsection{Drell-Yan}

Lepton pair production at hadron-hadron collisions was first described by Drell and Yan in 1970 [64]. Basically, Drell-Yan refers to the inelastic scattering process

$$
p^{+} p^{-} \longrightarrow l^{+} l^{-}+X
$$

as shown in Figure 8.2. A quark and an antiquark, one from each of the original partons, produce an intermediate boson which subsequently decays to a pair of leptons. The intermediate state may be a virtual photon or $Z$ boson. At the $Z^{0}$ resonant energy, a real vector boson will emerge. The other quark constituents of the proton and antiproton contribute to the underlying event structure. 


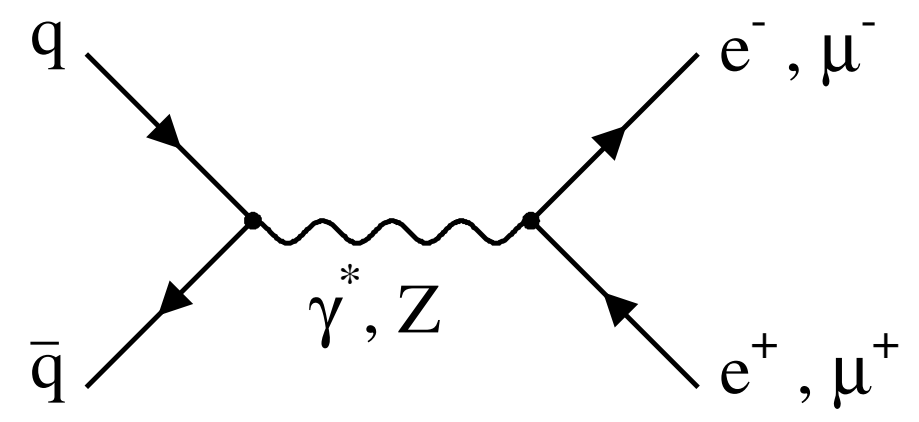

Figure 8.2: Leading order Feynman diagram for Drell-Yan production of lepton pairs in a hadronic collider event. Next-to-leading order forms include initial-state gluons which provide sufficient jet activity to pass the 2-jet cut. Here we consider only direct production of electrons and muons (although $\tau^{+} \tau^{-}$final production also contributes.

Higher-order diagrams involve initial state radiation of a gluon by one of the quarks, which may be sufficient to produce the necessary jet activity for such an event to pass the top dilepton analysis. Without any neutrinos in the event, the missing energy must stem from either uncounted jets or mismeasurement of lepton and jet energies. The magnitude and direction of the $\mathbb{E}_{T}$ must be such that they pass the selection cuts. In Drell-Yan events, the direction of the $\mathbb{E}_{T}$ tends to align with one of the jets, which was the original motivation for the $\Delta \phi\left(\mathbb{E}_{T}, j\right)$ cut. In the case where the leptons are taus $\left(Z^{0} \rightarrow \tau^{+} \tau^{-}\right)$, the $\mathbb{E}_{T}$ often points near the direction of one of the leptons and fails the $\Delta \phi\left(\not_{T}, l\right)$ cut.

The cross section for Drell-Yan is greatly enhanced at the $Z^{0}$ mass pole. The reconstructed dilepton mass for on-shell events ordinarily falls within the $Z^{0}$ mass window $75 \leq M_{l l} \leq 105 \mathrm{GeV}$, and only a relative few events remain after the invariant mass filter. As for the same-sign dilepton cut, Drell-Yan produces only opposite-sign lepton pairs. In order for a Drell-Yan event to enter the like-sign sample, either the charge of one lepton must be mismeasured or a jet must be misidentified as an electron.

The Drell-Yan samples used by the SUSY analysis group have generator-level cuts similar to those of the $b \bar{b}, c \bar{c}$ samples. The quark requirement was removed (for obvious 
reasons) while the lepton requirement was maintained: each event must contain at least one central lepton (electron or muon) with $p_{T}>9.0 \mathrm{GeV}$ and $|\eta|<1.5$, or two lower momentum leptons with $p_{T}>2.8 \mathrm{GeV}$ and $|\eta|<3.0$. Separate datasets were created for Drell-Yan from $\gamma$ and from $Z^{0}$. The virtual boson mass is limited to between 1 and $500 \mathrm{GeV}$, and the parton momentum is confined to the range 5-500 $\mathrm{GeV}$. An additional $Z^{0}$ sample with parton momentum range $[0.1,5]$ was also created. A summary of the same-sign analysis is presented in Table 8.7, while the results of an opposite-sign analysis of the same samples are shown for comparison in Table 8.8.

Of the thousands of events which pass the lepton ID and cosmic ray removal, only a small percentage are like-sign dilepton candidates: $0.03 \%$ of Drell-Yan $\gamma, 0.04 \%$ of $Z^{0}$, and $0.02 \%$ of low-momentum $Z^{0}$ events. For the 12 same-sign events that are observed, none survive past the missing energy cut. One event in the Drell-Yan $\gamma$ sample passes the $Z$ filter, yet has insufficient $\mathbb{E}_{T}$. More than half of the seven $Z^{0}$ events are removed by the invariant mass filter, and the others have insufficient $\mathbb{E}_{T}$ or fail the opening angle cut between $\mathbb{E}_{T}$ and a lepton. None of the low-momentum $Z^{0}$ events survive further than invariant mass stage. The opposite-sign analysis, on the other hand, yields a tiny percentage of events which pass all of the selection criteria: $0.003 \%$ of photon events and $0.005 \%$ of $Z$ boson events.

Since no like-sign events pass the analysis, the estimated number of background

events is $N_{b g}^{D-Y}=0.0$. The statistical error is calculated for each subsample, assuming that one event would have passed the analysis in a comparably sized sample. The estimated background, with error, for all three Drell-Yan samples is summarized in Table 8.9. For the overall estimate of the background contribution from Drell-Yan, we adopt the largest error of the three subsamples: $N_{b g}^{D-Y}=0.00{ }_{-0.00}^{+0.25}$.

\subsection{Dibosons $(W W, W Z, Z Z)$}

Vector bosons are produced at the Tevatron via processes such as those shown in Figure 8.3. The t-channel diagram on the left involves boson-fermion couplings, while the $s$-channel diagram on the right involves the tri-boson coupling. While only 


\begin{tabular}{|c|c|c|c|c|c|c|c|c|c|c|}
\hline & & & \multicolumn{7}{|c|}{ DILEPTON CUT } & \multirow{2}{*}{$\begin{array}{l}\text { Expected \# } \\
\text { Scaled by }\end{array}$} \\
\hline Bkgd & No. of & Lum & Lepton & Cosmic & Same & & & & $N_{j}>10 \mathrm{GeV}$ & \\
\hline Sample & Events & $p b^{-1}$ & ID & Ray & Sign & Isolation $M_{l l}$ & $E_{T} \quad \Delta \phi\left(\mathbb{E}_{T}, l\right)$ & $\Delta \phi\left(\boldsymbol{E}_{T}, j\right)$ & $\mid \begin{array}{lll}0 & 1 & 1\end{array}$ & Lep ID eff \\
\hline
\end{tabular}

Drell-Yan $\gamma: 5.0 \leq p_{T} \leq 500.0$

\begin{tabular}{|c|c|c|c|c|c|c|c|c|c|c|c|c|c|}
\hline CTEQ3L & 50000 & 241.3 & 842 & 838 & 0 & 0 & 0 & 0 & 0 & 0 & $\begin{array}{ll}0 & 0\end{array}$ & 0 & 0.0 \\
\hline GRV_94 & 50000 & 254.8 & 834 & 827 & 0 & 0 & 0 & 0 & 0 & 0 & $\begin{array}{ll}0 & 0\end{array}$ & 0 & 0.0 \\
\hline MRSD0' & 100000 & 431.8 & 1580 & 1577 & 1 & 1 & 1 & 0 & 0 & 0 & $\begin{array}{ll}0 & 0\end{array}$ & 0 & 0.0 \\
\hline Total & 200000 & 927.9 & 3256 & 3242 & 1 & 1 & 1 & 0 & 0 & 0 & $\begin{array}{ll}0 & 0\end{array}$ & 0 & 0.0 \\
\hline
\end{tabular}

Drell-Yan $Z^{0}: 5.0 \leq p_{T} \leq 500.0$

\begin{tabular}{|l|l|l|lllll|llll|lll|l|}
\hline CTEQ2L & 100000 & 424.8 & 16607 & 16527 & 7 & 7 & 3 & 1 & 0 & 0 & 0 & 0 & 0 & 0.0 \\
\hline \hline
\end{tabular}

Drell-Yan $Z^{0}: 0.1 \leq p_{T} \leq 5.0$

\begin{tabular}{|c|c|c|c|c|c|c|c|c|c|c|c|c|c|}
\hline CTEQ2L & 47047 & 184.0 & 7710 & 7535 & 2 & 1 & 0 & 0 & 0 & 0 & $\begin{array}{ll}0 & 0\end{array}$ & 0. & 0.0 \\
\hline MRSDO' & 50000 & 176.0 & 8463 & 8281 & 2 & 2 & 2 & 0 & 0 & 0 & $\begin{array}{ll}0 & 0\end{array}$ & 0. & 0.0 \\
\hline GRV LO & 50000 & 217.3 & 8149 & 7968 & 0 & 0 & 0 & 0 & 0 & 0 & $\begin{array}{ll}0 & 0\end{array}$ & 0. & 0.0 \\
\hline Total & 147047 & 577.3 & 24322 & 23784 & 4 & 3 & 2 & 0 & 0 & 0 & $\begin{array}{ll}0 & 0\end{array}$ & 0 & 0.0 \\
\hline
\end{tabular}

Table 8.7: Like-sign analysis of all Drell Yan $\gamma$ and $Z^{0}$ samples. 


\begin{tabular}{|c|c|c|c|c|c|c|c|c|c|}
\hline & & & \multicolumn{6}{|c|}{ DILEPTON CUT } & \multirow{2}{*}{$\begin{array}{c}\text { Expected \# } \\
\text { Scaled by }\end{array}$} \\
\hline Bkgd & No. of & Lum & Lepton Cosmic & Opp. & & & & $N_{j}>10 \mathrm{GeV}$ & \\
\hline Sample & Events & $p b^{-1}$ & Ray & Sign & Isolation $M_{l l}$ & $E_{T} \quad \Delta \phi\left(E_{T}, l\right)$ & $\Delta \phi\left(\mathbb{E}_{T}, j\right)$ & $\begin{array}{ll}0 & 1 \geq 2\end{array}$ & Lep ID eff \\
\hline
\end{tabular}

Drell-Yan $\gamma: 5.0 \leq p_{T} \leq 500.0$

\begin{tabular}{|c|c|c|ccccc|ccc|ccc|c|}
\hline CTEQ3L & 50000 & 241.3 & 842 & 838 & 838 & 802 & 666 & 14 & 8 & 6 & 1 & 2 & 3 & 2.6 \\
GRV_94 & 50000 & 254.8 & 834 & 827 & 827 & 802 & 634 & 10 & 7 & 4 & 3 & 0 & 1 & 0.9 \\
MRSD0' & 100000 & 431.8 & 1580 & 1577 & 1576 & 1504 & 1214 & 22 & 15 & 11 & 4 & 5 & 2 & 1.8 \\
\hline Total & 200000 & 927.9 & 3256 & 3242 & 3241 & 3108 & 2514 & 46 & 30 & 21 & 8 & 7 & 6 & 5.3 \\
\hline \hline
\end{tabular}

Drell-Yan $Z^{0}: 5.0 \leq p_{T} \leq 500.0$

\begin{tabular}{|l|l|l|llllllll|lll|l|}
\hline CTEQ2L & 100000 & 424.8 & 16607 & 16527 & 16520 & 16430 & 877 & 27 & 16 & 13 & 3 & 4 & 6 & 5.2 \\
\hline \hline
\end{tabular}

Drell-Yan $Z^{0}: 0.1 \leq p_{T} \leq 5.0$

\begin{tabular}{|c|c|c|ccccc|ccc|ccc|c|}
\hline CTEQ2L & 47047 & 184.0 & 7710 & 7535 & 7533 & 7526 & 367 & 1 & 0 & 0 & 0 & 0 & 0. & 0.0 \\
MRSD0' & 50000 & 176.0 & 8463 & 8281 & 8279 & 8276 & 413 & 0 & 0 & 0 & 0 & 0 & 0. & 0.0 \\
GRV LO & 50000 & 217.3 & 8149 & 7968 & 7968 & 7966 & 416 & 1 & 0 & 0 & 0 & 0 & 0. & 0.0 \\
\hline Total & 147047 & 577.3 & 24322 & 23784 & 23780 & 23768 & 1196 & 2 & 0 & 0 & 0 & 0 & 0 & 0.0 \\
\hline
\end{tabular}

Table 8.8: Opposite-sign analysis of all Drell Yan $\gamma$ and $Z^{0}$ samples. 


\begin{tabular}{|cc|}
\hline \hline Source & Run I estimated background \\
\hline Drell-Yan $\gamma$ & $0.00 \pm_{-0.00}^{+0.11}$ \\
Drell-Yan $Z^{0}$ & $0.00 \pm_{-0.00}^{+0.25}$ \\
Drell-Yan $Z^{0}\left(\right.$ low $\left.p_{T}\right)$ & $0.00 \pm_{-0.00}^{+0.18}$ \\
\hline \hline
\end{tabular}

Table 8.9: Summary of the expected Drell-Yan contribution to the same-sign dilepton signal. The errors are statistical only, based on the assumption one event would have passed the analysis despite the fact that no events passed the $\mathbb{E}_{T}$ cuts.
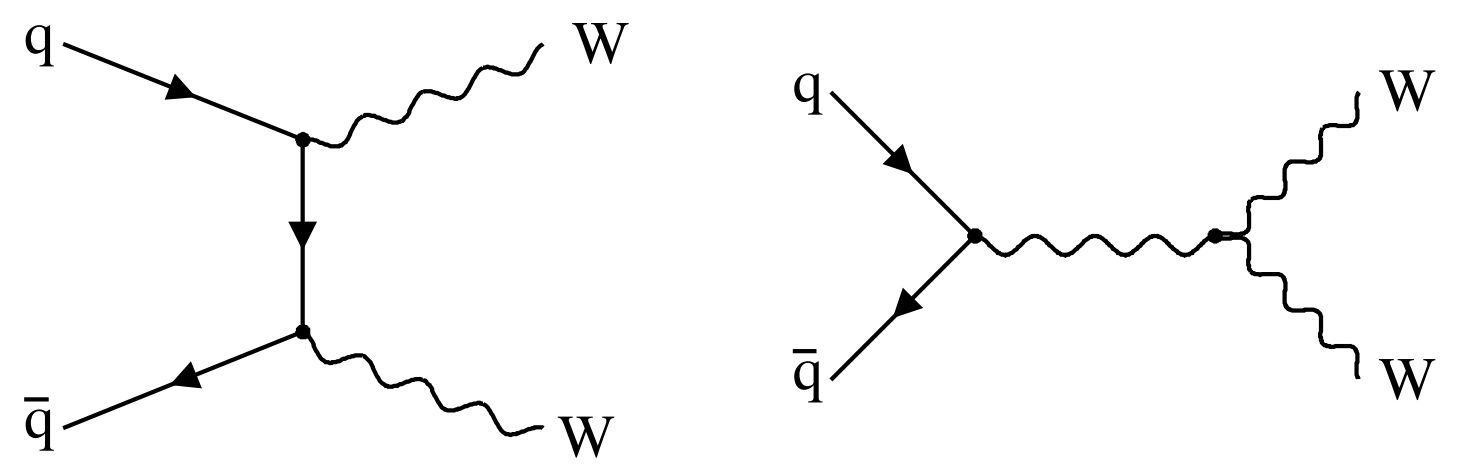

Figure 8.3: Feynman diagram for $W W$ diboson production. Analogous diagrams exist for $W Z$, while $Z Z$ production proceeds via a diagram analogous to the left one involving boson-fermion couplings.

$W W$ production is shown, similar diagrams exist for $W Z$ production. $Z Z$ cannot be produced from any Standard Model tri-boson vertex but do arise from a diagram analogous to the first in Figure 8.3 involving boson-fermion couplings. Both $W W$ and $W Z$ receive contributions from $W W Z$ and $W W \gamma$ couplings, which are the only triboson vertices in the Standard Model. Next-to-leading-order calculation [65, 66, 67] of the cross sections for diboson production in $p \bar{p}$ collisions at $\sqrt{s}=1.8 \mathrm{TeV}$ yields: $\sigma(p \bar{p} \rightarrow W W)=9.5 \mathrm{pb}$ and $\sigma(p \bar{p} \rightarrow W Z)=2.5 \mathrm{pb}$.

Diboson events involving $W W$ enter the dilepton channel when both $W$ 's decay leptonically $\left(p \bar{p} \rightarrow W^{+} W^{-} \rightarrow l^{+} \nu_{l} l^{-} \bar{\nu}_{l}\right)$. As with the top dilepton channel, the 
branching ratio for $W W$ to dileptons $(l=e$ or $\mu)$ is $5 \%$. $W \rightarrow \tau \nu_{\tau}$ will also contribute through leptonic tau decays, at the level of $\sim 10 \%$. Once again, lepton charge mismeasurement is required for the event to pass the like-sign analysis. The two neutrinos in the event provide a sufficient amount of missing energy, yet higher-order diagrams involving gluon radiation or underlying event processes are necessary to supply the required jet activity to pass the $2-j$ et filter.

In order for a $W Z$ event to pass the dilepton criteria, the $Z^{0}$ must decay leptonically to produce the two high $p_{T}$ leptons, while the $W$ must decay hadronically to create the 2 jets. The branching ratio for $Z^{0}$ to dielectrons or dimuons is $6.8 \%$ and for $W$ to $u d$ or $c s$ is $67 \%$, so the combined BR to this channel is $\approx 4.5 \%$. The invariant mass filter removes a large percentage of $W Z$ events because the two leptons originate from the $Z$ boson. This process does not provide a source of missing energy, which must come from mismeasurement of the jets instead. Leptonic decay of both the $W$ and $Z^{0}$ will augment the contribution of $W Z$ to the dilepton channel. However, the branching ratio is three times smaller, and the jets would have to come from QCD processes, further reducing the effective cross section for this decay mode.

The cross section for $Z^{0} Z^{0}$ production is more than a factor of two smaller than for $W^{ \pm} Z^{0}: \sigma(p \bar{p} \rightarrow Z Z)=1.0 \mathrm{pb}[68] . Z Z$ events produce a dilepton signature through one leptonic and one hadronic $Z$ decay, and the branching ratio for this mode is $9 \%$. Large rejection factors are expected for the $\mathbb{E}_{T}$ and $Z$ mass filters because there is no natural source of missing energy and the dilepton pair from $Z$ often reconstructs to form an invariant mass in the $Z$ window. To this, we add the requirement that the same-sign signature be due to charge mismeasurement. The resulting product of a small cross section, low branching ratio into the 2 leptons +2 jets channel, and low efficiency for passing the selection criteria yields the smallest expected contribution of the three diboson background processes.

Monte Carlo samples were produced for all three diboson processes using ISAJET. The results obtained from the same-sign and opposite-sign dilepton analyses of these simulated events are presented in Tables 8.10 and 8.11. Although the $W W$ and $Z Z$ samples correspond to integrated luminosities of 2.3 and $6.8 \mathrm{fb}^{-1}$, respectively, no same-sign dilepton candidates emerge in the $W W$ sample, while none of $11 Z Z$ 


\begin{tabular}{|c|c|c|c|c|c|c|c|c|c|}
\hline & & & \multicolumn{6}{|c|}{ DILEPTON CUT } & \multirow{2}{*}{$\begin{array}{l}\text { Expected \# } \\
\text { Scaled by }\end{array}$} \\
\hline Bkgd & No. of & Lum & Lepton & Cosmic & Same & & & $N_{j}>10 \mathrm{GeV}$ & \\
\hline Sample & Events & $p b^{-1}$ & ID & Ray & Sign & Isolation $M_{l l}$ & $\mathbb{E}_{T} \quad \Delta \phi\left(\mathbb{E}_{T}, l\right) \Delta \phi\left(\mathbb{E}_{T}, j\right)$ & $\begin{array}{lll}0 & 1 & \geq 2\end{array}$ & Lep ID eff \\
\hline
\end{tabular}

Diboson $W W: 0.1 \leq p_{T} \leq 500.0$

\begin{tabular}{|l|l|l|lllll|llll|llll|l|}
\hline CTEQ2L & 15000 & 2343.0 & 171 & 170 & 0 & 0 & 0 & 0 & 0 & 0 & 0 & 0 & 0 & 0.0 \\
\hline
\end{tabular}

Diboson $W Z: 0.1 \leq p_{T} \leq 500.0$

\begin{tabular}{|l|l|l|lllll|llll|llll|l|}
\hline CTEQ2L & 5000 & 5819.0 & 100 & 100 & 17 & 17 & 7 & 5 & 5 & 5 & 3 & 1 & 1 & 0.9 \\
\hline \hline
\end{tabular}

Diboson ZZ: $0.1 \leq p_{T} \leq 500.0$

\begin{tabular}{|l|l|l|lllll|llll|llll|l|}
\hline CTEQ2L & 5000 & 6843.0 & 164 & 164 & 11 & 10 & 0 & 0 & 0 & 0 & 0 & 0 & 0 & 0.0 \\
\hline \hline
\end{tabular}

Table 8.10: Like-sign analysis of diboson $W W, W Z$ and $Z Z$ samples.

\begin{tabular}{|c|c|c|c|c|c|c|c|c|}
\hline & & & \multicolumn{5}{|c|}{ DILEPTON CUT } & \multirow{3}{*}{$\begin{array}{l}\text { Expected \# } \\
\text { Scaled by } \\
\text { Lep ID eff }\end{array}$} \\
\hline Bkgd & No. of & Lum & Lepton & Cosmic & Opp. & & $N_{j}>10 \mathrm{GeV}$ & \\
\hline Sample & Events & $p b^{-1}$ & ID & Ray & Sign Isolation $M_{l l}$ & $\mathbb{E}_{T} \quad \Delta \phi\left(\mathbb{E}_{T}, l\right) \quad \Delta \phi\left(\mathbb{E}_{T}, j\right)$ & $\begin{array}{lll}0 & 1 & 1\end{array}$ & \\
\hline
\end{tabular}

Diboson $W W: 0.1 \leq p_{T} \leq 500.0$

\begin{tabular}{|l|l|l|lllll|llll|lll|l|}
\hline CTEQ2L & 15000 & 2343.0 & 171 & 170 & 170 & 168 & 151 & 114 & 108 & 106 & 67 & 2.3 & 16 & 14.3 \\
\hline \hline
\end{tabular}

Diboson WZ: $0.1 \leq p_{T} \leq 500.0$

\begin{tabular}{|l|l|l|lllll|lllll|lll|l|}
\hline CTEQ2L & 5000 & 5819.0 & 100 & 100 & 88 & 87 & 6 & 4 & 4 & 4 & 1 & 3 & 0 & 0.0 \\
\hline \hline
\end{tabular}

Diboson ZZ: $0.1 \leq p_{T} \leq 500.0$

\begin{tabular}{|l|l|l|lllll|llll|lll|l|}
\hline CTEQ2L & 5000 & 6843.0 & 164 & 164 & 163 & 159 & 4 & 1 & 1 & 1 & 1 & 0 & 0 & 0.0 \\
\hline \hline
\end{tabular}

Table 8.11: Opposite-sign analysis of diboson $W W, W Z$ and $Z Z$ samples. 


\begin{tabular}{|cc|}
\hline \hline Source & Run I estimated background \\
\hline Diboson $W^{+} W^{-}$ & $0.00_{-0.00}^{+0.05}$ \\
Diboson $W^{ \pm} Z^{0}$ & $0.02 \stackrel{0.02}{+0.00}$ \\
Diboson $Z^{0} Z^{0}$ & $0.00 \stackrel{-0.00}{+0.02}$ \\
\hline \hline
\end{tabular}

Table 8.12: Summary of the expected diboson contribution to the same-sign dilepton signal. The errors are statistical only. For the $W W$ and $Z Z$ backgrounds, the errors are based on the assumption that for similar sized samples, one event would have passed the analysis.

events pass the isolation and invariant mass filters.

As for the $W Z$ sample, five like-sign events pass all of the missing energy criteria, and one event meets the 2 - jet requirement. (The opposite-sign analysis of the $W Z$ sample also yields 4 events which pass the $\mathbb{E}_{T}$ cuts, although none of these dileptons are accompanied by two or more jets.) After correcting for the lepton ID efficiency, this results in an acceptance rate of 0.9 events in $5.8 \mathrm{fb}^{-1}$. Once this is scaled down to Run I luminosity, though, the estimated number of events from $W Z$ is only 0.02 .

Table 8.12 summarizes the expected background contributions from all diboson sources. The low statistical errors are attributable to the fact that, because the cross sections involved are small, each diboson sample size of only 5000 events is equivalent to nearly 20-60 times the luminosity collected during Run I.

\section{5 tt $t \bar{t}$ production}

Standard Model top quark production also contributes to the like-sign dilepton background through the semileptonic decay of the $b$ quark. The majority of dilepton events from top are produced by the leptonic decay of both $W$ bosons. However, a small percentage originate from the semileptonic mode $\left(W_{1} \rightarrow l \nu, W_{2} \rightarrow j j\right)$, where the second lepton is created by leptonic decay one of the bottom quarks. 
In the top analysis, calculation of the opposite-sign dilepton acceptance using Monte Carlo found that after all selection cuts, $(86 \pm 3) \%$ of the remaining events come from $W W \rightarrow l l,(11 \pm 1) \%$ are from $W W \rightarrow l \tau \rightarrow l l$, and $(3 \pm 1) \%$ are from $W W \rightarrow \operatorname{ljj}(b, c \rightarrow l)$. (Here $l$ refers to either an electron or muon.) Given the top analysis result of 9 candidate events minus a background of $2.1 \pm 0.4$ events, a rough estimate of the contribution from semileptonic $b, c$ decay would be on the order of 0.2 events. Because there is no charge preference as to whether $b(c)$ or $\bar{b}(\bar{c})$ provides the lepton in this dilepton mode, we should expect the same contribution to the like-sign channel.

Two ISAJET Monte Carlo samples were created for calculation of the like-sign background estimate from $t \bar{t}$ : one with initial- and final-state gluon radiation simulated and the other without. The former is believed to more accurately emulate top quark production, while the latter is mainly for comparison purposes. Both samples contain 25,000 events, corresponding to integrated luminosities of $5.5 \mathrm{fb}^{-1}$.

The results of the same-sign and opposite-site analyses of these samples are presented in Tables 8.13 and 8.14. Any events which pass all of the selection criteria are scaled by the lepton ID efficiency correction factors. After adjusting the results to account for the MC sample luminosities, the expected number of like-sign dilepton events in Run I is $0.28 \pm 0.07$ for the primary $t \bar{t}$ sample and $0.39 \pm 0.09$ for the control sample (which ignores the effects of gluon radiation).

\subsection{Fake leptons}

The methods developed for lepton identification, jet identification, photon removal, and so forth are simply sets of kinematic cuts designed to aid in identifying particles from the information collected by the detector. While generally effective, they are not fully efficient at determining exactly which type of physics object produced a given signal. The possibility that the reconstruction software may occasionally be fooled must be considered when examining the potential sources of background events.

Fake leptons are hadronic particles which leave a signature in the detector similar 


\begin{tabular}{|c|c|c|c|c|c|c|c|c|c|c|}
\hline \multirow[b]{2}{*}{ Bkgd } & \multirow[b]{2}{*}{ No. of } & \multirow[b]{2}{*}{ Lum } & \multicolumn{7}{|c|}{ DILEPTON CUT } & \multirow{2}{*}{$\begin{array}{l}\text { Expected \# } \\
\text { Scaled by }\end{array}$} \\
\hline & & & Lepton & Cosmic & Same & & & & $N_{j}>10 \mathrm{GeV}$ & \\
\hline Sample & Events & $p b^{-1}$ & ID & Ray & Sign & Isolation $M_{l l}$ & $\mathbb{E}_{T} \quad \Delta \phi\left(\mathbb{E}_{T}, l\right)$ & $\Delta \phi\left(\mathbb{E}_{T}, j\right)$ & $\begin{array}{lll}0 & 1 & \geq 2\end{array}$ & Lep ID eff \\
\hline
\end{tabular}

$t \bar{t}: 0.1 \leq p_{T} \leq 500.0, m_{t}=175 \mathrm{GeV}$

\begin{tabular}{|l|l|l|lllll|llll|lll|l|}
\hline CTEQ3L & 25000 & 5430.0 & 411 & 411 & 44 & 36 & 30 & 24 & 22 & 20 & 0 & 1 & 19 & 14.1 \\
\hline \hline
\end{tabular}

$t \bar{t}: 0.1 \leq p_{T} \leq 500.0, m_{t}=175 \mathrm{GeV}$, No gluon radiation

\begin{tabular}{|l|l|l|lllll|llll|lll|l|}
\hline CTEQ3L & 25000 & 5466.0 & 470 & 470 & 44 & 37 & 34 & 30 & 29 & 29 & 0 & 1 & 28 & 19.9 \\
\hline \hline
\end{tabular}

Table 8.13: Like-sign analysis of $t \bar{t}$ samples, with and without gluon radiation incorporated into the ISAJET simulations.

\begin{tabular}{|c|c|c|c|c|c|c|c|c|c|}
\hline & & & \multicolumn{6}{|c|}{ DILEPTON CUT } & \multirow{3}{*}{$\begin{array}{l}\text { Expected \# } \\
\text { Scaled by } \\
\text { Lep ID eff }\end{array}$} \\
\hline Bkgd & No. of & Lum & Lepton & Cosmic & Opp. & & & $N_{j}>10 \mathrm{GeV}$ & \\
\hline Sample & Events & $p b^{-1}$ & ID & Ray & Sign Isolation $M_{l l}$ & $\mathbb{E}_{T} \quad \Delta \phi\left(\mathbb{E}_{T}, l\right)$ & $\Delta \phi\left(\mathbb{E}_{T}, j\right)$ & $0 \quad 1 \geq 2$ & \\
\hline
\end{tabular}

$t \bar{t}: 0.1 \leq p_{T} \leq 500.0, m_{t}=175 \mathrm{GeV}$

\begin{tabular}{|l|l|l|lllll|llll|lll|l|}
\hline CTEQ3L & 25000 & 5430.0 & 411 & 411 & 369 & 348 & 312 & 277 & 261 & 243 & 2 & 24 & 217 & 185.2 \\
\hline \hline
\end{tabular}

$t \bar{t}: 0.1 \leq p_{T} \leq 500.0, m_{t}=175 \mathrm{GeV}$, No gluon radiation

\begin{tabular}{|l|l|l|lllll|llll|lll|l|}
\hline CTEQ3L & 25000 & 5466.0 & 470 & 470 & 433 & 412 & 348 & 309 & 285 & 264 & 1 & 20 & 243 & 208.3 \\
\hline \hline
\end{tabular}

Table 8.14: Opposite-sign analysis of $t \bar{t}$ samples, with and without gluon radiation incorporated into the ISAJET simulations. 
to that of an electron or muon. A jet which contains a photon or neutral pion $\left(\pi^{0}\right)$ has the potential to deposit sufficient electromagnetic energy in the calorimeter to mimic the signal of an electron. A jet which penetrates the hadronic calorimeter and reaches the muon chambers may be misidentified as a muon. Also, hadronic decays within the tracking chamber or calorimeter can produce muons. Generally, most of these are vetoed by the impact parameter and z-vertex cuts, but occasionally one will appear to originate from the primary vertex.

When dealing with other background sources, the primary process of interest has generally been capable of producing most of the same-sign dilepton event topology. On occasion, we have relied somewhat on the ability of a jet to fake a lepton or other mismeasurement effects, but this has been in the context of enhancing the contribution. In this section, we are examining processes which would not ordinarily reproduce the top dilepton signature at all, and the only way in which such an event would pass the analysis is if one or more jets are misidentified as leptons. The probability of a jet faking a lepton is already small $\left(\sim 10^{-4}\right)$, so the possibility of an event containing two fakes is quite remote. Therefore, we only consider sources which naturally produce one real lepton and require only one fake.

For the top dilepton analysis, the largest contribution to the fake rate was expected from $W+\geq 3$ jet events. As shown in Figure 8.4, a $W$ boson is produced in conjunction with three or more jets from the gluon and final state gluon radiation. If the $W$ decays leptonically and one of the jets fakes a second lepton, the event may be accepted by the dilepton analysis. When a jet fakes a lepton, there is no preference for which charge will be measured, so it is equally likely to produce an opposite-sign or like-sign pair. Therefore, any estimate of the background from fakes for the opposite-sign top dilepton analysis is equally applicable to any like-sign analysis using the same cuts, including our gluino search.

The equal contribution to opposite-sign and like-sign channels was not lost upon the top dilepton authors. In attempting to estimate the contribution of fakes, they made use of the number of same-sign events observed in the data, regardless of whether they may be attributed to fake leptons, another background source, or a signal of new physics. Since these events are considered signal in this analysis, the 


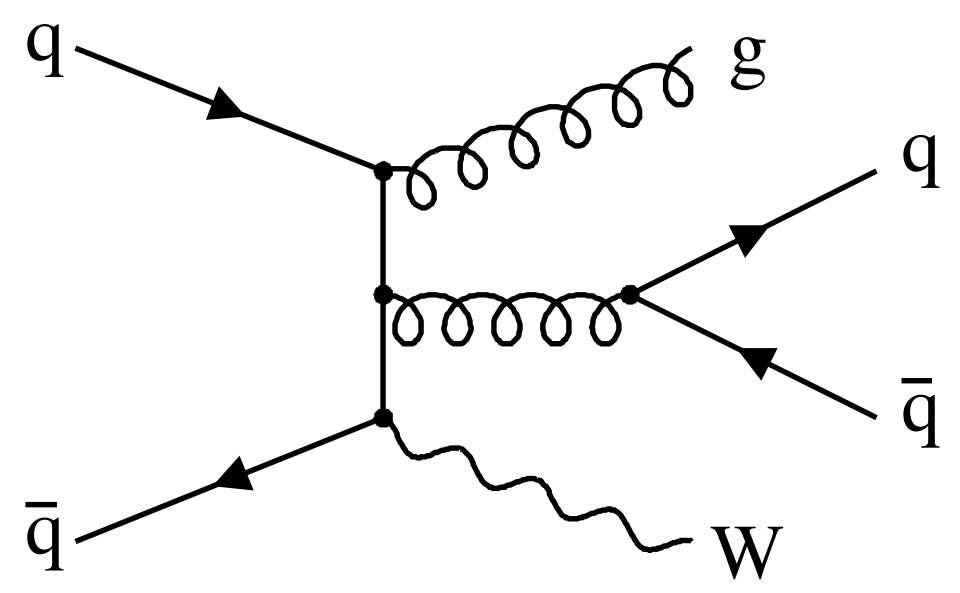

Figure 8.4: Feynman diagram for $W$ boson production associated with 3 or more jets.

estimate obtained via their method is not applicable. For the sake of completeness, we describe it here anyway.

Fake leptons should contribute to all jet multiplicities, not just the $\geq 2$ jets bin. In the data, there are two same-sign $e \mu$ events before the final 2-jet cut, and both events pass. One may safely assume that the 2-jet cut is not truly $100 \%$ efficient and that this is just a reflection of the low statistical sample. In order to calculate the true efficiency of the 2-jet cut for fake lepton events, they calculated the ratio of the number of $W+\geq 2$ jets $+\geq 1$ fakeable jet events to the number of $W+\geq 1$ fakeable jet events. A jet is labelled fakeable if it has $p_{T} \geq 20 \mathrm{GeV}$ and $|\eta|<1.2$. The $W^{ \pm}$is identified by a tight lepton plus $\mathbb{E}_{T} \geq 25 \mathrm{GeV}$ (from the neutrino).

From Run I data, the 2-jet cut efficiency calculated by this method is $0.12 \pm 0.01$. Multiplying this by the two same-sign $e \mu$ events in the data before the jet multiplicity cut gives an estimate of

$$
N_{b g}^{f a k e}=0.24 \pm 0.17 \text { events (Same-sign events method) }
$$

for the background contribution from fakes.

An alternate method, which does not rely on the same-sign events in the data, yields a similar result. A more detailed description of this method is available elsewhere $[69,70]$. In this case, the inclusive jet samples described in Section 3.8 were 


\begin{tabular}{|c|cc|}
\hline \hline Source & Before 2-jet cut & After 2-jet cut \\
\hline$e e+\mu \mu$ & $1.5 \pm 1.2$ & $0.21 \pm 0.17$ \\
$e \mu$ & $1.1 \pm 1.1$ & $0.16 \pm 0.16$ \\
\hline$l l$ & $2.6 \pm 1.9$ & $0.37 \pm 0.23$ \\
\hline \hline
\end{tabular}

Table 8.15: Estimated contribution from fake lepton events for any opposite-sign or like-sign top dilepton analysis in Run I, using the "fakeable jet" probability and $W+$ multijet sample. The number of events from fakes is shown after all dilepton selection cuts except for the 2 - jet cut and after all cuts.

used. In these samples, real leptons from $W^{ \pm}$and $Z^{0}$ decays are first removed. Leptons from heavy flavor quark decays are not removed due to the uncertainty on the $b$-jet fraction in the samples. By not removing this source of real leptons, the background estimate obtained using this method is an overestimate. Once the probability of a jet faking a lepton has been determined from the inclusive jet samples, it is then applied to the number of "fakeable" jets found in $W+\geq 3$ jet events.

The results are broken down in Table 8.15, showing the expected number of events in Run I before and after the final 2-jet cut. Given these numbers, the 2-jet cut efficiency is closer to 0.14 . The total number of background events expected from fakes using this method is

$$
N_{b g}^{\text {fake }}=0.37 \pm 0.23 \text { events } \quad(W+\geq 3 \text { jets method }) .
$$

This result is more conservative and is independent of the number of observed likesign events in the data. As mentioned earlier, the fake rate is equally applicable to either the opposite-sign or like-sign dilepton analysis. Therefore, we shall adopt this as our estimate of the background contribution as well. 


\subsection{Background contribution summary}

All of the background sources discussed in the previous sections are summarized in Table 8.16. We estimate the total contribution of all backgrounds to the same-sign top dilepton channel to be

$$
N_{b g}^{\text {total }}=0.67+0.24
$$

For many of the backgrounds, like-sign analysis of our Monte Carlo samples failed to yield any events which passed all of the dilepton requirements. In some cases, the process failed to populate any of the selection stages two or three steps removed from the final stage or produced no like-sign events at the lepton ID stage. The uncertainties attached to each individual background estimate are therefore statistics limited. Additional efforts to increase the sample sizes would reduce the errors even further. The primary contributors to the overall background, $t \bar{t}$ and fake leptons, will benefit from future optimization studies of the isolation and identification requirements.

For this analysis, reduction of the background estimate would not improve the reach of our analysis. The limiting factor is the number of observed events (3) compared to the background contribution, which was suspected to be much less than 1 event before the analysis was carried out and which has now been verified. Unless there exists another process which contributes to the same-sign dilepton channel whether it be a Standard Model interaction, detector effect, or possibly even Supersymmetry - the discrepancy between the Run I observation and the background estimate are somewhat difficult to reconcile. A continuation of this analysis in Run II will hopefully shed further light on this question. 


\begin{tabular}{|cc|}
\hline \hline Source & Run I estimated background \\
\hline$b \bar{b}, c \bar{c}$ & $0.00 \pm 0.29$ \\
Drell-Yan & $0.00 \pm 0.25$ \\
Diboson (WW) & $0.00 \pm 0.00$ \\
Diboson (WZ) & $0.02 \pm 0.02$ \\
Diboson (ZZ) & $0.00 \pm 0.02$ \\
$t \bar{t}$ & $0.28 \pm 0.07$ \\
fake leptons & $0.37 \pm 0.23$ \\
\hline Total & $0.67 \pm 0.24$ \\
\hline \hline
\end{tabular}

Table 8.16: Estimated contribution to the same-sign top dilepton channel from all Standard Model background sources. The statistical errors are added in quadrature. 


\section{Chapter 9}

\section{Results and Conclusions}

We observe 3 same-sign events in $106.1 \mathrm{pb}^{-1}$ of data collected during Run I at CDF: $2 e \mu$ dilepton events and 1 trilepton event $(e \mu \mu)$. The event acceptance has been calculated for a range of proposed gluino and squark masses using Monte Carlo, and the systematic uncertainty on the acceptance is estimated to be $10 \%$. The expected contribution from background sources is $0.67_{-0.24}^{+0.55}$. From this information, we proceed to calculate a cross section limit on $\tilde{g} \tilde{g}$ production and determine the extent of exclusion in $m_{\tilde{g}}-m_{\tilde{q}}$ parameter space. Subsequently, we shall discuss the relevance of this analysis for future experiments and make a few concluding remarks.

\subsection{Statistical analysis}

In a single-channel analysis without backgrounds, the number of observed signal events $\left(n_{0}\right)$ is related to the average number of expected signal events $(\mu)$ through a Poisson distribution. The probability $P$ of observing exactly $n$ events when $\mu$ events are expected is given by

$$
P\left(n_{0} \mid \mu\right)=\frac{\mu^{n} e^{-\mu}}{n !}
$$

For a fixed value of $\mu$, the total probability of observing $n_{0}$ or fewer events is simply the summation up to $n_{0}$ of the probabilities given by Eq. 9.1:

$$
\epsilon=\sum_{n=0}^{n_{0}} P(n \mid \mu) .
$$


When performing a search for new particles, the value of $\mu$ is the unknown variable to be determined using the measurement $n_{0}$. In order to claim the absence of a signal and exclude some portion of parameter space, we need to find the upper limit on the expected number of events, $N$. This is defined as the value of $\mu$ for which some finite probability $\epsilon$ (usually 5 or $10 \%$ ) exists that a similarly performed random experiment would observe $n_{0}$ or fewer events. The confidence level (CL) in the upper limit, defined as $(1-\epsilon)$, and represents the probability that a random experiment would observe more than $n_{0}$ events. It is, in effect, a measure of the certainty that the number of observed events reflects a true absence of signal rather than a statistical fluctuation downward. In practice, $\mu$ is varied until the value of $\epsilon$ is obtained corresponding to the desired confidence level, and $N=\mu_{\text {upperlimit }}$.

If background processes contribute to the signal, Eq. 9.2 is modified slightly to become [71]

$$
\epsilon=\frac{\sum_{n=0}^{n_{0}} P\left(n \mid \mu_{B}+N\right)}{\sum_{n=0}^{n_{0}} P\left(n \mid \mu_{B}\right)}
$$

where $\mu_{B}$ is the average number of background events expected among the $n_{0}$ observed. The Poisson upper limit $N$ becomes the number of expected signal events in the measurement. The probability $(1-\epsilon)$ represents the probability that a random experiment would produce more than $n_{0}$ events and have $n_{B} \leq n_{0}$, where $n_{B}$ is the number of background events contained in the sample. The denominator in Eq. 9.3 makes $\epsilon$ a conditional probability and excludes non-physical values by ensuring that $N$ remains positive. Past editions of the PDG Review of Particle Properties have noted that this results in a "conservative" upper limit in that for some true $\mu_{S}$, the probability of observing $N>\mu_{S}$ events actually exceeds $(1-\epsilon)$, on average.

Complications arise when attempting to fold the effect of systematic errors into the upper limit calculation. No generally accepted method exists within the particle physics community for dealing with errors in the acceptance and background calculations, and varying approaches have been developed to suit the needs of each experiment. CDF has adopted a method which involves a Bayesian-style integration 
over the uncertainties [72]. One assumes a Gaussian distribution about the true values of $\mu_{B}$ and $\mu_{S}$ using the values and widths obtained through Monte Carlo studies. Equation 9.3 is expanded to include integrations over these Gaussian distributions in $\mu_{B}$ and $\mu_{S}$, and $N$ becomes the Poisson upper limit on $\mu_{S}$ such that:

$$
\epsilon=\frac{\sum_{n=0}^{n_{0}} \frac{1}{2 \pi \sigma_{N} \sigma_{B}} \int_{0}^{\infty} \int_{0}^{\infty} P\left(n \mid \mu_{B}^{\prime}+\mu_{S}^{\prime}\right) e^{-\frac{\left(\mu_{B}-\mu_{B}^{\prime}\right)^{2}}{2 \sigma_{B}^{2}}} e^{-\frac{\left(N-\mu_{S}^{\prime}\right)^{2}}{2 \sigma_{N}^{2}}} d \mu_{B}^{\prime} d \mu_{S}^{\prime}}{\sum_{n=0}^{n_{0}} \int_{0}^{\infty} P\left(n \mid \mu_{B}\right) e^{-\frac{\left(\mu_{B}-\mu_{B}^{\prime}\right)^{2}}{2 \sigma_{B}^{2}}} d \mu_{B}^{\prime}}
$$

where:

- $\mu_{B}=$ expected number of background events

- $\sigma_{B}=$ Gaussian uncertainty on $\mu_{B}$

- $A=$ event acceptance rate

- $\sigma_{N}=N \sigma_{A} / A: \sigma_{A}$ is the overall uncertainty on $A$ and $\sigma_{A} / A$ is the relative uncertainty on $\mu_{S}$.

The integral in Eq. 9.4 is evaluated using a Monte Carlo integration technique rather than through direct integration. For each test value of $N$, a large ensemble of pseudoexperiments is generated, generally 10,000-100,000 or more depending on the desired accuracy. Each pseudoexperiment is assigned an expected number of signal and background events $\left(\mu_{S}\right.$ and $\left.\mu_{B}\right)$ according to Gaussian distributions, then Poisson-distributed numbers of signal $\left(n_{S}\right)$ and background $\left(n_{B}\right)$ events are generated. In our case, where the error on the background estimate is asymmetric, the distribution is modified accordingly. For those pseudoexperiments where $n_{B} \leq n_{0}$, the fraction in which $n_{B}+n_{S}>n_{0}$ is denoted by $f$, which is equivalent to the confidence level for the test value of $N$. Successive iterations vary $N$ over smaller and smaller ranges until the desired confidence level $(\mathrm{CL}=1-\epsilon)$ is obtained and $N$ is measured to within the desired precision.

Using the results of our analysis - 3 observed events, a $10 \%$ relative uncertainty on the acceptance, and an estimate of $0.677_{-0.24}^{+0.55}$ background events - we calculate 
an upper limit of $N=7.19$ expected signal events at $95 \%$ confidence level. The event acceptance rate for $\tilde{g} \tilde{g}$ events is in the range of $3.75 \times 10^{-3}$ to $4.50 \times 10^{-3}$ for our main sequence of $m_{\tilde{g}}-m_{\tilde{q}}$ pairings (the first 13 samples in Table 6.9), with an average acceptance of $4.04 \times 10^{-3}$. This translates into upper limits on the $\tilde{g} \tilde{g}$ cross section in the range $\sigma_{\tilde{g} \tilde{g}}^{\text {limit }}=15.1-18.0 \mathrm{pb}$, with an average of $16.8 \mathrm{pb}$. The full results are shown in Table 9.1.

In Figure 9.1 we have combined the theoretical curve for the $\tilde{g} \tilde{g}$ cross section from PROSPINO with the limit curve obtained from the Monte Carlo samples. Using the average cross section limit of $16.8 \mathrm{pb}$, the limit curve may be extrapolated to lower values of $m_{\tilde{g}}$ than shown in Figure 9.1, and the potential exists to exclude the gluino mass up to approximately 181 or $182 \mathrm{GeV}$. However, a limit in the mass region $m_{\tilde{g}} \approx m_{t}$ using the $\tilde{g} \rightarrow t \tilde{t}$ decay mode is difficult to support. For nearly degenerate gluino and top quark masses, the $\tilde{t}$ mass is forced to be unnaturally light $\left(m_{\tilde{t}}<6-7\right.$ $\mathrm{GeV}$ in this case). To claim an exclusion in this corner of parameter space would require a detailed phenomenology study. Additional Monte Carlo studies would be necessary to reduce the influence of statistical errors on the acceptance and to further study the sources of systematic error. In the view of all within the analysis group, the benefits of pursuing this course were far outweighed by the extensive effort that would have been required. Therefore, at present we cannot place any limits upon the allowed gluino mass using the Run I dataset.

Of the various factors which enter into the limit calculation, one of the more damaging was the total of 3 observed events. Out of curiosity, we also estimate the reach of the same-sign dilepton analysis had there been only 1 observed event. If we maintain the expected background contribution of $0.67{ }_{-0.24}^{+0.55}$ events, the upper limit on the number of expected signal events would have been $N=4.35$ at $95 \%$ confidence level, which is $39 \%$ less than our result of $N=7.19$. The average cross section limit would have been $\sigma_{\tilde{g} \tilde{g}}^{\text {limit }}($ avg $)=10.1 \mathrm{pb}$, and the analysis would have excluded the gluino mass up to approximately $196 \mathrm{GeV}$. In this case, the $\tilde{t}$ and $\tilde{\chi}_{1}^{0}$ masses would still be required to be light, yet would lie in the more palatable region of $m<21$ $\mathrm{GeV}$. A comparison of the limit curves obtained for the data (3 observed events) and for this theoretical case (1 observed event) is shown in Figure 9.2. 


\begin{tabular}{|c|c|c|c|c|c|c|c|}
\hline \multicolumn{4}{|c|}{ MC SAMPLE INFO } & \multirow{2}{*}{$\begin{array}{l}\text { \# of events } \\
\text { passing } \\
\text { LS analysis }\end{array}$} & \multirow{2}{*}{$\begin{array}{l}\text { \# of events } \\
\text { scaled by } \\
\text { lep ID eff }\end{array}$} & \multirow{2}{*}{$\begin{array}{c}\text { Event } \\
\text { acceptance } \\
\text { rate }\end{array}$} & \multirow{2}{*}{$\begin{array}{l}\text { Upper } \\
\text { limit } \\
\sigma(\mathrm{pb})\end{array}$} \\
\hline$m_{\tilde{g}}$ & $m_{\tilde{t}}$ & $m_{\tilde{\chi}_{1}^{0}}$ & $\begin{array}{l}\text { No. of } \\
\text { Events }\end{array}$ & & & & \\
\hline 200 & 20 & 15 & 148483 & 677 & 582.3 & 0.00392 & 17.3 \\
\hline 210 & 30 & 20 & 148165 & 672 & 566.2 & 0.00382 & 17.8 \\
\hline 220 & 40 & 30 & 150000 & 665 & 563.2 & 0.00375 & 18.0 \\
\hline 230 & 50 & 30 & 150000 & 698 & 585.2 & 0.00390 & 17.4 \\
\hline 240 & 60 & 40 & 150000 & 709 & 596.3 & 0.00398 & 17.0 \\
\hline 250 & 70 & 50 & 150000 & 722 & 608.0 & 0.00405 & 16.7 \\
\hline 260 & 80 & 60 & 150000 & 730 & 615.4 & 0.00410 & 16.5 \\
\hline 270 & 90 & 70 & 150000 & 705 & 594.9 & 0.00397 & 17.1 \\
\hline 280 & 100 & 80 & 150000 & 720 & 609.4 & 0.00406 & 16.7 \\
\hline 290 & 110 & 90 & 150000 & 733 & 616.3 & 0.00411 & 16.5 \\
\hline 300 & 120 & 100 & 145579 & 738 & 617.2 & 0.00424 & 16.0 \\
\hline 310 & 130 & 110 & 148992 & 732 & 617.1 & 0.00414 & 16.4 \\
\hline 320 & 140 & 120 & 150000 & 798 & 674.9 & 0.00450 & 15.1 \\
\hline 230 & 20 & 15 & 148662 & 721 & 617.0 & 0.00415 & 16.3 \\
\hline 250 & 40 & 30 & 148165 & 788 & 669.0 & 0.00452 & 15.0 \\
\hline 280 & 70 & 50 & 150000 & 822 & 693.6 & 0.00462 & 14.7 \\
\hline 310 & 100 & 80 & 148998 & 773 & 650.2 & 0.00436 & 15.5 \\
\hline 280 & 40 & 30 & 147828 & 800 & 676.8 & 0.00458 & 14.8 \\
\hline 310 & 70 & 50 & 150000 & 851 & 717.4 & 0.00478 & 14.2 \\
\hline
\end{tabular}

Table 9.1: Upper limit on $\sigma_{\tilde{g} \tilde{g}}$ calculated from the acceptances for the various Monte Carlo samples. 
Cross section and number of events in $106.1 \mathrm{pb}^{-1}$

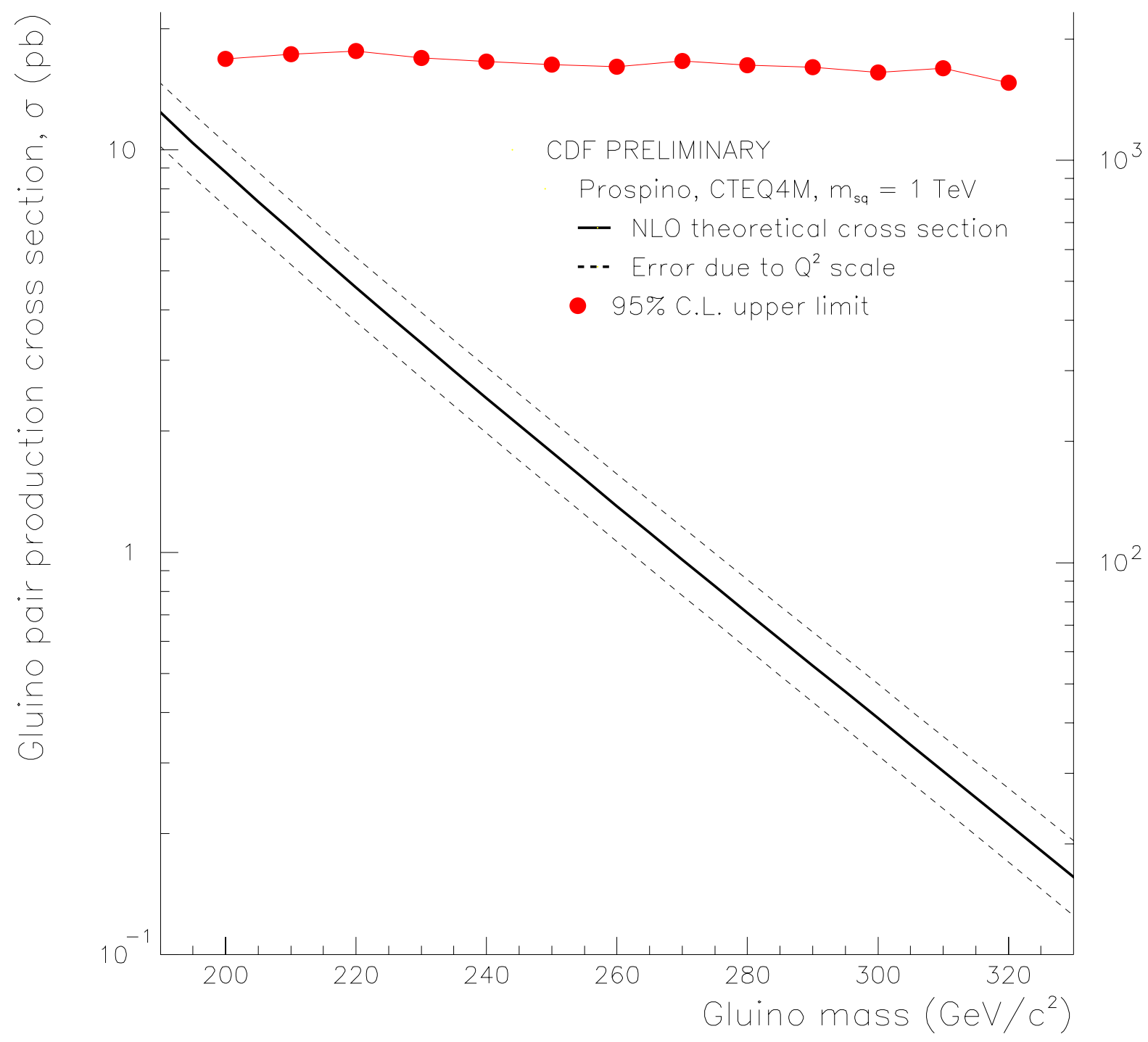

Figure 9.1: 95\% Confidence Level limits on $\tilde{g} \tilde{g} \rightarrow(t \tilde{t})(t \tilde{t})$ compared to the next-toleading order (NLO) theoretical calculation of $\sigma_{\tilde{g} \tilde{g}}$ from PROSPINO. 
Cross section and number of events in $106.1 \mathrm{pb}^{-1}$

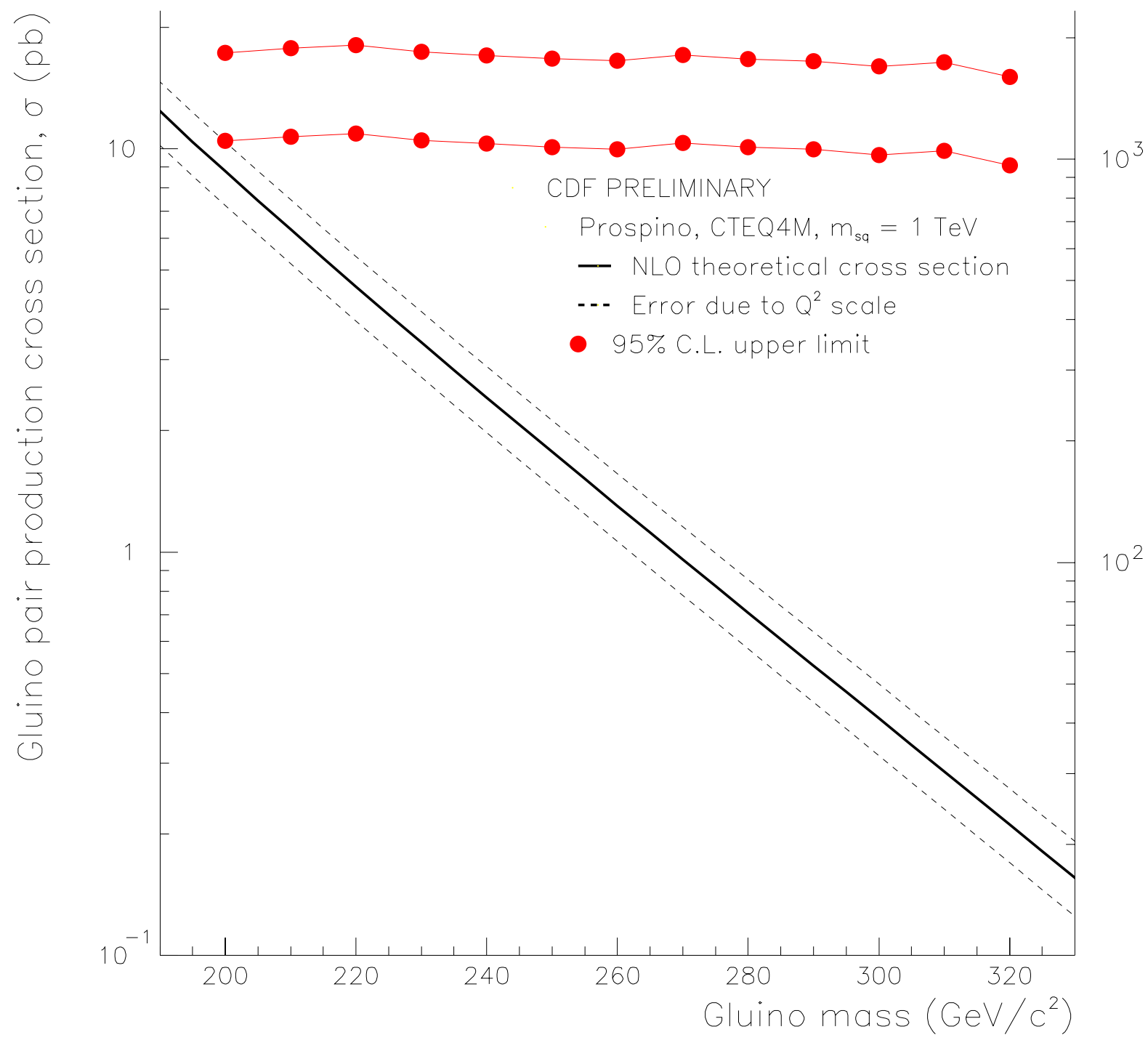

Figure 9.2: The upper limit curve is the Run I limit, based on the 3 observed events in the data. The lower limit curve is based on the presumption of only 1 observed event in $106.1 p b^{-1}$. In the second case, the extrapolated limit on $m_{\tilde{g}}$ would have been $\approx 196 \mathrm{GeV}$. 


\subsection{Prospects for Run II}

Despite the inability to set a limit on the gluino mass in Run I, the same-sign dilepton analysis promises to be a fairly competitive channel for the discovery of Supersymmetry in Run II. Accelerator and detector upgrades improve several aspects of the production and detection of gluino pair events, while modifications of the analysis technique should greatly benefit the event acceptance while simplifying some of the procedures used in the past.

For Run II, the Tevatron accelerator energy has been ramped up from $1.8 \mathrm{TeV}$ to $1.96 \mathrm{TeV}$. This $9 \%$ increase in center-of-mass energy translates into a greater cross section for gluino pair production. In Figure 9.3, we show the NLO cross section calculation from PROSPINO for $p \bar{p}$ collisions at $E_{c m}=1.8$ and $1.96 \mathrm{TeV}$, along with error curves to account for the dependence of $\sigma_{\tilde{g} \tilde{g}}$ upon the choice of $Q^{2}$ scale. Figure 9.4 shows in greater detail the ratio of the two cross sections, reflecting a $35-70 \%$ increase in the cross section over the range of gluino masses from 200 to 320 $\mathrm{GeV}$.

The Tevatron has been redesigned to operate at higher luminosity, $1 \times 10^{32} \mathrm{~cm}^{-2} \mathrm{sec}^{-1}$. The bunch spacing has been reduced, first to $396 \mathrm{~ns}$ and ultimately to $132 \mathrm{~ns}$. The expectation for Run II is to collect at least $2 \mathrm{fb}^{-1}$ of data during the first stage of operation (2001-2004), which gives a factor of 20 increase in total integrated luminosity over Run I. The additional statistics vastly improve the ability to perform precision studies and searches for rare decays. Whereas Run I offered on the order of a few hundred $\tilde{g} \tilde{g}$ events (resulting in a handful of dilepton events at best), the potential for several thousand $\tilde{g} \tilde{g}$ events exists in Run II, and anywhere from dozens to hundreds of same-sign top dilepton events could be detected. Beyond Run II is the prospect of continued operation of the Tevatron at higher luminosity from 2005 to 2008 and beyond, resulting in the accumulation of up to $15-30 \mathrm{fb}^{-1}$ of data during Run IIb.

Several upgrades and improvements to the CDF detector have been driven by the Run II accelerator design. The silicon vertex detector (SVX II) [73] has expanded to 5 layers of double-sided sensors, which will provide stereo hit information. One side of each silicon wafer consists of the familiar $r-\phi$ strips of the SVX, while the other 
Dependence of cross section on p $\bar{p}$ collision energy

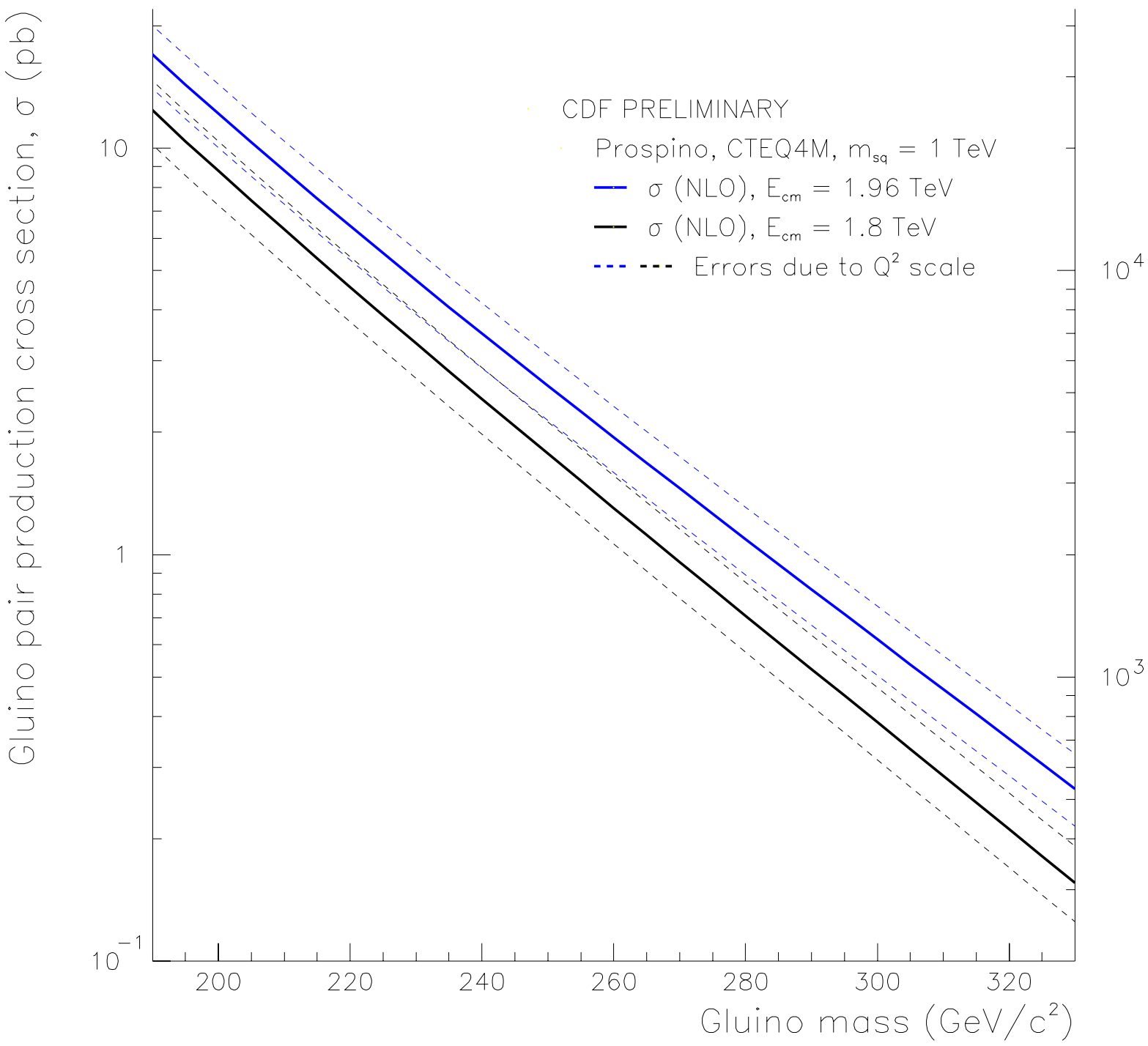

Figure 9.3: Comparison of the Run I and Run II NLO cross sections for gluino pair production $\left(\sigma_{\tilde{g} \tilde{g}}\right)$ at the Tevatron. The increase in center of mass energy from 1.8 $\mathrm{TeV}$ to $1.96 \mathrm{TeV}$ boosts $\sigma_{N L O}$ by $35-70 \%$ over the range of gluino masses shown. The scale at right shows the corresponding number of $p \bar{p} \rightarrow \tilde{g} \tilde{g}+X$ events expected to be produced in $2 \mathrm{fb}^{-1}$ of luminosity during the first stage of Run II. 
Comparison of Run II/Run I gluino-gluino cross sections

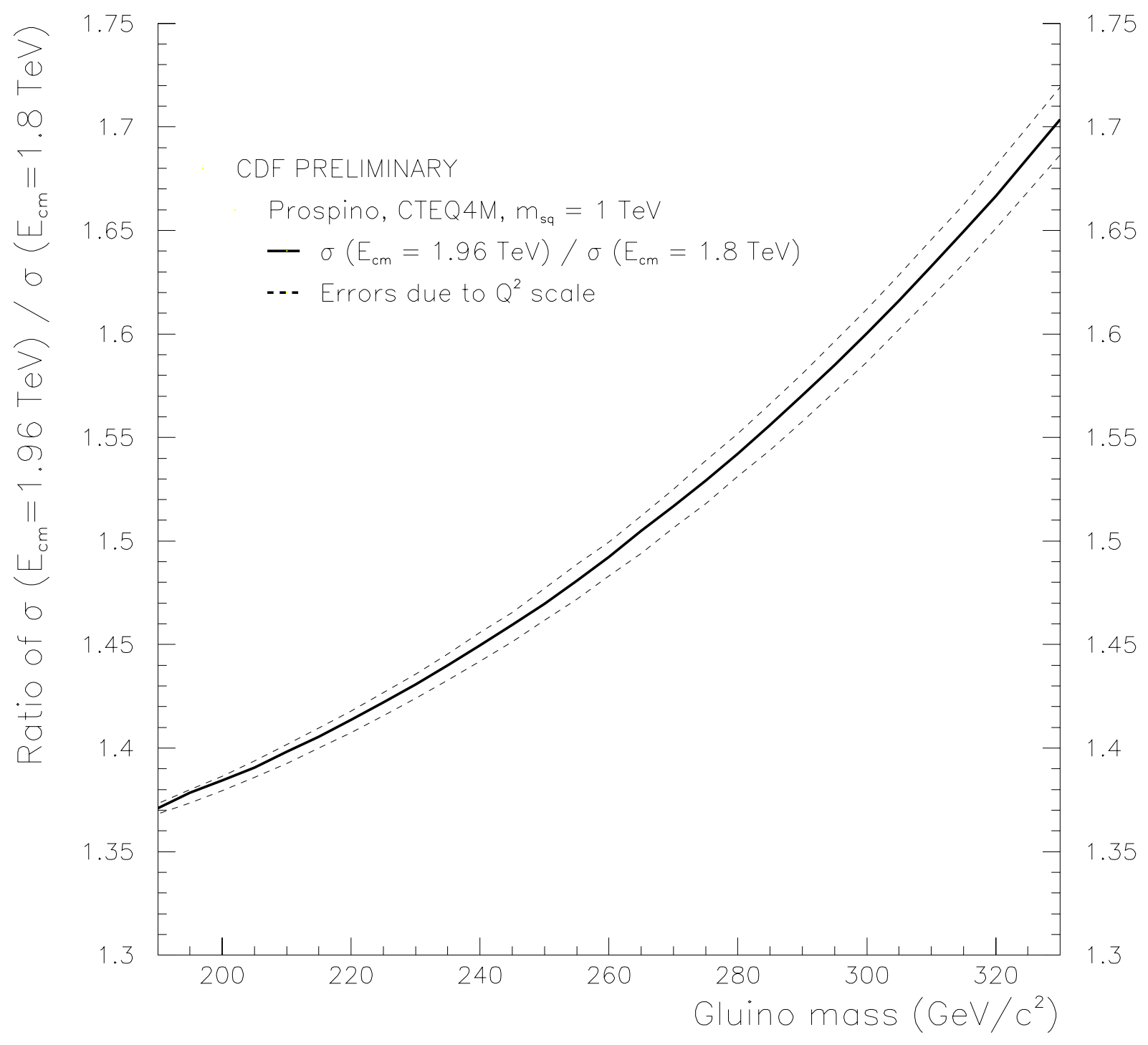

Figure 9.4: Comparison of the cross section for gluino pair production at Run I energy $\left(E_{c m}=1.8 \mathrm{TeV}\right)$ and Run II energy $\left(E_{c m}=1.96 \mathrm{TeV}\right)$. 
side adds either $90^{\circ}$ or "small-angle" $\left( \pm 1.2^{\circ}\right)$ stereo strips. The three-barrel design is nearly a meter long and extends coverage of the interaction region out to $2.5 \sigma$, or pseudorapidity $|\eta|<2$. The intermediate silicon detector (ISL) adds a sixth and seventh layer to provide additional coverage in the region $1<|\eta|<2$. Momentum resolution $\left(\delta p_{T} / p_{T}^{2}\right)$ in the central region will remain $<0.1 \%$ and will be $<0.4 \%$ for $1<|\eta|<2$. 3-D reconstruction of SVX tracks will allow for impact parameter resolution of $\sigma_{r \phi}<30 \mu \mathrm{m}$ and $\sigma_{z}<60 \mu \mathrm{m}$ for central high- $p_{T}$ tracks. Table 9.2 details the improvements to the silicon system for Run II.

The tracking and muon coverage have expanded from $|\eta|<1$ out to $|\eta|<2$. This translates into an increase in electron acceptance of $33 \%$, while acceptance will increase by $12 \%$ for muons from top decays and by $30 \%$ for muons from $W^{ \pm}$and $Z^{0}[74]$. At the beginning of Run II, the muon trigger will be inclusive up to $|\eta|<1.2$, while the ultimate goal is to include $|\eta|<1.5$.

On the analysis front, a working group at CDF has come together to develop criteria for the Run II high- $p_{T}$ dilepton dataset [75]. Among the improvements that have been suggested:

- An inclusive high- $p_{T}$ dilepton sample would be created from the inclusive electron and muon samples. Events would be required to have a second lepton passing a set of selection cuts, though no topological requirements such as lepton sign, $\mathscr{E}_{T}$, or $H_{T}$ (explained below) would be applied.

- The top dilepton sample would be a direct subset of the inclusive dilepton sample. Additional cuts will closely follow those of the original analysis: two leptons with $E_{T}\left(p_{T}\right)>20 \mathrm{GeV}$ for electrons (muons); $\not_{T}>25 \mathrm{GeV} ; 2$ or more jets.

- There is a proposal to remove the $\mathbb{E}_{T}$ cut for $e \mu$ events and to include a significance requirement for $e e$ and $\mu \mu$ events. The goal is to better distinguish between top and Drell-Yan events. Approximately 27\% of top events are rejected by the current mass window filter $\left(75<M_{l l}<105 \mathrm{GeV}\right)$ on ee and $\mu \mu$ candidates, resulting in the loss of $\approx 1$ signal event in $100 p b^{-1}[76]$. While 


\begin{tabular}{|c|c|c|}
\hline Detector parameters & SVX' & SVX II \\
\hline Readout coordinates & $r-\phi$ & $r-\phi, r-z$ \\
\hline Number of barrels & 2 & 3 \\
\hline Number of layers per barrel & 4 & 5 \\
\hline Number of wedges per barrel & 12 & 12 \\
\hline Ladder length & 25.0 & $29.0 \mathrm{~cm}$ \\
\hline Combined detector length & $51 \mathrm{~cm}$ & $87 \mathrm{~cm}$ \\
\hline Radius of innermost layer & $2.86 \mathrm{~cm}$ & $2.44 \mathrm{~cm}$ \\
\hline Radius of outermost layer & $7.87 \mathrm{~cm}$ & $10.6 \mathrm{~cm}$ \\
\hline$r-\phi$ readout pitch $(\mu m)$ & $60 ; 60 ; 60 ; 55$ & $60 ; 62 ; 60 ; 60 ; 65$ \\
\hline$r-z$ readout pitch $(\mu m)$ & - & $141 ; 125.5 ; 60 ; 141 ; 65$ \\
\hline Stereo angle of $r-z$ strips & - & $90^{\circ} ; 90^{\circ} ; 1.2^{\circ} ; 90^{\circ} ; 1.2^{\circ}$ \\
\hline Length of readout channel $(r-\phi)$ & $25.0 \mathrm{~cm}$ & $16.0 \mathrm{~cm}$ \\
\hline$r-\phi$ readout chips per ladder & $2 ; 3 ; 4 ; 6$ & $4 ; 6 ; 10 ; 12 ; 14$ \\
\hline$r-z$ readout chips per ladder & - & $4 ; 6 ; 10 ; 8 ; 14$ \\
\hline$r-\phi$ readout channels & 46,080 & 211,968 \\
\hline$r-z$ readout channels & - & 193,536 \\
\hline Total number of channels & 46,080 & 405,504 \\
\hline Total number of readout chips & 360 & 3168 \\
\hline Total number of detectors & 288 & 720 \\
\hline Total number of ladders & 96 & 180 \\
\hline
\end{tabular}

Table 9.2: Comparison of the mechanical specifications for the SVX' (Run I) and SVX II (Run II) detectors. 

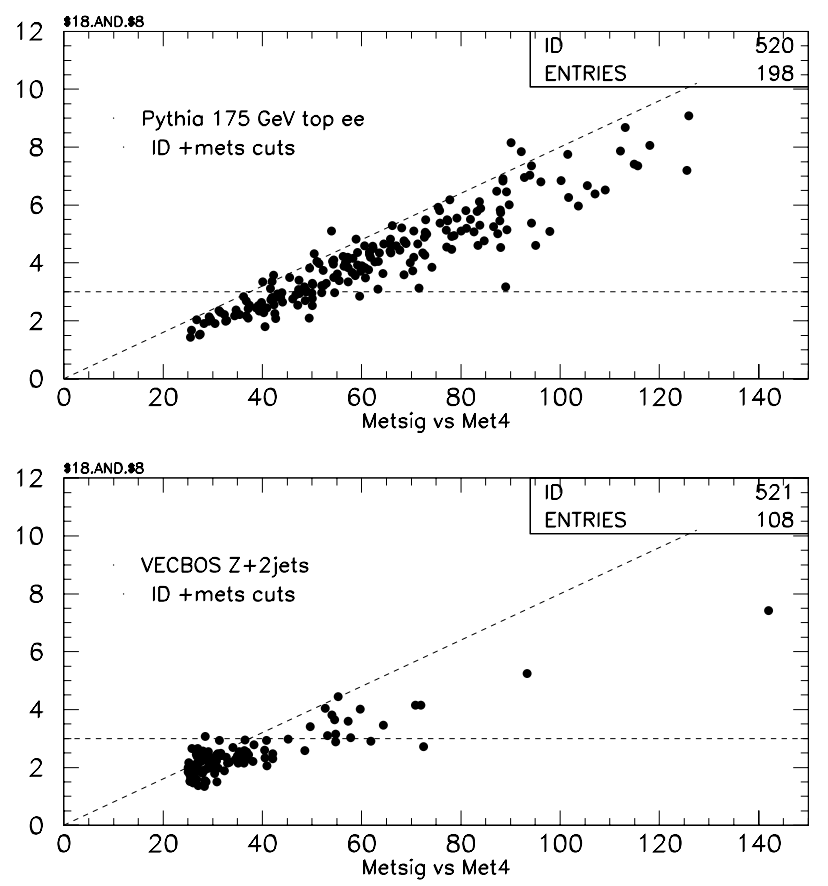

Figure 9.5: The missing $E_{T}$ significance is plotted versus $\mathbb{E}_{T}$ for dielectrons from $t \bar{t}$ in PYTHIA Monte Carlo (top) and from $Z^{0}+2$ jet background in VECBOS (bottom). In both cases, the lepton ID selection and standard missing $E_{T}$ cuts have been applied. The diagonal line and line at $\phi=3$ are for reference only. (Taken from Ref. [76].)

$t \bar{t}$ events have two stiff neutrinos as an intrinsic source of missing energy, $Z^{0}$ backgrounds do not. The missing $E_{T}$ significance is defined as

$$
\phi=\mathbb{E}_{T} / \sqrt{\Sigma E_{T}}
$$

This new variable offers an extra handle for separating top signal from background, as seen in Figure 9.5. For the Drell-Yan background, there are many events with large $\mathbb{E}_{T}$ but low significance. Further studies are required to optimize the combination of $M_{l l}, \mathbb{E}_{T}$, and $\phi$ cuts, but the background rejection factor can be maintained while acceptance of top events is increased.

- Another kinematic variable to be adopted from other analyses is $H_{T}$, the scalar sum of all transverse energy in the event. This is the summation of all $E_{T}$ for 


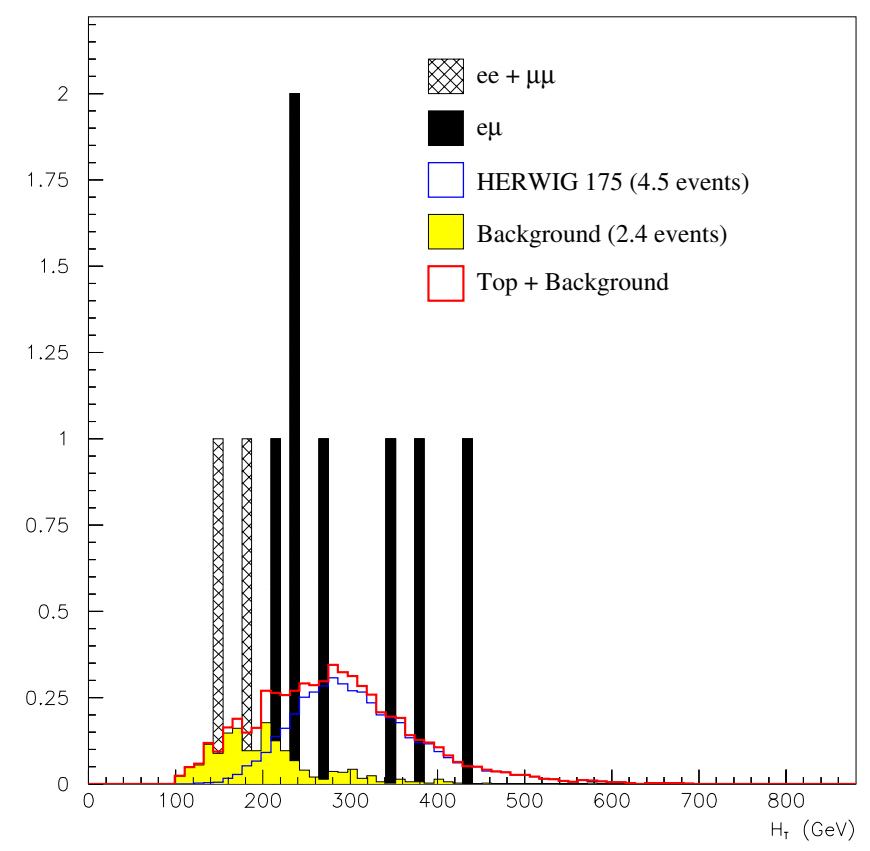

Figure 9.6: Monte Carlo distributions of $H_{T}$ for dilepton events from $t \bar{t}$ and background processes which pass the Run I analysis criteria. The Run I data are also shown. (Taken from Ref. [75].)

electrons, $p_{T}$ for muons, corrected jet $E_{T}$ (for all jets with $E_{T}^{\text {uncorr }}>10 \mathrm{GeV}$ and $|\eta|<2.0$ ), and $\mathbb{E}_{T}$ (corrected for muons and jets). A cut of $H_{T}>200 \mathrm{GeV}$ is expected to remove approximately $50 \%$ of remaining background after all Run I cuts have been applied while removing less than $5 \%$ of $t \bar{t}$. The distribution of $H_{T}$ for Monte Carlo $t \bar{t}$ and background dilepton events is shown in Figure 9.6, with a histogram overlay of the 9 opposite-sign top dilepton events from Run I.

- Jets will be required to have $E_{T}^{\text {corr }}>15 \mathrm{GeV}$, up from $10 \mathrm{GeV}$ in Run I, and must be centrally located $(|\eta|<2.0)$.

- The distinction between tight and loose lepton categories will be removed. The entire procedure adds complexity to dilepton analyses, especially in background calculations, and contributes a relatively small gain in acceptance. Instead, 
efforts will be made to further optimize the cuts.

- The cut requiring that each lepton be associated with a particular vertex and the constraint that both leptons be assigned to the same vertex would be applied at the end of the analysis. Currently this is incorporated into the initial lepton selection.

- No requirements would be applied to the trigger path beyond those triggers used to obtain the inclusive electron and muon samples. The trigger system is fully efficient for high- $p_{T}$ leptons with $E_{T}\left(p_{T}\right)>20 \mathrm{GeV}$, a fact which we made use of in this analysis.

- The non-signal combinations of SS/OS and number of jets will be used as control samples for the understanding of backgrounds, especially the contributions from fake leptons and dibosons.

Additional minor changes to the electron and muon selection criteria have been proposed for a short-term "Summer 2002" analysis, which would concentrate on the first $200 p b^{-1}$ of integrated luminosity or all data collected by April 1, 2002, whichever comes first. This will allow for presentation of early results at the summer conferences. Long-term plans are for the dilepton analysis to include plug electrons. This increases the acceptance significantly compared to Run I, where the absence of sufficient tracking information at high $\eta$ made detection and identification difficult. Studies are currently underway to determine the necessary cuts and efficiencies for plug leptons using $Z^{0}$ and $W^{ \pm}$events. Initial results show that a sizable gain is possible.

The series of upgrades to the accelerator (higher luminosity and larger cross sections), the detector (larger coverage, improved triggers for inclusive electron and muon samples, sophisticated tracking), and the analysis techniques (optimization of selection cuts, etc.) translate into a far greater potential for the same-sign dilepton analysis to detect the signal of gluino production in Run II than existed in Run I. The exact magnitude of improvement is difficult to predict and depends upon multiple factors. Having said that, Figure 9.7 is an attempt to quantify the reach of this analysis in Run II. The Run I event acceptances and the upper limit on the 
number of expected signal events $(N)$ have been converted into a cross section limit using the anticipated Run II luminosity. Our estimate is that after an initial $2 f b^{-1}$ integrated luminosity, the same-sign dilepton analysis has the potential to discover gluino production for masses up to $\sim 290 \mathrm{GeV}$.

\subsection{Conclusions}

In this analysis, we have searched for gluino pair production in $p \bar{p}$ collisions at $\sqrt{s}=1.8 \mathrm{TeV}$ during Run I at the Tevatron. Under the assumption that $B R(\tilde{g} \rightarrow$ $t \tilde{t})=1$, we searched for the like-sign top dilepton signature $\tilde{g} \tilde{g}+X \rightarrow(t \tilde{t})(t \tilde{t})+X \rightarrow$ $l^{ \pm} l^{ \pm}+X$. Based on the 3 observed events and expected background of $0.677_{-0.24}^{+0.55}$, we have calculated a range of cross-section limits of $\sigma_{\tilde{g} \tilde{g}}^{\text {limit }}=15.1-18.0 \mathrm{pb}$ for $200 \leq m_{\tilde{g}} \leq 320 \mathrm{GeV}$, with an average cross-section limit on gluino pair production, $\sigma_{\tilde{g} \tilde{g}}^{\text {limit }}(a v g)$, of $16.8 \mathrm{pb}$. This corresponds to an approximate gluino mass of 181$182 \mathrm{GeV}$. We do not attempt to exclude any portion of parameter space due to the uncertainties associated with the region $m_{\tilde{g}} \approx m_{t}$. If analysis of the data had yielded 1 signal event, the estimated mass limit would have been $m_{\tilde{g}}>196 \mathrm{GeV}$.

The observed excess of signal over the background expectation is large enough to prevent exclusion of any gluino mass consistent with $m_{\tilde{g}}>m_{t}+m_{\tilde{t}}$, yet it is not sufficient to claim the presence of gluino pair production in Run I at the Tevatron. This result may simply be due to an upward fluctuation of the Standard Model background or may result from some new process which is yet to be fully understood. Whether or not we have observed preliminary evidence of physics beyond the Standard Model is a question for future experiments to determine. Regardless of the answer, the samesign top dilepton channel holds much interest for those searching for Supersymmetry in Run II. 
Cross section and number of events in $2 \mathrm{fb}^{-1}$

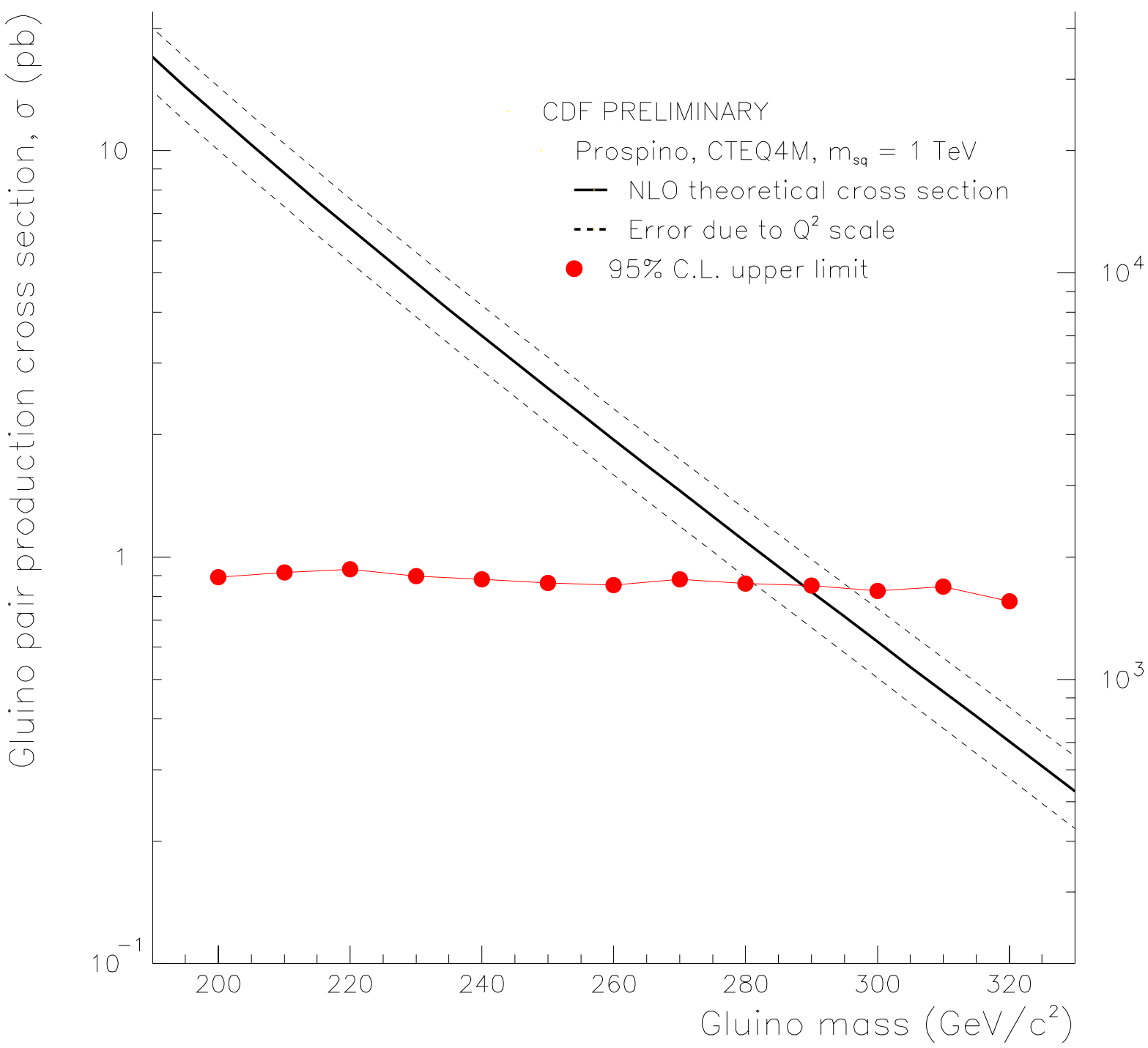

Figure 9.7: Rough estimate of the potential for the same-sign dilepton analysis for setting a limit on $m_{\tilde{g}}$ in Run II $\left(2 f b^{-1}\right)$. This assumes the same conditions (observed signal and estimated background) as those used to calculate the Run I limit. 


\section{Bibliography}

[1] T. Affolder, et al. Search for a Fourth Generation Quark More Massive than the $Z^{0}$ Boson in $p \bar{p}$ Collisions at $\sqrt{s}=1.8 \mathrm{TeV}$. Phys. Rev. Lett., 84:835, 2000.

[2] Particle Data Group; D.E. Groom, et al. Review of Particle Physics. Eur. Phys. J., C15:1, 2000. The most recent results from the LEP Electroweak Working Group may be found at http://lepewwg.web.cern.ch/LEPEWWG/ .

[3] Stephen P. Martin. A Supersymmetry Primer. hep-ph/9709356, 1998.

[4] J. Wess, J. Bagger. Supersymmetry and Supergravity, Princeton University Press, 1983; P. Fayet, S. Ferrara. Phys. Rep. 32:249, 1977; P. West. Introduction to Supersymmetry and Supergravity, World Scientific, 1986; R. N. Mohapatra. Unification and Supersymmetry, Springer-Verlag, 1986.

[5] E. Witten. Nucl. Phys., B188:513, 1981. N. Sakai. Z. Phys. C., 11:153, 1981; S. Dimopoulos, H. Georgi. Nucl. Phys., B193:150, 1981; R.K. Kaul, P. Majumdar. Nucl. Phys., B199:36, 1982.

[6] H.P. Niles. Phys. Rep., 110:1, 1984; H.E. Haber, G.L. Kane. Phys. Rep., 117:75, 1985.

[7] D. Freedman, S. Ferrara, P. van Nieuwenhuizen. Phys. Rev. D, 13:3214, 1976; S. Deser, B. Zumino. Phys. Lett. B, 62:335, 1976; D. Freedman, P. van Nieuwenhuizen. Phys. Rev. D, 14:912, 1976.

[8] R.M. Barnett, J.F. Gunion, H.E. Haber. Discovering Supersymmetry with Like Sign Dileptons. Phys. Lett., B315:349, 1993. 
[9] Particle Data Group; D.E. Groom, et al. Review of Particle Physics. Eur. Phys. J., C15:1, 2000. For limits on the gluino, stop, neutralino, and degenerate squark masses, refer to the section on "Supersymmetric Particle Searches."

[10] G.L. Kane, S. Mrenna. Do About Half the Top Quarks at FNAL Come From Gluino Decays?. hep-ph/9605351, 1996.

[11] P. Azzi, et al. Observation of $t \bar{t}$ Production in the All-Hadronic Channel. CDF/ANAL/TOP/CDFR/3429, 1995.

[12] W. Bokhari, L. Rosenson, P. Sphicas. Search for the Top Quark in the Multijet Channel with Double SVX Tagging. CDF/ANAL/TOP/CDFR/3088, 1995.

[13] F. Abe, et al. Evidence for Top Quark Production in $p \bar{p}$ Collisions at $\sqrt{s}=1.8$ TeV. Phys. Rev. D, 50:2966, 1994.

[14] T. Baumann, J. Konigsberg, M. Franklin. A Top Dilepton Analysis Using Hadronic Taus. CDF/ANAL/TOP/CDFR/2325, 1994; M. Gallinaro, M. Hohlmann. Observation of Top Dileptons with Hadronically Decaying Tau Leptons. CDF/ANAL/TOP/CDFR/3461, 1995.

[15] F. Abe, et al. Measurement of the Antiproton-Proton Total Cross Section at $\sqrt{s}=546$ and $1800 \mathrm{GeV}$. Phys. Rev. D, 50:5550, 1994.

[16] Particle Data Group; D.E. Groom, et al. Review of Particle Physics. Eur. Phys. J., C15:1, 2000. Section 34.5.2 contains a discussion of the relationship between rapidity and pseudorapidity.

[17] F. Abe, et al. The CDF Detector: An Overview. Nucl. Inst. Meth. Phys. Res., A271:387, 1988.

[18] D. Amidei, et al. The CDF Silicon Vertex Detector. Nucl. Inst. Meth. Phys. Res., A350:73, 1994.

[19] D. Amidei, et al. SVX': The New CDF Silicon Vertex Detector. Nucl. Inst. Meth. Phys. Res., A360:137, 1995. 
[20] F. Snider, et al. The CDF Vertex Time Projection Chamber System. Nucl. Inst. Meth. Phys. Res., A268:75, 1988.

[21] F. Bedeschi, et al. Design and Construction of the CDF Central Tracking Chamber. Nucl. Inst. Meth. Phys. Res., A268:50, 1988.

[22] L. Balka, et al. The CDF Central Electromagnetic Calorimeter. Nucl. Inst. Meth. Phys. Res., A267:272, 1988.

[23] S. Bertolucci, et al. The CDF Central and Endwall Hadron Calorimeter. Nucl. Inst. Meth. Phys. Res., A267:301, 1988.

[24] G. Ascoli, et al. CDF Central Muon Detector. Nucl. Inst. Meth. Phys. Res., A268:33, 1988 .

[25] G. Foster, et al. A Fast Hardware Track Finder for the CDF Central Tracking Chamber. Nucl. Inst. Meth. Phys. Res., A269:93, 1988.

[26] D. Gerdes. Study of Conversion Removal for the Lepton + Jets Sample. CDF/ANAL/TOP/CDFR/2903, 1995.

[27] CDF Dilepton Working Group. Event Selection for the Top Dilepton Analysis. CDF/DOC/TOP/CDFR/2862, 1995.

[28] M. Kruse, J. Konigsberg. Top Dilepton Analysis - Update to $110 p b^{-1}$. CDF/DOC/TOP/CDFR/3482, 1997.

[29] M. Kruse, J. Konigsberg. Top Dilepton Background from Radiative Z's. CDF/DOC/TOP/CDFR/3672, 1996.

[30] F. Abe, et al. Topology of Three-Jet Events in $p \bar{p}$ Collisions at $\sqrt{s}=1.8 \mathrm{TeV}$. Phys. Rev. D, 45:1448, 1992.

[31] F. Abe, et al. Study of Four-Jet Events and Evidence for Double Parton Interactions in $p \bar{p}$ Collisions at $\sqrt{s}=1.8$ TeV. Phys. Rev. D, 47:4857, 1993. 
[32] The CDF Run Ia datasets that we have used are better known as the "University of Chicago" inclusive lepton sets. Further details are available on the University of Chicago HEP webpage at http://hep.uchicago.edu/cdf/inclu_pad.html. A copy of these datasets is currently accessible on cdfsga in /data79/top/ana/run1a_inclusiveleptons.

[33] CDF Top Analysis Group. Sample Selection for Run 1B Top Search. CDF/ANAL/TOP/CDFR/2966, 1996.

[34] The final Run Ib electron and muon datasets are known as the Topfnd Stripped Inclusive Lepton Samples produced by the Top analysis group at CDF. These were originally located on FNALD in CDF\$TOP4_DATA:[ANA.HPTE] (electrons) and CDF\$TOP3_DATA:[ANA.HPTM] (muons) and are now available on cdfsga in /data79/top/ana/hpte (4643 files) and /data79/top/ana/hptm (4644 files).

[35] We are referring here to the cosmic ray filter written by Phil Schlabach.

[36] M. Kruse. Observation of Top Quark Pair Production in the Dilepton Decay Channel from Proton-Antiproton Collisions at $\sqrt{s}=1.8 \mathrm{TeV}$. CDF/THESIS/TOP/PUBLIC/4632, 1996.

[37] S. Dawson. SUSY and Such. hep-ph/9612229, 1997.

[38] X. Tata. Supersymmetry: Where It Is And How To Find It. hep-ph/9510287, 1995.

[39] M. Ahmed, et al. Search for the Lepton-Family-Number Nonconserving Decay $\mu^{+} \rightarrow e^{+} \gamma$. hep-ex/0111030, 2001; M. L. Brooks, et al. New Limit for the Family-Number Non-conserving Decay $\mu^{+} \rightarrow e^{+} \gamma$. hep-ex/9905013, 1999.

[40] P. Fayet. Phys. Lett., B69:489, 1977. G. Farrar, P. Fayet. Phys. Lett., B76:575, 1978.

[41] H. Goldberg. Phys. Rev. Lett., 50:1419, 1983. J. Ellis, J. Hagelin, D.V. Nanopoulos, K. Olive, and M. Srednicki. Nucl. Phys., B238:453, 1984. 
[42] W. Beenakker, R. Höpker, M. Spira, P. M. Zerwas. Gluino-Pair Production at the Tevatron. hep-ph/9505416, 1995.

[43] W. Beenakker, R. Höpker, M. Spira, P. M. Zerwas. Squark and Gluino Production at Hadron Colliders. hep-ph/9610490, 1996.

[44] W. Beenakker, R. Höpker, M. Spira. PROSPINO A Program for the Production of Supersymmetric Particles in Next-to-Leading Order QCD. hep-ph/9611232, 1996.

[45] H.L. Lai, J. Botts, J. Huston, J.G. Morfn, J.F. Owen, J.W. Qiu, W.K. Tung, H. Weerts. Global QCD Analysis and the CTEQ Parton Distributions. Phys. Rev. $D, \mathbf{5 1 : 4 7 6 3 , 1 9 9 5 .}$

[46] M. Gluck, E. Reya, A. Vogt. Dynamical Parton Distributions of the Proton and Small-X Physics. Z. Phys. C, 67: 433, 1995.

[47] A.D. Martin, W.J. Stirling, R.G. Roberts. Parton Distributions Updated. Phys. Lett., B306:145, 1993. Erratum-ibid. B309:492, 1993.

[48] S. Behrends. Jet Results From the 1989 Data: New Energy Scale Definition. CDF/ANAL/JET/CDFR/2226, 1993.

[49] S. Behrends. The Ratio of Low $E_{T}$ Jet Cross-Sections at $\sqrt{s}=546$ and 1800 GeV. CDF/ANAL/JET/CDFR/2151, 1993.

[50] P. Maas. Energy Outside a Clustering Cone Radius of 0.4 . CDF/DOC/JET/CDFR/1867, 1992.

[51] A. Caner, L. Galtieri, J. Lys, W. Yao, A. Yagil. Jet Corrections For Top Mass Fitting. CDF/ANAL/TOP/CDFR/2469, 1994.

[52] A. Caner, A. Yagil. Study of Jet E-Scale Using Photon-Jet Balancing. CDF/ANAL/TOP/CDFR/2460, 1994.

[53] S. Kuhlmann. The 10\% Solution. CDF/PHYS/TOP/CDFR/2506, 1994. 
[54] F. Abe, et al. A Two Level Fastbus Based Trigger System for CDF. Nucl. Inst. Meth. Phys. Res., A269:51, 1988.

[55] F. Abe, et al. Measurement of $\sigma \dot{B}(W \rightarrow e \nu)$ and $\sigma \dot{B}\left(Z^{0} \rightarrow e^{+} e^{-}\right)$in $p \bar{p}$ collisions at $\sqrt{s}=1.8 \mathrm{TeV}$. CDF/PUB/ELECTROWEAK/PUB/3312, hep-ex/9509010, 1995.

[56] D. Cronin-Hennessy, A. Beretvas, P.F. Derwent. Luminosity Monitoring and Measurement at CDF. CDF/PUB/ELECTROWEAK/PUB/4956, 1999.

[57] The PYTHIA tz sample was created by Marcus Hohlman at the University of Chicago.

[58] The HERWIG $t \bar{t}$ sample was created by Carlo Grosso-Pilcher at the University of Chicago.

[59] F. Abe, et al. Observation of Top Quark Production in $\bar{p} p$ Collisions with the Collider Detector at Fermilab. Phys. Rev. Lett., 74:2626, 1995.

[60] T. Chikamatsu, et al. Top Dilepton Analysis - Run 1. CDF/ANAL/TOP/CDFR/2851, 1995.

[61] F. Abe, et al. Measurement of $B^{0} \bar{B}^{0}$ Mixing at the Fermilab Tevatron Collider. Phys. Rev. Lett., 67:3351, 1991.

[62] S. Lammel. SUSY Monte Carlo Generation and Simulation for Run 1a. CDF/ANAL/EXOTIC/PUBLIC/2889, 1995.

[63] H. Mitsushio, I. Nakano, Y. Seiya. Measurement of Time Integrated $B^{0} \bar{B}^{0}$ Mixing in Run-Ia $e \mu$ Events. CDF/ANAL/BOTTOM/CDFR/2915, 1995. Supplement. CDF/ANAL/BOTTOM/CDFR/3042, 1995.

[64] S. Drell and T.-M. Yan. Massive Lepton-Pair Production in Hadron-Hadron Collisions at High Energies. Phys. Rev. Lett., 25:316, 1970.

[65] K. Hagiwara, J. Woodside, D. Zeppenfeld. Measuring the WWZ coupling at the Fermilab Tevatron. Phys. Rev. D., 41:2113, 1990. 
[66] J. Ohnemus. Order- $\alpha_{s}$ calculation of hadronic $W^{-} W^{+}$production. Phys. Rev. D., 44:1403, 1991.

[67] J. Ohnemus. Order- $\alpha_{s}$ calculation of hadronic $W^{ \pm} Z$ production. Phys. Rev. D., 44:3477, 1991.

[68] J. Ohnemus and J.F. Owens. Order- $\alpha_{s}$ calculation of hadronic $Z Z$ production. Phys. Rev. D., 43:3626, 1991.

[69] J. Romano and M. Contreras. Background due to Hadron Misidentification in the Top Dilepton Search. CDF/ANAL/TOP/CDFR/2107, 1993.

[70] D. Bortoletto, et al. Top Dilepton Analysis - June 1995 Update. CDF/ANAL/TOP/CDFR/3203, 1995.

[71] O. Helene. Nucl. Inst. Meth. Phys. Res., A212:319, 1983.

[72] J. Conway. Setting Limits and Making Discoveries in CDF. CDF/EXOTIC/PUB/PUBLIC/5236, 2000.

[73] D. Bortoletto. The CDF SVX II Upgrade for the Tevatron Run II. VERTEX96, 5th International Workshop on Vertex Detectors, Chia, Italy, June 16-21, 1996. CDF/PUB/TRACKING/PUBLIC/4015, 1997.

[74] S. Eno. The D0 and CDF Run II Detectors. Fermilab Run II Higgs and SUSY Workshop, Batavia, Illinois, May 14, 1998.

[75] M. Coca, et al. Proposal for the Summer 2002 Top Dilepton Analysis and Top Dilepton Dataset. CDF/PHYS/TOP/5676, 2001.

[76] H. Frisch. Separating Top and Z's in Dilepton Events inside the Z Mass Window. CDF/TOP/ANAL/3387, 1995. 


\section{Vita}

Chadd Smith

and graduated valedictorian

in 1990. He subsequently enrolled at Virginia Tech in Blacksburg, Virginia, where he dual majored in Physics and Mathematics. In 1992, he participated in a summer research program through the University of Tennessee and Oak Ridge National Laboratory, where he studied the optical properties of thin films of frozen hydrocarbons in the laboratory of Dr. Ed Arakawa and Samuel McKenzie. For the following two years, he worked with Dr. Leo Piilonen of Virginia Tech on the MEGA $(\mu \rightarrow e \gamma)$ experiment at Los Alamos Meson Physics Facility and spent two summers at LAMPF in New Mexico. In May 1994, he received his Bachelor of Science degree in Physics, with a subspecialty in observational astronomy. Later that fall, he entered the Ph.D. program at Johns Hopkins University and came under the advisement of Dr. Bruce Barnett in the experimental high energy physics group. He joined the CDF collaboration after working at Fermilab during the summer of 1995. For the next few years, he was involved in the extensive testing of silicon microstrip detectors for the SVX II detector and managed a small group of undergraduate physics students. He also served as a teaching assistant for the introductory physics and undergraduate quantum mechanics courses, and in 1997 he received the Outstanding Teaching Assistant Award from the Department of Physics and the American Association of Physics Teachers (AAPT). In his spare time, he also taught MCAT physics test preparation courses for The Princeton Review and tutored undergraduate physics students. In June 2000, his wife, Dr. Pai C. Meng, began her med/peds residency at University of Chicago Hospitals. He continued his thesis research at Fermilab until receiving his Ph.D. in

Physics in January 2002. He and his wife currently reside in the neighborhood of Hyde Park along with several cacti, bonsai, herbs, and a bowl of Sea Monkeys. 
